\title{
REPRESENTATION OF EDDIES IN CLIMATE MODELS BY A POTENTIAL VORTICITY FLUX
}

by

\author{
Richard M. Wardle \\ B.Sc. University of York, England \\ (1993) \\ Submitted in partial fulfillment of the \\ requirements for the degree of \\ Doctor of Philosophy \\ at the
MASSACHUSETTS INSTITUTE OF TECHNOLOGY
and the \\ WOODS HOLE OCEANOGRAPHIC INSTITUTION
}

February 1999

(C) Richard M. Wardle 1999

The author hereby grants to MIT and to WHOI permission to reproduce and to distribute copies of this thesis document in whole or in part.

Signature of Author

Joint Program in Physical Oceanography Massachusetts Institute of Technology

Woods Hole Oceanographic Institution February, 1999

Certified by $\ldots \ldots \ldots$

John Marshall

Professor of Physical Oceanography

Thesis Supervisor

Accepted by

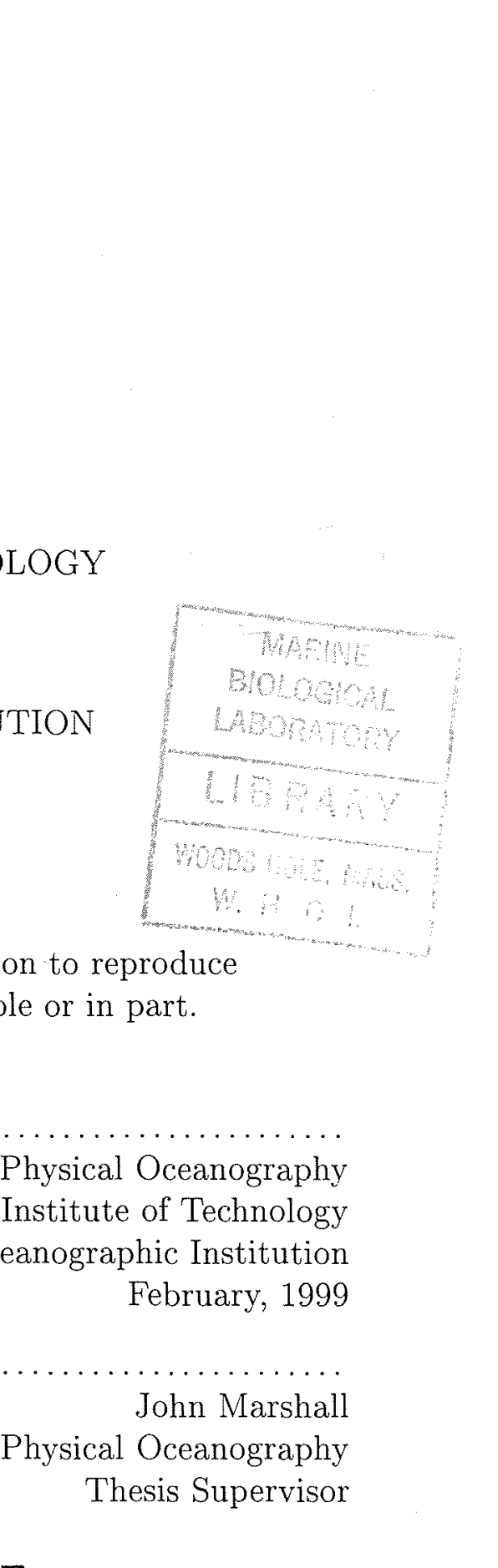

W. Brechner Owens

Chairman, Joint Committee for Physical Oceanography Massachusetts Institute of Technology

Woods Hole Oceanographic Institution 


\title{
REPRESENTATION OF EDDIES IN CLIMATE MODELS BY A POTENTIAL VORTICITY FLUX
}

\author{
by \\ Richard M. Wardle
}

\begin{abstract}
Submitted in partial fulfillment of the requirements for the degree of Doctor of Philosophy at the Massachusetts Institute of Technology and the Woods Hole Oceanographic Institution
\end{abstract}

February, 1999

\begin{abstract}
This thesis addresses the parameterization of the heat and momentum transporting properties of eddy motions for use in three-dimensional, primitive equation, z-coordinate atmosphere and ocean models. Determining the transport characteristics of these eddies is fundamental to understanding their effect on the large-scale ocean circulation and global climate.

The approach is to transform the primitive equations to yield the altered 'transformed Eulerian mean' (TEM) equations. The assumption is made that the eddy motions obey quasigeostrophic dynamics while the mean flow obeys the primitive equations. With this assumption, the TEM framework leads to the eddies appearing as one term, which acts as a body force in the momentum equations. This force manifests itself as a flux of potential vorticity (PV) - a quantity that incorporates both eddy momentum and heat transporting properties. Moreover, the dynamic velocities are those of the residual mean circulation, a much more relevant velocity for understanding heat and tracer transport.

Closure for the eddy PV flux is achieved through a flux-gradient relationship, which directs the flux down the large scale PV gradient. For zonal flows, care is taken to ensure that the resulting force does not generate any net momentum, acting only to redistribute it. Neglect of relative vorticity fluxes in the PV flux yields the parameterization scheme of Gent and McWilliams.

The approach is investigated by comparing a zonally-averaged parameterized model with a three dimensional eddy-resolving calculation of flow in a stress-driven channel. The stress at the upper surface is communicated down the water column to the bottom by eddy form drag. Moreover, lateral eddy momentum fluxes act to strengthen and sharpen the mean flow, transporting eastward momentum up its large scale gradient. Both the vertical momentum transfer and lateral, upgradient momentum transfer by eddies, are captured in the parameterized model.

The advantages of this approach are demonstrated in two further zonal cases: 1) the spin-down of a baroclinic zone, and 2) the atmospheric jet stream.

The time mean TEM approach and the eddy PV flux closure are explored in the context of an eddy-resolving closed basin flow which breaks the zonal symmetry.
\end{abstract}


Decomposition of eddy PV fluxes into components associated with advective and dissipative effects suggest that the component associated with eddy flux divergence, and therefore forcing of the mean flow, is mainly directed down the large scale gradient and can be parameterized as before. Thus, the approach can be used to capture eddy transport properties for both zonal mean and time mean flows.

The PV flux embodies both the eddy heat and momentum fluxes and so presents a more unified picture of their transferring properties. It therefore provides a powerful conceptual and practical framework for representing eddies in numerical models of the atmsophere and ocean.

Thesis Supervisor: John Marshall, Professor,

Program in Atmospheres, Oceans, and Climate Massachusetts Institute of Technology 


\section{Acknowledgements}

The work in this thesis was funded by grants from NSF, (OCE-9634331, OCE9503895), ONR (N00014-95-1-0967), and by a fellowship from the Joint Program on the Science and Policy of Global Change at MIT. This support is gratefully acknowledged.

I would like to thank Joe Pedlosky for his patience and steady guidance in the first year of graduate school, and for many words of advice thereafter. Joe taught me to be more confident, to explain things clearly, and "unless something exceptional happens, you never throw to first base from the outfield!"

My supervisor, John Marshall discussed many research ideas with me and after we settled on a thesis topic, he got me started in the right direction. Throughout the thesis John has never restrained himself from sharing his unabashed opinions of my work, me, and many other topics. His initiative and enterprise provided me with all the computer resources necessary to perform many, many model runs, most of which do not appear in the thesis.

Alan Plumb acted as my de facto advisor, providing the necessary scientific guidance, critical advice, and feedback that I needed to complete the thesis. Alan's knowledge of the TEM and eddy characteristics are unparalleled, and I took away as much of this as possible, each time that we spoke. For this guidance, I am truly grateful.

Every other member of my thesis committee contributed positively and deserve credit. Mike Spall's modeling experience and questions about fluxes and their divergences were extremely beneficial. Ray Schmitt always seemed pleased to hear from me, and was genuinely interested in the salinity-free work. Jack Whitehead always gave me productive feedback on my written work and asked me questions that I had not thought about. Finally, I thank Glenn Flierl for always being available to answer trivial scientific queries and for acting as Chair of the defence.

I have been extremely fortunate to use a numerical model in continual development on machines that constantly change. I was fortunate because I had the patient help and understanding of the model developers - Chris Hill and Alistair Adcroft. Chris was abs(abs(absolutely)) willing to suffer my code additions and disregard my feedback. Alistair kept me laughing in the final weeks of writing, by jokingly informing me daily of a bug in the code that invalidated my results. Oh, how I did laugh.

I met a few people who made bureaucracy a lot easier to take. Ronni Schwartz spiced up my trips to the ninth floor and gave me almost free reign over the JP car. Abbie Alvin in the education office made me feel very welcome when I first arrived. First Jane $\mathrm{McNabb}$, and then Lisa Mcfarren, proved to be the pleasing and useful secretaries that I needed.

The pleasure of being in the Joint Program is due in no small part to the members of the community in which I found myself. During classes I enjoyed antagonizing my classmates Melissa, Sandra, Dan, Jubao, and the two Mishas, while simultaneously picking 
up scraps of their collective abilities. Jubao has been a close friend and officemate, and allowed me to be uncle to his son. Tom Haine allowed me to crush him at squash, out score him at footy, and out drink him at the Druid. I never out drank Jim Gunson, but enjoyed trying to keep pace. During my time as PO alpha male I've received much satisfaction from the worship of Joe LaCasce, Francois Primeau, Alex Ganachaud, Rebecca Morss, Stephanie Harrington, Lous St. Laurent, and Juan Botella. My main disciple Brian Arbic, helped to clarify the thesis by diligently reading through the penultimate draft. Mick Follows was the ideal roommate at conferences and confidant down the hall. When my data overflowed, Linda Meinke was always understanding and willing to help. Jochem "what's new here?" Marotzke and Carl "does it make a difference?" Wunsch always provided me with useful feedback during and after my seminars, and Paola Rizzoli was always willing to talk.

The various denizens of 1419 have made the day at the office interesting and enjoyable. The golden age of the monarchy was particularly fun. Keith Alverson was always willing to talk, argue, and bet, but unfortunately never willing to pay up. Chris Edwards loved listening to scintillating stories and arousing our curiosity with unabated caginess. Finally, Natalia Beliakova, after becoming one of the guys, was a tremendous source of fun and friendship. There is hope of a 1419 revival with Jake Gebbie.

I have also been fortunate to have a desk in Woods Hole much to the chagrin of Lyn Harris and Albert Fischer, my favourite WHOI officemates.

I've had many friends that have nothing to do directly with my studies at MIT, but have provided useful distractions and amusement - Thanks. In particular, Simon McClusky played soccer with me for five years and joined me in commentating on numerous Patriots, Red Sox, Bulls, and World Cup games. The best thing about Simon is his wife, Sonja Glasson, who keeps Simon in check and is always fun to be around. The Los Guapos football club have allowed me to strut my stuff for most Sundays in the last two years and have given me many opportunities to socialize.

In the four years since I met Dacia she has been a constant source of love and companionship, which has benefited me without measure. Equally supportive and caring have been Dacia's family who have always welcomed me into their household, and made me feel that there is always a place to turn. Thank you.

In the final year of the thesis, I wooed Heather with talk of eddies and of cream making swirls in coffee. Since then she has stuck with me and has been a tremendous source of fun, motivation and love.

I am also grateful to my Mum and Dad, who have always supported me from afar even though I didn't have a proper job. Thanks also goes to my older siblings Angela and Glenn who have both given me advice and encouragement, and have amused me with questions of fish farms and of sunken ships. 


\section{Contents}

$\begin{array}{ll}\text { Abstract } & 3\end{array}$

$\begin{array}{ll}\text { Acknowledgements } & 5\end{array}$

1 Introduction $\quad 16$

1.1 Eddy variability in the ocean . . . . . . . . . . . . . . 18

1.2 Prior representation of eddy transfer . . . . . . . . . . . . . . 25

1.2.1 Atmospheric eddies . . . . . . . . . . . . . . . 25

1.2 .2 Ocean eddies . . . . . . . . . . . . . . . . . . 28

1.3 Focus and overview of the thesis . . . . . . . . . . . . . . . . 32

2 The Dynamical Framework $\quad 35$

2.1 Introduction . . . . . . . . . . . . . . . . . . . . . . . . . . . . . . . .

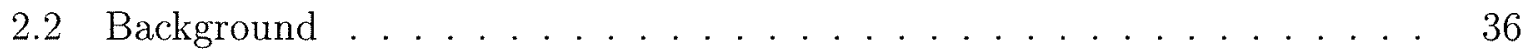

2.2.1 Zonal mean coordinate systems . . . . . . . . . . . . . 36

2.2.2 Quasigeostrophic PV, PV sheets, and eddy PV fluxes . . . . . . 37

2.3 The Eulerian mean . . . . . . . . . . . . . . . . . . 43

2.4 The transformed Eulerian mean (TEM) . . . . . . . . . . . . . . . 45

2.5 The TEM in the limit of quasigeostrophic eddies . . . . . . . . . . . 47

2.5.1 Scaling analysis . . . . . . . . . . . . . . . 47

2.6 Representation of eddies by a PV flux . . . . . . . . . . . . . . . . 53

2.7 The equations for time mean flows . . . . . . . . . . . . . . . . . . . . . . . . . . . . 56

2.8 The equations for zonal mean flows . . . . . . . . . . . . . . . . . 57

2.8.1 Eddy-propagation, transport and integral constraints . . . . . 58

2.8.2 The limit of vanishing relative vorticity flux . . . . . . . . . 62

2.9 Summary . . . . . . . . . . . . . . . . . . 6 64

3 Closure for the eddy PV flux $\quad 66$

3.1 Introduction . . . . . . . . . . . . . . . . . 66

3.2 Downgradient PV transfer . . . . . . . . . . . . . . . 66

3.3 Specification of the $K$ 's for zonal mean flows . . . . . . . . . . . . . . . . 69 
4 Zonal Mean Flows $\quad 72$

4.1 Introduction . . . . . . . . . . . . . . . . . 72

4.2 Eastward Flow in a $\beta$-plane channel . . . . . . . . . . . . . . . . . 73

4.2.1 The eddy-resolving model . . . . . . . . . . . . . . . 73

4.2.2 The parameterized model . . . . . . . . . . . . . . . 91

4.3 Spin-down of a baroclinic zone on a $\beta$-plane . . . . . . . . . . . . 98

4.3.1 The eddy-resolving model . . . . . . . . . . . . . . . . . 98

4.3.2 The parameterized model . . . . . . . . . . . . . . . 104

4.4 Tropospheric eddies in the atmosphere . . . . . . . . . . . . . 108

4.5 Summary . . . . . . . . . . . . . . . . . 119

5 Time Mean Flows $\quad 121$

5.1 Introduction . . . . . . . . . . . . . . . . . . . . . . . . . . . . . . . 121

5.2 Flow in a double-gyre ocean . . . . . . . . . . . . . . . . . . . . . . . . . . . . . . . . . . . 122

5.2 .1 Model setup . . . . . . . . . . . . . . . 123

5.2.2 Flow evolution and equilibration . . . . . . . . . . . . 127

5.2 .3 Eddy transfer statistics . . . . . . . . . . . . . . . . . 136

5.2.4 Decomposition of the eddy flux of PV . . . . . . . . . . . . 140

5.3 Representation of the time mean eddy-forcing . . . . . . . . . . . . 151

5.4 Summary . . . . . . . . . . . . . . . . . . 155

6 Summary and Conclusions $\quad 156$

6.1 Summary of the thesis . . . . . . . . . . . . . . . . 156

6.2 Future Work . . . . . . . . . . . . . . . . 161

$\begin{array}{ll}\text { A Model details } & \mathbf{1 6 3}\end{array}$

A.1 Model equations . . . . . . . . . . . . . . . . . . . . 163

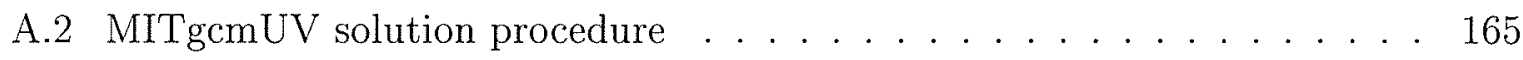

A.3 Boundary conditions . . . . . . . . . . . . . . . . . . 168

$\begin{array}{ll}\text { References } & 170\end{array}$ 


\section{List of Figures}

1.1 A schematic picture of the 'wedge of instability' description of baroclinic instability for the troposphere. . . . . . . . . . . . . . . 21

1.2 The Eady growth rate calculated from Levitus (1994) annual climatology 23

1.3 TOPEX/POSEIDON rms sea-surface height variability averaged over 4 years . . . . . . . . . . . . . . . . . . . 24

2.1 Schematic picture of lateral boundary PV sheets. . . . . . . . . . 40

2.2 Schematic meridional profiles of the Eliassen-Palm flux (arrows) and its divergence (solid and dashed lines) for the most unstable (a) Eady and (b) Charney modes . . . . . . . . . . . . . . . . . .

2.3 Schematic diagram of the procedure followed in chapter2. The two steps shown yield a set of governing equations for the transformed Eulerian mean flow in which the eddy term appears a PV flux acting as a body force in the momentum equations. The prognostic and advective velocity is that of the residual mean circulation. . . . . . . . . . . . . .

4.1 A schematic diagram of the channel model domain . . . . . . . . .

4.2 Surface velocities from the eddy-resolving model after 420, 460, and 3900 days. The temperature is contoured and shaded with lighter shading denoting warmer lighter water. The panels on the right display the corresponding mean zonal surface velocity in $m s^{-1} \ldots \ldots \ldots \ldots$

4.3 The times series of the available potential energy (heavy line) and kinetic energy (thin line) per unit mass per unit volume. The available potential and kinetic energies increase until about day 400, when the ratio APE:KE is $7.5: 1$ at which point Rossby wave breaking occurs, releasing available potential energy and causing a conspicuous decrease in the available potential and kinetic energies. After six years or so, a statistically steady state is reached where available potential energy is three times the kinetic energy. . . . . . . . . . . . . . . . . 
4.4 The eddy-resolving eastward flow in a $\beta$-plane channel. The time-averaged meridional cross-sections of: (a) zonal mean zonal velocity $\left(\mathrm{ms}^{-1}\right)$; (b) zonal mean temperature. The time average was taken from 10 to 20 years. 79

4.5 The wind-driven Eulerian mean streamfunction, $\chi_{E u l}$, in (a) is almost exactly canceled by $\chi_{\text {flux }}$ in (b). Units are Sv. The result is the near vanishing of the residual mean overturning circulation. . . . . . . . .

4.6 The depth integrated PV flux. At any latitude the bottom drag does not exactly balance the surface stress and the difference is balanced by an eddy flux of PV. A positive (eastward) body force is exerted on the zonal flow in the center of the channel and a negative (westward) body force is exerted on the flanks of the jet. . . . . . . . . . . . . . .

4.7 The depth average of the zonal mean zonal velocity in the eddy-resolving equilibrated state. . . . . . . . . . . . . . . .

4.8 Eddy momentum fluxes associated with a 'Banana-shaped' eddy. The eddy velocities $\left(u^{\prime}, v^{\prime}\right)$ have a zero zonal mean, but their product can be nonzero if the eddy, as here, is anisotropic. To the south of the jet axis the trough slopes in a southwest-northeast sense inducing a northward eddy flux of eastward eddy zonal velocity $\overline{u^{\prime} v^{\prime}}>0$. North of the jet axis, the troughs tilt southeast-northwest and $\overline{u^{\prime} v^{\prime}}<0$. Thus the effect of the eddies is to transfer eastward momentum into the center of the eastward jet from the flanks. This effect is well known in the atmospheric literature, see for example, Starr (1968) and Houghton (1977). . . . . . . . . . .

4.9 (a). Surface velocities from the eddy-resolving model after 3660 days. The temperature is contoured and shaded with lighter shading denoting warmer lighter water; (b) Surface map of $\mathbf{v}^{\prime} q^{\prime} \cdot \nabla \bar{q}^{x}$ after 3660 days. Positive (negative) values are contoured with a solid (broken) line. The contour interval is $5 \times 10^{-13} \mathrm{~s}^{-3}$. (c) $\overline{\mathrm{v}^{\prime} q^{x}} \cdot \nabla \bar{q}^{x}$ at this time. (d) time average $\overline{\mathbf{v}^{\prime} q^{x t}} \cdot \nabla \bar{q}^{x t}$. The negative values indicate downgradient transfer of quasigeostrophic PV. 85

4.10 Meridional cross-sections of; (a) The eddy PV flux $\overline{v^{\prime} q^{x t}}$ is dominated by the boundary sheets with divergence at depth and surface convergence; (b) The eddy-flux of temperature, ${\overline{v^{\prime} T^{\prime}}}^{x t}$. The contour interval is $1 \times 10^{-3}$ $m s^{-1} K$. (c) The eddy-flux of momentum, $\overline{u^{\prime} v^{x}}$. The contour interval is $1 \times 10^{-3} \mathrm{~m}^{2} \mathrm{~s}^{-2} . \ldots \ldots \ldots \ldots \ldots \ldots \ldots \ldots \ldots$

4.11 The diagnosed transfer coefficients for quasigeostrophic potential vorticity in the upper PV sheet (circles) and the lower PV sheet (crosses) in the statistically steady state. . . . . . . . . . . . . . . . . .

4.12 Plot of $\overline{\left(v^{\prime} q^{\prime}\right)}$ vs. $\bar{q}_{y}$ for the PV sheets. . . . . . . . . . . . 89 
4.13 The depth-integrated Eliassen-Palm flux divergence calculated using the geostrophic streamfunction (solid line), as described in the Appendix, and from the full fields (dashed line). The very close correspondence between the two demonstrates the assumption that the eddies are quasigeostrophic in nature is an excellent for the stress-driven channel flow.

4.14 (a) The time series of the global average $K\left(m^{2} s^{-1}\right)$ in the parameterized model; (b) The steady state $K$ profile with $\kappa_{\text {ref }}=1050 m^{s} s^{-1}(\gamma=-3.21) .92$

4.15 Steady state meridional cross-sections from the parameterized model: (a) zonal mean zonal velocity $\left(m s^{-1}\right)$; (b) zonal mean temperature. . . . . .

4.16 The stress (dashed) and eddy flux of quasigeostrophic potential vorticity (solid) terms in the steady state momentum equation for; (a) the upper layer; (b) the interior; (c) the lower layer. Equation 4.13 is exactly satisfied. 95

4.17 (a) The PV flux $\left(\overline{v^{\prime} q^{\prime}}\right)$ is concentrated at the boundaries; (b) The depth integrated PV flux. Its effect is to exert a positive (eastward) body force on the zonal momentum in the center of the channel, and a negative (westward) body force in the flanks of the jet, consistent with the eddy characteristics diagnosed from the eddy-resolving model. . . . . . . . . . . .

4.18 The depth average of the zonal mean zonal velocity in the (a) parameterized model; (b) eddy-resolving model. . . . . . . . . . . . . . 97

4.19 Spin-down of a baroclinic zone. The initial temperature cross-section. . . 98

4.20 Spin-down of a baroclinic zone. The times series of the available potential energy and kinetic energy per unit mass per unit volume. . . . . . . .

4.21 Spin-down of a baroclinic zone. Surface temperature and velocities from the eddy-resolving channel model after 165, 180, 240 and 3600 days. The temperature is contoured and shaded with lighter shading denoting warmer water. . . . . . . . . . . . . . . . .

4.22 Spin-down of a baroclinic zone. Zonal average fields from the eddy resolving model. The time-averaged meridional cross-sections of: (a) zonal mean zonal velocity $\left(\mathrm{ms}^{-1}\right)$; (b) zonal mean temperature; (c) zonal mean surface velocity $\left(\mathrm{ms}^{-1}\right)$. The time average was taken over the last three years of integration. . . . . . . . . . . . . .

4.23 Spin-down of a baroclinic zone. Zonal average fields from the parameterized model. The final-state meridional cross-sections of: (a) zonal mean zonal velocity $\left(\mathrm{ms}^{-1}\right)$; (b) zonal mean temperature; (c) zonal mean surface velocity $\left(m s^{-1}\right) \ldots \ldots \ldots \ldots \ldots \ldots$

4.24 Spin-down of a baroclinic zone. Zonal average fields from the parameterized model when the relative vorticity fluxes are ignored. The final-state meridional cross-sections of: (a) zonal mean zonal velocity $\left(\mathrm{ms}^{-1}\right)$; (b) zonal mean temperature; (c) zonal mean surface velocity $\left(\mathrm{ms}^{-1}\right) \ldots \ldots$. 
4.25 Initial meridional cross-sections for the troposphere experiments; (a) potential temperature; (b) $\bar{\theta}_{e q}$, the relaxation potential temperature; (c) $\tau$, the relaxation timescale in days. . . . . . . . . . . . . . .

4.26 The meridional cross-sections after 1000 days for the experiment with no eddy-forcing: (a) potential temperature; (b) zonal velocity; (c) residual mean overturning streamfunction; (d) $950 \mathrm{mb}$ winds. . . . . . . . . .

4.27 The meridional cross-sections after 1000 days for the experiment with eddy-forcing: (a) potential temperature; (b) zonal velocity; (c) residual mean overturning streamfunction; (d) $950 \mathrm{mb}$ winds. . . . . . . . . . 114

4.28 (a). The Eliassen-Palm flux divergence; (b) The column integrated EliassenPalm flux divergence. The eddies exert a westerly force at mid-latitudes and easterly forces in the tropics and toward the poles. . . . . . . . .

4.29 The meridional profiles after 1000 days for the experiment with the limiting case of the eddy-forcing: (a) potential temperature; (b) zonal velocity; (c) residual mean overturning streamfunction; (d) $950 \mathrm{mb}$ winds. . . . . . . .

4.30 The meridional cross-sections of; (a) the Eliassen-Palm flux divergence; (b) the column integrated Eliassen-Palm flux divergence. The columnintegrated divergence is zero because relative vorticity fluxes have been ignored. There is no lateral momentum flux $\left(E_{y}=0\right)$ and so there is only vertical transfer of momentum, due to the lateral eddy buoyancy fluxes. .

5.1 A schematic diagram of the double-gyre model domain. A sinusoidal wind stress drives subtropical and subpolar gyres to the south and north of the zero wind stress curl line. . . . . . . . . . . . . .

5.2 Instantaneous surface temperature and velocity fields for the double-gyre experiment at (a) 0.5 years, (b) 1 year, (c) 3 years, (d) 6 years, (e) 10 years, (f) 15 years, (g) 18 years, (h) 25 years. The temperature field is contoured with warmer water denoted by lighter shading. Velocities are denoted by arrows. . . . . . . . . . . . . . . . . . . . . energy (thin line) per unit mass per unit volume. . . . . . . . . . . . 130

5.4 Time mean surface velocity and temperature fields . . . . . . . . . . . 131

5.5 Fields of; (a) The time mean barotropic streamfunction $\bar{\psi}$. The range plotted is $\left(-40 \times 10^{6} \leq \bar{\psi} \leq 40 \times 10^{6}\right)$. The contour interval is $4 \times 10^{6}$. Units are $m^{3} s^{-1}$. (b) The time mean surface (depth $\left.=-50 \mathrm{~m}\right) \mathrm{PV} \bar{q}$. The range plotted is $\left(-3 \times 10^{-5} \leq \bar{q} \leq 2 \times 10^{-4}\right)$. The contour interval is $1 \times 10^{-5}$. Units are $s^{-2} \ldots \ldots \ldots \ldots \ldots \ldots$ 
5.6 Plots of the mean PV, $\bar{q}$, at each level of the model. The range plotted is $\left(-3 \times 10^{-5} \leq \bar{q} \leq 2 \times 10^{-4}\right)$. The contour interval is $1 \times 10^{-5}$. Units are $s^{-2}$. The PV sheet is evident in the upper level of the model. Weak PV gradients are present at every other level in the model. PV gradients in these levels are typically an order of magnitude smaller than the planetary vorticity gradient $\beta . \ldots \ldots \ldots \ldots \ldots \ldots \ldots$

5.7 Time mean surface eddy kinetic energy field . . . . . . . . . . . 136

5.8 Time mean field of the barotropic residual mean streamfunction The range plotted is $\left(-40 \times 10^{6} \leq \bar{\psi} \leq 40 \times 10^{6}\right)$. The contour interval is $4 \times 10^{6}$. Units are $m^{3} s^{-1}$.

5.9 The eddy PV flux $\overline{\left(\mathbf{u}^{\prime} q^{\prime}\right)}$ superposed with the mean PV field $\bar{q}$. Every other vector is shown. The $\operatorname{sign}\left(\overline{\left(\mathbf{u}^{\prime} q^{\prime}\right)} \cdot \nabla \bar{q}\right)$ is shaded. Gray indicates regions of downgradient PV transfer with $\overline{\left(\mathbf{u}^{\prime} q^{\prime}\right)} \cdot \nabla \bar{q}<0$. White indicates $\overline{\left(\mathbf{u}^{\prime} q^{\prime}\right)} \cdot \nabla \bar{q}>0$ or upgradient PV transfer. . . . . . . . . . .

5.10 Time mean surface field of eddy enstrophy $e$. The contour interval is $2.5 \times 10^{-10} \mathrm{~s}^{-2}$

5.11 Time mean surface field of the sign $(\nabla \bar{q} \cdot \nabla \bar{\psi})$ and Eulerian mean barotropic streamfunction. Regions shaded in gray indicate $\nabla \bar{q} \cdot \nabla \bar{\psi}<0$. Regions

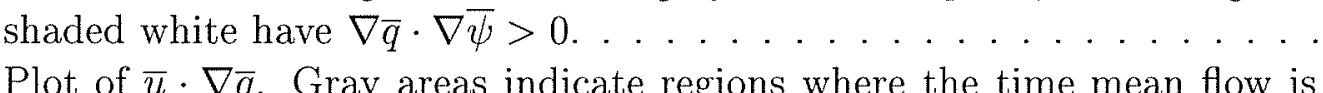

5.12 Plot of $\bar{u} \cdot \nabla \bar{q}$. Gray areas indicate regions where the time mean flow is nearly conservative, $\bar{u} \cdot \nabla \bar{q} \approx 0$. The close conservation of $\bar{q}$ in the jet region enables rationalization of the eddy PV flux components that are associated with advective and dissipative effects. The eddy flux component balancing mean flow advection is non-divergent and does not force the mean flow. The eddy flux component that contains all of the divergent flux is directed down the mean $\bar{q}$ gradient and plays the role of balancing the dissipation of eddy enstrophy White areas indicate regions of non-conservation of $\bar{q}$ where the mean flow is driven across $\bar{q}$ contours by the wind stress curl. .

5.13 The unit vectors $\mathbf{n}$ and $\mathbf{s . ~} \mathbf{n}=\frac{\nabla \bar{q}}{|\nabla \bar{q}|}$ is directed along the mean PV gradient. $\mathbf{s}=\mathbf{k} \times \mathbf{n}$ is directed in the upstream direction. Adapted from Plumb (1986).145

5.14 The eddy PV flux $\overline{\left(\mathbf{u}^{\prime} q^{\prime}\right)}$ Rot superposed with the contours of eddy enstrophy, e. Every other vector is shown. . . . . . . . . . . . . . 146

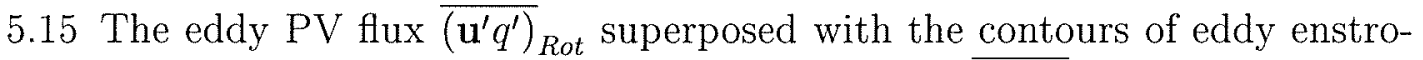
phy, e. Every other vector is shown. The sign $\left({\overline{\left(\mathbf{u}^{\prime} q^{\prime}\right)_{D i v}}} \cdot \nabla \bar{q}\right)$ is shaded. Gray indicates regions of ${\overline{\left(\mathbf{u}^{\prime} q^{\prime}\right)}}_{D i v} \cdot \nabla \bar{q}<0$. White indicates ${\overline{\left(\mathbf{u}^{\prime} q^{\prime}\right)}}_{D i v} \cdot \nabla \bar{q}>0.147$ 
5.16 (a) The $\operatorname{sign}\left(\overline{\left(\mathbf{u}^{\prime} q^{\prime}\right)} \cdot \nabla \bar{q}\right)$. (b) The $\operatorname{sign}\left({\overline{\left(\mathbf{u}^{\prime} q^{\prime}\right)}}_{D i v} \cdot \nabla \bar{q}\right)$. Areas shaded in gray are regions where the quantity is negative indicating downgradient PV transfer. Subdomain A is indicated. Within the subdomain, white areas correspond to to regions of upgradient PV transfer. However, as figure 5.17 shows, the white areas in (b) correspond to regions where the upgradient flux in vanishingly small and thus have little effect on the mean flow. . . . . . . . . . . . . . . . . . . . . . . . . 148

5.17 The upper level eddy fluxes; (a) $\overline{\left(\mathbf{u}^{\prime} q^{\prime}\right)}$ superposed on the $\operatorname{sign}\left(\overline{\left(\mathbf{u}^{\prime} q^{\prime}\right)} \cdot \nabla \bar{q}\right)$.

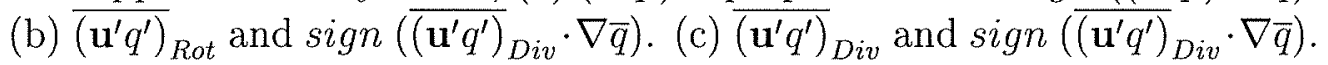
Shading as before. . . . . . . . . . . . . . . . . . . . . 149

5.18 Scatter diagram for mean PV $\bar{q}$ against mean streamfunction $\bar{\psi}$ for the region $0 \leq x \leq 1000 \mathrm{~km}, 500 \leq y \leq 1500 \mathrm{~km}$. . . . . . . . . 150 


\section{List of Tables}

4.1 Parameters for the eddy-resolving and parameterized stress-driven channel experiments. . . . . . . . . . . . . . . . 75

4.2 Parameters for the eddy-resolving and parameterized spin-down experiments. 100

4.3 Parameters for the parameterized tropospheric eddy experiments. . . . . 111

5.1 Parameters for the eddy-resolving double-gyre experiment. . . . . . . 125 


\section{Chapter 1}

\section{Introduction}

This thesis addresses the challenge of adequately representing the transfer properties, and the forcing of the mean flow, by unresolved eddy processes in numerical ocean simulations.

Numerical models have become an integral part of trying to understand the physical state of the climate system and for predicting climatic change. The underlying reason for this dependence on model simulations is a lack of a general theory of climate. This thesis will not directly address climate. Rather it will focus on the parametric representation of one climatic process that impacts the mean state, and the temporal variability, of the atmosphere and ocean.

In the ocean, there are many scales of interest which contribute to its mean state. Due to our limited understanding of many of these processes and the limited speed of computers, it is not possible to include all of them in a single model. Physical processes are generally treated in one of three ways; they are either neglected, parameterized, or explicitly resolved. The questions being addressed in any given study should determine the physical processes which are necessary to include in the model, and therefore the necessary temporal and spatial resolutions in the model. However, in practice the available computer resources often dictate the resolutions used.

Variability in the current world ocean occurs over a wide band of frequencies and length scales, with the dominant energy containing scale being associated with quasi- 
geostrophic eddy variability (see section 1.1). These eddies are so-called because they are in approximate geostrophic balance. In addition, they manifest themselves at scales on the order of the local deformation radius. In the ocean, a typical Rossby radius of deformation is $50 \mathrm{~km}$, while the ocean basin domain size is typically $5000 \mathrm{~km}(\sim 100$ Rossby Radii). It is the representation of the transfer properties by the quasigeostrophic eddy field when it is not explicitly resolved, that is the focus of this thesis.

The representation of eddies in large-scale, state-of-the-art ocean models remains one of the outstanding computational challenge in ocean climate modeling. Climate studies demand integration of large-scale ocean dynamics globally and for time periods of thousands of years. Because of limited computational resources, the most highly resolved of such calculations are currently performed at $2^{\circ}$ resolution ( $\sim 4$ Rossby Radii) and so do not resolve the quasigeostrophic eddy field and its transport properties. To resolve the eddy field explicitly in models demands either that we embark on regional ocean circulation studies, or that we invest in global eddy-resolving numerical calculations that make rigorous demands on even the biggest and fastest computers available, see for example, Semtner and Chervin (1992). Therefore, for climate studies, the most appealing way forward is to parameterize through physical understanding, rather than resolve the transfers of heat, momentum, and vorticity on the eddy scale. Thus we see that there is currently a need for such parameterizations and they will continue to be exploited in the foreseeable future for long-term climate studies

Even though it is acknowledged that quasigeostrophic eddies in the ocean have an important effect on property transports, their unresolved representation in numerical ocean models has until recently remained very elementary indeed. Recent approaches to parameterization in coarse resolution ocean models have seemingly yielded much success. They have resulted in dramatic improvements to water mass distributions, a sharper thermocline, and a limiting of deep water formation to regions where it is known to occur. However, these studies concentrate only on the eddy transfer of tracer quantities and heat while maintaining crude representations for momentum and vorticity transport. 
They therefore fail to capture the full transfer by, and physics of, the quasigeostrophic eddies.

The approach of this thesis is to employ theoretical and numerical techniques to study the problem of more completely parameterizing the eddy fluxes of heat and momentum in terms of the large-scale flow. Ideas utilized by atmospheric dynamicists for midlatitude synoptic scale eddies are applied to their oceanic counterpart to render a more thorough understanding and representation of eddy transfer properties.

In order to motivate the problem in terms of its application to the real ocean, section 1.1 provides an overview of characteristics of eddy variability in the world ocean and the primary eddy production mechanism of baroclinic instability. In section 1.2, a review of previous approaches to the parameterization problem is presented with a consideration of their respective strengths and weaknesses. Finally, in section 1.3 the thesis research is introduced. Based on a solid dynamical framework, this study elucidates property transfer on the quasigeostrophic eddy scale and, therefore, provides a method of representing (through parametrization) the eddy transfer characteristics that is more complete, than has previously been offered for ocean eddies.

\subsection{Eddy variability in the ocean}

The early paradigm of a world ocean circulation consisting of a large-scale, laminar, and steady flow has been replaced by a turbulent picture of ocean variability on all space and time scales (see, for example, review articles by Wunsch (1981) and Schmitz and Luyten (1991)). It is now understood that ocean variability is broadband (occurring on all time and space scales) with a peak in the energy associated with mesoscale eddies, with timescales on the order of 100 days and length scales of order $100 \mathrm{~km}$ (Wyrtki et al. (1976); Dantzler (1977); Richman et al. (1977)). Wyrtki et al. (1976) show that for the North Atlantic, the ratio of eddy to mean energy is $\mathcal{O}(1)$ for the Gulf Stream region, while for the gyre interior this ratio is $\mathcal{O}(10)$ suggesting an intense, energetic 
eddy field with velocities of order ten times those of the mean flow. The importance of eddy transport of tracers and heat has been suggested by observations in the Antarctic Circumpolar Current (Bryden (1979)), the equatorial Pacific (Bryden and Brady (1989)), and the North Atlantic (McWilliams et al. (1983)). Momentum transport by the eddies has also been documented to be important, particularly in the intense current regions. Webster (1965) showed that Gulf Stream eddies act as an energy source for the mean current, transferring momentum from the flanks of the Gulf Stream to the center of the jet. Hogg (1993) analyzed moored current arrays and determined that eddy vorticity fluxes were capable of driving two counter-rotating recirculation gyres either side of the Gulf Stream.

Evidently, these mesoscale eddy motions are an important physical process in the ocean, and there is an obvious need for parameterization if they are not adequately resolved. A logical way forward would be to base a parameterization on physical understanding of eddy dynamics. Unfortunately, because of sparse data coverage in the ocean, the dynamics of eddies and their associated transport properties have not been well understood. However, recent studies using altimetry data (Stammer and Wunsch (1994), Wunsch and Stammer (1995), Stammer (1997)) have provided much insight into global characteristics of ocean variability. These studies suggest that the variability is due to an instability process of the large-scale mean flow.

Gill et al. (1974) present simple energetic arguments in a two-layer model which demonstrates that there is sufficient potential energy stored in the mean density field to account for the observed eddy kinetic energies. The method of potential energy release that the authors advocate is in situ baroclinic instability of the mid-ocean. This mechanism was also proffered for mid-ocean energy release by Robinson and McWilliams (1974). Gill et al. (1974) argue that the ratio of available potential energy to mean kinetic energy in the mid-ocean gyres is given by $\left(L / L_{D}\right)^{2}$, where $L$ is the lengthscale of the gyre, and $L_{D}$ is the Rossby radius of deformation. Hence the available potential energy of the wind-driven circulation exceeds the mean kinetic energy by a factor of $\mathcal{O}(1000)$. 
Whether the available potential energy is released through in situ baroclinic instability is still unclear and has been the subject of much debate. Pedlosky (1975), using theoretical arguments, points out that an upper bound for the eddy velocity produced by baroclinic instability is the velocity of the large-scale mean flow. He suggests (Pedlosky (1977)) a plausible, alternative mechanism of eddy energy penetration into the gyre interior; eddy generation occurring in the intense current regions and then radiation of this energy into the far-field of the gyre interior.

Other mechanisms have been put forward to explain the observed ocean variability. Firstly, direct wind generation of ocean eddies is possible in regions of high atmospheric forcing. Second, Philander (1978) shows that a wind-induced barotropic variability should be enhanced at high latitudes because of the weaker stratification and thus deeper penetration scale. Thirdly an alternative mechanism is mean flow interaction with topography (e.g. Bretherton and Karweit (1975)). Lastly, barotropic instability generated through horizontal shears in the boundary current regions is another possibility. However, it seems that baroclinic instability is the primary mechanism for the global patterns of variability and using ideas from baroclinic instability theory allows us to gain an understanding of the length and time scales of the oceanic eddy motions.

\section{Eddy length, and time scales}

The process of baroclinic instability has been the subject of a great many years of research. For a comprehensive review see Pierrehumbert and Swanson (1995). The pioneering work in the subject was done by Charney (1947) and Eady (1949). They used normal mode analysis to demonstrate that the structure of vertical modes could explain the existence of midlatitude cyclones in the atmosphere in terms of the instability of a baroclinic zonal current to infinitesimal wave disturbances.

One description of the baroclinic instability mechanism is that of a 'wedge of instability', see, for example, Pedlosky (1987). Differential heating in the atmosphere establishes a meridional temperature gradient between the equator and the pole. As the earth is 


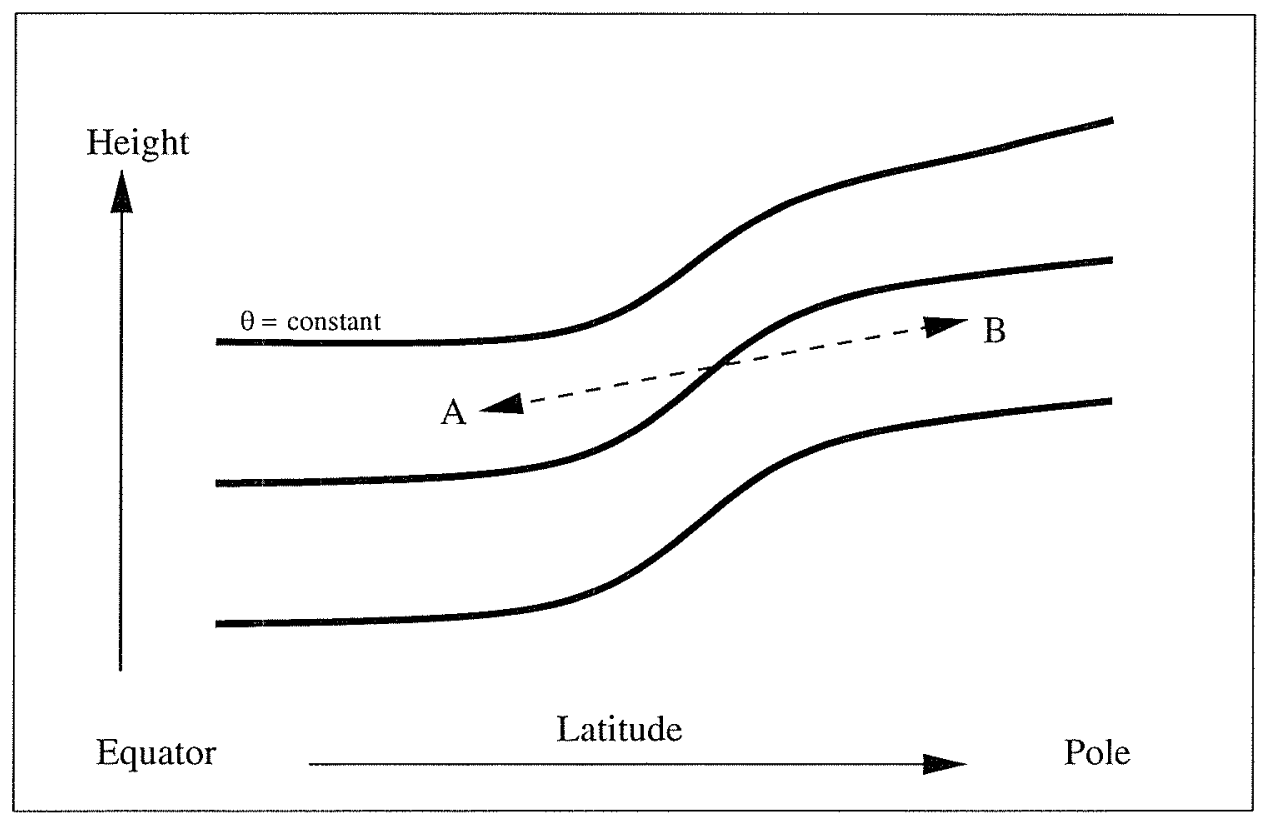

Figure 1.1: A schematic picture of the 'wedge of instability' description of baroclinic instability for the troposphere. Potential energy of the mean flow is released for the trajectory $\mathrm{AB}$ relative to the isopycnal slopes.

rotating, geostrophic balance occurs where the resultant pressure gradient is balanced by the Coriolis force on westerly jets at midlatitudes in each hemisphere. The jets are said to be in thermal wind balance with the vertical shear of the flow being proportional to the meridional temperature gradient. As Figure 1.1 shows, the sloping temperature surfaces suggest a reservoir of potential energy. If a fluid parcel moves along the trajectory $A B$, it can release energy from this reservoir by transporting heat polewards and upwards. Simple analysis of the buoyancy forces shows that the parcel on such a trajectory will. be further accelerated from its original position. This is the basic mechanism of baroclinic instability. However, this description can be slightly misleading as constraints on the fluid motion can prevent the balanced motion from following trajectories that further accelerate the initial displacement. Consequently the flow must satisfy certain criteria before instability can occur. The Charney-Stern theorem states that for flow (bounded 
in the vertical) to be unstable the meridional potential vorticity gradient must change sign in the flow domain. For flow not bounded in the vertical the necessary criterion for instability is that the meridional gradient of potential vorticity in the flow is opposite to the meridional gradient of the potential temperature at the lower boundary. ${ }^{1}$

The waves generated through baroclinic instability are required to have horizontal extent greater than the local deformation radius $L_{D}$ in order to release potential energy from the mean flow. However length scales much larger than $L_{D}$ result in an inefficient energy release (Pedlosky (1987)). Therefore horizontal scales on the order of the local deformation radius $L_{D}$ are preferred for the eddy motions generated by baroclinic instability.

The $e$-folding growth rate $\sigma$ of the baroclinic waves in the Eady study scales as

$$
\sigma \sim \frac{f}{\sqrt{R i}},
$$

where $f$ is the Coriolis parameter and $R i$ is the Richardson number of the large-scale flow:

$$
R i=\frac{N^{2}}{\left(\left(u_{z}\right)^{2}+\left(v_{z}\right)^{2}\right)},
$$

where $N^{2}$ is the square of the buoyancy frequency and $(u, v)$ are the horizontal components of the geostrophic mean flow. The Richardson number is measure of the relative importance of the buoyancy to inertial terms in the flow and is essentially a measure of the ratio between the potential and kinetic energies.

In figure 1.2 we plot a vertical average, over the upper $2000 \mathrm{~m}$ of the ocean, of the Eady growth rate, $\sigma$, for the upper $2000 \mathrm{~m}$ of the ocean computed by

$$
\sigma=\frac{f}{H} \int_{-H}^{0} \frac{1}{\sqrt{R i}} d z
$$

\footnotetext{
${ }^{1}$ In quasigeostrophic theory, the lower boundary potential temperature gradient can be shown to be equivalent to a delta-function sheet of potential vorticity just interior to the boundary. The necessary condition for instability can therefore be couched in terms of potential vorticity alone. Use will be made of the equivalence of boundary temperature perturbations and interior potential vorticity distributions throughout the thesis.
} 


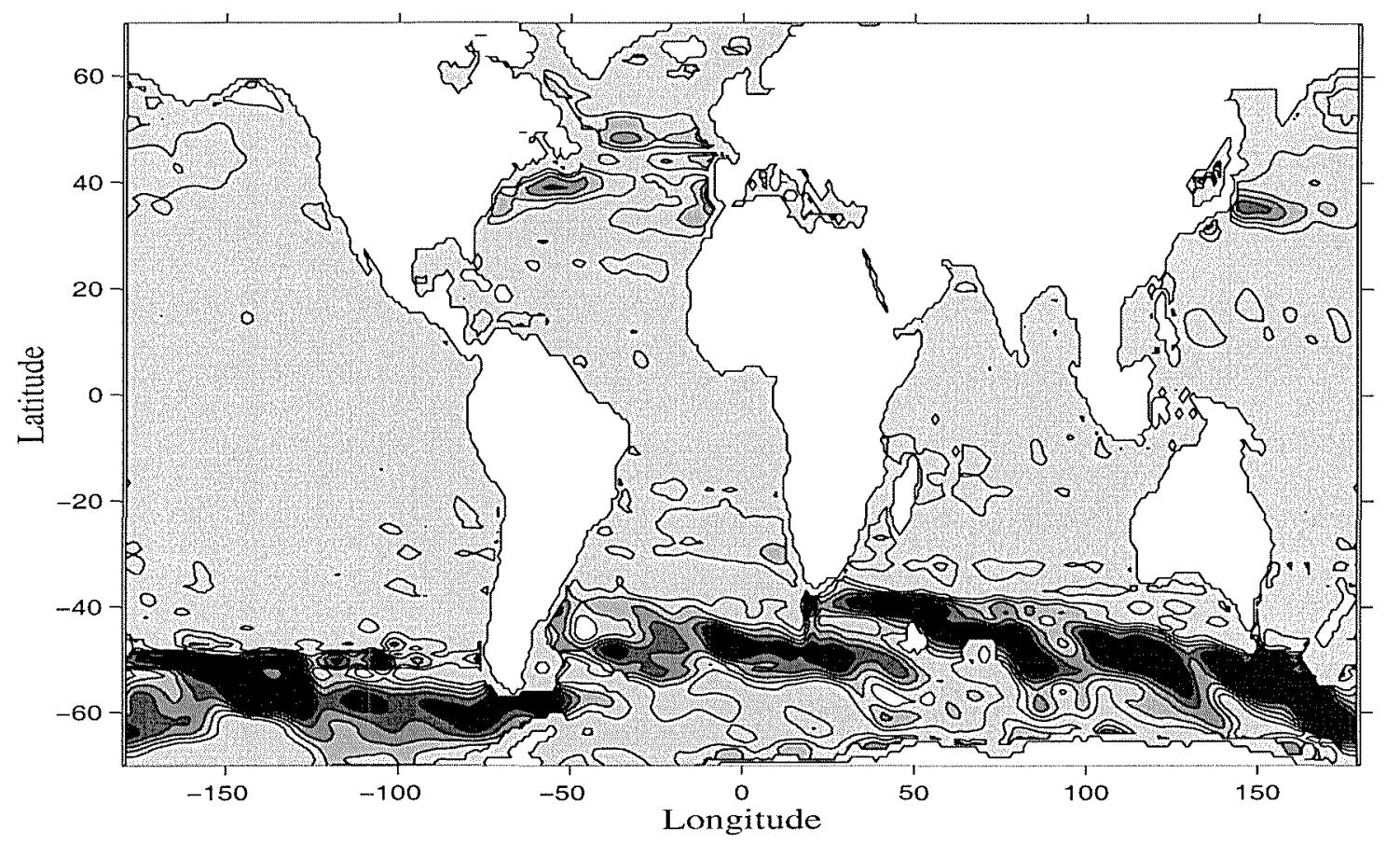

Figure 1.2: The Eady growth rate, $\sigma$ calculated using (1.3) from Levitus (1994) annual climatology. The integral is computed from 2000 to $100 \mathrm{~m}$. The contour range is $1 \times 10^{-6}$ $\mathrm{s}^{-1}$ to $5 \times 10^{-6} \mathrm{~s}^{-1}$ in intervals of $1 \times 10^{-6} \mathrm{~s}^{-1}$. The fastest growth rates are present in the western boundary currents of the North Pacific and Atlantic basins and in the Southern Ocean.

from the Levitus (1994) annual climatology. The $\sigma$ distribution is spatially inhomogeneous with regions of intense growth of baroclinic waves. The buoyancy frequency can be considered to be constant to first order. Thus spatial variations in $\sigma$ are due to both the change in the Coriolis parameter with latitude and due to horizontal gradients in the vertical shear and therefore horizontal density gradients in frontal regions. From figure 1.2 we see that the fastest oceanic growth rates are present in the western boundary currents of the North Pacific and Atlantic basins and in the Antarctic Circumpolar current.

Figure 1.3 plots the rms sea-surface height (ssh) variability averaged over 4 years obtained from the TOPEX/POSEIDON satellite altimeter. Intense variability can be seen in regions of strong boundary currents. Comparison with Figure 1.2 highlights a 


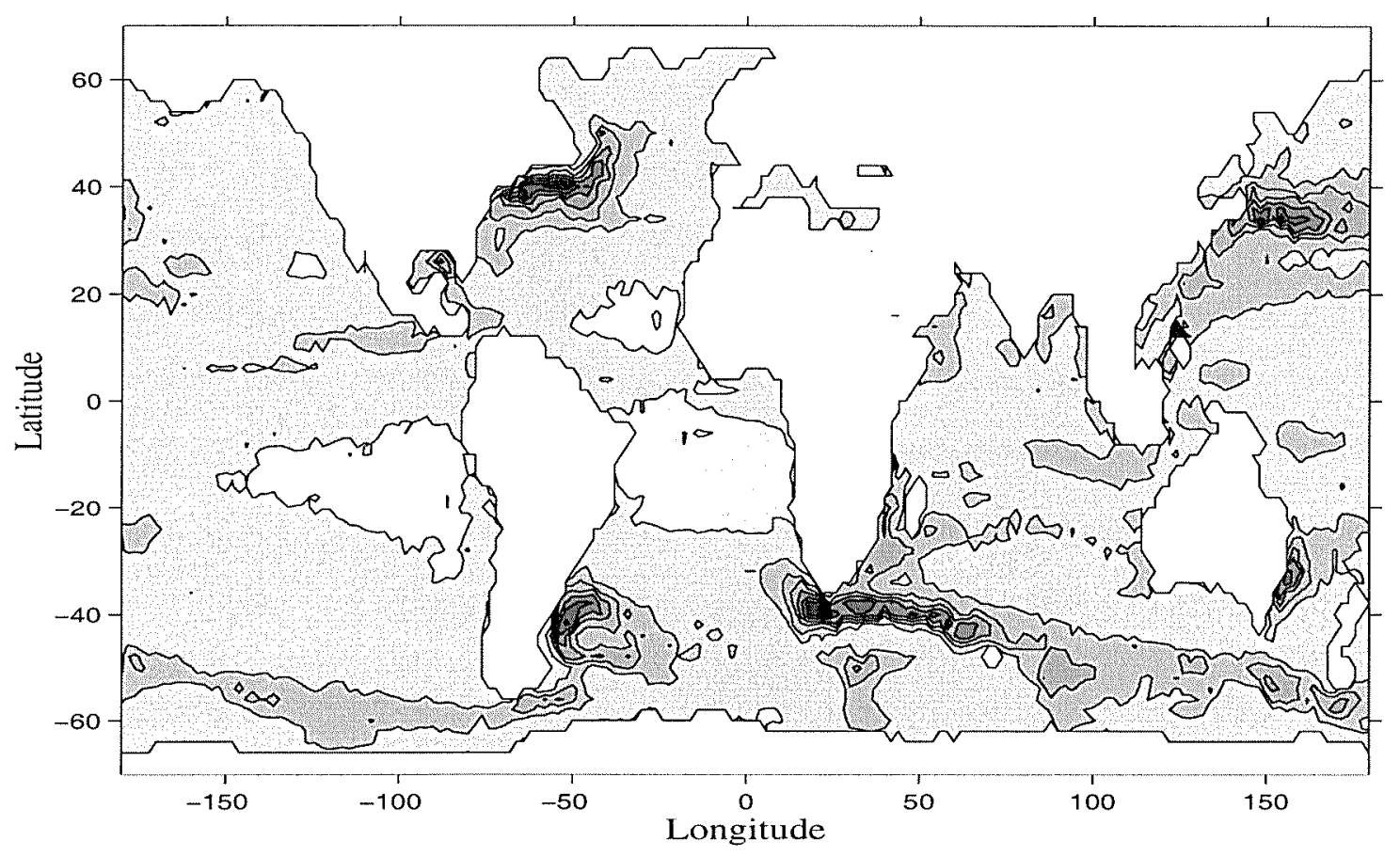

Figure 1.3: TOPEX/POSEIDON rms sea-surface height (ssh) variability averaged over 4 years (courtesy C. King, MIT). The range contoured is 0 to $30 \mathrm{~cm}$ in intervals of $5 \mathrm{~cm}$. There is marked variability in the western boundary currents of the Pacific and Atlantic basins and in the Southern Ocean.

broad agreement between the locations of observed ocean variability and of computed eddy growth rate. This agreement suggests that mesoscale variability is closely related to the baroclinicity of the large-scale flow.

Figures 1.2 and 1.3 demonstrate that eddies generated through baroclinic instability are ubiquitous in the global ocean and have characteristic time-scales of weeks to months, and space-scales of tens of kilometers. Although referred to as mesoscale eddies in the oceanographic literature, these turbulent eddies in near geostrophic balance are analogous to synoptic eddies in the atmosphere, whose time scales are hours to days and spatial scales are of thousands of kilometers. This analogy will prove to be advantageous as the thesis will show.

It is clear that if one wishes to use a numerical model to calculate the dynamics 
and water mass distribution of the large-scale ocean circulation, the eddy transporting properties of tracers and momentum must be incorporated into the calculation.

\subsection{Prior representation of eddy transfer}

A tremendous body of work has been dedicated to understanding and parameterizing transfer properties and mean flow forcing by eddy processes in both the atmosphere and ocean. I choose to give a brief overview here.

\subsubsection{Atmospheric eddies}

In the atmosphere, eddies counteract the latitudinal imbalance of radiative heating by transporting heat polewards. Jeffreys (1926) showed that large-scale eddies are responsible for midlatitude surface wind maintenance by considering the momentum budget of latitude bands. Special attention was attached to understanding the surface winds of the earth, since they are related through frictional divergence to locations of high and low precipitation. Moreover, the vertical structure of the divergence of the eddy momentum flux along with surface friction is responsible for the maintenance of the Hadley, Ferrel, and Polar cells observed in the Eulerian mean. For these reasons atmospheric dynamicists, in stark contrast to their counterparts in oceanography, have concentrated on the eddy transport of momentum in particular.

Atmospheric transport of momentum, heat and chemical constituents has been frequently represented as a downgradient diffusion process, with positive diffusion coefficients. 'Mixing length' ideas were obtained by likening the geophysical eddy transfer process to eddy transfer in the kinetic theory of gases. The mixing length hypothesis assumes that a displaced fluid parcel will carry its properties for a characteristic length scale and then mix with the surrounding fluid. This is analogous to a gas molecule traveling a mean free path distance before exchanging momentum with another molecule upon 
collision. Using mixing length theory, the eddy flux terms are represented as

$$
\overline{v^{\prime} \tau^{\prime}}=-\kappa \nabla \bar{\tau}
$$

for a conserved quantity $\tau$. This is Ficks first law for diffusion, and leads to the terminology of Fickian diffusion for any process represented in this manner. In general the transfer coefficient, $\kappa$, has differing values between the horizontal and vertical. Eventually, mixing length theory of momentum transfer had to be forsaken for large-scale atmospheric flows because the implied eddy transfer coefficient was negative (Starr (1968)).

The mixing length approach was applied to potential vorticity by Green (1970) who argued that for the conserved quantity - potential vorticity - the eddy field acts to transfer potential vorticity down the large scale gradient. He further noted that mixing length arguments had been incorrectly applied to momentum. A parcel of fluid in the atmosphere does not conserve its momentum, due to the presence of pressure gradient forces. Thus, it is not appropriate to represent momentum transfer by baroclinic eddies as a diffusion process, since the parcel velocity has undergone changes during the displacement.

Green (1970) represented the physics of the transfer process by prescribing the shape and magnitude of the transfer coefficients. The eddy transfer characteristics of momentum and heat are dependent upon the anisotropic nature of the eddies. The sense of the Reynolds stress terms depends upon the variation of the trough and ridge lines of the amplifying waves. A tilt of the trough lines with height gives a horizontal heat transport, and a bending of the trough lines in the horizontal produces momentum transport. The spatial anisotropy of the eddies was revealed by linear baroclinic instability theory, and this helped Green (1970) specify the spatial form of the eddy transfer coefficients. He then prescribed their magnitude from energetic arguments. In a similar manner to Green (1970), Stone (1972) drew on insights from linear baroclinic instability to parameterize the eddy heat fluxes in a radiative-dynamical model of atmospheric stratification. Studies of parameterization in the atmosphere really end here. The eddy length scale in the atmosphere is on the order of $1000 \mathrm{~km}$, about $1 / 10$ the domain size. Thus it is relatively 
easy, compared to the ocean case, to resolve the geostrophic eddy field in models of the atmosphere. Thus eddy parameterization on the synoptic scale in the atmosphere is necessary only for coupled ocean-atmosphere climate integrations of thousands of years.

However, the understanding of atmospheric eddy motions and their impact on zonal mean flows has taken an enormous step forward in the last two decades. We have been presented with practical, powerful theorems which furnish us with diagnostic methods to deal with eddy forcing and propagation in zonal flows [Eliassen and Palm (1961), Andrews and McIntyre (1976,1978a)]. Further, generalized Lagrangian mean (GLM) theory [Andrews and McIntyre (1978a,b), McIntyre (1980)] describes the interaction of the eddies with the zonal mean flow and provides a clearer description of the eddy forcing. This approach shows that the eddy flux term can act in an advective manner similar to a Stokes drift velocity which is due to the anisotropy of the amplifying wave field. In their paper, Eliassen and Palm (1961) laid down the foundations for this approach. They introduced a quantity that involves northward fluxes of heat and zonal momentum in the meridional plane, showing that the eddy forcing of the zonal mean circulation is given by the divergence of what was later named the Eliassen-Palm flux. These studies have led to useful theoretical tools which have been applied diagnostically to atmospheric data [see, for example, Edmon et al. (1980), Palmer (1981)] to provide clear insight into eddy transfer characteristics and their feedback on the atmospheric zonal mean flow. Moreover, in the transformed Eulerian mean formulation, which is based upon GLM theory, the nonacceleration theorem of Charney and Drazin (1961) is transparent, while such a result is not at all obvious in the conventional untransformed Eulerian mean approach.

These useful ideas for zonal mean flows have been extended to time-mean flows [Hoskins (1983); Hoskins et al. (1983); Trenberth (1986); Plumb (1986); Andrews (1990)] to give insight into the eddy propagation statistics and forcing of non-zonal flows. Plumb (1990) further clarified the picture of forcing of the mean flow by the eddies by deriving a nonacceleration theorem for quasigeostrophic eddies on a time mean flow. 
From these studies it is clear that the atmospheric dynamicists have a deeper understanding of eddy transfer, propagation, and forcing of the atmospheric zonal and time mean flows, which incorporates both the heat and momentum eddy transport.

\subsubsection{Ocean eddies}

There have been many attempts and methods used to incorporate the transfer of properties by eddies in non-eddy resolving ocean models. Here, they will be grouped into two categories; the approaches of Fickian diffusion and bolus velocity transport.

\section{Fickian diffusion}

The first approach is the common practice of incorporating the effect of eddies as Fickian diffusion, based on mixing length arguments, for both momentum and tracers. This is physically inadequate on two counts. First, mixing length arguments are unjustified in the case of momentum [Webster (1965), Starr (1968), Green (1970)], and second, eddy tracer transport can act as an advective process (Plumb and Mahlman (1987)). Prior to 1990 this was the method of incorporating the eddy effects into coarse resolution ocean models. Even the most sophisticated schemes concentrated on tracer diffusion. Cox (1987) mixed the tracer along isopycnal surfaces by rotating a diffusion tensor such that the large diffusive flux is along isopycnal surfaces (Redi (1982)). However, Cox (1987) was still obliged to run his model with a small background Fickian diffusivity in order to prevent numerical instability. This has profound implications for climate simulations, because they are integrated for thousands of years, a time scale over which any small amount of diffusivity will become important. Any diffusive parameterization will contribute to the overly diffusive nature of ocean models, leading to a problem of preserving tracer quantities and distributions in such simulations.

A further important drawback to the diffusive representation is that we may be studying a different dynamical system in the models compared to that which exists in nature. The real climate system can undergo transitions, either abrupt or over long time scales, 
to different states. Lorenz (1968) argued that so called "almost intransitive" non-linear models could, in like manner, exhibit natural oscillations and reach equilibria that are to some extent determined by the initial conditions. However, highly diffusive models tend to be "transitive", meaning that unique equilibrated states are reached that are independent of the initial conditions. Hence excessively diffusive models are transitive and are arguably not the appropriate tool for studying the inherently variable ocean climate system.

Exceptions to the rule of diffusive representation were the potential vorticity mixing theories of Welander (1973) and Marshall (1981), and the homogeneous turbulence study of Haidvogel and Held (1980).

Marshall (1981) applied Green's (1970) ideas to a zonal mean ocean, such as in the Antarctic Circumpolar Current (ACC). They were further applied to a three-dimensional barotropic gyre flow by Marshall (1984). In these studies, potential vorticity was transferred down its mean gradient; upgradient momentum transfer resulted in some regions, sharpening the eastward jet of the ACC for example. Although successful, Marshall's (1981) approach is not of immediate use in climate modeling. The study was undertaken in a two layer fluid governed by the quasigeostrophic potential vorticity equation. However, most numerical models do not have potential vorticity as a prognostic variable, so unless an additional inversion were undertaken - creating additional computational expense - a potential vorticity transfer approach in the conventional Eulerian framework is not possible.

\section{Bolus velocity transport}

In the second type of approach, Gent and McWilliams (1990) addressed the diffusive nature of ocean models by likening the effect of the eddies on the transport of tracers to that of a Stokes advection. This work was presented without reference to, or knowledge of, the tropospheric studies of Andrews and McIntyre (1976,1978), and Plumb and Mahlman (1987), among many others. Only recently have the authors reconciled their approach 
with the atmospheric literature [see Gent and McWilliams (1996)].

For coarse-resolution ocean model flows, Gent and McWilliams introduce an "effective advection velocity", consisting of the large scale velocity and an "eddy-induced" or "bolus" velocity that depends on the density flux of the eddies, which replaces the large scale velocity in the tracer equation.

Note: throughout the thesis the nomenclature of "eddy-induced velocity" is avoided to prevent confusion over the role of the eddies in forcing the mean flow. It incorrectly implies that the meridional residual circulation can be separated into two terms; one of which is the Eulerian mean circulation and is independent of the eddy disturbances and one that is the sole result of them. However, the eddies can and do modify the mean circulation through their momentum and heat transport. Hence, there is actually an "eddy-induced" component in the Eulerian mean circulation. It is therefore wise to avoid this artificial and inaccurate separation. As a result, we prefer to think in terms of the "residual mean velocities" which are the sum of the Eulerian mean velocities and terms which depends on the divergence of the horizontal heat and momentum fluxes.

The Gent and McWilliams (1990) (GM) technique consists of mixing along isopycnal surfaces of the thickness between adjacent isopycnals, while conserving the volume of fluid between such surfaces. This mixing of isopycnal thickness implies the dynamical effect of the depletion of available potential energy (and the vertical transfer of momentum), mimicking baroclinic instability.

The scheme was first implemented in McWilliams and Gent (1994) in a balanced equations model. Gent et al. (1995) presented the scheme in such a manner that it could be implemented in a $z$-coordinate numerical model. Danabasoglu et al. (1994) and Danabasoglu and McWilliams (1995) introduced the GM scheme into the GFDL MOM model and found that it allowed for a drastically reduced value of lateral diffusivity, alleviating to a certain extent the highly diffusive nature of numerical ocean models.

Subsequent work on ocean eddy parameterization since GM has concentrated on prescriptions for the "bolus" velocity. These studies assume a release of mean potential 
energy through baroclinic instability. Most techniques are guided by the concept of the depletion of available potential energy and eddy fluxes that are preferentially directed along isopycnal surfaces. Some authors introduce additional complications relating to neutral surfaces [Hirst and McDougall (1996), McIntosh and McDougall (1996), McDougall and McIntosh (1996a,b)] or stochastic theory [Dukowicz and Greatbach (1997)]. Tandon and Garrett (1996) focus on the "bolus" velocity by discussing the impact on it when eddy energy dissipation is local in nature due, for example, to a process such as the breaking of internal waves. Treguier et al (1997) considered how the constraints on the "bolus" velocity are impacted by the presence of horizontal boundaries. They also noted that potential vorticity and not isopycnal thickness was a more appropriately conserved quantity, a point also noted by Marshall et al. (1998) and Lee et al. (1997). Visbeck et al. (1997) examine the Gent and McWilliams scheme in the light of four eddy resolving calculations and find that the most appealing results are obtained when Green's (1970) transfer theory is used to specify the transfer coefficient for the isopycnal heat flux which in turn specifies the "bolus" velocity.

Recently Killworth $(1997,1998)$ has offered an alternative parameterization scheme to GM, but like GM, his focus is on eddy tracer transport. His scheme is based on potential vorticity and isopycnal thickness fluxes in linear baroclinic instability. Killworth (1998b) tests the scheme in an eddy-resolving channel and not surprisingly finds his parameterized results are identical to results obtained by employing the GM scheme. Greatbach (1998) recently offered an interesting paper which, like this thesis, advocates a potential vorticity flux term in the momentum equation. However, his study differs from my thesis in three respects. Firstly, in the governing equations of Greatbach (1998), the velocities that appear are a mixture of those of the Eulerian mean and a "tracer transport velocity". This leads to difficulties for prognostically calculating the flow evolution. Second, he neglects the flux of relative vorticity (lateral momentum transfer) by the eddies and in so doing overlooks an important component of the physics of the eddy process. Thirdly, the scheme is not implemented in a numerical model. 


\section{Notable shortcomings of the previous oceanographic studies}

Based on the review presented above, I take the following to be true for the remainder of the thesis:

1. The Fickian diffusion representation of eddy tracer has led to non-eddy-resolving numerical ocean models being overly diffusive. Further, a diffusive picture of heat and tracer transfer may be inappropriate because eddy transport may be advective in nature.

2. The Fickian diffusion representation of eddy momentum transfer is clearly in error.

3. Recent studies, although producing success in mimicking the main features of water mass distributions, have neglected the physics of the momentum transfer entirely. They have chosen to concentrate on the eddy transfer of tracers. These studies therefore lack the ability to incorporate the total eddy effects on water mass distributions and the ocean mean flow.

There are two logical conclusions one can reach upon reading the above points. First, improvements to eddy representation in non-eddy resolving ocean models can readily be made. Alternatively, one could argue that to attempt to try would be a hopeless endeavor. I side with the first inference, and the resultant work follows.

\subsection{Focus and overview of the thesis}

The focus of this thesis is to tackle the difficult problem of the representation of quasigeostrophic eddies in non-eddy resolving, hydrostatic, primitive equation (HPE) models. The goal is straightforward; to obtain a more complete framework to incorporate the eddy tracer and momentum transfer. There are those who may argue that even though the intense variability shown in figure 1.3 is present, it may have little or no dynamical consequence in the ocean. Although this may be the case, I feel that there is a certain virtue in deriving a parameterization approach that is more physically based than those 
in current use, and then exploring the scheme in light of the eddy physics. The method presented has at its core the physics of the transfer of potential vorticity.

In quasigeostrophic models a framework is strongly suggested by the potential vorticity theorem. The heat and momentum aspects of the eddy transfer process can then be naturally combined by phrasing them in terms of potential vorticity transport - see, for example, Marshall (1981). Eddy closure, although still thwart with difficulties, is at its most transparent. However the ocean is not quasigeostrophic; it is inappropriate, for example, to linearize the thermodynamic equation about a constant reference stability profile which must be held constant in the horizontal. How, then, can progress be made in more complete models?

A potential vorticity theorem exists for the HPE equations, the starting point of most ocean climate models. However, to invert for the flow field requires specification. of a balance condition (i.e. geostrophic balance) and a reference state. This would lead to major complications in the treatment of the lateral boundaries, the loss of gravity-wave dynamics, and increased computational expense! Further, unlike quasigeostrophic models, ocean climate models do not have potential vorticity as the the prognostic variable. In the HPE models, momentum and temperature are stepped forward separately and the effect of the eddies (eddy momentum and heat flux divergences) appear as forcing terms on the right-hand-side of the equations and are subsequently parameterized separately. It is argued here that this separation of the heat and vorticity transporting properties of eddies - a separation that is dictated largely by algorithmic, rather than physical considerations - significantly complicates the parameterization problem and, if possible, should be avoided.

The thesis presents an alternative way forward in which the full HPE's are transformed, guided by the formalism of the "transformed Eulerian mean" of Andrews and McIntyre (1976). In the mean equations, and only if the eddies are assumed to be quasigeostrophic, their effect appears as a body force term - an eddy potential vorticity flux driving the momentum equations. Parameterization can then focus on the closure of this 
flux. This task is easier, due to the quasi-conserved nature of potential vorticity, than parameterizing the transfer of heat and momentum separately.

Chapter 2 presents the dynamical framework of the approach. It details how eddy effects can be written symbolically by a flux of potential vorticity. Chapter 3 presents the closure assumption to be used for this eddy term. In chapter 4 the approach is implemented in a numerical model. Mean fields and transfer characteristics from a parameterized model are compared to those from an eddy resolving model for zonal mean flows. The advantage of this approach is further illustrated with application of the parameterized model to an atmospheric problem. In chapter 5, we extend our scope to consider flows where a spatial mean may not be appropriate. The time mean theory presented in chapter 2 is investigated in the light of an eddy resolving ocean calculation in a geometry where there is no obvious spatial symmetry. In chapter 6 , we review the major results of the thesis and discuss possible future directions of the work. Appendix A presents the details of the numerical model used. 


\section{Chapter 2}

\section{The Dynamical Framework}

\section{$2.1 \quad$ Introduction}

This chapter presents and details the theoretical framework used throughout the thesis. This framework differs from that which is ingrained in the dynamical oceanography community, which stresses the conventional Eulerian mean. I will make use of an alternative, and conceptually more appealing, coordinate system, that is, unfortunately, not familiar to most oceanographers.

Section 2.2.1 briefly reviews the differing formulations employed in dynamical studies of atmospheric zonal flows. These theories have been extended to cover time mean flows, but to avoid confusion for someone not reasonably familiar with the subject, the review covers only zonal mean theories. This will not lead to any loss of understanding. Section 2.2.2 provides a necessary revision of quasigeostrophic potential vorticity and eddy fluxes of potential vorticity in order to effectively present material later in the chapter. Section 2.3 presents the well known Eulerian mean formalism and illustrates clearly why this is not the most effective way in which to look at eddy transfer. In sections 2.4 and 2.5 , I introduce the formalism that will be used throughout the thesis. It will be used both diagnostically and prognostically to determine eddy propagation, transfer, and forcing of the mean flow in the later chapters. Section 2.6 discusses the potential vorticity flux 
representation, commenting on the form it takes and on issues involving momentum constraints. Section 2.7 states the governing equations for time mean flows. Finally, in section 2.8 the governing equations for zonal mean flows are presented.

\subsection{Background}

\subsubsection{Zonal mean coordinate systems}

For the benefit of any readers who have not encountered the atmospheric eddy studies outlined in the preceding chapter, I choose to briefly review different mean formalisms commonly used in the atmospheric literature for studying zonally symmetric flows.

\section{Eulerian zonal mean}

The most conventional and straightforward approach is to simply zonally average the governing equations of motion. Eddies are defined as the deviation of a quantity from its zonal average. Reynolds averaging leads to the eddies appearing explicitly as eddy flux (correlation) terms in the equations for the mean quantities. The eddy momentum and tracer terms appear in separate equations.

\section{Transformed Eulerian mean}

To investigate eddy forcing of the mean flow, it has proved advantageous to transform the equations of motions to a more convenient form. This was originally done by Andrews and McIntyre (1976) to yield altered equations known as the "transformed Eulerian mean" (TEM). In the TEM theory, the mean velocities in the meridional plane are redefined using the eddy temperature flux term to give what is known as the "residual mean circulation".

The advantages of the TEM formalism are twofold. First, the eddy correlations appear only as one term - the divergence of the "Eliassen-Palm flux" in the zonal momentum 
equation. This is much clearer than the Eulerian mean approach, in which multiple correlation terms appear. The occurrence of only one eddy term emphasizes the fact that eddy fluxes of heat and momentum act in combination and not separately. Second, the tracer advection is by the "residual mean circulation", which under certain assumptions is equal to the "effective transport velocity" defined by Plumb and Mahlman (1987). This is the relevant velocity for understanding atmospheric tracer transport in the meridional plane.

\section{Generalized Lagrangian mean}

The TEM formulation is derived assuming that the eddies are of small amplitude, a restriction often broken when they grow to finite amplitude. To avoid these difficulties, Andrews and McIntyre (1978a,b) developed a mean coordinate system concerned with the interaction of the eddy disturbances and the mean flow which is exact. This revealing conceptual definition of the mean is known as the "generalized Lagrangian mean" (GLM). The procedure is to average following fluid parcels, rather than over a fixed spatial region. Although the theory is appealing from a conceptual point of view, serious practical difficulties are encountered when attempting direct application to atmospheric flows. As a result the theory has seen little practical use since its development.

\subsubsection{Quasigeostrophic PV, PV sheets, and eddy PV fluxes}

\section{Quasigeostrophic PV}

Potential vorticity (PV) is an indispensable tool for understanding most aspects of largescale oceanographic and atmospheric flows [this is stressed in textbooks e.g., Pedlosky (1987), and review articles e.g., Hoskins et al. (1985), and Rhines (1986)]. Quasigeostrophic motions are geostrophically balanced flows on a beta plane in a stratified fluid. The quasigeostrophic PV equation

$$
\frac{D q}{D t}=0
$$


governs the evolution of the quasigeostrophic PV, $q$

$$
q=f_{0}+\beta y+\frac{\partial v}{\partial x}-\frac{\partial u}{\partial y}+f_{0} \frac{\partial}{\partial z}\left(\frac{T}{\partial \bar{T} / \partial z}\right)
$$

where $u$ and $v$ are the geostrophic velocities, $T$ is the temperature, and $D / D t=\partial / \partial t+$ $u \partial / \partial x+v \partial / \partial y$ is the substantial derivative following the geostrophic flow. Using the geostrophic and hydrostatic balance relations we can write $u, v$, and $T$ in terms of the geostrophic streamfunction, defined by

$$
\psi=\frac{1}{\rho_{0} f_{0}}\left(p-p_{0}\right)
$$

where $p_{0}(z)$ is a suitably defined horizontal mean reference pressure profile, $p_{0}(z)=\bar{p}^{x y}$, and $\overline{(}^{x y}$ denotes an average over $\mathrm{x}$ and $\mathrm{y}$. This allows us to write the geostrophic velocities as

$$
u=-\frac{\partial \psi}{\partial y} \quad ; \quad v=\frac{\partial \psi}{\partial x} \quad ; \quad w=0
$$

and hydrostatic balance thus:

$$
T=\frac{f_{0}}{g \alpha} \frac{\partial \psi}{\partial z}
$$

The quasigeostrophic PV can be written in terms of the geostrophic streamfunction, viz:

$$
q=f_{0}+\beta y+\frac{\partial^{2} \psi}{\partial x^{2}}+\frac{\partial^{2} \psi}{\partial y^{2}}+f_{0}^{2} \frac{\partial}{\partial z}\left(\frac{1}{N^{2}} \frac{\partial \psi}{\partial z}\right)
$$

with $N^{2} \equiv N^{2}(z)=g \alpha{\overline{T_{z}}}^{x y}$ being the square of the horizontal mean buoyancy frequency. Equation 2.1 is termed the quasigeostrophic PV equation because the evolution of $q$ is determined by the ageostrophic effects of time dependence, advection, changes in planetary vorticity, and vortex stretching.

Fluid parcels conserving their PV (equation 2.1) in the presence of an ambient planetary vorticity gradient $(\beta)$, experience a restoring force which leads to Rossby wave propagation. If one of these waves propagates in close proximity to another, they can mutually interact, draw energy from the mean flow, and make possible the linear barotropic and/or baroclinic shear instabilities responsible, as discussed in chapter 1, for much of the eddy motions in the atmosphere and ocean. 
For atmospheric zonal flows, Charney and Stern (1962) showed that that for vertically bounded flow to be unstable the meridional potential vorticity gradient must change sign somewhere in the flow. For flow without an upper boundary the necessary criterion for instability is that the meridional gradient of potential vorticity in the flow is somewhere opposite to the meridional gradient of the potential temperature at the lower boundary. In the earth's atmosphere the PV gradient is always positive in the troposphere, while the poleward temperature gradient is always negative. Thus, the Charney and Stern (1962) necessary condition for baroclinic instability is always met for large-scale atmospheric flows. However, the eddies do not grow unrestrained, via continuous extraction of energy from the mean flow. The eddy growth is checked by eddy dissipation. Either nonlinear processes dissipate eddy energy at the same rate that energy is extracted from the mean flow, or the eddies alter the mean flow to modify their growth rates until dissipation is matched.

\section{PV Sheets}

With knowledge of the PV, the streamfunction $\psi$ can be determined by inverting the elliptic problem given by equation 2.6. The boundary conditions can be inferred from kinematic conditions at the boundaries. At the upper and lower boundaries there exists an established temperature distribution. Equation 2.5 provides inhomogeneous Neumann boundary conditions on $\psi$ at the horizontal boundaries since $\partial \psi / \partial z$ is specified there. A computational and conceptual simplification can be made if we replace the inhomogeneous Neumann boundary conditions by homogeneous ones. Drawing on insights from potential theory, Bretherton (1966), working within the confines of quasigeostrophic theory, recognized that boundary temperature distributions are mathematically equivalent to concentrated sheets of quasigeostrophic PV just interior to the boundaries, if those boundaries are then assumed to be isothermal. This enables us to incorporate the boundary temperature distributions as parts of the interior PV distribution.

We define the quasigeostrophic PV $\tilde{q}$, which is equal to $q$ in the fluid interior, except 

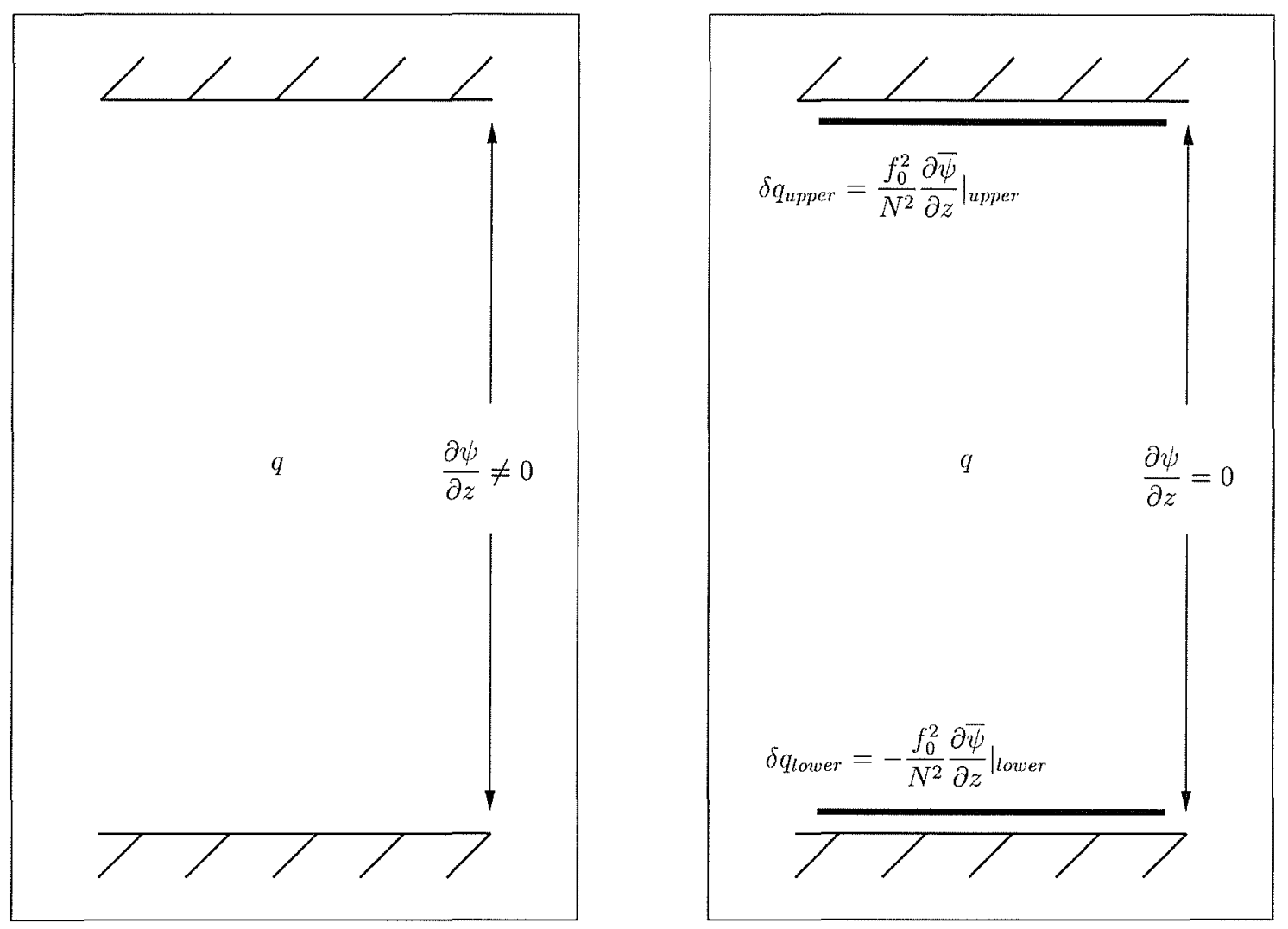

Figure 2.1: Schematic picture of lateral boundary PV sheets. Bretherton (1966) introduced the mathematical equivalence of boundary temperature anomalies to a PV anomaly. It is a powerful technique as it enables us to think of a boundary temperature distribution as part of the PV distribution.

adjacent to the horizontal boundaries. Just inside these boundaries, we place deltafunction sheets of PV, $\delta q_{\text {upper }}$ and $\delta q_{\text {lower }}$, with the size and shape of each representing the magnitude and structure of the temperature distribution on the boundary. Thus:

$$
\tilde{q}=q+\delta q_{\text {upper }}+\delta q_{\text {lower }}
$$

where the delta-function sheets are given by

$$
\delta q_{\text {upper }}=\left.\frac{f_{0}^{2}}{N^{2}} \frac{\partial \bar{\psi}}{\partial z}\right|_{\text {upper }} \quad ; \quad \delta q_{\text {lower }}=-\left.\frac{f_{0}^{2}}{N^{2}} \frac{\partial \bar{\psi}}{\partial z}\right|_{\text {lower }}
$$


The temperature perturbations at the upper and lower boundaries are set to zero, with the actual temperature variation appearing in the PV distribution as delta-function sheets of $\mathrm{PV}$ just interior to the boundary - see figure 2.1 .

\section{Eddy PV fluxes}

We now define a mean streamfunction denoted by an overbar and a fluctuating or eddy part which is the deviation from this average and is denoted by a prime:

$$
\psi=\bar{\psi}+\psi^{\prime}
$$

It follows that the mean quasigeostrophic potential vorticity is

$$
\bar{q}=f_{0}+\beta y+\frac{\partial^{2} \bar{\psi}}{\partial x^{2}}+\frac{\partial^{2} \bar{\psi}}{\partial y^{2}}+f_{0}^{2} \frac{\partial}{\partial z}\left(\frac{1}{N^{2}} \frac{\partial \bar{\psi}}{\partial z}\right)
$$

and the perturbation quasigeostrophic potential vorticity is given by

$$
q^{\prime}=\frac{\partial^{2} \psi^{\prime}}{\partial x^{2}}+\frac{\partial^{2} \psi^{\prime}}{\partial y^{2}}+f_{0}^{2} \frac{\partial}{\partial z}\left(\frac{1}{N^{2}} \frac{\partial \psi^{\prime}}{\partial z}\right)
$$

The geostrophic eddy velocities are given by

$$
u^{\prime}=-\frac{\partial \psi^{\prime}}{\partial y} \quad ; \quad v^{\prime}=\frac{\partial \psi^{\prime}}{\partial x}
$$

with the perturbation temperature given by

$$
T^{\prime}=\frac{f_{0}}{g \alpha} \frac{\partial \psi^{\prime}}{\partial z}
$$

Using equations 2.11 and 2.12 we obtain the un-averaged eddy PV fluxes:

$$
\begin{aligned}
& u^{\prime} q^{\prime}=-\frac{\partial \psi^{\prime}}{\partial y} \frac{\partial^{2} \psi^{\prime}}{\partial x^{2}}-\frac{\partial \psi^{\prime}}{\partial y} \frac{\partial^{2} \psi^{\prime}}{\partial y^{2}}-f_{0}^{2} \frac{\partial \psi^{\prime}}{\partial y} \frac{\partial}{\partial z}\left(\frac{1}{N^{2}} \frac{\partial \psi^{\prime}}{\partial z}\right) \\
& v^{\prime} q^{\prime}=\frac{\partial \psi^{\prime}}{\partial x} \frac{\partial^{2} \psi^{\prime}}{\partial x^{2}}+\frac{\partial \psi^{\prime}}{\partial x} \frac{\partial^{2} \psi^{\prime}}{\partial y^{2}}+f_{0}^{2} \frac{\partial \psi^{\prime}}{\partial x} \frac{\partial}{\partial z}\left(\frac{1}{N^{2}} \frac{\partial \psi^{\prime}}{\partial z}\right)
\end{aligned}
$$


We can evaluate the mean zonal PV flux using the same averaging operator as before. Evaluating the first term in equation 2.14 gives:

$$
\begin{aligned}
\overline{-\frac{\partial \psi^{\prime}}{\partial y} \frac{\partial^{2} \psi^{\prime}}{\partial x^{2}}} & =-\overline{\frac{\partial}{\partial x}\left[\frac{\partial \psi^{\prime}}{\partial y} \frac{\partial \psi^{\prime}}{\partial x}\right]}+\overline{\frac{\partial \psi^{\prime}}{\partial x} \frac{\partial^{2} \psi^{\prime}}{\partial y \partial x}} \\
& =-\overline{\frac{\partial}{\partial x}\left[\frac{\partial \psi^{\prime}}{\partial y} \frac{\partial \psi^{\prime}}{\partial x}\right]}+\overline{\frac{1}{2} \frac{\partial}{\partial y}\left[\left(\frac{\partial \psi^{\prime}}{\partial x}\right)^{2}\right]} \\
& =\frac{\partial}{\partial x}\left(\overline{u^{\prime} v^{\prime}}\right)+\frac{1}{2} \frac{\partial}{\partial y}\left(\overline{v^{\prime 2}}\right)
\end{aligned}
$$

while the second term yields:

$$
\begin{aligned}
-\overline{\frac{\partial \psi^{\prime}}{\partial y} \frac{\partial^{2} \psi^{\prime}}{\partial y^{2}}} & =-\frac{1}{2} \frac{\partial}{\partial y} \overline{\left[\left(\frac{\partial \psi^{\prime}}{\partial y}\right)^{2}\right]} \\
& =-\frac{1}{2} \frac{\partial}{\partial y}\left(\overline{u^{\prime 2}}\right)
\end{aligned}
$$

and the final term in equation 2.14 becomes

$$
\begin{aligned}
-\overline{f_{0}^{2} \frac{\partial \psi^{\prime}}{\partial y} \frac{\partial}{\partial z}\left(\frac{1}{N^{2}} \frac{\partial \psi^{\prime}}{\partial z}\right)} & =-\overline{f_{0}^{2} \frac{\partial}{\partial z}\left[\frac{1}{N^{2}} \frac{\partial \psi^{\prime}}{\partial y} \frac{\partial \psi^{\prime}}{\partial z}\right]}+\overline{\frac{f_{0}^{2}}{N^{2}} \frac{\partial \psi^{\prime}}{\partial z} \frac{\partial^{2} \psi^{\prime}}{\partial y \partial z}} \\
& =-f_{0}^{2} \frac{\partial}{\partial z} \overline{\left[\frac{1}{N^{2}} \frac{\partial \psi^{\prime}}{\partial y} \frac{\partial \psi^{\prime}}{\partial z}\right]}+\frac{f_{0}^{2}}{N^{2}} \frac{\partial}{\partial y}\left(\frac{1}{2} \overline{\left(\frac{\partial \psi^{\prime}}{\partial z}\right)^{2}}\right) \\
& =f_{0}^{2} \frac{\partial}{\partial z}\left(\overline{\frac{u^{\prime} T^{\prime}}{\overline{T_{z}}}}\right)+\frac{g \alpha}{2} \frac{\partial}{\partial y}\left(\overline{\frac{T^{2}}{\overline{T_{z}}}}\right) .
\end{aligned}
$$

Hence the mean zonal eddy PV flux is given by

$$
\overline{u^{\prime} q^{\prime}}=\frac{\partial}{\partial x}\left(\overline{u^{\prime} v^{\prime}}\right)+\frac{1}{2} \frac{\partial}{\partial y}\left(\overline{v^{\prime 2}}\right)-\frac{1}{2} \frac{\partial}{\partial y}\left(\overline{u^{\prime 2}}\right)+f_{0} \frac{\partial}{\partial z}\left(\overline{\frac{u^{\prime} T^{\prime}}{\overline{T_{z}}}}\right)+\frac{g \alpha}{2} \frac{\partial}{\partial y}\left(\overline{\frac{T^{2}}{\overline{T_{z}}}}\right)
$$

The eddy energy density per unit mass is given by

$$
\epsilon=\frac{1}{2}\left(\overline{u^{\prime 2}}+\overline{v^{\prime 2}}+g \alpha \frac{\overline{T^{\prime 2}}}{\overline{T_{z}}}\right)
$$

and so we can rewrite the mean zonal eddy PV flux as

$$
\overline{u^{\prime} q^{\prime}}=\frac{\partial}{\partial x}\left(\overline{u^{\prime} v^{\prime}}\right)+\frac{\partial}{\partial y}\left(\epsilon-\overline{u^{\prime 2}}\right)+f_{0} \frac{\partial}{\partial z}\left(\overline{\frac{u^{\prime} T^{\prime}}{\overline{T_{z}}}}\right) .
$$


Similarly the mean meridional eddy flux of perturbation PV is:

$$
\overline{v^{\prime} q^{\prime}}=\frac{\partial}{\partial x}\left(\overline{v^{\prime 2}}-\epsilon\right)-\frac{\partial}{\partial y}\left(\overline{u^{\prime} v^{\prime}}\right)+f_{0} \frac{\partial}{\partial z}\left(\frac{\overline{v^{\prime} T^{\prime}}}{\overline{T_{z}}}\right) .
$$

Equations 2.21 and 2.22 make clear that the eddy flux of PV - a quantity that is conserved or quasi-conserved during parcel displacement - naturally encapsulates both the momentum and heat transferring properties of quasigeostrophic eddies.

\subsection{The Eulerian mean}

The Eulerian momentum and temperature equations for hydrostatic, Boussinesq flow, subject to forces $\mathbf{F}$ and sources/sinks of heat $G$, are:

$$
\begin{aligned}
\frac{D \mathbf{u}}{D t}+f \mathbf{k} \times \mathbf{u}+\frac{1}{\rho_{0}} \nabla p & =\mathbf{F}, \\
\frac{D T}{D t} & =G,
\end{aligned}
$$

where $\mathbf{u}=(u, v, w)$ is the Eulerian velocity, $f=f(y)$ is the Coriolis parameter, $\rho_{0}$ is a reference value of density, and $D / D t \equiv \partial_{t}+\mathbf{u} \cdot \nabla$ is the total derivative following the Eulerian flow. If it is sensible to define a mean (at present we do not specify whether the averaging procedure is a temporal, spatial, or an ensemble average) then the flow separates into two components: a mean denoted by an overbar $\overline{()}$; and a fluctuating or eddy part which is the deviation from this average:

$$
s^{\prime}=s-\bar{s}
$$

It is assumed that if the mean is a time average, then the eddy statistics are stationary (not changing over time) and if the mean is spatial, then the statistics are homogeneous in one direction or in a limited region. Thus, the sum of the positive deviations from the mean must equal the sum of the negative deviations and so

$$
\overline{s^{\prime}}=0 .
$$


Upon application of the averaging the mean momentum and temperature budgets can be written as:

$$
\begin{aligned}
\frac{D \overline{\mathbf{u}}}{D t}+f \mathbf{k} \times \overline{\mathbf{u}}+\frac{1}{\rho_{0}} \nabla \bar{p} & =\overline{\mathbf{F}}-\frac{1}{2} \nabla \overline{\left(\mathbf{u}^{\prime} \cdot \mathbf{u}^{\prime}\right)}-\overline{\mathbf{u}^{\prime} \times \nabla \times \mathbf{u}^{\prime}}, \\
\frac{D \bar{T}}{D t} & =\bar{G}-\nabla \cdot \overline{\left(\mathbf{u}^{\prime} T^{\prime}\right)}
\end{aligned}
$$

where $D / D t \equiv \partial_{t}+\overline{\mathbf{u}} \cdot \nabla$ is now the total derivative following the Eulerian mean flow. If we consider the zonal and meridional components of equation 2.27 :

$$
\begin{aligned}
& \frac{D \bar{u}}{D t}-f \bar{v}+\frac{1}{\rho_{0}} \partial_{x} \bar{p}=\bar{F}^{x}-\nabla \cdot \overline{\left(\underline{u^{\prime} u^{\prime}}\right)} \\
& \frac{D \bar{v}}{D t}+f \bar{u}+\frac{1}{\rho_{0}} \partial_{y} \bar{p}=\bar{F}_{y}-\nabla \cdot \overline{\left(\underline{u^{\prime} v^{\prime}}\right)}
\end{aligned}
$$

along with equation 2.28 we see that the effect of eddies appears as the divergence of the Reynolds stresses in the momentum equations and the divergence of the eddy heat flux in the temperature equation. This separation of the momentum (vorticity) and heat transporting properties of the eddy field has led to them being treated separately in models. For example, as discussed in chapter 1, Reynolds stresses are almost universally represented as a Fickian process; $\nabla \cdot \overline{\left(\underline{\left.u^{\prime} u^{\prime}\right)}\right.}=-K \nabla^{2} u$ - in large-scale ocean models, even though it is known that quasigeostrophic eddies can, and often do, 'unmix' momentum (Starr, 1948).

Only if these fluxes are represented accurately can the mean flow and tracer distributions be expected to evolve appropriately. Consider a simple thought experiment of zonal flow in thermal wind balance. The eddy terms in equations 2.28 and 2.29 manifest themselves as two terms. However, the eddy temperature fluxes can impact the mean velocities just as the eddy momentum fluxes can, because the flow is in thermal wind balance. For example, if the eddy disturbance is a vertically propagating Rossby wave, its flux characteristics are $\overline{v^{\prime} T^{\prime}} \neq 0$ and $\overline{u^{\prime} v^{\prime}}=0$. First inspection would lead one to believe that the mean zonal momentum is unchanged, because $\overline{u^{\prime} v^{\prime}}=0$. However, the mean state does modify its velocity structure. This is because thermal wind balance 
necessitates a concurrent change in $\bar{u}$. This can only be achieved through a meridional circulation which impacts both heat and momentum budgets. Thus the eddies not only force $D \bar{u} / D t$ and $D \bar{T} / D t$, but also they impact the meridional circulation. This simple thought experiment highlights the fact that one should not consider the eddy momentum flux $\overline{u^{\prime} v^{\prime}}$ and temperature flux $\overline{v^{\prime} T^{\prime}}$ separately. They act in unison, changing the mean flow.

It further highlights a problem associated with the procedure followed in recent oceanographic studies, of the separation of the velocity field into a component (often named the "bolus" velocity) which is the result of the eddies and a component that is independent of them. We see that there is no mean velocity that is autonomous of the eddy disturbances.

A logical way forward is to transform the above equations so that the effect of the eddies on the large-scale appears as an eddy flux of a conserved, and hence more transferable quantity, such as potential vorticity. This can be done by adopting the formalism of the 'transformed Eulerian mean'.

\subsection{The transformed Eulerian mean (TEM)}

The Eulerian mean equations are transformed by introducing a three-dimensional "residual" mean velocity, denoted by $\overline{\mathbf{u}}^{\star}$, which we will insist is nondivergent and is defined thus:

$$
\overline{\mathbf{u}}^{\star}=\overline{\mathbf{u}}+\nabla \times \mathbf{R}
$$

where

$$
\mathbf{R}=\left(\begin{array}{c}
R^{x} \\
R^{y} \\
R^{z}
\end{array}\right)=\left(\begin{array}{c}
-\left(\frac{\overline{v^{\prime} T^{\prime}}}{T_{z}}\right) \\
\left(\frac{\overline{u^{\prime} T^{\prime}}}{T_{z}}\right) \\
\frac{1}{f}\left(\overline{u^{\prime 2}}+\overline{v^{\prime 2}}-\epsilon\right)
\end{array}\right)
$$

can be considered to be the "residual transformation vector" and is chosen such that the appropriate terms appear when the PV equation is formed. $\epsilon$ is the eddy energy density 
per unit mass, defined by equation 2.20 .

The form of the transformed velocity follows from Hoskins (1983) [equations (7.41)] and Plumb (1986) [equations (4.4) and (4.5)] who studied transient eddies in timeaveraged three-dimensional quasigeostrophic flows. The governing equations in those studies were used as diagnostic tools to understand eddy forcing of atmospheric climatologies. As a result the "prognostic" variables in those studies were those of the Eulerian mean flow with the transformed velocities only appearing explicitly in the Coriolis term. The approach here differs because a governing set of equations is sought which is prognostic in the residual mean circulation.

To proceed we use equation 2.31 to substitute for $\overline{\mathbf{u}}$ in terms of $\overline{\mathbf{u}}^{\star}$ in equation 2.27 , which yields:

$$
\begin{aligned}
\frac{D^{\star} \overline{\mathbf{u}^{\star}}}{D t}+f \mathbf{k} \times \overline{\mathbf{u}}^{\star}+\frac{1}{\rho_{0}} \nabla \bar{p}=\overline{\mathbf{F}} & -\frac{1}{2} \nabla \overline{\left(\mathbf{u}^{\prime} \cdot \mathbf{u}^{\prime}\right)}-\overline{\mathbf{u}^{\prime} \times \nabla \times \mathbf{u}^{\prime}} \\
& +f \mathbf{k} \times(\nabla \times \mathbf{R}) \\
& +\frac{D^{\star}}{D t}(\nabla \times \mathbf{R}) \\
& +(\nabla \times \mathbf{R}) \cdot \nabla\left[\overline{\mathbf{u}}^{\star}-(\nabla \times \mathbf{R})\right],
\end{aligned}
$$

where $D^{\star} / D t \equiv \partial_{t}+\overline{\mathbf{u}}^{\star} \cdot \nabla$ is the total derivative following the residual mean flow. Furthermore, 2.28 takes the transformed form:

$$
\frac{D^{\star} \bar{T}}{D t}=\bar{G}-\nabla \cdot \overline{\left(\mathbf{u}^{\prime} T^{\prime}\right)}+(\nabla \times \mathbf{R}) \cdot \nabla \bar{T} .
$$

The left-hand-side of equations 2.33 and 2.34 have the exact form of the mean HPE's but now some of the variables must be reinterpreted (note the ${ }^{*}$ ). At this point it might seem that equations 2.33 and 2.34 are a step backward from equations 2.27 and 2.28 , in that the right-hand-sides of equation 2.33 and 2.34 are much more complicated. But, as I now outline, if the eddy-terms are assumed to obey quasigeostrophic dynamics and scaling, then certain terms on the right-hand-side of equations 2.33 and 2.34 can be justifiably neglected. In effect we are filtering out the eddy terms that we deem are dynamically insignificant. This leads to an elegant simplification, both conceptually and algebraically, to provide insight into the transfer characteristics of the eddy motions. 


\subsection{The TEM in the limit of quasigeostrophic eddies}

\subsubsection{Scaling analysis}

The simplest physical model possessing the essential properties of the eddy transfer is that these fluctuations are near a state of geostrophic and hydrostatic balance. That is, the eddies are quasigeostrophic in nature. With this assumption, certain eddy terms on the right-hand-side of equations 2.33 and 2.34 can be systematically neglected. This yields a set of equations which are simpler to interpret and explore than either equations 2.27 or 2.33 . The scaling assumptions and analysis are now set out in detail.

\section{The momentum equations}

We proceed by focusing on the zonal component of equation 2.33;

$$
\begin{aligned}
\partial_{t} \bar{u}^{\star}+\overline{\mathbf{u}}^{\star} \cdot \nabla \bar{u}^{\star}-f \bar{v}^{\star}+\frac{1}{\rho_{0}} \partial_{x} \bar{p}=\bar{F}^{x} & -\nabla \cdot\left(\overline{\mathbf{u}^{\prime} u^{\prime}}\right) \\
& +[f \mathbf{k} \times(\nabla \times \mathbf{R})]_{x} \\
& +\left[\frac{D^{\star}}{D t}(\nabla \times \mathbf{R})\right]_{x} \\
& +\left[(\nabla \times \mathbf{R}) \cdot \nabla\left[\overline{\mathbf{u}}^{\star}-(\nabla \times \mathbf{R})\right]\right]_{x}
\end{aligned}
$$

and then we will extend the results to the meridional and vertical components.

The properties of the flow have associated scales which are chosen as follows: 


$\begin{array}{lll}\text { Symbol } & \text { Property } & \text { Scale } \\ & & \\ \left(\bar{u}^{\star}, \bar{v}^{\star}\right) & \text { mean horizontal velocities } & \mathbf{U} \\ \bar{w}^{\star} & \text { mean vertical velocity } & \mathbf{W} \\ \bar{p} & \text { mean pressure } & \mathbf{P} \\ & \text { mean horizontal length scale } & \mathbf{L} \\ & \text { mean vertical length scale } & \mathbf{H} \\ \bar{T} & \text { mean temperature } & \mathbf{T} \\ N^{2} & \text { mean stratification } & \mathbf{N} \\ f & \text { Coriolis parameter } & \mathbf{f} \\ \left(u^{\prime}, v^{\prime}\right) & \text { perturbation horizontal velocities } & \mathbf{U}^{\prime} \\ w^{\prime} & \text { perturbation vertical velocity } & - \\ & \text { perturbation length scale } & \mathbf{L}_{\mathbf{d}} \\ T^{\prime} & \text { perturbation temperature } & \mathbf{T}^{\prime}\end{array}$

If the large scale flow is in approximate geostrophic balance then we obtain a scale for $\mathbf{P}$ in terms of $\mathbf{U}, \mathbf{L}$ and $f$. Since

$$
-f \bar{v}^{\star}=\frac{1}{\rho_{0}} \partial_{x} \bar{p}
$$

then

$$
\mathrm{fU} \sim \frac{1}{\rho} \frac{\mathbf{P}}{\mathbf{L}}
$$

thus

$$
\mathbf{P} \sim \rho \mathbf{f U L}
$$

where the symbol " $\sim$ " denotes order of magnitude equality. Quasigeostrophic eddies are close to being in thermal wind balance. This fact allows us to obtain a relationship between the velocity and temperature properties of the eddies. Thermal wind relates the vertical velocity shear to the lateral temperature gradients;

$$
f \partial_{z} v^{\prime}=g \alpha \partial_{y} T^{\prime}
$$


thus

$$
\mathbf{f} \frac{\mathrm{U}^{\prime}}{\mathbf{H}} \sim g \alpha \frac{\mathrm{T}^{\prime}}{\mathrm{L}_{\mathbf{d}}},
$$

and so

$$
\mathbf{T}^{\prime} \sim \frac{\mathbf{f U}^{\prime} \mathbf{L}_{\mathrm{d}}}{g \alpha \mathbf{H}} .
$$

We now systematically determine the order of magnitude of the components of the vector $\mathbf{R}$, whose curl is the difference between the Eulerian and residual mean velocities. The zonal component of $\mathbf{R}$ is

$$
R^{x}=-\left(\frac{\overline{v^{\prime} T^{\prime}}}{T_{z}}\right),
$$

which scales as

$$
\begin{aligned}
\mathbf{R}^{\mathbf{x}} & \sim \frac{\mathrm{U}^{\prime} \mathrm{T}^{\prime} \mathbf{H}}{\mathbf{T}} \\
& \sim \frac{\mathrm{fU}^{\prime 2} \mathbf{L}_{\mathrm{d}}}{g \alpha \mathrm{T}} \\
& \sim \frac{\mathrm{fU}^{\prime 2} \mathbf{L}_{\mathrm{d}}}{\mathrm{N}^{2} \mathrm{H}} \\
& \sim \frac{\mathrm{U}^{\prime 2} \mathrm{H}}{\mathrm{fL}_{\mathrm{d}}}\left(\frac{\mathrm{L}_{\mathrm{d}} \mathbf{f}}{\mathrm{NH}}\right)^{2} .
\end{aligned}
$$

Now the deformation radius $L_{d}$ scales as:

$$
\mathrm{L}_{\mathrm{d}} \sim \frac{\mathrm{NH}}{\mathrm{f}},
$$

therefore

$$
\mathbf{R}^{\mathrm{x}} \sim \frac{\mathrm{U}^{\prime 2} \mathrm{H}}{\mathrm{fL}_{\mathrm{d}}} .
$$

Similarly, since

$$
R^{y}=\left(\frac{\overline{u^{\prime} T^{\prime}}}{T_{z}}\right)
$$

then $R^{y}$ scales as

$$
\mathrm{R}^{\mathrm{y}} \sim \frac{\mathrm{U}^{\prime 2} \mathrm{H}}{\mathrm{fL}_{\mathrm{d}}} .
$$

The vertical component of $\mathbf{R}$ is related to the eddy energy density, viz:

$$
R^{z}=\frac{1}{f}\left(\overline{u^{\prime 2}}+\overline{v^{\prime 2}}-\epsilon\right) .
$$


Because the eddies manifest themselves on the scale of the deformation radius, the eddy kinetic and potential energies are of comparable magnitude. Thus $R^{z}$ scales as

$$
\mathrm{R}^{\mathrm{z}} \sim \frac{\mathrm{U}^{\prime 2}}{\mathrm{f}} .
$$

We now write

$$
\nabla \times \mathbf{R}=\left(\begin{array}{c}
C^{x} \\
C^{y} \\
C^{z}
\end{array}\right)=\left(\begin{array}{c}
\partial_{y} R^{z}-\partial_{z} R^{y} \\
\partial_{z} R^{x}-\partial_{x} R^{z} \\
\partial_{x} R^{y}-\partial_{y} R^{x}
\end{array}\right)
$$

Hence

$$
\mathbf{k} \times(\nabla \times \mathbf{R})=\left(\begin{array}{c}
-C^{y} \\
C^{x} \\
0
\end{array}\right) .
$$

With this notation equation 2.35 can be rewritten as:

$$
\partial_{t} \bar{u}^{\star}+\overline{\mathbf{u}}^{\star} \cdot \nabla \bar{u}^{\star}-f \bar{v}^{\star}+\frac{1}{\rho_{0}} \partial_{x} \bar{p}=\bar{F}^{x}-\nabla \cdot\left(\overline{\mathbf{u}^{\prime} u^{\prime}}\right)+f C^{y}+\overline{\mathbf{u}}^{\star} \partial_{x} C^{x}+C^{x} \partial_{x} \bar{u} .
$$

Scaling for $C^{x}$ :

$$
\begin{aligned}
\mathrm{C}^{\mathbf{x}} & \sim\left(\frac{\mathrm{R}^{\mathbf{z}}}{\mathrm{L}}, \frac{\mathrm{R}^{\mathbf{y}}}{\mathrm{H}}\right), \\
& \sim\left(\frac{\mathrm{U}^{\prime 2}}{\mathrm{fL}}, \frac{\mathrm{U}^{\prime 2}}{\mathrm{fL}_{\mathrm{d}}}\right) .
\end{aligned}
$$

Similarly for $C^{y}$ :

$$
\begin{aligned}
\mathrm{C}^{\mathrm{y}} & \sim\left(\frac{\mathrm{R}^{\mathrm{x}}}{\mathrm{H}}, \frac{\mathrm{R}^{\mathrm{z}}}{\mathrm{L}}\right) \\
& \sim\left(\frac{\mathrm{U}^{\prime 2}}{\mathrm{fL}_{\mathrm{d}}}, \frac{\mathrm{U}^{\prime 2}}{\mathrm{fL}}\right) .
\end{aligned}
$$

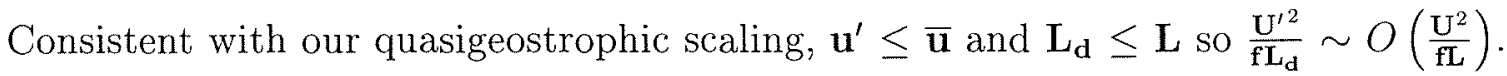

We are now in a position to scale each term in equation 2.44 . 


\begin{tabular}{|c|c|c|c|c|c|c|c|c|}
\hline$\partial_{t} \bar{u}^{\star}$ & $\overline{\mathbf{u}}^{\star} \cdot \nabla \bar{u}^{\star}$ & $-f \bar{v}^{\star}$ & $\frac{1}{\rho_{0}} \partial_{x} \bar{p}$ & $\bar{F}^{x}$ & $-\nabla \cdot\left(\overline{u^{\prime} u^{\prime}}\right)$ & $f C^{y}$ & $\overline{\mathbf{u}}^{\star} \partial_{x} C^{x}$ & $C^{x} \partial_{x} \bar{u}$ \\
\hline$\downarrow$ & $\downarrow$ & $\downarrow$ & $\downarrow$ & $\downarrow$ & $\downarrow$ & $\downarrow$ & $\downarrow$ & $\downarrow$ \\
\hline$\frac{\mathrm{U}}{\tau}$ & $\frac{\mathrm{U}^{2}}{\mathrm{~L}}$ & $\mathrm{fU}$ & $\frac{p}{\rho_{0} \mathrm{~L}}$ & $\mathrm{~F}$ & $\frac{U^{2}}{L}$ & $\mathrm{fC}^{\mathrm{y}}$ & $\frac{U C^{x}}{L}$ & $\frac{\mathrm{UC}^{\mathrm{x}}}{\mathrm{L}}$ \\
\hline$\frac{1}{f \tau}$ & $\frac{\mathrm{U}}{\mathrm{fL}}$ & 1 & 1 & $\frac{F}{f U}$ & $\frac{\mathrm{U}}{\mathrm{fL}}$ & $\frac{\mathrm{C}^{\mathrm{y}}}{\mathrm{U}}$ & $\frac{\mathrm{C}^{\mathrm{x}}}{\mathrm{fL}}$ & $\frac{C^{x}}{f L}$ \\
\hline$\frac{1}{\mathbf{f} \tau}$ & $\frac{\mathrm{U}}{\mathrm{fL}}$ & 1 & 1 & $\frac{F}{f U}$ & $\frac{\mathrm{U}}{\mathrm{fL}}$ & $\frac{\mathrm{U}}{\mathrm{fL}}$ & $\left(\frac{\mathrm{U}}{\mathrm{fL}}\right)^{2}$ & $\left(\frac{\mathrm{U}}{\mathrm{fL}}\right)^{2}$ \\
\hline
\end{tabular}

The non-dimensional number $R_{o}=\frac{U}{f L}$ is the Rossby number. For all flows studied in this thesis $R_{o} \ll 1$. Neglecting terms that are smaller than $O\left(R_{o}\right)$ allows us to neglect the last two terms on the right-hand-side of equation 2.44. Thus the eddy temperature flux enters the mean momentum budget through a Coriolis torque. Through neglect of the $O\left(R_{o}^{2}\right)$ terms and evaluation of the $f C^{y}$ term we have:

$$
\frac{D^{\star} \bar{u}^{\star}}{D t}-f \bar{v}^{\star}+\frac{1}{\rho_{0}} \partial_{x} \bar{p}=\bar{F}^{x}+\partial_{x}\left(\overline{v^{\prime 2}}-\epsilon\right)-\partial_{y}\left(\overline{u^{\prime} v^{\prime}}\right)+f \partial_{z}\left(\overline{\frac{v^{\prime} T^{\prime}}{\overline{T_{z}}}}\right) .
$$

Now, as defined in equation 2.21

$$
\overline{v^{\prime} q^{\prime}}=\frac{\partial}{\partial x}\left(\overline{v^{\prime 2}}-\epsilon\right)-\frac{\partial}{\partial y}\left(\overline{u^{\prime} v^{\prime}}\right)+f_{0} \frac{\partial}{\partial z}\left(\overline{\frac{v^{\prime} T^{\prime}}{\overline{T_{z}}}}\right)
$$

thus the last three terms in equation 2.47 can be replaced by the eddy PV flux to yield

$$
\frac{D^{\star} \bar{u}^{\star}}{D t}-f \bar{v}^{\star}+\frac{1}{\rho_{0}} \partial_{x} \bar{p}=\bar{F}^{x}+\overline{v^{\prime} q^{\prime}}
$$

Following the same scaling procedure and using equation 2.22 , the meridional momentum equation reduces to:

$$
\frac{D^{\star} \bar{v}^{\star}}{D t}+f \bar{u}^{\star}+\frac{1}{\rho_{0}} \partial_{y} \bar{p}=\bar{F}^{y}-\overline{u^{\prime} q^{\prime}}
$$

Hence in the limit of quasigeostrophic eddies equation 2.33 simplifies to

$$
\frac{D^{\star} \overline{\mathbf{u}}^{\star}}{D t}+f \mathbf{k} \times \overline{\mathbf{u}}^{\star}+\frac{1}{\rho_{0}} \nabla \bar{p}=\overline{\mathbf{F}}-\mathbf{k} \times \overline{\left(\mathbf{u}^{\prime} q^{\prime}\right)} .
$$




\section{The temperature equation}

Using the same notation, equation 2.34 can be written as

$$
\frac{D^{\star} \bar{T}}{D t}=\bar{G}-\nabla \cdot \overline{\left(\mathbf{u}^{\prime} T^{\prime}\right)}+C^{x} \partial_{x} \bar{T}+C^{y} \partial_{y} \bar{T}+C^{z} \partial_{z} \bar{T} .
$$

Now,

$$
\begin{aligned}
C^{z} \partial_{z} \bar{T} & =\left(\partial_{x} R^{y}-\partial_{y} R^{x}\right) \partial_{z} \bar{T} \\
& =\left(\partial_{x}\left(\frac{\overline{u^{\prime} T^{\prime}}}{\overline{T_{z}}}\right)+\partial_{y}\left(\frac{\overline{v^{\prime} T^{\prime}}}{\bar{T}_{z}}\right)\right) \partial_{z} \bar{T} \\
& =\partial_{x}\left(\overline{u^{\prime} T^{\prime}}\right)+\partial_{y}\left(\overline{v^{\prime} T^{\prime}}\right) \\
& =\nabla_{h} \cdot \overline{\left(\mathbf{u}^{\prime} T^{\prime}\right)}
\end{aligned}
$$

with the quasigeostrophic assumption that $\bar{T}_{z}=\bar{T}_{z}(z)$ and $\nabla_{h}=\partial_{x}+\partial_{y}$. Thus with equation 2.52 , equation 2.51 becomes

$$
\frac{D^{\star} \bar{T}}{D t}=\bar{G}-\partial_{z} \overline{\left(w^{\prime} T^{\prime}\right)}+C^{x} \partial_{x} \bar{T}+C^{y} \partial_{y} \bar{T} .
$$

We now systematically scale each term in equation 2.53 :

$\begin{array}{cccccc}\partial_{t} \bar{T} & \overline{\mathbf{u}}^{\star} \cdot \nabla \bar{T} & \bar{G} & -\partial_{z} \overline{\left(w^{\prime} T^{\prime}\right)} & C^{x} \partial_{x} \bar{T} & C^{y} \partial_{y} \bar{T} \\ \downarrow & \downarrow & \downarrow & \downarrow & \downarrow & \downarrow \\ \frac{T}{\tau} & \frac{\mathrm{UT}}{\mathrm{L}} & \mathbf{G} & 0 & \frac{\mathrm{C}^{\mathbf{T}}}{\mathrm{L}} & \frac{\mathrm{C}^{\mathbf{y} T}}{\mathrm{~L}} \\ \frac{\mathrm{T}}{\tau} & \frac{\mathrm{UT}}{\mathrm{L}} & \mathbf{G} & 0 & \left(\frac{\mathrm{U}}{\mathrm{fL}}\right) \frac{\mathrm{UT}}{\mathrm{L}} & \left(\frac{\mathrm{U}}{\mathrm{fL}}\right) \frac{\mathrm{UT}}{\mathrm{L}}\end{array}$

The last two terms are $O\left(R_{o}\right)$ smaller than the substantial derivative terms and so to be consistent with the procedure employed for the momentum equations, we neglect them. The $\overline{\left(w^{\prime} T^{\prime}\right)}$ vanishes for quasigeostrophic eddies. 
Thus the transformed temperature equation can be written as

$$
\frac{D^{\star} \bar{T}}{D t}=\bar{G},
$$

and our governing equations have become beautifully simple:

$$
\begin{aligned}
\frac{D^{\star} \overline{\mathbf{u}}^{\star}}{D t}+f \mathbf{k} \times \overline{\mathbf{u}}^{\star}+\frac{1}{\rho_{0}} \nabla \bar{p} & =\overline{\mathbf{F}}-\mathbf{k} \times \overline{\left(\mathbf{u}^{\prime} q^{\prime}\right)} \\
\frac{D^{\star} \bar{T}}{D t} & =\bar{G}
\end{aligned}
$$

Equation set 2.55.a-b are primitive equations for the mean flow in which the eddies obey quasigeostrophic dynamics. Using primitive equations for the mean flow and quasigeostrophic dynamics for the eddy motions will not be appropriate for all circumstances. However for large-scale ocean climate models, the Rossby numbers of the eddy motions and large-scale flow are appropriately small such that the quasigeostrophic framework is appropriate for the eddies.

\subsection{Representation of eddies by a PV flux}

The governing equations have been transformed and an assumption has been made regarding the dynamics of the eddies, which leads to one term that represents the effect of the eddies on the mean flow. As equations 2.55.a-b show, this term acts as an effective body force in the momentum equations; $\overline{\mathbf{F}}_{\text {eddies }}=-\mathbf{k} \times \overline{\left(\mathbf{u}^{\prime} q^{\prime}\right)}$.

These equations differ from the TEM approaches offered previously in the atmospheric literature [Andrews and McIntyre (1976), Edmon et al. (1980)] and recently in the ocean context [Lee and Leach (1996) and Greatbach (1998)]. This is due the fact that equations 2.55.a-b are prognostic in the transformed (starred) velocities and nowhere does the mean

velocity, $\overline{\mathbf{u}}$, appear. This is a tremendous advantage over governing equations in which a mixture of the Eulerian mean and residual mean velocities appear, because the residual 
mean velocities do not have to be diagnosed. In this approach, the transformed velocities are determined implicitly.

With the model velocities being those of the residual mean circulation, $\overline{\mathbf{u}}^{\star}$, the issue arises of how to make comparisons to oceanic observations of $\overline{\mathbf{u}}$. However, the reason for wanting to compare the velocity fields is unclear to me. For climate questions, what should be compared are the distributions of tracer and water properties. Information obtained through direct observations give instantaneous water mass and velocity distributions, which when averaged over a period of time are reported to give the Eulerian mean fields. However, as discussed in chapter 1, the mean flow is forced and modified by eddy motions (momentum transport) and heat and tracers are transferred by the eddies, modifying water mass distributions. When thinking about the mean climatic distributions of tracer and water masses and their transport, it is not the Eulerian mean velocities that are appropriate, but instead it those of the residual mean. So to make a direct comparison to observations, the residual mean velocities ( $\overline{\mathbf{u}}^{\star}$ 's) would have to diagnosed for the observed fields. The eddy fluxes (and their derivatives) in the real ocean are needed to do this, and this is a difficult task indeed. What should not be done is a comparison of Eulerian mean fields with those of the transformed Eulerian mean since they can look very different, particularly in the meridional plane. For example, in studies of the troposphere the multiple cells observed in the Eulerian mean (Hadley, Ferrel) vanish in the TEM, resulting in a single thermally direct overturning cell.

If eddy-resolving and parameterized models are being compared, the task is easier because the $\overline{\mathbf{u}^{\star}}$ 's can be diagnosed using the resolved eddy fluxes. It can be justified that this comparison should be done as it is a direct test of the theoretical framework argued in this chapter. However, with regard to the real ocean and atmosphere, the Eulerian mean velocity $(\overline{\mathbf{u}})$ is only part of the story of the transfer of fluid and its tracer properties and so is not needed by itself.

Plumb (1986) shows that each component of the PV flux can be written as the divergence of another flux, viz. 


$$
\overline{\mathbf{u}^{\prime} q^{\prime}}=\partial_{i} B_{i j}
$$

where $\partial_{i} \equiv\left(\partial_{1}, \partial_{2}, \partial_{3}\right)=\left(\partial_{x}, \partial_{y}, \partial_{z}\right)$, and

$$
\mathbf{B}=\left(\begin{array}{ccc}
\overline{u^{\prime} v^{\prime}} & \epsilon-\overline{u^{\prime 2}} & f\left(\frac{\overline{u^{\prime} T^{\prime}}}{\overline{T_{z}}}\right) \\
\overline{v^{\prime 2}}-\epsilon & -\overline{u^{\prime} v^{\prime}} & f\left(\frac{\overline{u^{\prime} T^{\prime}}}{\bar{T}_{z}}\right) \\
0 & 0 & 0
\end{array}\right)
$$

This is the equivalent for time-mean flows to the practice of writing the forcing in a zonalmean flow as the divergence of the Eliassen-Palm flux as outlined in section 2.2.1. As will become apparent later in the thesis, there is considerable advantage in working with the Eliassen-Palm flux in zonal-flows. This flux is particularly illuminating when considering momentum constraints for the zonal-mean flow (introduced later in section 2.8.1). This is because the volume integral of the Eliassen-Palm flux divergence vanishes in the zonal case. This gives a constraint for any parameterization used to close for the PV flux. For time-mean flows the advantage is less transparent because the volume integral of each component of the PV flux does not necessarily vanish for free slip boundary conditions on the velocity field. For no-slip boundary conditions, the volume integral of the PV flux does vanish for the time mean flow. For free-slip conditions, consider the zonal component of the PV flux in a domain bounded in $x$ between $x=x_{1}$ and $x=x_{2}$, in $y$ between $y=y_{1}$ and $y=y_{2}$, and vertically between the planes $z=z_{1}$ and $z=z_{2}$. Since

$$
\overline{u^{\prime} q^{\prime}}=\frac{\partial}{\partial x}\left(\overline{u^{\prime} v^{\prime}}\right)+\frac{\partial}{\partial y}\left(\epsilon-\overline{u^{\prime 2}}\right)+f_{0} \frac{\partial}{\partial z}\left(\overline{\frac{u^{\prime} T^{\prime}}{\overline{T_{z}}}}\right)
$$

it follows that

$$
\begin{aligned}
\int_{V o l} \overline{u^{\prime} q^{\prime}} d V= & \int_{y_{1}}^{y_{2}} \int_{z_{1}}^{z_{2}}\left[\overline{u^{\prime} v^{\prime}}\right]_{x_{1}}^{x_{2}} d y d z \\
& +\int_{x_{1}}^{x_{2}} \int_{z_{1}}^{z_{2}}\left[\epsilon-\overline{u^{\prime 2}}\right]_{y_{1}}^{y_{2}} d x d z \\
& +\int_{x_{1}}^{x_{2}} \int_{y_{1}}^{y_{2}}\left[f_{0}\left(\frac{\overline{u^{\prime} T^{\prime}}}{\overline{T_{z}}}\right)\right]_{z_{1}}^{z_{2}} d x d y
\end{aligned}
$$


The first term on the right-hand-side is zero because $u^{\prime}=0$ at $x=x_{1}$ and $x=x_{2}$. The third term is also zero because $T^{\prime}=0$ at $z=z_{1}$ and $z=z_{2}$ due to the use of the PV sheets. However, the second term does not vanish and yields

$$
\int_{V o l} \overline{u^{\prime} q^{\prime}} d V=\int_{x_{1}}^{x_{2}} \int_{z_{1}}^{z_{2}}\left[\frac{1}{2}\left(-\overline{u^{\prime 2}}+g \alpha \overline{T^{\prime 2}} / \overline{T_{z}}\right)\right]_{y_{1}}^{y_{2}} d x d z
$$

Similarly,

$$
\int_{V o l} \overline{v^{\prime} q^{\prime}} d V=\int_{y_{1}}^{y_{2}} \int_{z_{1}}^{z_{2}}\left[\frac{1}{2}\left(\overline{v^{\prime 2}}-g \alpha \overline{T^{\prime 2}} / \overline{T_{z}}\right)\right]_{x_{1}}^{x_{2}} d y d z
$$

Thus for general mean flows with free-slip boundary condition on velocity, the global integral of the PV flux does not vanish because of boundary flux contributions. These boundary contributions arise because they act to balance the volume integral of the Coriolis term $f \mathbf{k} \times \overline{\mathbf{u}}^{\star}$, which does not vanish under free-slip boundary conditions.

\subsection{The equations for time mean flows}

We now interpret the averaging procedure for time mean flows. The equations can be readily obtained from equations 2.55 .a-.b, and are restated:

$$
\begin{aligned}
\frac{D^{\star} \bar{u}^{\star}}{D t}-f \bar{v}^{\star}+\frac{1}{\rho_{0}} \partial_{x} \bar{p} & =\bar{F}_{x}+\overline{v^{\prime} q^{\prime}} \\
\frac{D^{\star} \bar{v}^{\star}}{D t}+f \bar{u}^{\star}+\frac{1}{\rho_{0}} \partial_{y} \bar{p} & =\bar{F}_{y}-\overline{u^{\prime} q^{\prime}} \\
\rho_{0} g \alpha \bar{T}+\partial_{z} \bar{p} & =0, \\
\frac{D^{\star} \bar{T}}{D t} & =\bar{G} \\
\nabla \cdot \overline{\mathbf{u}}^{\star} & =0 .
\end{aligned}
$$

where $D^{\star} / D t \equiv \overline{\mathbf{u}}^{\star} \cdot \nabla$.

There are five equations (2.60.a-e) and seven unknowns; $\bar{u}, \bar{v}^{\star}, \bar{w}^{\star}, \bar{T}, \bar{p}, \overline{u^{\prime} q^{\prime}}$, and $\overline{v^{\prime} q^{\prime}}$. If the eddy PV fluxes can be expressed in terms of variables on the left-hand-side 
of equations 2.60.a-e, then a closed set of equations for the time mean flow are obtained in which the eddies appear as a body force in the momentum equations.

\subsection{The equations for zonal mean flows}

We now consider flows which are primarily in the zonal direction, such as the jet stream in the atmosphere or the Antarctic Circumpolar Current in the ocean. From equations 2.31 and 2.32 the transformed velocities appear thus:

$$
\begin{aligned}
\bar{u}^{\star} & =\bar{u}+\left(\partial_{y} R^{z}-\partial_{z} R^{y}\right), \\
\bar{v}^{\star} & =\bar{v}+\partial_{z} R^{x}, \\
\bar{w}^{\star} & =\bar{w}-\partial_{y} R^{x}
\end{aligned}
$$

since for zonal mean flows $\partial_{x} \equiv 0$. If we scale 2.61.a:

$$
\begin{array}{ccc}
\bar{u}^{\star} & \bar{u} & C^{x} \\
\downarrow & \downarrow & \downarrow \\
& & \\
\mathrm{U} & \mathbf{U} & \mathrm{U} \frac{\mathbf{U}}{\mathrm{fL}}
\end{array}
$$

The term which redefines the zonal velocity is $O\left(R_{o}\right)$ smaller than the zonal mean zonal velocity and, if worked through, is on the same order as the terms neglected, through the assumption of quasigeostrophic eddies, in section 2.5. For this reason, when working in the zonal mean, the zonal mean zonal velocity is not transformed. Only the velocities in the meridional plane are altered. Hence it is consistent with our scaling assumptions to let $\mathbf{R}$ take the form

$$
\mathbf{R}=\left(\begin{array}{c}
R^{x} \\
R^{y} \\
R^{z}
\end{array}\right)=\left(\begin{array}{c}
-\left(\overline{\frac{\bar{y}^{\prime} T^{\prime}}{T_{z}}}\right) \\
0 \\
0
\end{array}\right)
$$


to give the "residual mean meridional circulation", viz:

$$
\begin{aligned}
\bar{v}^{\star} & =\bar{v}-\partial_{z}\left(\frac{\overline{v^{\prime} T^{\prime}}}{\overline{T_{z}}}\right), \\
\bar{w}^{\star} & =\bar{w}+\partial_{y}\left(\frac{\overline{v^{\prime} T^{\prime}}}{\bar{T}_{z}}\right) .
\end{aligned}
$$

With the zonal mean $\mathbf{R}$ defined in equation 2.62 , the governing equations become:

$$
\begin{aligned}
\frac{D^{\star} \bar{u}}{D t}-f \bar{v}^{\star} & =\bar{F}^{x}+\overline{v^{\prime} q^{\prime}} \\
\frac{D^{\star} \bar{v}^{\star}}{D t}+f \bar{u}+\frac{1}{\rho_{0}} \partial_{y} \bar{p} & =\bar{F}^{y} \\
\rho_{0} g \alpha \bar{T}+\partial_{z} \bar{p} & =0 \\
\frac{D^{\star} \bar{T}}{D t}=\bar{G} & \\
\partial_{y} \bar{v}^{\star}+\partial_{z} \bar{w}^{\star} & =0
\end{aligned}
$$

where $D^{\star} / D t \equiv \partial_{t}+\bar{v}^{\star} \partial_{y}+\bar{w}^{\star} \partial_{z}$. There are five equations (2.64.a-e) and six unknowns; $\bar{u}, \bar{v}^{\star}, \bar{w}^{\star}, \bar{T}, \bar{p}$, and $\overline{v^{\prime} q^{\prime}}$. If the eddy term $\overline{v^{\prime} q^{\prime}}$ can be expressed in terms of variables on the left-hand-side of equation 3.27, then a closed set of prognostic equations for the zonal mean flow are obtained in which the eddies appear as a single body force in the zonal momentum equation.

\subsubsection{Eddy-propagation, transport and integral constraints}

The only eddy forcing term that remains in the zonal mean formalism is the divergence of the quasigeostrophic PV flux which under quasigeostrophic scaling is identically equal to the divergence of the Eliassen-Palm flux E:

$$
\overline{v^{\prime} q^{\prime}}=\nabla \cdot \mathbf{E}
$$

$\mathrm{E}$ is given by

$$
\mathrm{E}=\left(\begin{array}{c}
E_{y} \\
E_{z}
\end{array}\right)=\left(\begin{array}{c}
-\overline{\left(u^{\prime} v^{\prime}\right)} \\
f_{o}\left(\frac{\overline{v^{\prime} \rho^{\prime}}}{\overline{\rho_{z}}}\right)
\end{array}\right),
$$


where $f_{o}$ is a middle-latitude value of the Coriolis parameter.

Under quasigeostrophic scaling the effect of eddies in the zonal average appears as a body force equal to the divergence of the Eliassen-Palm flux. Andrews and McIntyre $(1978 \mathrm{a}, \mathrm{b})$ showed that this flux depends on properties of the eddy disturbances. Using quasigeostrophic theory they introduced the generalized Eliassen-Palm relation for smallamplitude wave-like eddies;

$$
\frac{\partial A}{\partial t}+\nabla \cdot \mathbf{E}=\mathcal{D}
$$

Here $A$ is the zonally-averaged wave activity;

$$
A=\frac{1}{2} \frac{\overline{q^{\prime 2}}}{\bar{q}_{y}}
$$

and $\mathcal{D}$ represents non-conservative eddy effects;

$$
\mathcal{D}=\frac{\overline{q^{\prime} \chi^{\prime}}}{\bar{q}_{y}},
$$

where $\chi^{\prime}$ is the source and sinks of potential vorticity originating from frictional and/or diabatic effects given by

$$
\chi^{\prime}=\frac{\partial F_{y}^{\prime}}{\partial x}-\frac{\partial F_{x}^{\prime}}{\partial y}+\frac{\partial}{\partial z}\left(\frac{G^{\prime}}{\bar{\rho}_{z}}\right) .
$$

Equation (2.67) makes explicit the dependence of $\nabla \cdot \mathbf{E}$ on the physics of wave transience and non-conservative effects. If the waves are conservative, $\mathcal{D}=0$, then $A$ must increase in regions where $\mathbf{E}$ converges and decrease where $\mathbf{E}$ is divergent. $\mathbf{E}$ is therefore a useful measure of the propagation of wave activity. If the eddies are conservative and their amplitude does not change with time, then $\mathbf{E}$ is non-divergent, $\overline{v^{\prime} q^{\prime}}=0$ and eddies do not change mean properties. This is the non-acceleration theorem, first noted by Charney and Drazin (1961). Such a result is clear from (2.64.a), but not obvious from the Eulerian mean equations (2.29-e). In the latter case, the separate eddy forcing terms do not necessarily vanish even though $\nabla \cdot \mathrm{E}=0$, but instead modify the Eulerian mean circulation $(\bar{v}, \bar{w})$ to cancel the non-zero eddy terms. Thus we see that couching eddy terms as EP fluxes provides a useful measure of propagation of eddy wave activity and a clear framework to determine the effect of the eddies on the mean flow. Contours of $\nabla \cdot \mathbf{E}$ 
show the zonal force per unit mass exerted by the quasigeostrophic eddies and yield the net, non-trivial, effect of eddies on mean flow.

The Eliassen-Palm flux is also useful when thinking about the role of boundaries, particularly when used in conjunction with the PV sheets introduced in section 2.2.2. Thus, if the vertical component of $\mathbf{E}$ (that connected with eddy heat flux) is finite at an infinitesimal distance from the boundary, it is zero on the boundary itself in the presence of the PV sheet. This leads to a concentrated sheet of $\nabla \cdot \mathbf{E}$ representing PV fluxes associated with boundary temperature distributions. Figure 2.2 shows idealized schematic diagrams of the most unstable Eady and Charney modes. For the Eady mode (figure 2.2.a) $\mathrm{E}$ is independent of height and is therefore non-divergent in the interior. Thus concentrated regions of $\mathrm{E}$ divergence (PV sheets) are present at the upper and lower boundaries. Temperature perturbations at the lower boundary give rise to a divergent sheet and a compensating convergent sheet at the upper boundary. If the sheets differ in spatial structure, as in figure 2.2 where the lower sheet is of less meridional extent than that of the upper sheet, then there has to be a non-zero meridional component of E. As explained earlier this component, $E_{y}$ is equal in size and opposite in direction to the momentum flux, as can be seen through (2.66).

The picture for the Charney mode (figure 2.2.b) is somewhat different. Again, the temperature perturbations at the lower boundary give divergence, while the compensating convergence occurs in the interior around the steering level. In both cases the divergent lower boundary is active in the baroclinic instability process and the eddy forcing of the mean flow.

In the presence of PV sheets there is an important and very useful integral constraint on $\underline{\nabla} \cdot \mathrm{E}$ :

$$
\int_{V o l u m e} \underline{\nabla} \cdot \mathbf{E} d V=0
$$

where the volume considered includes the sheets. Thus the eddies can provide no net force on the zonal mean flow, acting only to redistribute momentum within the flow. This momentum constraint will be exploited in chapter 3 , to constrain our choice of the 
(a)

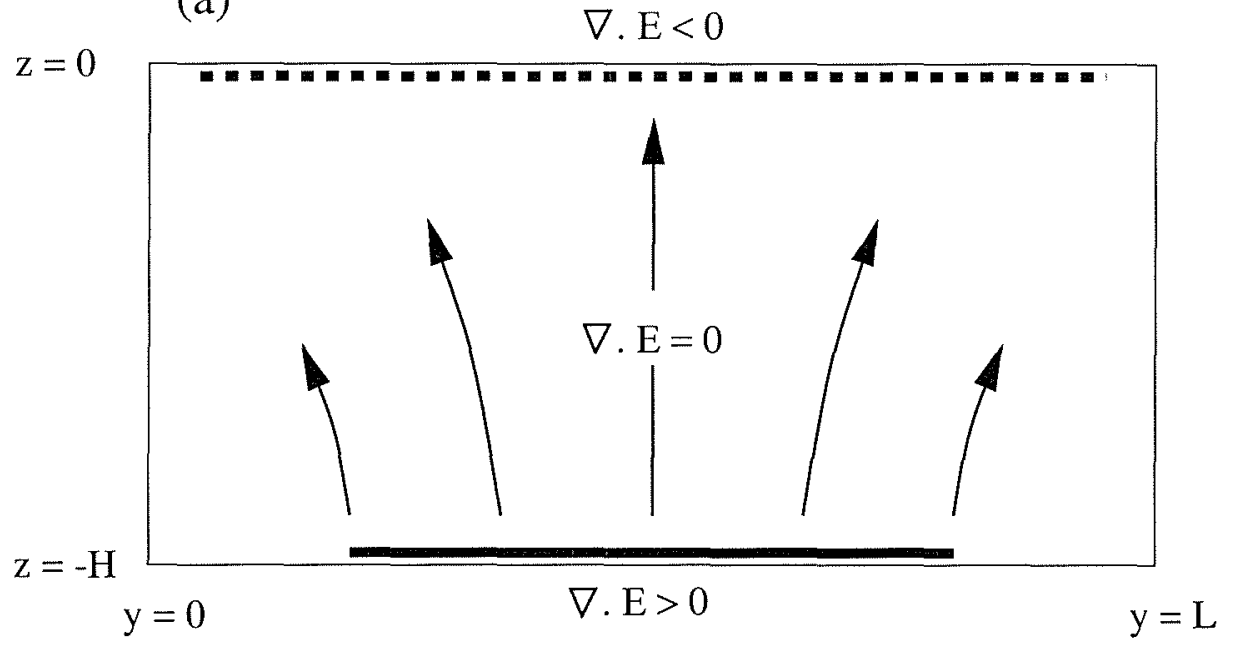

(b)

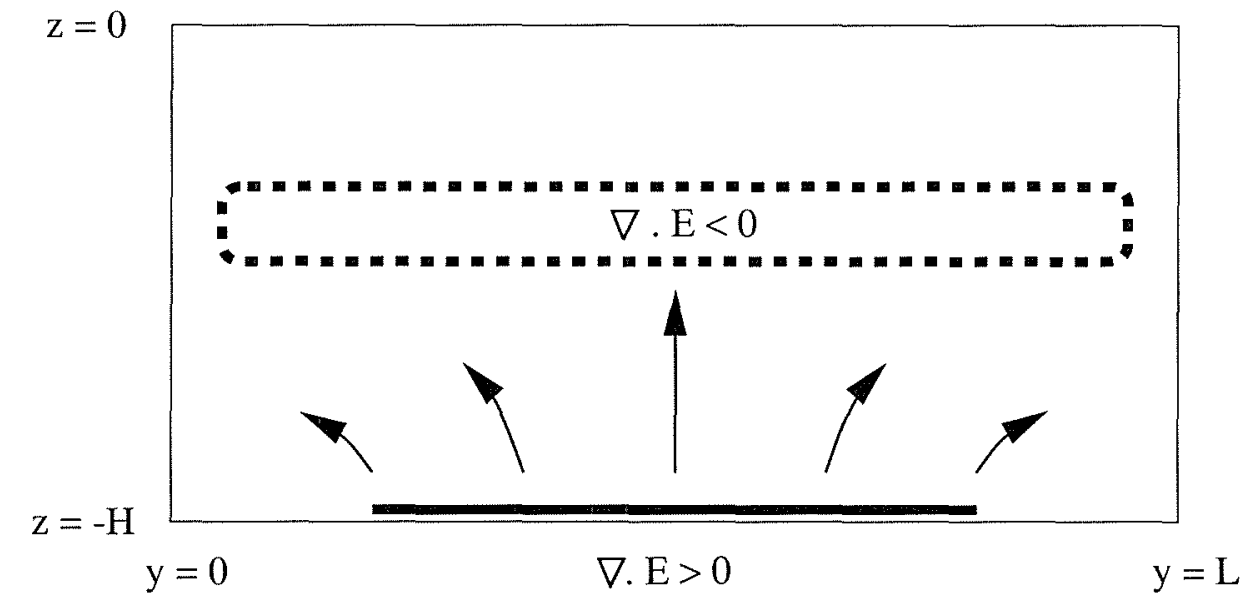

Figure 2.2: Schematic meridional profiles of the Eliassen-Palm flux (arrows) and its divergence (solid and dashed lines) for the most unstable (a) Eady and (b) Charney modes. The thick solid line at the lower boundary represents a Bretherton PV sheet of divergent Eliassen-Palm flux $(\nabla \cdot \mathbf{E}>0)$ arising from the boundary temperature gradients. The compensating convergence $(\nabla \cdot \mathbf{E}<0)$ occurs at the upper boundary in (a) and in the interior in (b) occurring around the steering level. As equation 2.66 shows, the meridional component of $\mathbf{E}$ is equal in size and opposite to the direction of the momentum flux. The vertical component is proportional to the meridional eddy flux of heat. The regions of convergence and divergence have been drawn to have different meridional extent. Thus there has to be a non-zero meridional component of $\mathbf{E}$ and therefore a net lateral transfer of momentum. 
spatial form of the eddy-transfer coefficients.

\subsubsection{The limit of vanishing relative vorticity flux}

It is notable that in the transformed equations, eddy temperature flux divergence terms do not appear on the right-hand-side of equation 2.64.d. This fact lies at the heart of the success of the parameterization of Gent and McWilliams (1990). There, the eddy-flux terms are related to an advective flux rather than to a diffusive process. In so doing, the diffusive nature of height coordinate ocean models, which had compromised them since their inception, was in large part removed. The Gent and McWilliams approach is in fact a limiting case of the methodology applied here, although the implementation is different. In equation 2.64.a the adiabatic nature of the eddy-transfer process is automatically guaranteed because the eddy-terms appear in the momentum, rather than the tracer equations. Thus the advecting velocities are changed by the introduction of an appropriate body force in the momentum equation, rather than explicitly in the tracer equation. What is more, the vorticity and temperature transferring properties of the eddies are handled together and expressed in terms of the eddy transfer of a potential vorticity that is more conserved than either vorticity or temperature alone.

If relative vorticity fluxes are neglected then the eddy meridional flux of potential vorticity given by equation 2.15 reduces to:

$$
\overline{v^{\prime} q^{\prime}}=f \frac{\partial}{\partial z}\left(\overline{\frac{\left(v^{\prime} T^{\prime}\right)}{\bar{T}_{z}}}\right) .
$$

If, as proposed by Gent and McWilliams, the meridional temperature flux is related to the mean meridional temperature gradient thus

$$
\overline{v^{\prime} T^{\prime}}=-K_{T} \frac{\partial \bar{T}}{\partial y},
$$

where $K_{T}$ is the temperature transfer coefficient and is a scalar, then, using thermal wind, equation 2.72 becomes

$$
\overline{v^{\prime} q^{\prime}}=\frac{\partial}{\partial z}\left(\frac{K_{T} f^{2}}{N^{2}} \frac{\partial \bar{u}}{\partial z}\right)
$$


where $N^{2}$ is the buoyancy frequency. The transformed zonal momentum equation can then be written:

$$
\frac{D^{\star} \bar{u}}{D t}-f \bar{v}^{\star}=\bar{F}^{x}+\frac{\partial}{\partial z}\left(\frac{K_{T} f^{2}}{N^{2}} \frac{\partial \bar{u}}{\partial z}\right)
$$

which is the zonal mean equivalent of equation 24 in Gent et al (1995). It shows that in this limit the eddy potential vorticity flux is equivalent to a vertical diffusion of zonal momentum with a coefficient $K_{T} f^{2} / N^{2}$. This has been discussed previously; see, for example, Rhines and Holland (1979), Rhines and Young (1982), Greatbach and Lamb (1990) and Marshall et al (1993).

In Gent and McWilliams (1990) the momentum equations are not transformed. They remain the Eulerian mean equations with the Reynolds stresses represented by Fickian diffusion terms. Temperature and tracer are advected with an 'effective transport' velocity (a term coined from Plumb and Mahlman (1987)) which is explicitly calculated from the large-scale fields. The GM parameterization scheme has been the subject of much recent discussion (see, for example, Tandon and Garret (1996), Treguier et al (1997), Visbeck et al (1996)) and modified approaches have been offered for prescribing the 'bolus' velocity (e.g. McDougall and McIntosh (1996) and Dukowicz and Greatbatch (1997)). It is well documented that GM leads to marked improvements in the ability of height coordinate models to capture and maintain water mass distributions: see, for example, Böning et al (1995), Danabasoglu and Mcwilliams (1995), Robitaille and Weaver (1995), England (1995), and Hirst and McDougall (1996). However, there still remains the need for the representation of vorticity and momentum transport by geostrophic eddies. Gent and McWilliams (1996) address this issue by considering, as here, the transformed Eulerian mean equations. However, in their equations 8-9 the residual mean circulation is not a prognostic variable as in our equation 2.64.b and so has to be explicitly calculated using a closure assumption. Moreover, instead of parameterizing the eddy PV flux, they parameterize the individual components of the Eliassen-Palm momentum flux in terms of downgradient momentum diffusion together with a Coriolis term. Therefore any unmixing of momentum - upgradient transfer - will not be captured. 


\subsection{Summary}

We have performed a mathematical transformation of the Eulerian mean equations. This transformation was combined with the assumption that the eddy field is quasigeostrophic and hydrostatic in nature. The result was a set of governing equations for the transformed Eulerian mean flow in which the eddy term appears a PV flux acting as a body force in the momentum equations. By using the complete TEM framework, we are able to encapsulate both the heat and vorticity transporting properties of the eddy field without having to parameterize them separately, provided that we focus on the eddy transfer of potential vorticity.

The prognostic and advective velocity is that of the residual mean circulation - a movement of fluid parcels that is associated with diabatic processes. Under certain conditions it is equal to the effective transport velocity identified by Plumb and Mahlman (1987) as the relevant velocity for meridional atmospheric tracer transport.

A limiting case of our approach for zonal mean flows, leads to a different implementation of the scheme advocated by Gent and McWilliams (1990). Their parameterization has lead to improvements in water mass distributions and transport because they transform the temperature equation so that the eddy temperature flux terms become implicit. However, because the momentum equations are not also transformed, vorticity is arbitrarily transferred down its mean gradient.

The procedure undertaken was to redefine the mean velocity field, and then systematically scale the remaining eddy terms. The steps are summarized in Figure 2.3. 


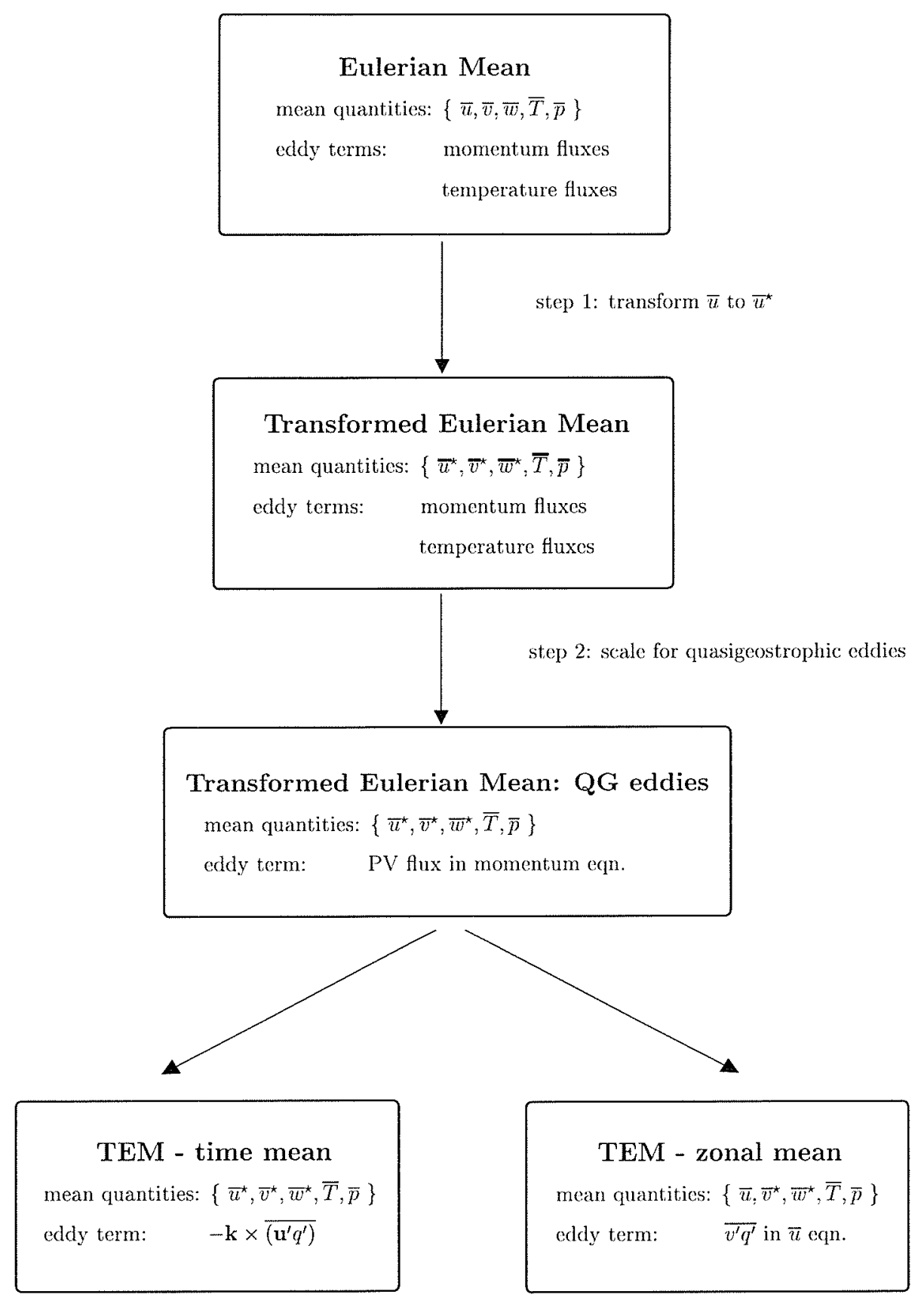

Figure 2.3: Schematic diagram of the procedure followed in chapter 2. The two steps shown yield sets of governing equations for the transformed Eulerian mean flow in which the eddy term appears a PV flux acting as a body force in the momentum equations. The prognostic and advective velocity is that of the residual mean circulation. 


\section{Chapter 3}

\section{Closure for the eddy PV flux}

\subsection{Introduction}

In chapter 2, we found that the application of TEM theory and an assumption about the eddies, led to a set of equations in which the eddy terms appear symbolically as a PV flux. This term acts as a body force term in the momentum equations. This chapter presents the method of closure for the eddy PV flux in terms of the mean flow parameters, if it is not explicitly resolved in the model. I have chosen to present this in a separate chapter to emphasize the fact that the closure assumption is independent of the TEM approach.

\subsection{Downgradient PV transfer}

The subject of parameterization and closure is one that has been studied often [see the textbooks by Tennekes and Lumley (1972) and Stull (1988)] mainly because solutions have to be found on a case-by-case basis. The course pursued here will be a diagnostic approach referred to as "first-order closure". It is a diagnostic approach because no prognostic equation is used to predict for the eddy PV flux. Instead closure for the eddy term will depend on the size and structure of the large-scale PV field diagnosed from the model. "Mixing length theory" is applied to the PV transfer problem at hand, which 
results in a "flux-gradient" relationship for the eddy transfer of PV. The mixing length hypothesis is a kinematic argument, and considers the displacement of a parcel of fluid which then "mixes" its characteristic PV with its surroundings.

If a fluid parcel, and its associated PV, is displaced in the meridional direction between two points $y=0$ and $y=y+\Delta y$, then the ambient PV will have changed by an amount - $\Delta q$. If we assume that the PV perturbation associated with the parcel is $q^{\prime}$ which is $\sim \Delta q$, and also assume that the transport length scale is of the same order as a length scale characterizing the mean flow $l$, then

$$
\frac{q^{\prime}}{l^{\prime}} \sim-\frac{\partial q}{\partial y}
$$

and so

$$
q^{\prime} \sim-l^{\prime} \frac{\partial q}{\partial y} .
$$

Hence, the mean eddy PV flux is given by

$$
\overline{v^{\prime} q^{\prime}}=-K \frac{\partial \bar{q}}{\partial y}
$$

where

$$
K \sim \overline{v^{\prime} l^{\prime}}
$$

is the eddy transfer coefficient. ${ }^{1}$ Equation 3.3 is a kinematic condition. At this point the problem is not closed. To do so demands that the transfer coefficients be specified. This requires knowledge about the dynamics of the eddy transfer such as the mechanism for eddy generation, the eddy velocity scale, and eddy length scales.

From equation 3.4, we can see why mixing length arguments fail, as noted in chapter 1 , for the case of momentum transfer. The dynamics of the momentum transfer have to be considered, because pressure gradients can change $v^{\prime}$ significantly during the eddy displacement. Indeed this is true for any non-conserved or non-quasi-conserved quantity the non-conservative term will, in general, change the quantity of interest during transfer.

\footnotetext{
${ }^{1}$ Note: The $K$ is termed an "eddy transfer coefficient" rather than an "eddy diffusivity". The reason for this will become apparent later.
} 
The result of the non-conservative terms are to invalidate equation 3.1. However, due to the recasting of the equations of motion, it is no longer necessary to deal with the problem of how to represent the eddy momentum flux divergence, because we no longer have to separately parameterize the eddy transfer of momentum and heat.

Extending equation 3.3 to three-dimensions, we have the flux-gradient relation:

$$
\overline{\mathbf{u}^{\prime} q^{\prime}}=-\mathbf{K} \cdot \nabla \bar{q},
$$

where $\nabla=\left(\partial_{x}, \partial_{y}\right)$. This approach for PV transfer has also been employed by many investigators [e.g. Green(1970); Rhines (1977); Marshall (1981); Rhines and Young (1982); Pavan and Held (1996)].

The eddy transfer coefficients of PV are

$$
\mathbf{K}=\left[\begin{array}{ll}
K_{u x} & K_{u y} \\
K_{v x} & K_{v y}
\end{array}\right],
$$

and can be considered to be comprised of two parts:

$$
\mathbf{K}=\mathbf{K}_{i s o}+\mathbf{K}_{a d v}
$$

where $\mathbf{K}_{\text {iso }}$ is symmetric (diagonal elements) and is associated with isobaric mixing, and $\mathrm{K}_{a d v}$ is anti-symmetric (off-diagonal elements) and is associated with eddy advection (Plumb and Mahlman (1987)).

Note that the quasigeostrophic PV transfer is two-dimensional, acting only in the horizontal (along isobaric surfaces). This two-dimensional transfer nature is also true for Ertel PV which acts along isentropic surfaces - see the impermeability theorem in Haynes and McIntyre (1990). This is a tremendous advantage over having to parameterize a field - such as temperature - that is transfered in three-dimensions. For baroclinic eddy heat transport in the atmosphere and ocean, the vertical component of the flux is upgradient. As a consequence, the transfer coefficients manifest themselves as a tensor quantity which results in a vector transport that is directed downgradient. In contrast, due to the horizontal mixing of $\mathrm{PV}$, the $K$ 's in equation 3.6 are scalar quantities which can vary 
spatially and temporally, Green (1970). This two-dimensional nature of PV transfer will prove to be of tremendous benefit, when interpreting the structure and size of the $K$ 's determined from eddy-resolving calculations.

\subsection{Specification of the $K$ 's for zonal mean flows}

As highlighted in chapter 2, the momentum constraint for time-mean flows is not as clear as for the zonal mean flow problems. This is because in the time mean theory, the global volume integral of the eddy PV flux does not necessarily vanish. As a result, in this thesis, a parameterized model will only be developed for zonal mean flows where eddy closure is at its most tractable.

As stressed by equation 2.71, any parametric representation of the eddy flux of potential vorticity must be applied with care to ensure zonal momentum conservation. This is because the zonal mean meridional eddy flux of potential vorticity over the volume of fluid vanishes. That is, the total zonal momentum can be changed only by external forces and friction, and not by the internally generated eddies. This provides an integral constraint on the eddy PV flux term;

$$
\int_{-H}^{0} \int_{0}^{L_{y}} \overline{v^{\prime} q^{\prime}} d y d z=0
$$

Substituting equation 3.5 into equation 3.8 , the condition can be expressed as a constraint on the $K$ 's thus:

$$
\int_{-H}^{0} \int_{0}^{L_{y}} K_{v y} \bar{q}_{y} d y d z=0
$$

where $K=K_{v y}$. The transfer coefficients must be chosen in order to satisfy equation 3.9 .

The determination of the spatial structure of $K$ is admittedly a problematic feature of the flux-gradient relationship. Conventionally the horizontal and vertical structure of the transfer coefficient have assumed to be of separable form. The horizontal structure can be given by the large-scale flow, such as a dependence on a Richardson number or mean 
velocities or velocity shears. A crucial issue is the question of what is the appropriate horizontal length scale that is characteristic of the eddy transfer. In the parameterization problem of mid-latitude synoptic scale eddies in the atmosphere, Green (1970) chose the characteristic length scale to be the width of the baroclinic zone. In contrast, Stone (1972) argued for the mixing length scale to be that of the deformation radius. Simmons (1974) suggested that the appropriate scale should be the geometric mean of the two lengths. However, in the atmosphere all three are on the same order and so the model results are not critically sensitive to the choice made. In comparison however, the problem of eddy transfer length scales in the ocean is much more complicated and is still the subject of much work [Visbeck et al. (1996))].

The choice of the vertical dependence is even more problematic. The simplest possibility is a $K$ that is independent of depth. If this were the case, the momentum constraint (equation 3.9) would only be satisfied for special distributions of $\bar{q}$. Further, it would make more physical sense if the transfer coefficients were allowed to vary in the vertical to reflect differing PV gradients with depth. For example, at a level where the PV gradients are relatively strong compared to other depths, we would expect a small value of $K$. This is because the eddy motion, and therefore property transfer at this depth is more constrained by the presence of the strong gradients.

There is one constraint and so we have one free parameter with which to ensure that equation 3.9 is obeyed for our closure scheme. We choose to specify the $K$ 's as follows:

$$
K(y, z, t)=\kappa_{\text {ref }} Y(y) T(t)\left(1+\gamma \frac{z}{H}\right) .
$$

Here $\kappa_{r e f}$ is a reference value which depends on the nature of the flow [for example as in Visbeck et al.(1996)], $Y(y)$ prescribes the meridional structure, and $T(t)$ the temporal form. The vertical structure is assumed to be linear with a scale height of $H / \gamma$ where $H$ is the total depth of the fluid and $\gamma$ is the free parameter which will be chosen so that equation 3.9 is satisfied. Substituting equation 3.10 into equation 3.9 we obtain an 
expression for $\gamma$ which satisfies the momentum constraint:

$$
\gamma=-\left(\iint Y(y) \bar{q}_{y} d y d z\right) /\left(\frac{1}{H} \iint z Y(y) \bar{q}_{y} d y d z\right) .
$$

$\gamma$ is a measure of the depth-weighted mean PV gradients, and so the vertical profile of the transfer coefficients depend on the PV distribution. Calculation of the PV gradients, $\bar{q}_{y}$, and specification of $\mathrm{Y}(\mathrm{y})$ (either through a analytical function or through dependence on the large-scale flow fields) allows determination of $\gamma$ and therefore the transfer coefficients, $K$. At the side walls eddy transfer is inhibited and as a result the $K$ 's are set to zero, preferably with a taper profile to avoid generating numerical noise.

With knowledge of the transfer coefficients, equation 3.5 closes for the eddy potential vorticity flux, and thus the divergence of the E-P flux. Note that, unlike in Gent and McWilliams (1996), we have not attempted to parameterize the component parts of the E-P flux separately, because we do not know how to do so. Instead we have phrased our closure in terms of the PV flux.

It is emphasized that the assumptions of eddy mixing behind the application of equation 3.5 is that the eddies act to "flux down the mean gradient". This is not the same as assuming they act in a "diffusive" manner. Indeed it is suggested that the flux-gradient description is useful for eddy transport even though the eddies act in diffusive and advective manner, Plumb and Mahlman (1987). Even so, the down gradient flux assumption remains highly controversial. It cannot be formally proved, and circumstances can arise in which it is not true. It may be useful to regard equation 3.5 as a definition, and then the debate revolves around the transfer coefficients $\mathrm{K}$ - are they positive, and how do they depend on large-scale properties of the flow? 


\section{Chapter 4}

\section{Zonal Mean Flows}

\subsection{Introduction}

To illustrate the ideas outlined in chapter 2 and to test the approach to parameterization presented in chapter 3, we present calculations with a three-dimensional HPE numerical model that resolves the baroclinic eddy field. The zonal-mean problem is studied first, because it is the simplest context in which to explore how to proceed. The rationale is that in these zonal flows, eddy closure, although still a very difficult problem, is at its most transparent due to the inherent spatial symmetry.

We compute the eddy statistics of interest, average zonally and consider them in the light of the theoretical ideas presented in chapters 2 and 3 . We then compare the resolved model with a zonally-averaged one which implements TEM with eddy-PV flux forcing. The numerical model used is described in Appendix A.

Sections 4.2 and 4.3 present and discuss the parameterization approach in a $\beta$-plane channel and compares the parameterized model to an eddy-resolving calculations. First, the flow is driven by wind stress at the surface. while the latter section considers the spindown of a baroclinic zone. Finally, section 4.4 looks at the limits of the parameterized model in the context of tropospheric eddies in the atmosphere. 


\subsection{Eastward Flow in a $\beta$-plane channel}

\subsubsection{The eddy-resolving model}

We simulate the wind-driven flow of an ocean in a periodic channel on a $\beta$ plane of width $500 \mathrm{~km}$, length $1500 \mathrm{~km}$, and depth $4500 \mathrm{~m}$ (see figure 4.1). The calculation can be regarded as a primitive-equation counterpart of the kind studied by McWilliams et al. (1978) quasigeostrophically. It can be considered to be an analogue of a segment of the Antarctic Circumpolar Current, although here our jet is in the northern hemisphere!

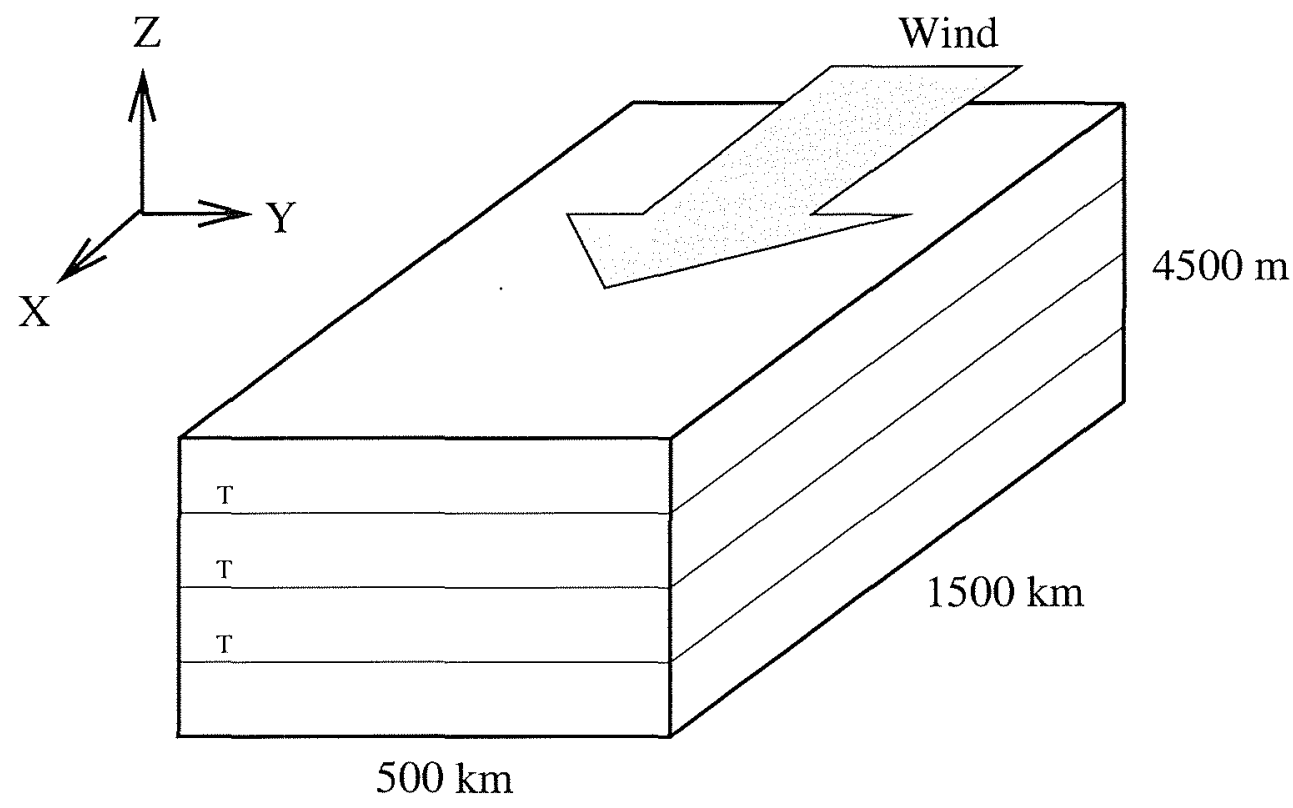

Figure 4.1: A schematic diagram of the channel model domain. A sinusoidal westerly wind stress drives an eastward flow along the axis of the channel

A wind-stress is applied to the upper level of the model of sinusoidal form:

$$
\tau=\tau_{o} \sin \left(\frac{\pi y}{L_{y}}\right) .
$$

It has a maximum value of $0.2 \mathrm{~Pa}$ at the center of the channel and is zero at the side walls. The initial stratification is constant. The vertical grid spacing was $50 \mathrm{~m}$ in the 
upper layer increasing to $400 \mathrm{~m}$ in lower layers. Friction is present through a bottom drag in the lower layer and biharmonic viscosity to suppress numerical noise on the gridscale. There is no thermodynamic forcing $(G=0)$ and no Fickian diffusion terms. Static instability is released by convective adjustment. The numerical experiments carried out in this section are summarized in Table 4.1. The equation of state is a linear function of temperature - henceforth our discussion can be in terms of temperature and temperature flux alone.

Before examining the steady state solution it is appropriate to consider the spin-up of the model from rest. The time development of the surface temperature and velocity fields is shown in figure 4.2. The wind stress drives a southward Ekman flow in the upper layer which returns northward in an Ekman layer at the bottom. This results in downward Ekman pumping in the southern half of the channel and Ekman suction to the north. The resulting meridional overturning leads to a deepening of isotherms in the south and a shoaling to the north. In this way a lateral temperature gradient develops across the channel which supports a surface-intensified jet in thermal wind balance. After a year or so the jet develops growing meanders due to baroclinic instability, as shown in figure 4.2.a. These eddies continue to grow, releasing available potential energy as they reach finite amplitude, figure $4.2 \mathrm{~b}$, until wave breaking occurs and coinciding with a conspicuous decrease in the zonal velocity of the jet. Following the initial instability the eddy field exhibits more irregularity with a broader spectrum of sizes. Finally, after six years or so - see figure 4.2.c - a statistically steady state is reached in which the input of potential energy by the wind is equilibrated by its release through baroclinic instability. The model was integrated for 20 years and the statistically steady state was reached after approximately 6 years. This is indicated by figure 4.3 which plots the times series of the available potential energy per unit mass per unit volume;

$$
P=\frac{1}{2} \frac{\alpha g}{V} \iiint \frac{\left(T-\bar{T}^{x y}\right)^{2}}{\bar{T}_{z}^{x y}} d x d y d z
$$




\begin{tabular}{|c|c|c|c|}
\hline Parameter & units & Eddy-resolving model & Parameterized model \\
\hline$f_{0}$ & $s^{-1}$ & $1 \times 10^{-4}$ & $1 \times 10^{-4}$ \\
\hline Wind-stress, $\tau$ & $\mathrm{Pa}$ & 0.2 & 0.2 \\
\hline Bottom drag & $s^{-1}$ & $1 \times 10^{-5}$ & $1 \times 10^{-5}$ \\
\hline $\mathrm{x}$-domain & $\mathrm{km}$ & 1500 & - \\
\hline$y$-domain & $\mathrm{km}$ & 500 & 500 \\
\hline Depth & $\mathrm{m}$ & 4500 & 4500 \\
\hline Horizontal grid size & $\mathrm{km}$ & 20 & 20 \\
\hline Vertical grid size & $\mathrm{m}$ & $50-400$ & 400 \\
\hline Vertical levels & & 21 & 12 \\
\hline Initial stratification $\left(N / f_{0}\right)$ & & 21 & 21 \\
\hline Rossby radius $\left(\mathrm{NH} / f_{0}\right)$ & $\mathrm{km}$ & 95 & 95 \\
\hline Horizontal diffusivity & $m^{2} s^{-1}$ & 0 & 0 \\
\hline Biharmonic diffusivity & $m^{4} s^{-1}$ & 0 & 0 \\
\hline Vertical diffusivity & $m^{2} s^{-1}$ & 0 & 0 \\
\hline Horizontal viscosity & $m^{2} s^{-1}$ & 0 & 0 \\
\hline Biharmonic viscosity & $m^{4} s^{-1}$ & $1 \times 10^{11}$ & $1 \times 10^{11}$ \\
\hline Vertical viscosity & $m^{2} s^{-1}$ & 0 & 0 \\
\hline$K_{p v}$ & $m^{2} s^{-1}$ & - & 1050 \\
\hline $\mathrm{T}(\mathrm{t})$ & & - & linear ramp: 30 days \\
\hline $\mathrm{Y}(\mathrm{y})$ & & - & $0, y=0, L_{y}$ \\
\hline & & - & $1,0<y<L_{y}$ \\
\hline
\end{tabular}

Table 4.1: Parameters for the eddy-resolving and parameterized stress-driven channel experiments. 

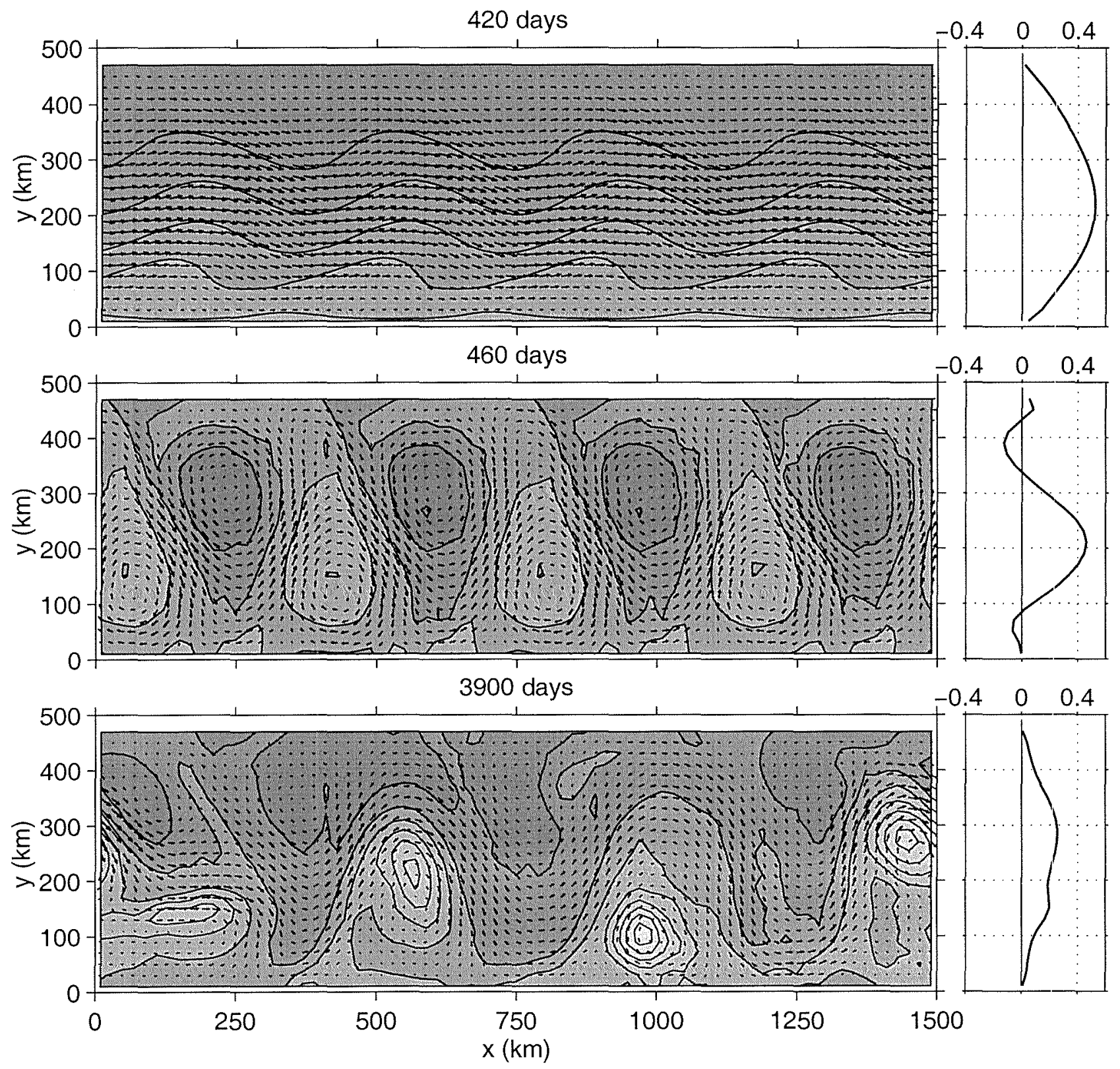

Figure 4.2: Surface velocities from the eddy-resolving model after 420, 460, and 3900 days. The temperature is contoured and shaded with lighter shading denoting warmer lighter water. The panels on the right display the corresponding mean zonal surface velocity in $m s^{-1}$. 
and the kinetic energy per unit mass per unit volume;

$$
K=\frac{1}{2 V} \iiint\left(u^{2}+v^{2}\right) d x d y d z
$$

The time-average was obtained by averaging the last 10 years of integration.

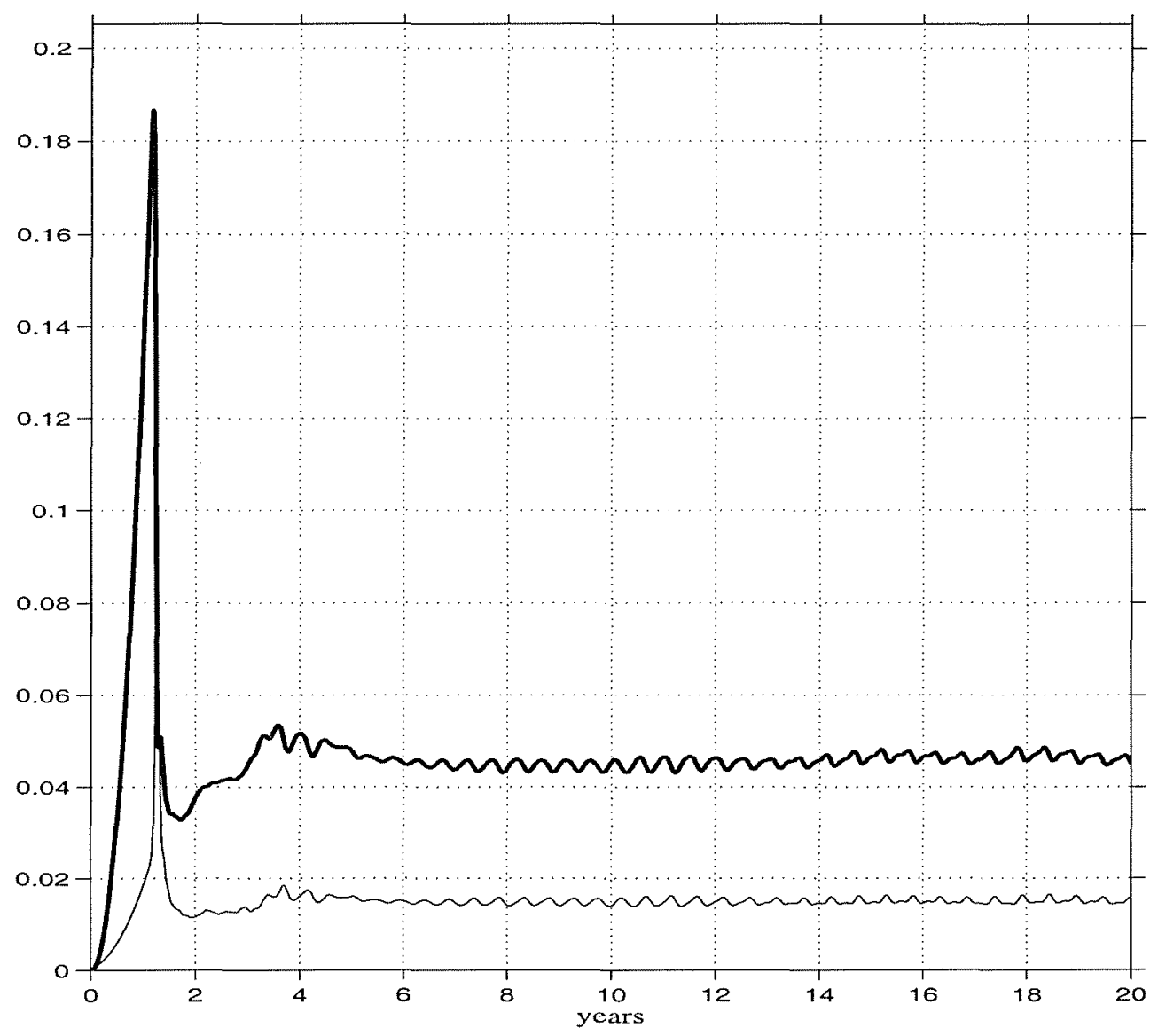

Figure 4.3: The times series of the available potential energy (heavy line) and kinetic energy (thin line) per unit mass per unit volume. The available potential and kinetic energies increase until about day 400, when the ratio APE:KE is 7.5:1 at which point Rossby wave breaking occurs, releasing available potential energy and causing a conspicuous decrease in the available potential and kinetic energies. After six years or so, a statistically steady state is reached where available potential energy is three times the kinetic energy. 


\section{Equilibrated state}

The zonal mean zonal velocity in the equilibrated state is characterized by a surfaceintensified jet (figure 4.4.a) in thermal wind balance with the temperature field in figure 4.4.b. Maximum surface velocities are $0.24 \mathrm{~ms}^{-1}$ in mid-channel, reducing to zero at the side walls. The Eulerian mean meridional streamfunction is plotted in figure 4.5.a and is that of the stress-driven Ekman flow. It consists of southward transport at the surface with sinking in the south and northward return flow at depth. This Eulerian mean flow deepens the isotherms in the south and shallows them to the north, acting to increase the meridional temperature gradient. This stress-driven overturning rate has a maximum in mid-channel of 4.00 Sv. However, in the transformed Eulerian mean framework, equation 2.64.d states that

$$
\bar{v}^{\star} \bar{T}_{y}+\bar{w}^{\star} \bar{T}_{z}=0
$$

in the equilibrated state. If we write the residual mean circulation in terms of a streamfunction;

$$
\chi^{\star}=\chi_{E u l}+\chi_{f l u x}
$$

where the streamfunctions $\chi_{E u l}$ and $\chi_{f l u x}$ are those of the Eulerian mean and buoyancy flux terms respectively. equation 4.4 can now be restated as

$$
J\left(\chi^{\star}, \bar{T}\right)=0
$$

For the stress-driven channel at hand, the only physical solution to equation 4.6 is that there is zero residual mean circulation: $\chi^{\star}=0$. That is the residual mean overturning circulation has vanished. The wind-driven Eulerian mean circulation is exactly canceled by the terms involving the buoyancy fluxes in equation 2.63.a-b. The streamfunction involving the buoyancy flux terms, $\chi_{\text {flux }}$, is plotted in figure 4.5.b and it is opposite in direction and equal in magnitude almost everywhere to the Eulerian mean streamfunction. At any latitude $\chi_{\text {flux }}$ is constant with height except for the upper $500 \mathrm{~m}$ in the

channel center. This overturning rate has a maximum in mid-channel of $3.98 \mathrm{~Sv}$. For the 
(a)

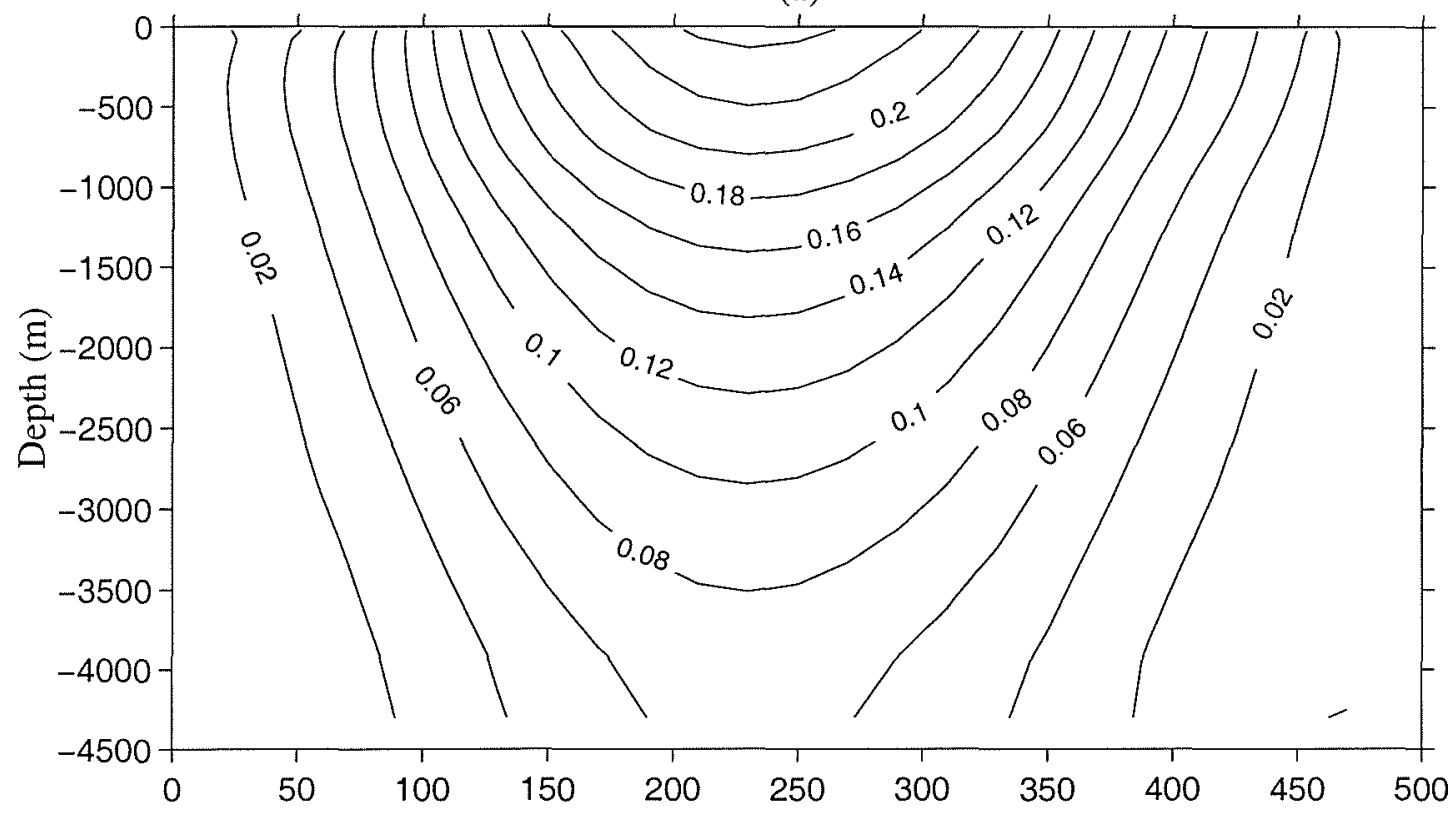

(b)

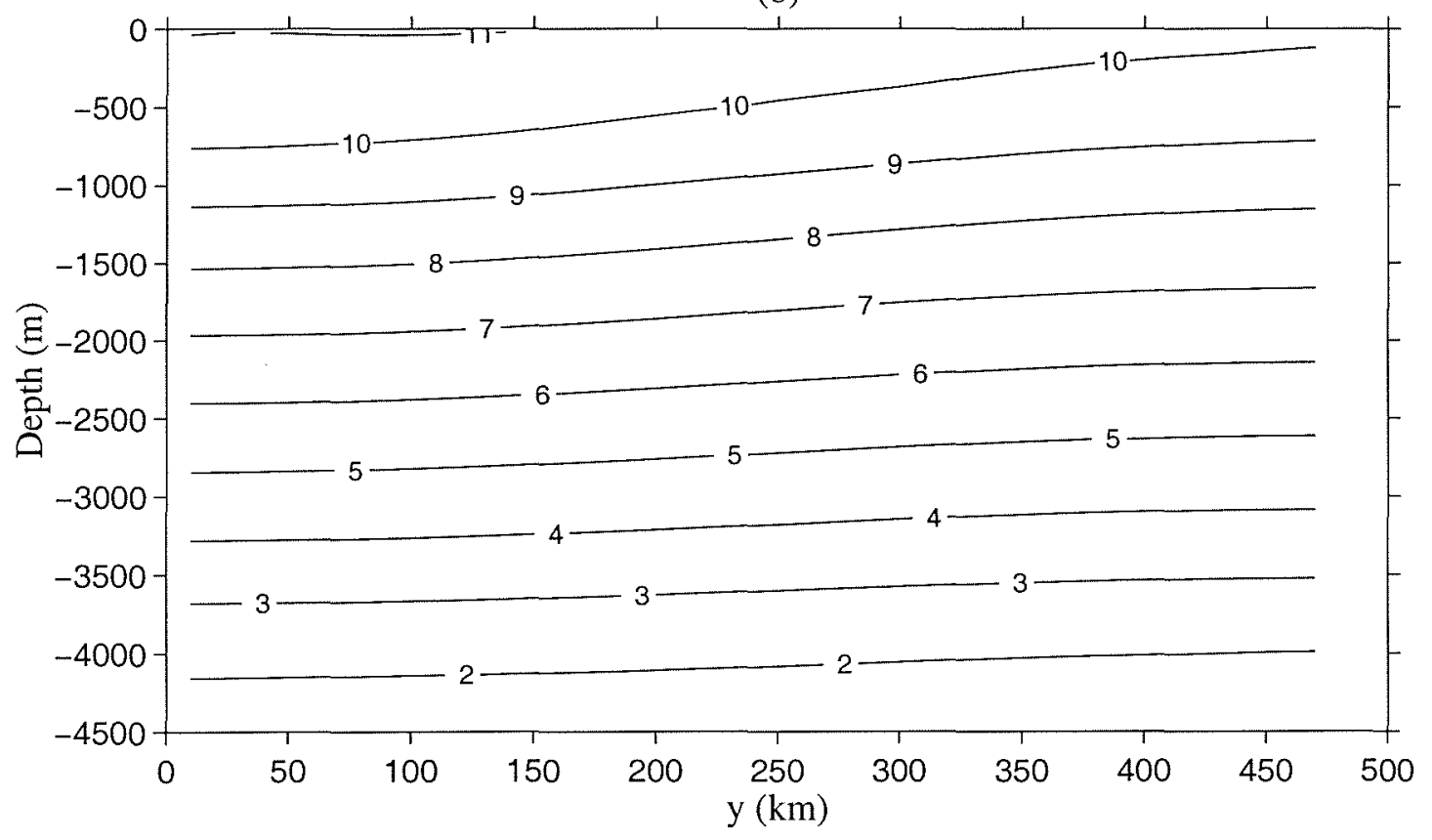

Figure 4.4: The eddy-resolving eastward flow in a $\beta$-plane channel. The time-averaged meridional cross-sections of: (a) zonal mean zonal velocity $\left(\mathrm{ms}^{-1}\right)$; (b) zonal mean temperature. The time average was taken from 10 to 20 years. 
(a)

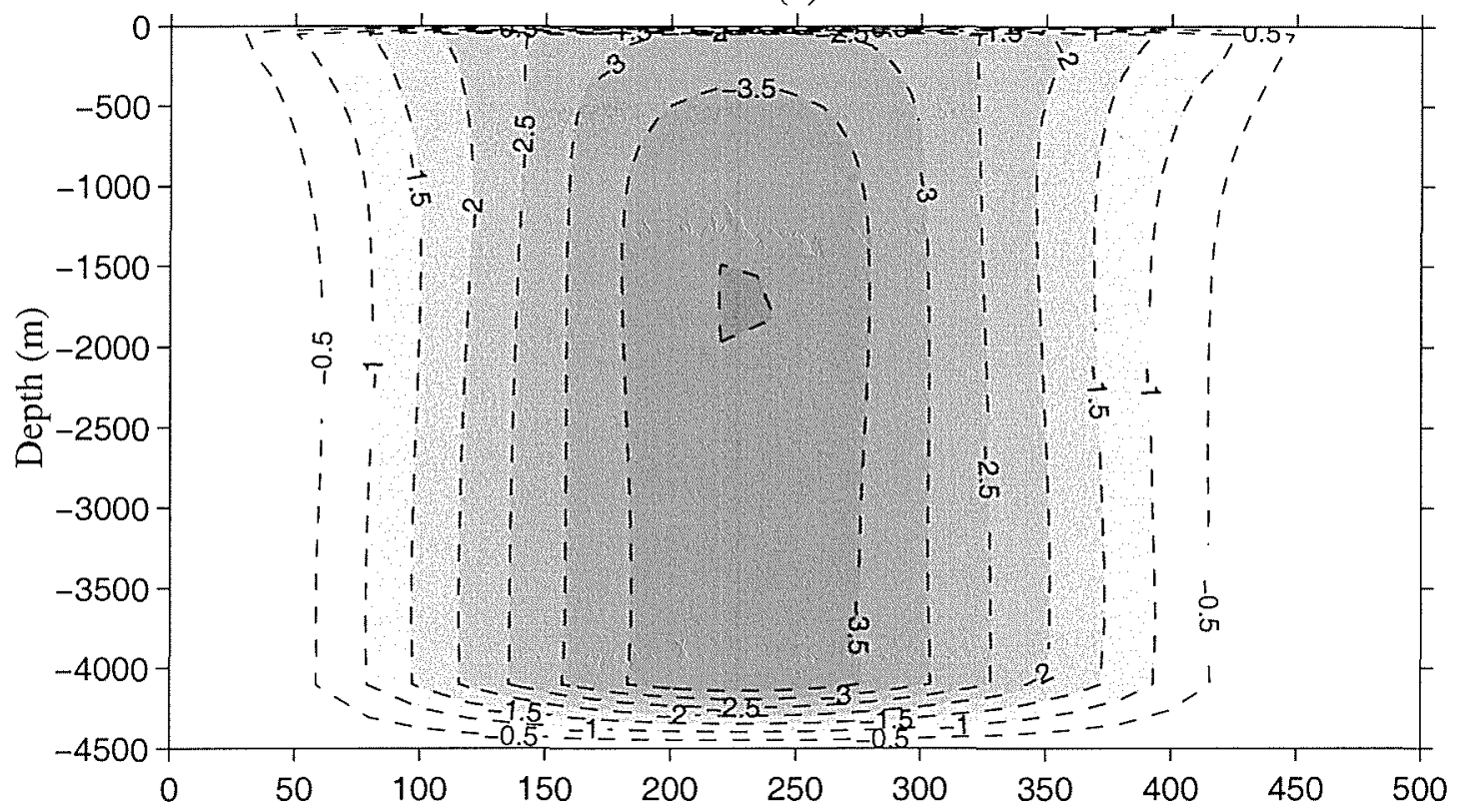

(b)

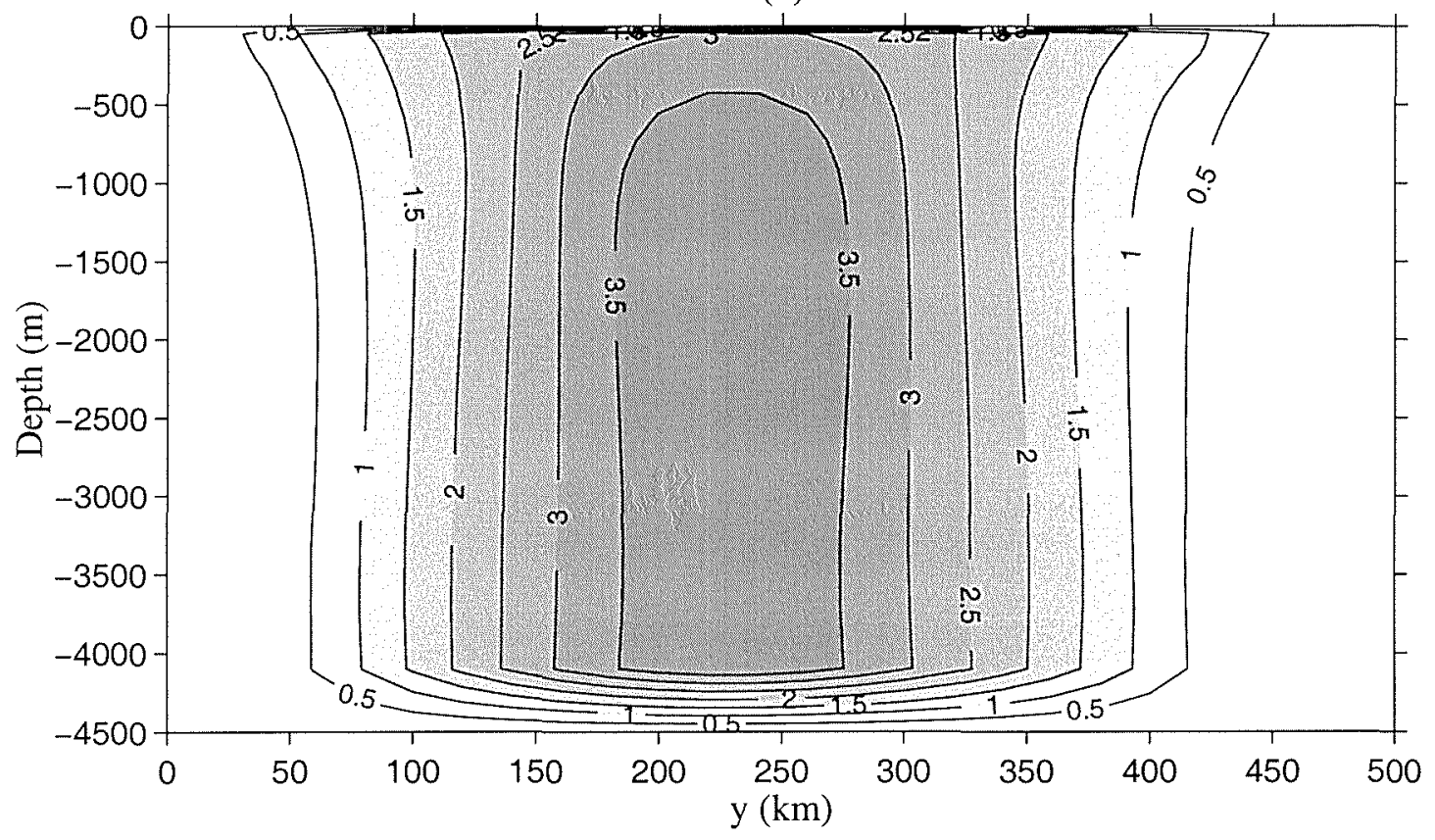

Figure 4.5: The wind-driven Eulerian mean streamfunction, $\chi_{E u l}$, in (a) is almost exactly canceled by $\chi_{\text {flux }}$ in (b). Units are Sv. The result is the near vanishing of the residual mean overturning circulation. 
turbulent, non-linear, primitive equation eddy resolving flow at hand there exists a very small non-zero residual mean circulation (over the time interval of the time averaging), the running average of which asymptotes to zero. Thus the TEM framework provides a clear understanding of the equilibrated zonal mean fields of the stress driven channel.

\section{Eddy statistics and transfer characteristics}

\section{Vorticity and potential vorticity fluxes}

In the steady state the depth-integral of the zonal momentum equation, (equation 2.64.a), is:

$$
\int_{-H}^{0} \overline{v^{\prime} q^{\prime}} d z+\int_{-H}^{0} \overline{F_{x}} d z=0
$$

Using equation 2.65, our definition of $\underline{E}$, equation 2.66 , and noting that $E_{z}$ is zero at the top and bottom boundaries, equation 4.7 yields:

$$
\int_{-H}^{0}-\frac{\partial}{\partial y} \overline{u^{\prime} v^{\prime}} d z+\int_{-H}^{0} \overline{F_{x}} d z=0
$$

Integrating over the channel we have:

$$
\int_{V} F d V=0
$$

Note that at any latitude $\int_{-H}^{0} \overline{F_{x}} d z \neq 0$; the bottom stress does not exactly balance the surface stress. Their difference is equal to the vertically integrated potential vorticity flux, which itself is exactly equal to the vertically integrated relative vorticity flux - see figure 4.6. Thus the effect of the eddies is to transfer eastward momentum into the center of the eastward jet resulting in a zonal mean eastward body force that sharpens and intensifies of the mean zonal flow. Thus eddies pump eastward momentum in to the jet, taking it from the flanks. The net effect of the eddy vorticity transfer, then, is to sharpen the jet with momentum being transferred up its large-scale gradient. This sharpening and intensification is shown by the depth-integrated $\bar{u}$ in figure 4.7. The structure of the eddy momentum flux can be understood in terms of a asymmetry in the horizontal structure 


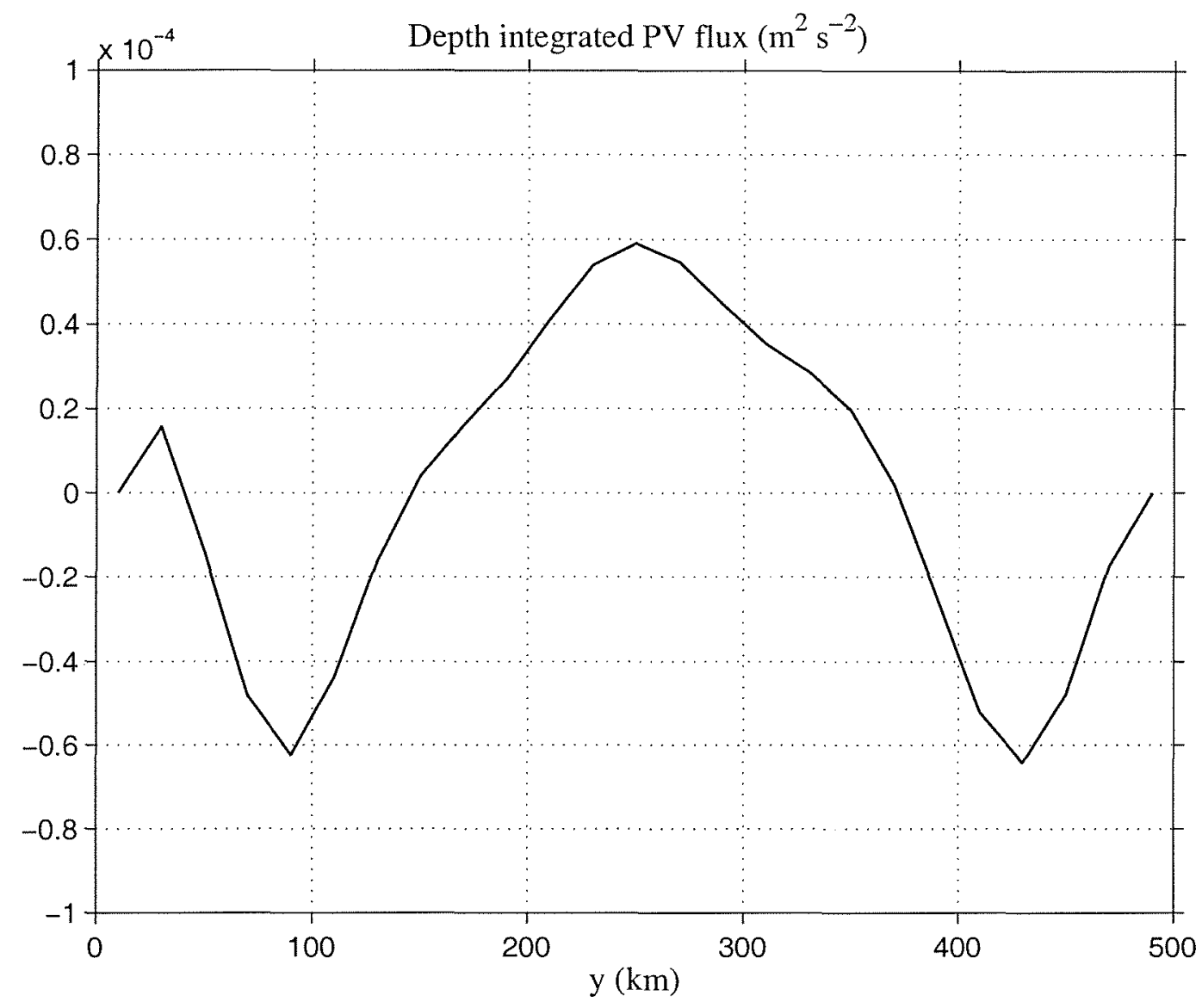

Figure 4.6: The depth integrated PV flux. At any latitude the bottom drag does not exactly balance the surface stress and the difference is balanced by an eddy flux of PV. A positive (eastward) body force is exerted on the zonal flow in the center of the channel and a negative (westward) body force is exerted on the flanks of the jet.

of the eddies. The eddy velocities $\left(u^{\prime}, v^{\prime}\right)$ have a zero zonal mean, but their product can be nonzero if the eddy pressure troughs and ridges display horizontal tilt. Consider a symmetric low pressure perturbation straddling the jet center. The perturbation will begin to propagate westward, but due to the mean zonal velocity in which the eddy disturbance is embedded the westward propagation will depend on meridional position. This will deform the perturbation into a 'banana-shaped' pattern as shown in figure 4.8. To the south of the jet axis the isobars slope in a southwest-northeast sense to give a northward eddy flux of eastward eddy zonal velocity in the zonal mean. North of the jet 


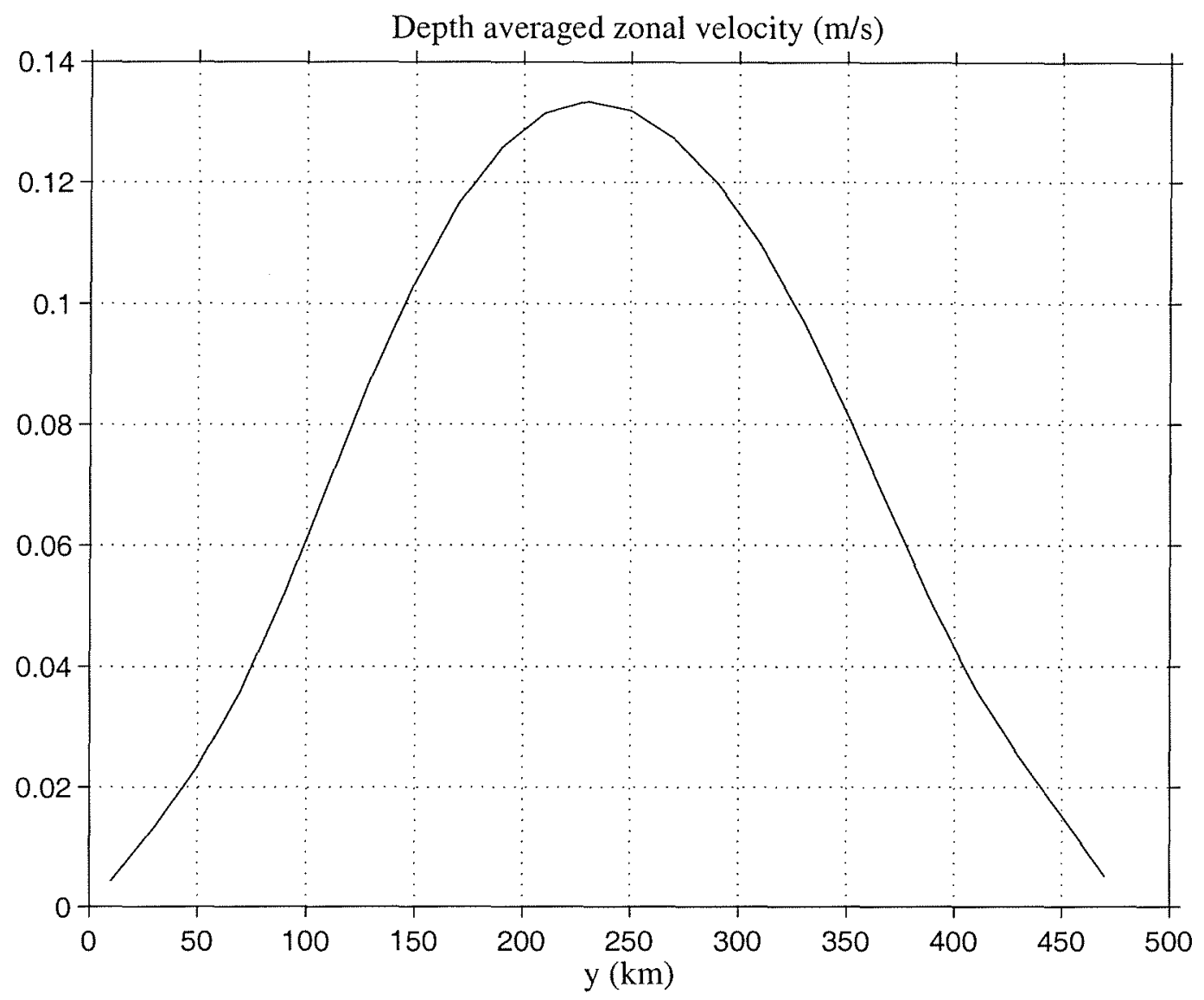

Figure 4.7: The depth average of the zonal mean zonal velocity in the eddy-resolving equilibrated state.

axis, the isobars exhibit a southeast-northwest tilt which in the zonal mean results in a southward eddy flux of eastward eddy zonal velocity.

We now examine the eddy-flux quantities of the eddy resolving flow. The budget for the eddy potential vorticity variance (enstrophy) with the quasigeostrophic scaling for the eddies is

$$
\left(\frac{q^{\prime 2}}{2}\right)_{t}+\mathbf{v}^{\prime} q^{\prime} \cdot \nabla \bar{q}+\overline{\mathbf{v}} \cdot \nabla\left(\frac{q^{\prime 2}}{2}\right)=q^{\prime} D
$$

where $D$ are dissipative terms. Figure 4.9. a shows the surface velocities and temperature contours after 3660 days. The corresponding surface maps of $\mathbf{v}^{\prime} q^{\prime} \cdot \nabla \bar{q}^{x}$ (figure 4.9.b) shows that instantaneously $\mathbf{v}^{\prime} q^{\prime} \cdot \nabla \bar{q}^{x}$ can vary spatially taking both positive and negative values 


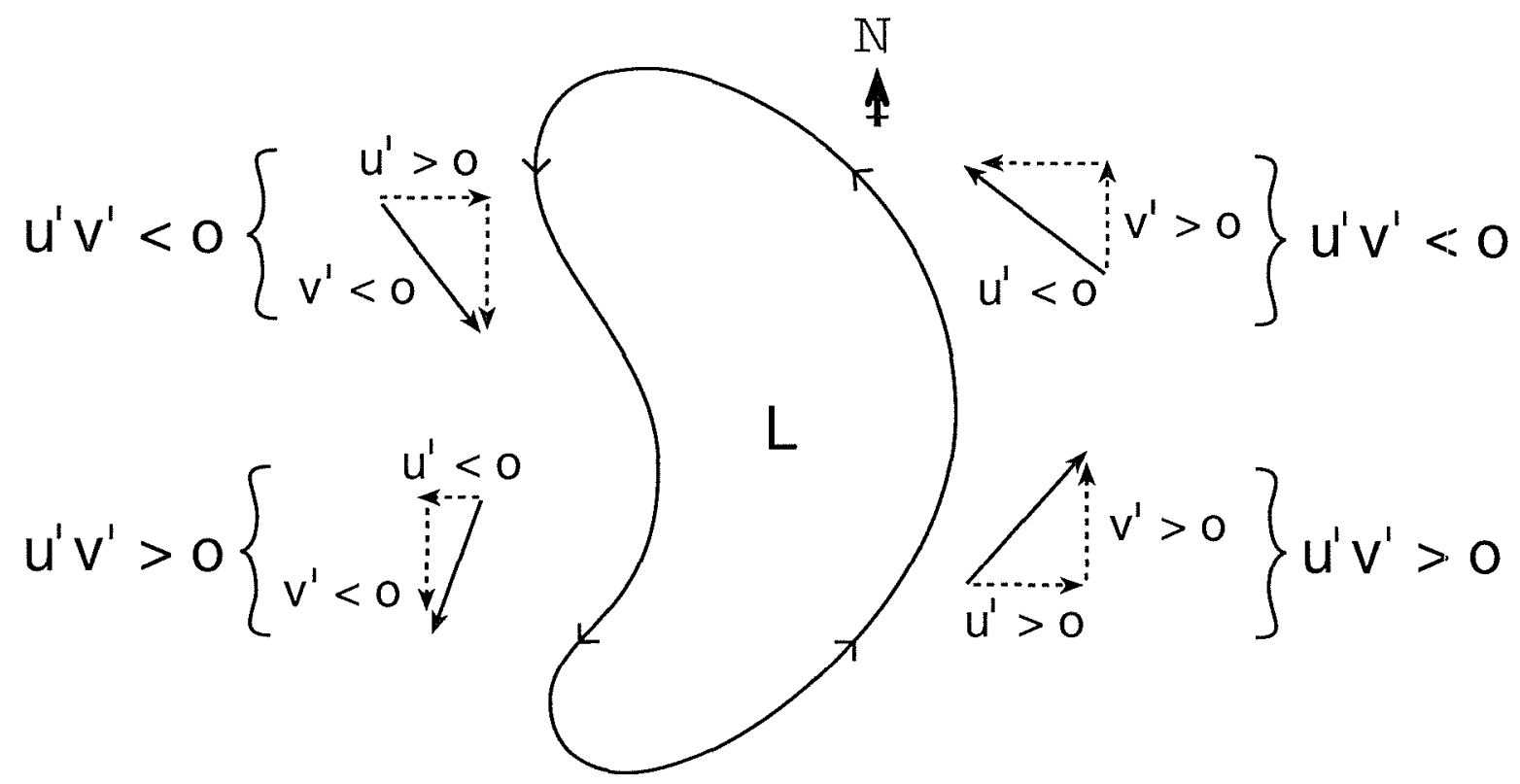

Figure 4.8: Eddy momentum fluxes associated with a 'Banana-shaped' eddy. The eddy velocities $\left(u^{\prime}, v^{\prime}\right)$ have a zero zonal mean, but their product can be nonzero if the eddy, as here, is anisotropic. To the south of the jet axis the trough slopes in a southwestnortheast sense inducing a northward eddy flux of eastward eddy zonal velocity $\overline{u^{\prime} v^{\prime}}>0$. North of the jet axis, the troughs tilt southeast-northwest and $\overline{u^{\prime} v^{\prime}}<0$. Thus the effect of the eddies is to transfer eastward momentum into the center of the eastward jet from the flanks. This effect is well known in the atmospheric literature, see for example, Starr (1968) and Houghton (1977).

depending on whether the eddy disturbance is growing or decaying and/or on advection of PV variance by the mean flow. The zonal mean (figure 4.9.c) at this time has latitudes of $\overline{\mathbf{v}^{\prime} q^{\prime}} \cdot \nabla \bar{q}^{x}$ being positive as well as negative, indicating that instantaneously, potential vorticity transfer may not locally be downgradient (although globally it will be). However, the time average over the last ten years (figure 4.9.d) shows that the PV transfer is down the mean gradient at all latitudes north of $80 \mathrm{~km}$. To the south of $80 \mathrm{~km}$ the mean PV gradient changes sign but ${\overline{\mathrm{v}^{\prime} q^{\prime}}}^{x t}$ does not, due to the finite size of the eddies, and as a result ${\overline{\mathbf{v}^{\prime} q^{\prime}}}^{x t} \cdot \nabla \bar{q}^{x t}$ is positive. Figure 4.10.a plots the meridional profile of ${\overline{v^{\prime} q^{\prime}}}^{x t}$ 
(a)

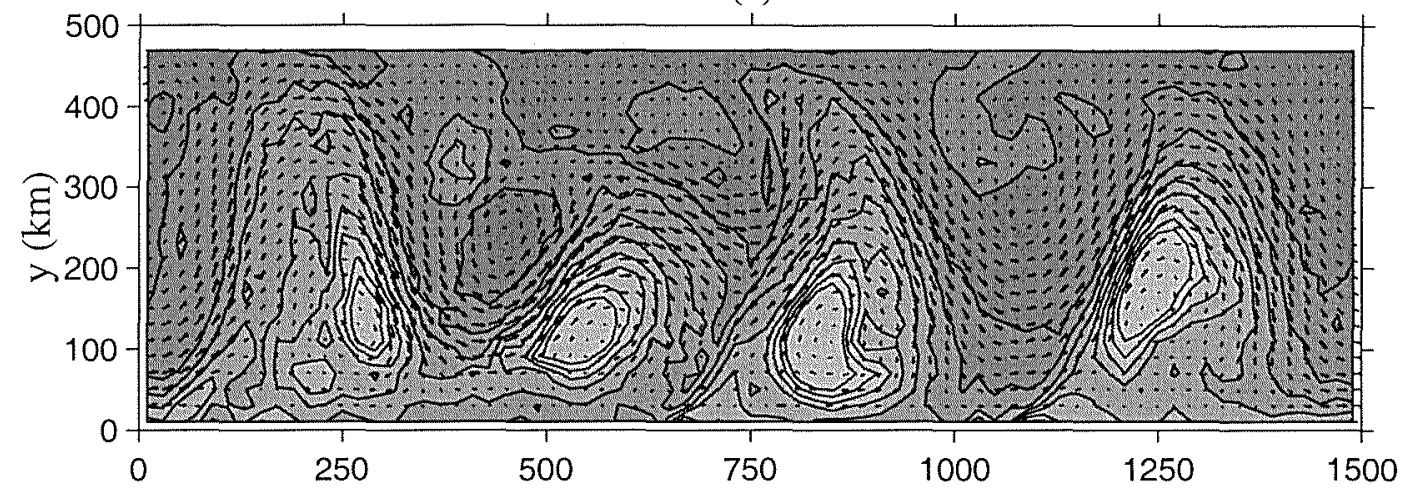

(b)
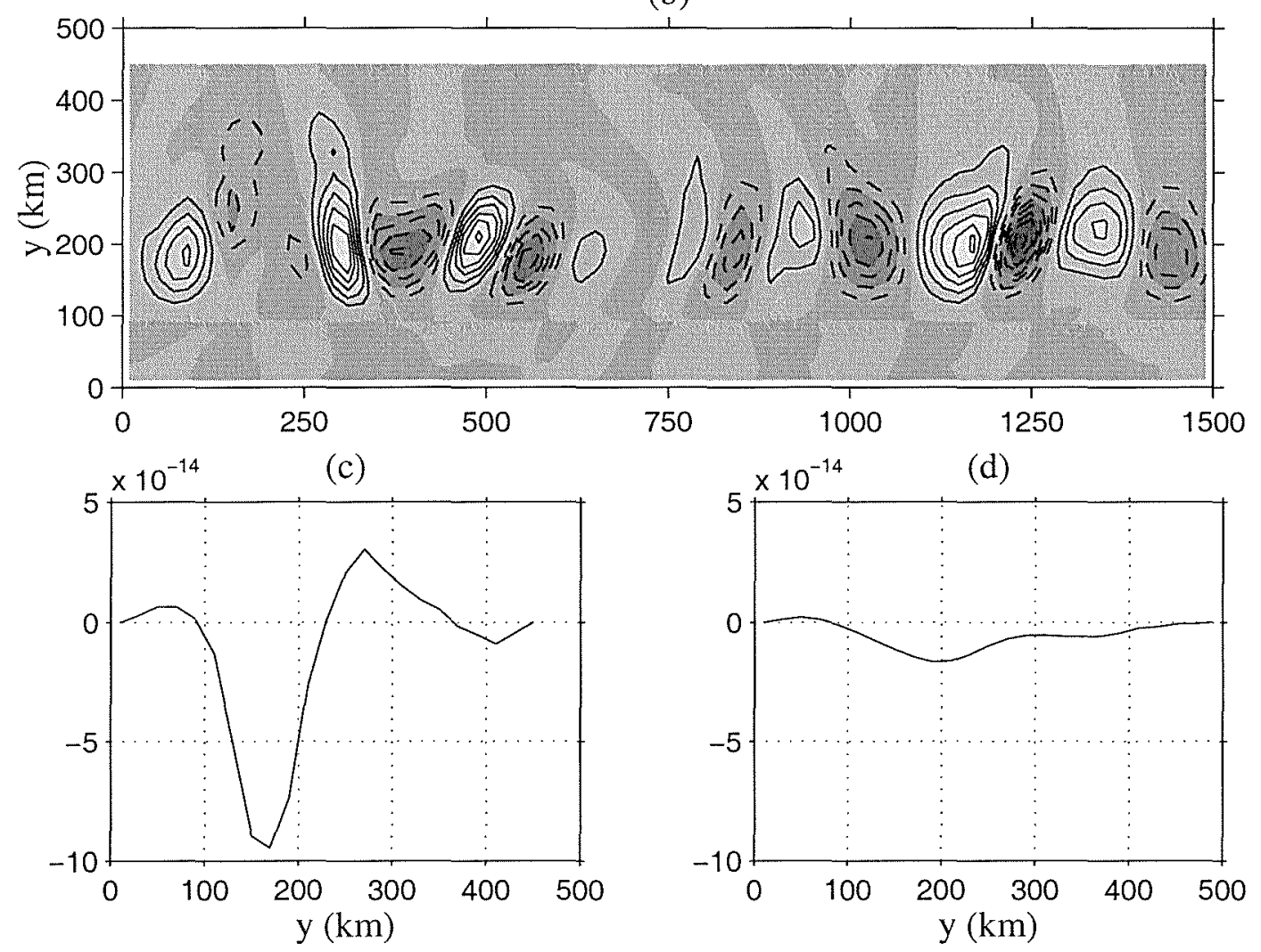

Figure 4.9: (a). Surface velocities from the eddy-resolving model after 3660 days. The temperature is contoured and shaded with lighter shading denoting warmer lighter water; (b) Surface map of $\mathbf{v}^{\prime} q^{\prime} \cdot \nabla \bar{q}^{x}$ after 3660 days. Positive (negative) values are contoured with a solid (broken) line. The contour interval is $5 \times 10^{-13} \mathrm{~s}^{-3}$. (c) ${\overline{\mathrm{v}^{\prime} q^{\prime}}}^{x} \cdot \nabla \bar{q}^{x}$ at this time. (d) time average $\overline{\mathbf{v}^{\prime} q^{\prime}} \cdot \nabla t \cdot \nabla \bar{q}^{x t}$. The negative values indicate downgradient transfer of quasigeostrophic PV. 
diagnosed from the model and shows eddies exert a positive (eastward) body force in the lower sheet and a negative body force in the upper PV sheet. This can be understood when we consider the zonal momentum balance written thus:

$$
\begin{aligned}
& 0={\overline{v^{\prime} q^{\prime}}}^{x t}+\frac{1}{\rho_{o}} \frac{\partial \bar{\tau}^{x t}}{\partial z} \\
& 0={\overline{v^{\prime} q^{\prime}}}^{x t} \\
& 0={\overline{v^{\prime} q^{\prime}}}^{x t}-\epsilon \bar{u}^{x t}
\end{aligned}
$$

for the upper boundary, interior, and the lower boundary of the channel respectively. Thus in the upper layer the imposed wind stress is balanced by a southward eddy flux of potential vorticity. In the lower layer the bottom stress is balanced by a northward eddy flux of potential vorticity. In the interior the meridional eddy flux of potential vorticity is very small and the Eliassen-Palm flux is non-divergent.

The zonal-mean eddy flux of temperature - see figure 4.10.b - is almost constant with height in mid-channel but weakens as the surface is approached. However $\overline{v^{\prime} T^{\prime}} / N^{2}$ is almost constant with depth since $N^{2}$ is weaker in the upper $\mathrm{km}$ - this was exploited by Johnson and Bryden(1989) and Marshall et al.(1993) in their simplified models of the Antarctic Circumpolar Current. The temperature flux characteristics are broadly in accord with the Eady model of baroclinic instability.

\section{Eddy transfer coefficients}

We now inspect the sense of the meridional eddy flux of PV with respect to the mean PV gradients to assess whether the flux-gradient eddy closure hypothesis (equation 3.5) is appropriate. The meridional profile of the transfer coefficients for the upper and lower PV sheets are shown in figure 4.11. All values of $K$ are positive except near the boundary where, as noted before, the PV gradient is negative. In the upper sheet $K$ ranges from $\sim 200 \mathrm{~m}^{2} \mathrm{~s}^{-1}$ in the center of the jet, where the mean PV gradients are a maximum, to $\sim 900 \mathrm{~m}^{2} \mathrm{~s}^{-1}$ at the northern flank where the mean gradients are weaker. On the southern-flank of the jet the diagnosed $K$ is negative where the sign of the mean potential 
(a)

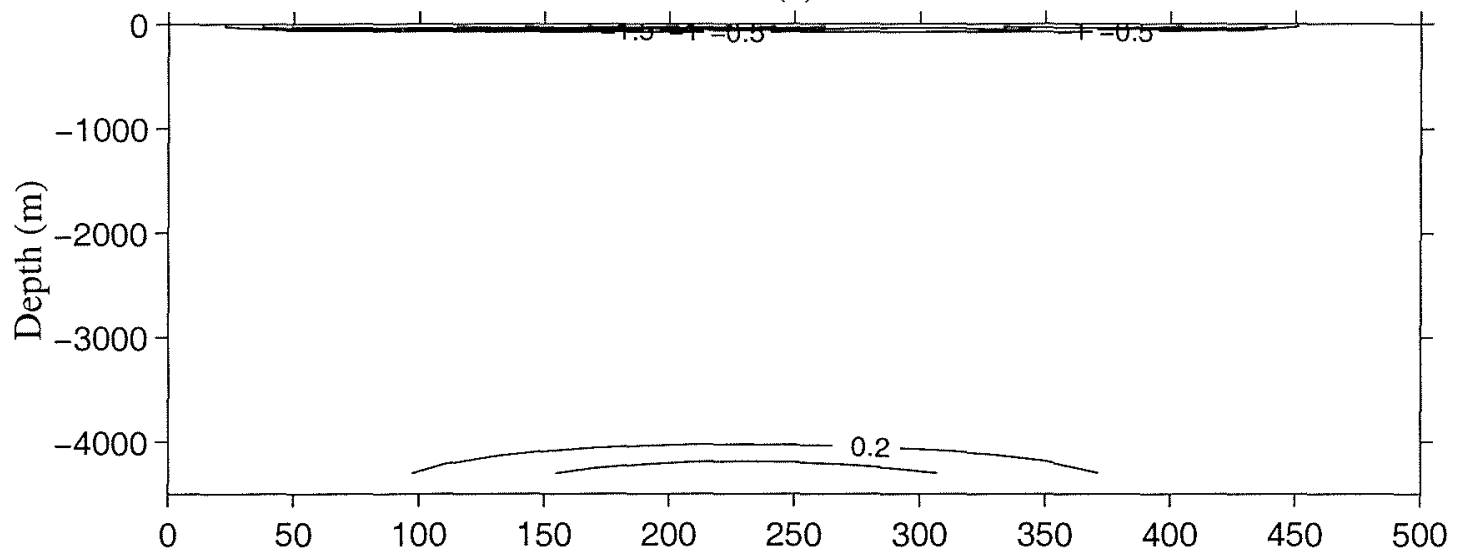

(b)

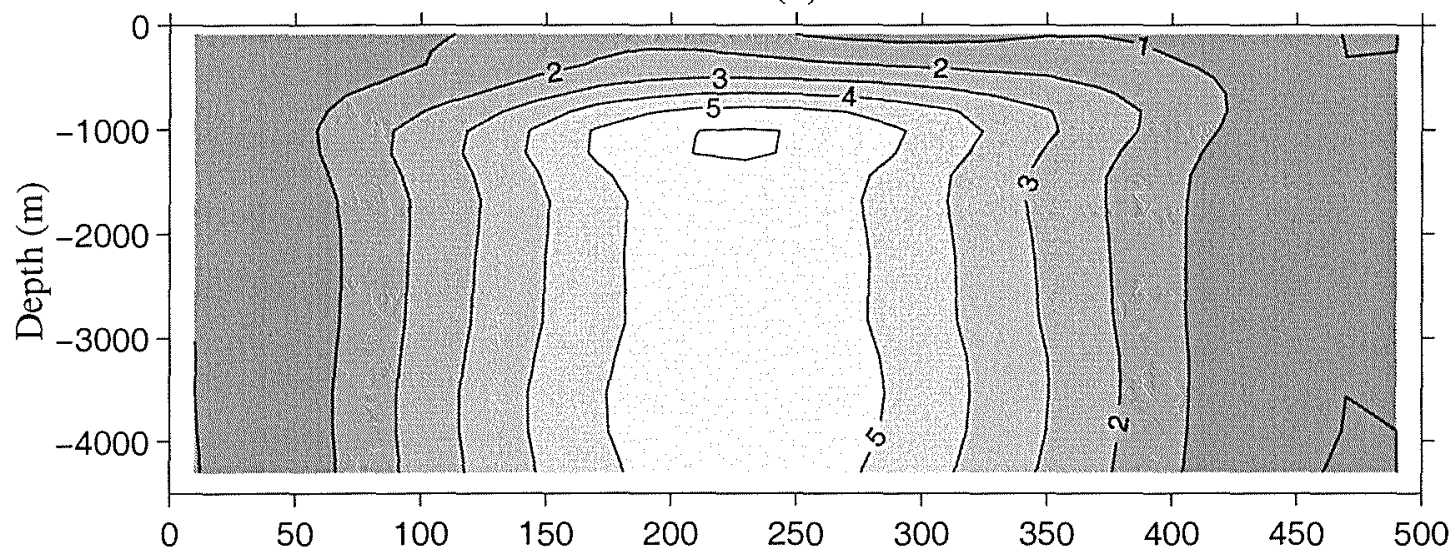

(c)

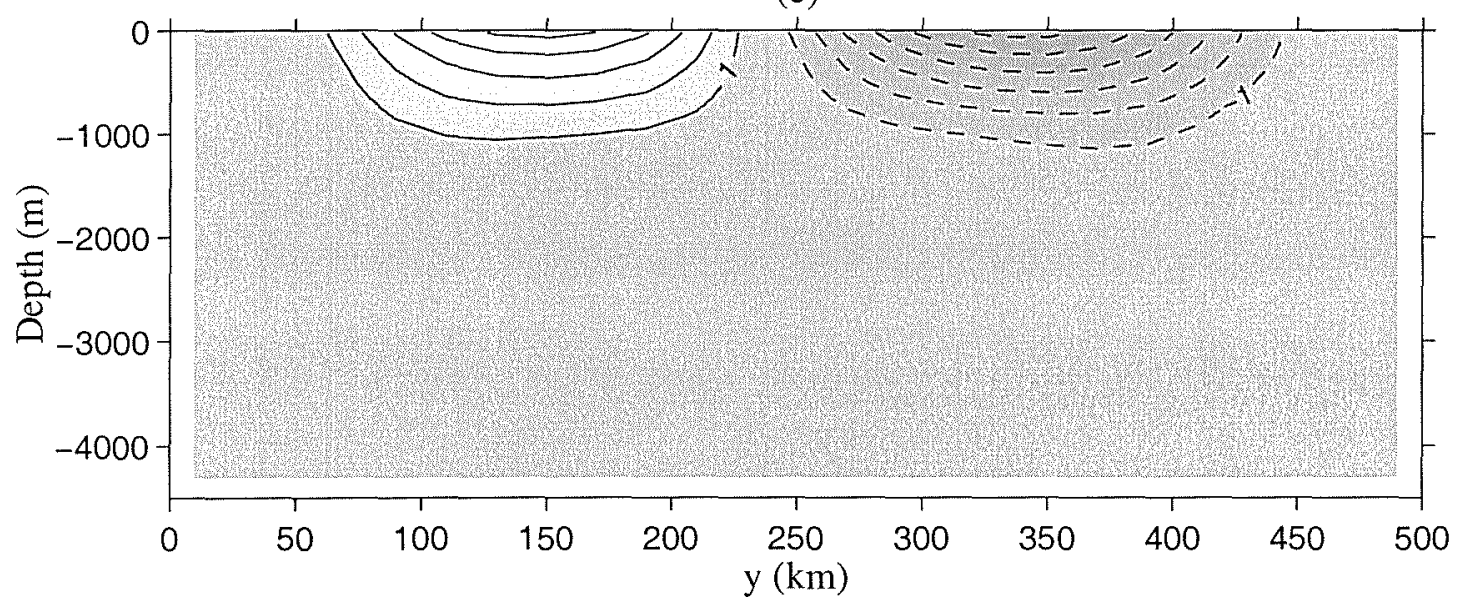

Figure 4.10: Meridional cross-sections of; (a) The eddy PV flux ${\overline{v^{\prime} q^{\prime}}}^{x t}$ is dominated by the boundary sheets with divergence at depth and surface convergence; (b) The eddy-flux of temperature, ${\overline{v^{\prime} T^{\prime}}}^{x t}$. The contour interval is $1 \times 10^{-3} \mathrm{~ms}^{-1} \mathrm{~K}$. (c) The eddy-flux of momentum, $\overline{u^{\prime} v^{\prime}} x t$. The contour interval is $1 \times 10^{-3} \mathrm{~m}^{2} \mathrm{~s}^{-2}$. 


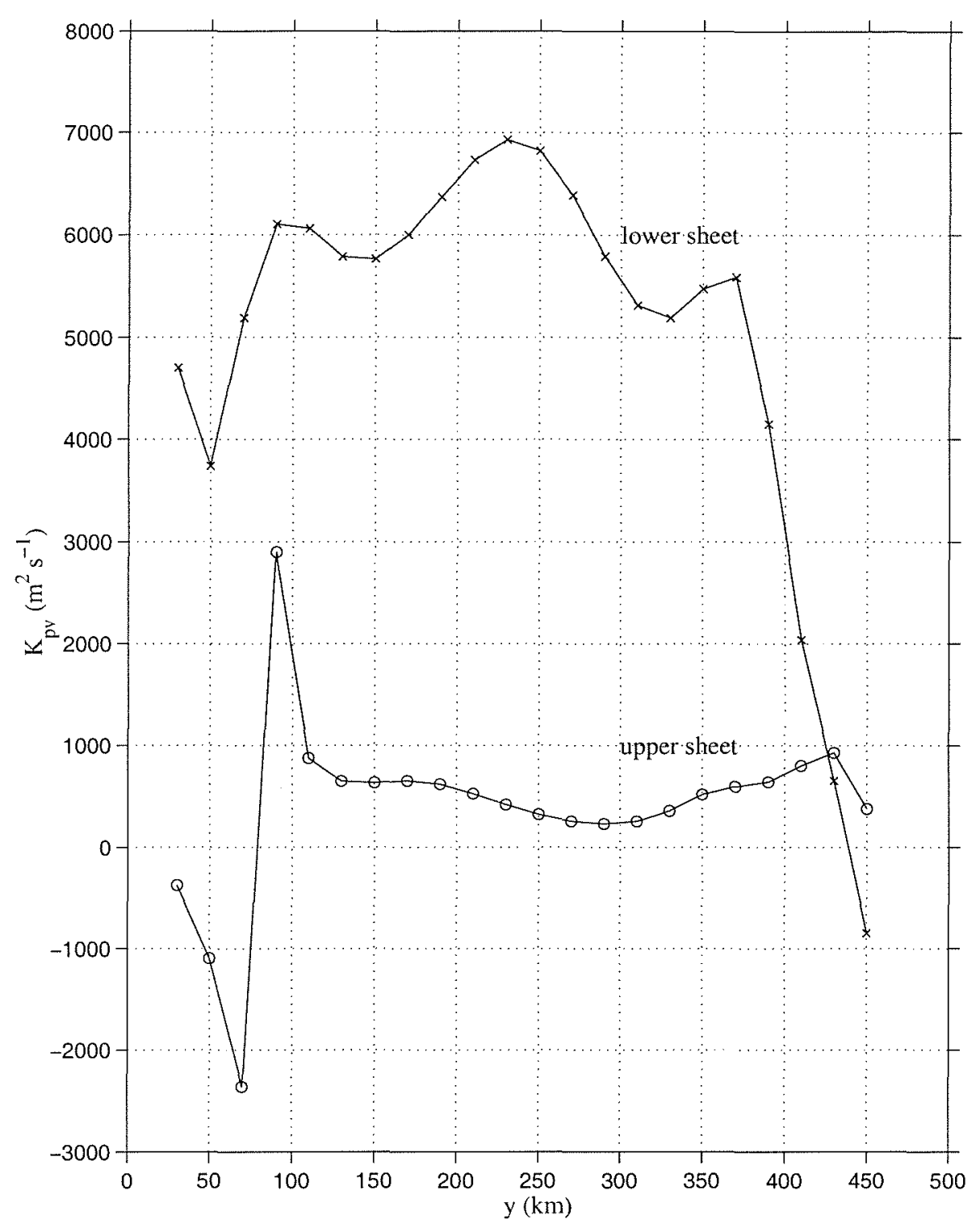

Figure 4.11: The diagnosed transfer coefficients for quasigeostrophic potential vorticity in the upper PV sheet (circles) and the lower PV sheet (crosses) in the statistically steady state.

vorticity gradient changes sign but ${\overline{v^{\prime} q^{\prime}}}^{x t}$ does not. In the lower sheet the values of $K$ are higher than in the upper sheet reaching a maximum value of $\sim 6900 \mathrm{~m}^{2} \mathrm{~s}^{-1}$ in the jet center. A local minimum is found on either side of the jet in regions where the mean PV gradients have slight maxima. Thus the structure of the diagnosed transfer coefficients is quite complex in both the horizontal and vertical. This complexity is further revealed 
when we plot ${\overline{v^{\prime} q^{\prime}}}^{x t}$ against $\bar{q}_{y}^{x t}$ for each sheet (figure 4.12). If the transfer were truly local and directed downgradient then the slope of $\overline{v^{\prime} q^{\prime}}$ vs. $\bar{q}_{y}^{x t}$ would be $-K$. Figure 4.12 shows that for the upper sheet the line is not straight but rather doubles back to form a partly open curve suggesting that for any particular value of the gradient there are two values of eddy PV flux. This is because different values of $\bar{q}_{y}$ occur on either side of the jet center, and have different eddy fluxes associated with them.

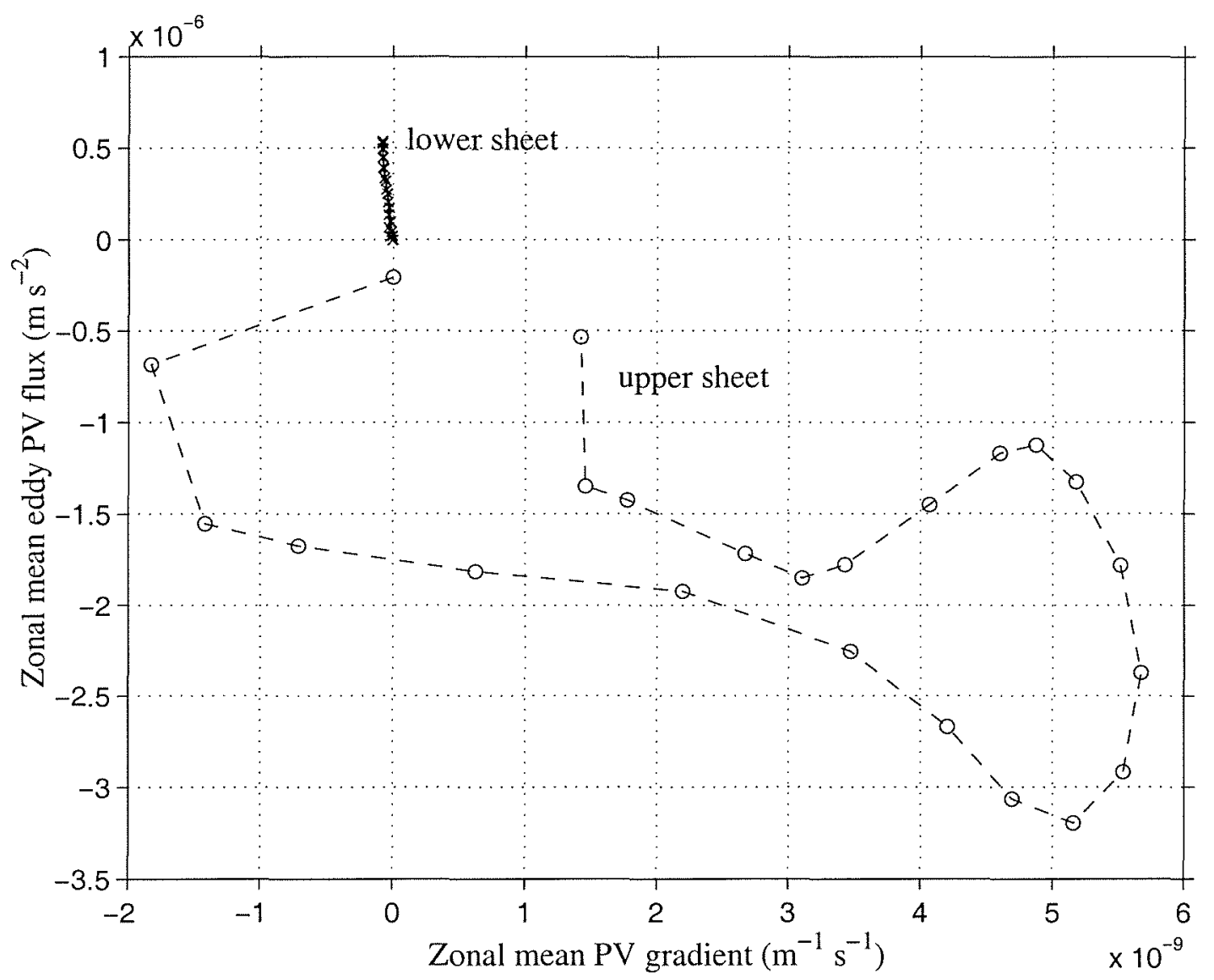

Figure 4.12: Plot of $\overline{\left(v^{\prime} q^{\prime}\right)}$ vs. $\bar{q}_{y}$ for the PV sheets. 


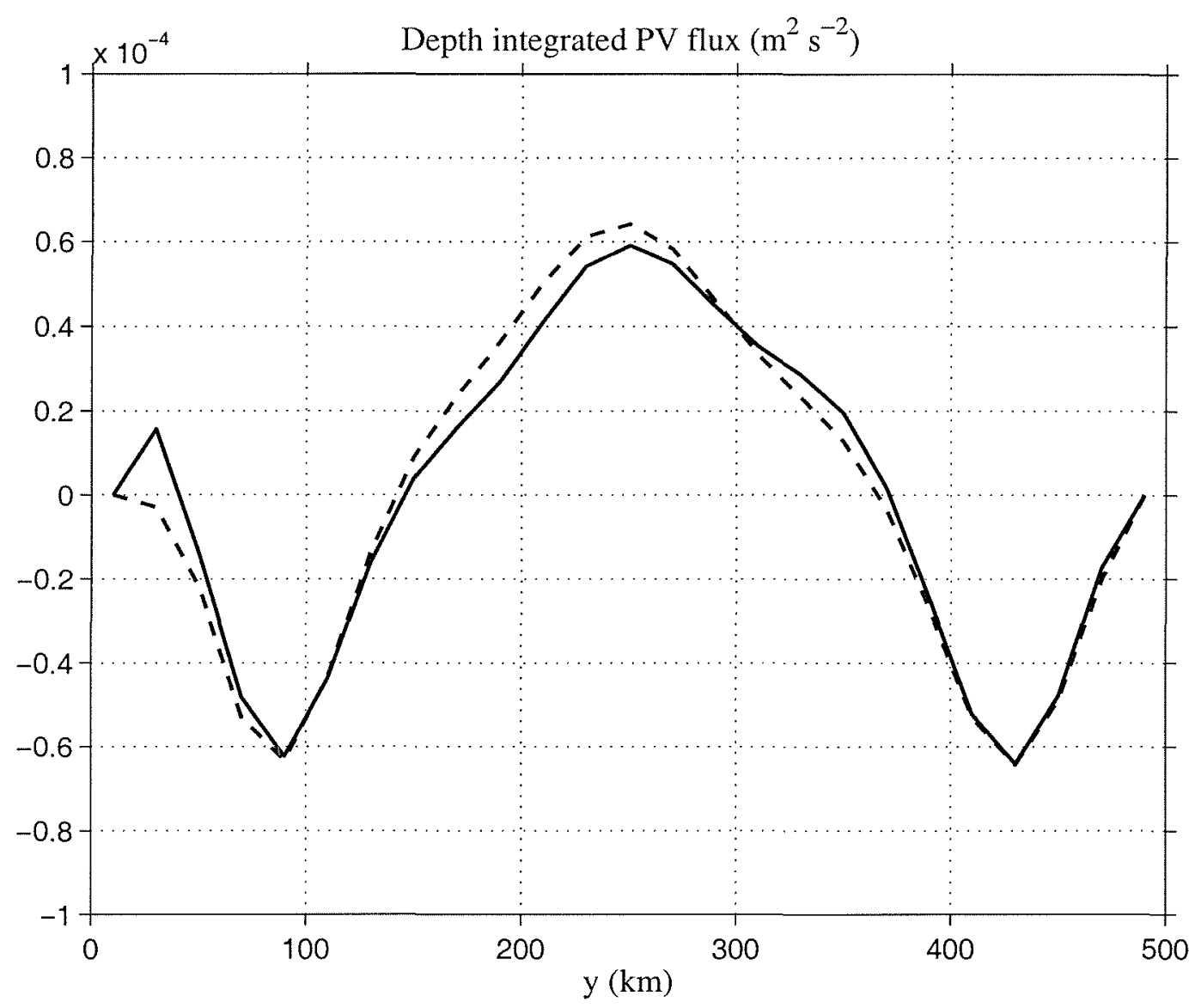

Figure 4.13: The depth-integrated Eliassen-Palm flux divergence calculated using the geostrophic streamfunction (solid line), as described in the Appendix, and from the full fields (dashed line). The very close correspondence between the two demonstrates the assumption that the eddies are quasigeostrophic in nature is an excellent for the stressdriven channel flow.

\section{How quasigeostrophic are the eddies?}

We showed in chapter 2 that if the eddies obey quasigeostrophic scaling, then their effect on the mean flow can be represented by a single term, an eddy PV flux, acting in the zonal momentum equation. Thus we now evaluate the depth-integral of this term using two different methods. In the first we use the geostrophic streamfunction and therefore geostrophic scaling; in the second we use the full primitive fields. Figure 4.13 re-plots the depth-integrated eddy potential vorticity flux $\left(\int_{-H}^{0} \overline{v^{\prime} q^{\prime}} x t d z\right)$ from figure 4.6 as a solid line. Also shown is the depth-integrated Reynolds stress divergence $\left(\int_{-H}^{0}-\overline{\left(u^{\prime} v^{\prime}\right)_{y}} x t d z\right)$ 
using the full primitive fields. The close correspondence between the two in shape and magnitude demonstrates that the quasigeostrophic scaling for the eddies is valid and correctly captures the structure and size of the eddy-forcing of the zonal mean flow.

\subsubsection{The parameterized model}

The equivalent wind-driven experiment was performed in the parameterized model (see Table 4.1). The governing equations are given by equations 2.64 .a-e, where we represent the meridional eddy flux of perturbation quasigeostrophic potential vorticity by a downgradient transfer of mean potential vorticity with the coefficient $K$ in the form expressed by equation 3.5 .

The magnitude of $\kappa_{\text {ref }}$ was chosen so that the peak of the depth-integrated transport in the zonally-averaged model matched that of the eddy-resolved calculation. As in the eddy-resolving model, the wind stress drives a southward Ekman flow in the upper level of the model which results in downward displacement of isotherms in the southern half of the channel. The meridional flow returns within the Ekman layer at the bottom level of the model inducing upward isothermal displacement to the north. This gives rise to a linearly increasing lateral temperature gradient across the channel which, through thermal wind, supports a surface-intensified jet. During this time the contribution to the quasigeostrophic PV from the relative vorticity and stretching terms increases as the flow field evolves. At each time-step the eddy PV transfer coefficient $K$ is calculated from the evolving fields using the momentum constraint equation 3.10.

Early in the evolution, the quasigeostrophic PV is everywhere dominated by the planetary vorticity gradient, $\beta$, and so the gradients of PV do not change sign and $K$ is set to zero. As the isotherms tilt, the temperature perturbations at the lower boundary give rise to a contribution to the $\mathrm{PV}$ that acts to offset $\beta$; eventually leading to a reversal in the PV gradient. Once the momentum constraint, equation 3.9, is satisfied for all $K(y, z, t)>0$ the flow satisfies conditions for baroclinic instability. At this time the $K$ 's are increased linearly with time, crudely simulating the growth of the baroclinic 
instability. The evolution of the global mean $K$ is displayed in figure 4.14.a and shows that after 5 years the model is in a steady state.
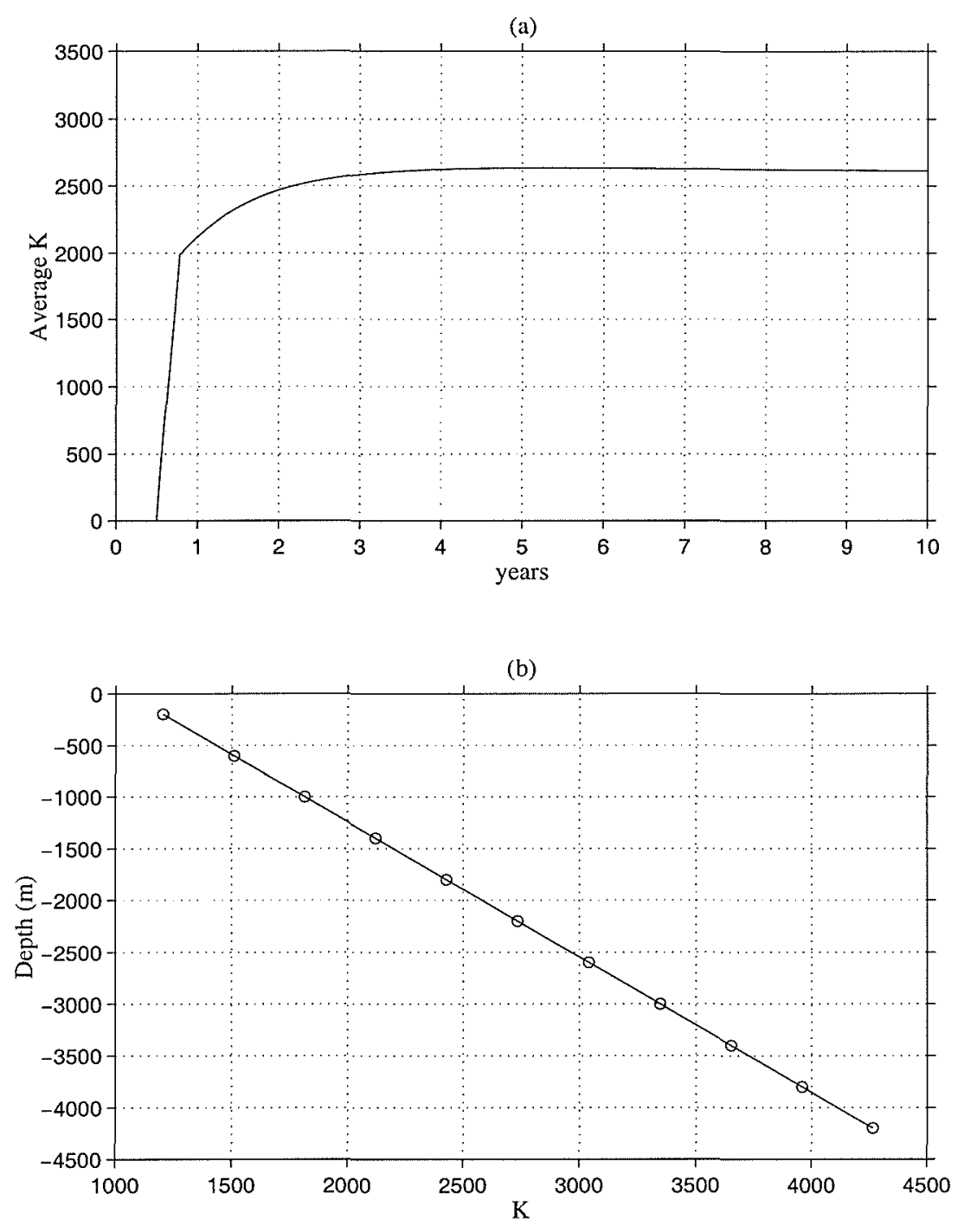

Figure 4.14: (a) The time series of the global average $K\left(\mathrm{~m}^{2} \mathrm{~s}^{-1}\right)$ in the parameterized model; (b) The steady state $K$ profile with $\kappa_{\text {ref }}=1050 \mathrm{~m}^{2} \mathrm{~s}^{-1}(\gamma=-3.21)$.

The mean zonal velocity (figure 4.15.a) consists of a surface- intensified jet in the channel center with weak return (westward flow) at depth on the flanks. The zonal velocity is in thermal wind balance with the temperature field shown in figure 4.15.b. It compares favorably with the mean flow of the resolved calculation - see figures $4.4 \mathrm{a}$, b. 
(a)

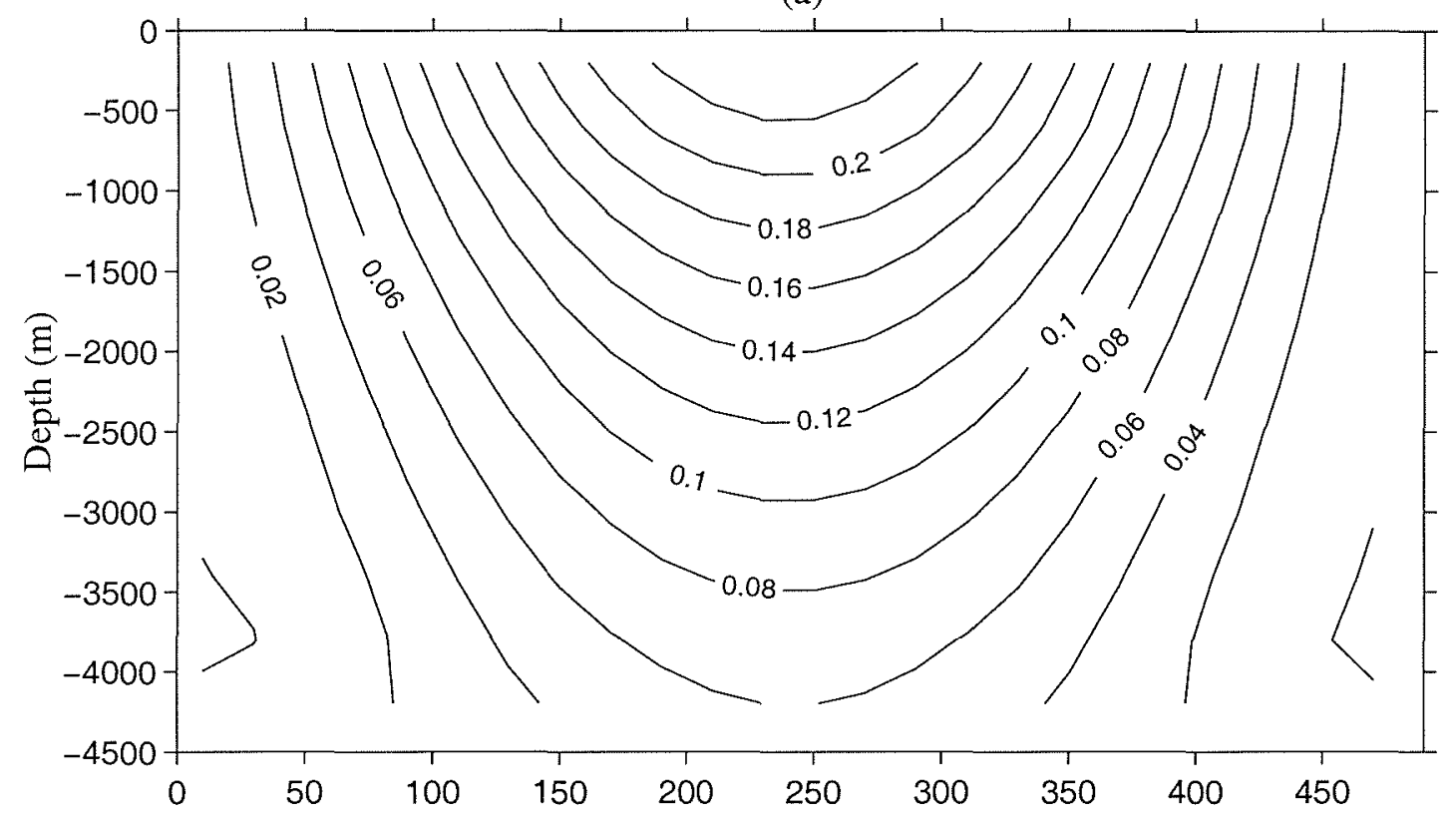

(b)

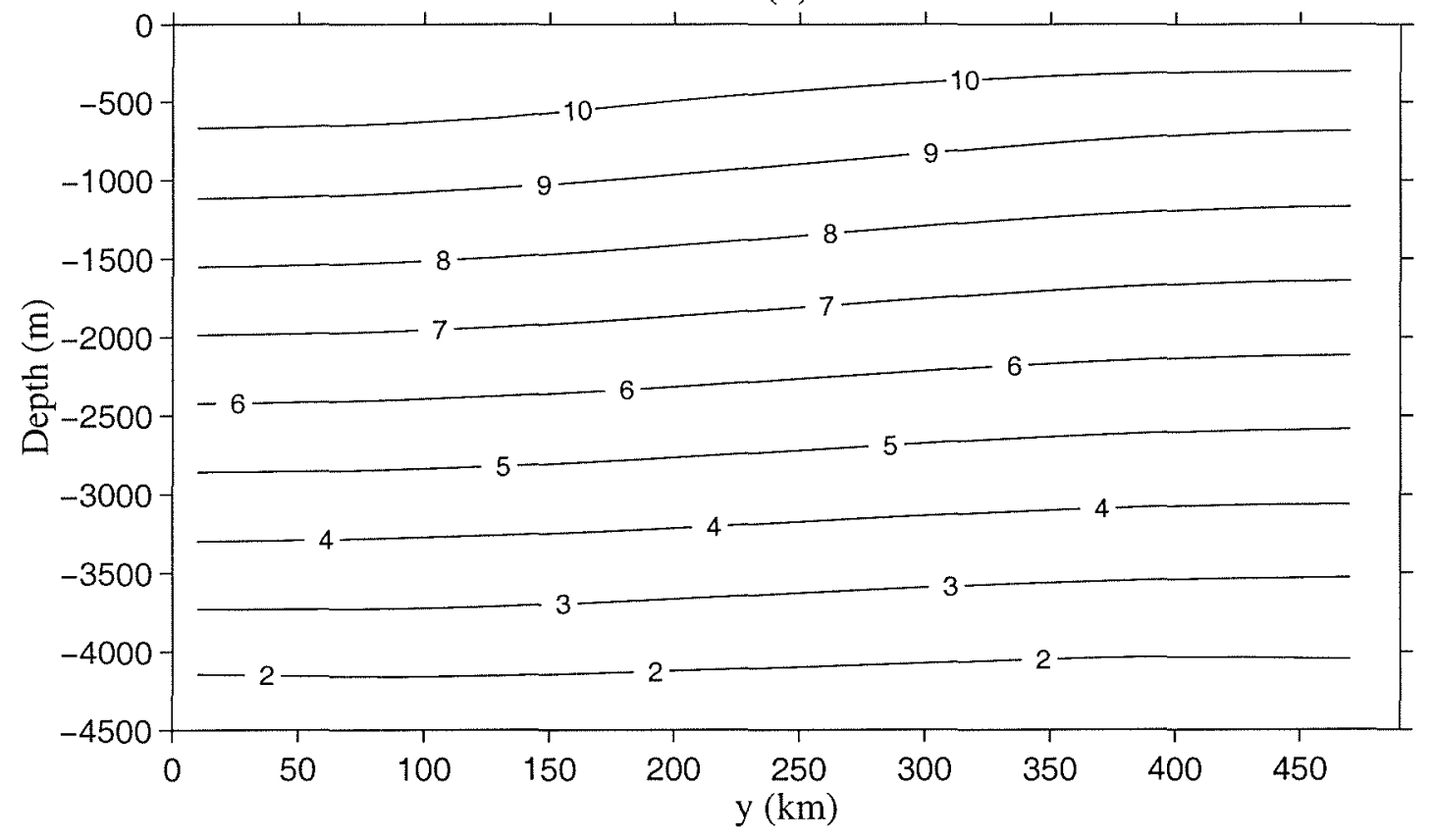

Figure 4.15: Steady state meridional cross-sections from the parameterized model: (a) zonal mean zonal velocity $\left(m s^{-1}\right)$; (b) zonal mean temperature. 
The steady state zonal momentum balance throughout the fluid is

$$
0=\bar{F}_{x}+{\overline{v^{\prime} q^{\prime}}}^{x} .
$$

The balances in equation 4.13) are shown in figure 4.16. At the upper boundary (figure 4.16.a) the wind stress is balanced by the term representing the eddy flux of quasigeostrophic potential vorticity giving a sheet with a southward eddy flux of potential vorticity. At the bottom (figure 4.16.c) the stress is balanced by the parameterized terms representing a northward eddy flux of potential vorticity. In the interior (figure 4.16.b), there is no applied force, thus in the steady state the eddy PV flux is zero. Because of the flux gradient relationship assumed for the eddy PV flux (equation 3.5), this means that the interior PV gradients are zero. This compares favorably to the eddy-resolving model where interior PV gradients, away from the side wall, are small when compared to $\beta$.

The reference transfer coefficient in the upper layer was specified to be $1050 \mathrm{~m}^{2} \mathrm{~s}^{-1}$. In the steady state the value of the free parameter $\gamma$ was -3.21 , giving

$$
K=1050\left(1-3.21 \frac{z}{H}\right) \quad m^{2} s^{-1} .
$$

Figure 4.14.b shows the $K$ profile. The transfer coefficient becomes large in the lower PV- sheet to compensate for the small negative potential vorticity gradient there, just as for the $K^{\prime} s$ diagnosed from the resolved model. As shown in figure 4.16 the steady state $\overline{v^{\prime} q^{\prime}}$ consists of boundary sheets with divergence at the lower horizontal boundary and convergence at the surface. The meridional profile is shown in figure 4.17.a. This is consistent with the PV flux signature for the eddy resolving calculation. The depthintegrated parameterized eddy PV flux is plotted in figure 4.17.b and shows that the effect of the eddies is to exert a positive (eastward) body force on the zonal momentum in the center of the jet and a negative (westward) body force on the flanks. Thus momentum is transferred upgradient into the jet center resulting in the depth integrated zonal flow shown in figure 4.18. This agrees with diagnosed eddy-forcing of the zonal mean flow 

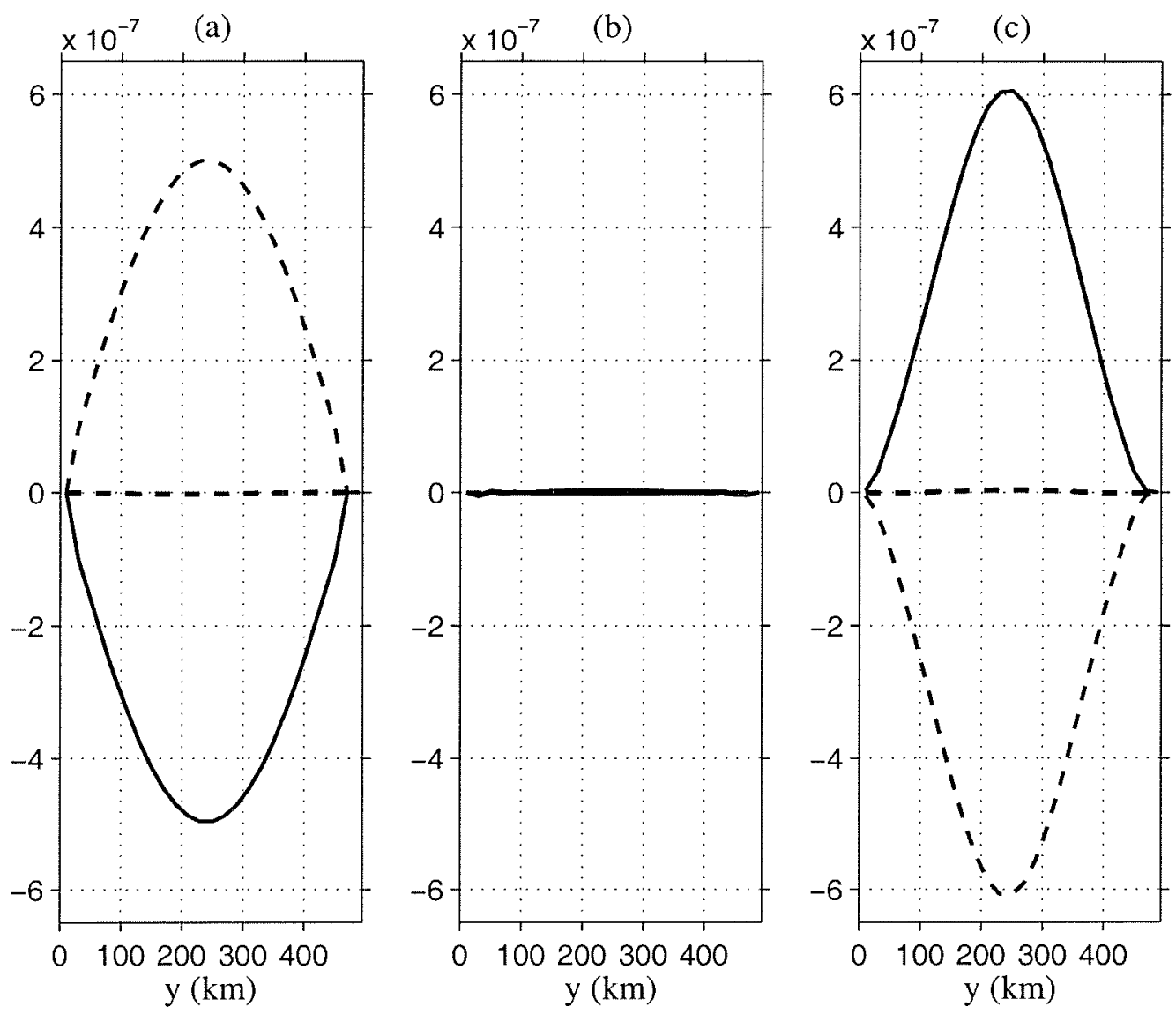

Figure 4.16: The stress (dashed) and eddy flux of quasigeostrophic potential vorticity (solid) terms in the steady state momentum equation for; (a) the upper layer; (b) the interior; (c) the lower layer. Equation 4.13 is exactly satisfied.

from the eddy resolving flow and demonstrates that the zonal average model can capture this rather subtle aspect of eddy-mean-flow interaction.

One shortcoming of the zonal average model is that it fails to take into account some of the nonlocal effects. The resolved fields exhibit a change in sign of the surface quasigeostrophic potential vorticity close to the southern vertical wall which is absent from the parameterized model. A second difference is that the magnitude of the parameterized depth-integrated eddy PV flux is $25 \%$ less than that diagnosed from the eddy resolving calculation. The size of the potential vorticity flux in the upper sheet in each 

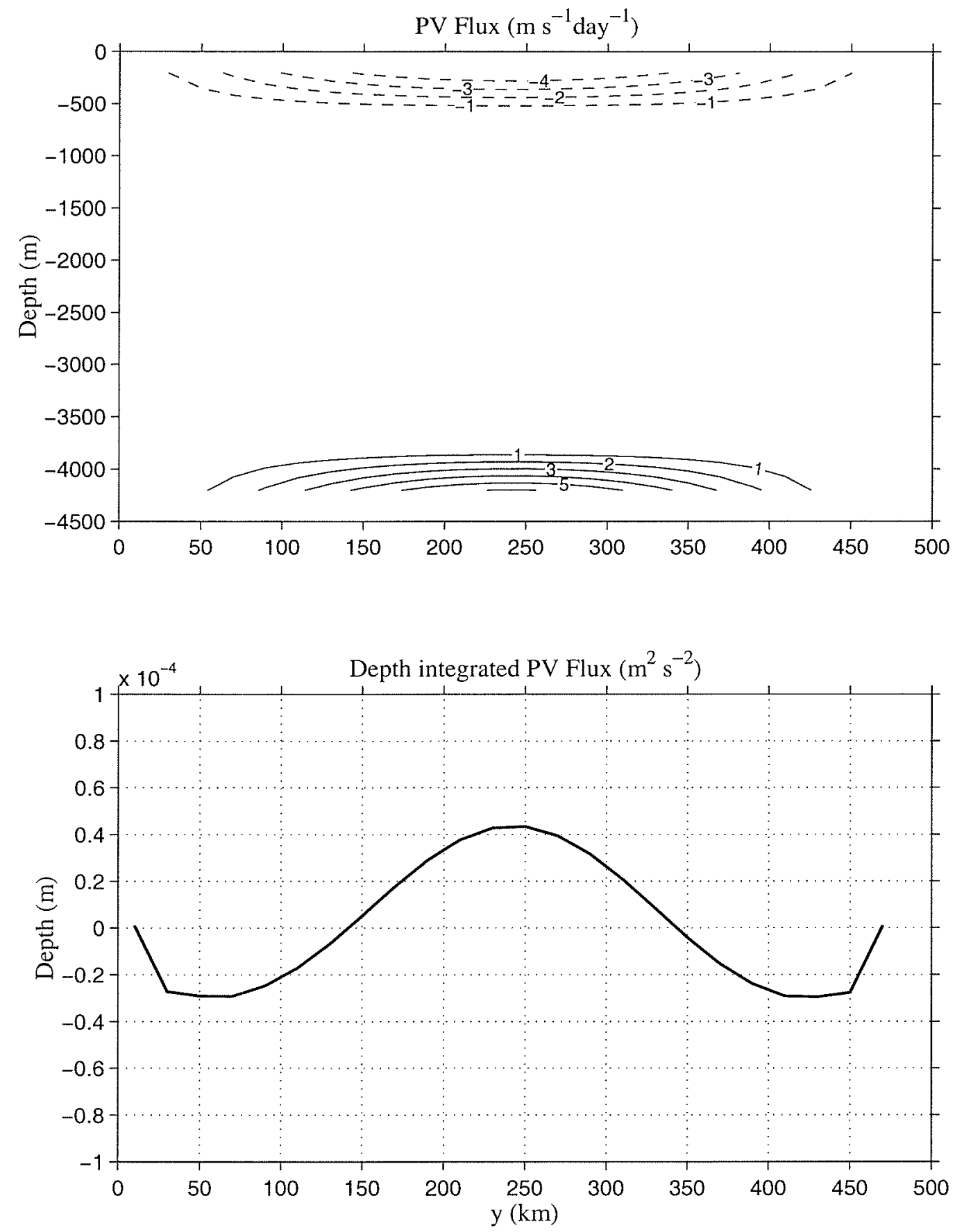

Figure 4.17: (a) The PV flux $\left(\overline{v^{\prime} q^{\prime}}\right)$ is concentrated at the boundaries; (b) The depth integrated PV flux. Its effect is to exert a positive (eastward) body force on the zonal momentum in the center of the channel, and a negative (westward) body force in the flanks of the jet, consistent with the eddy characteristics diagnosed from the eddy-resolving model. 

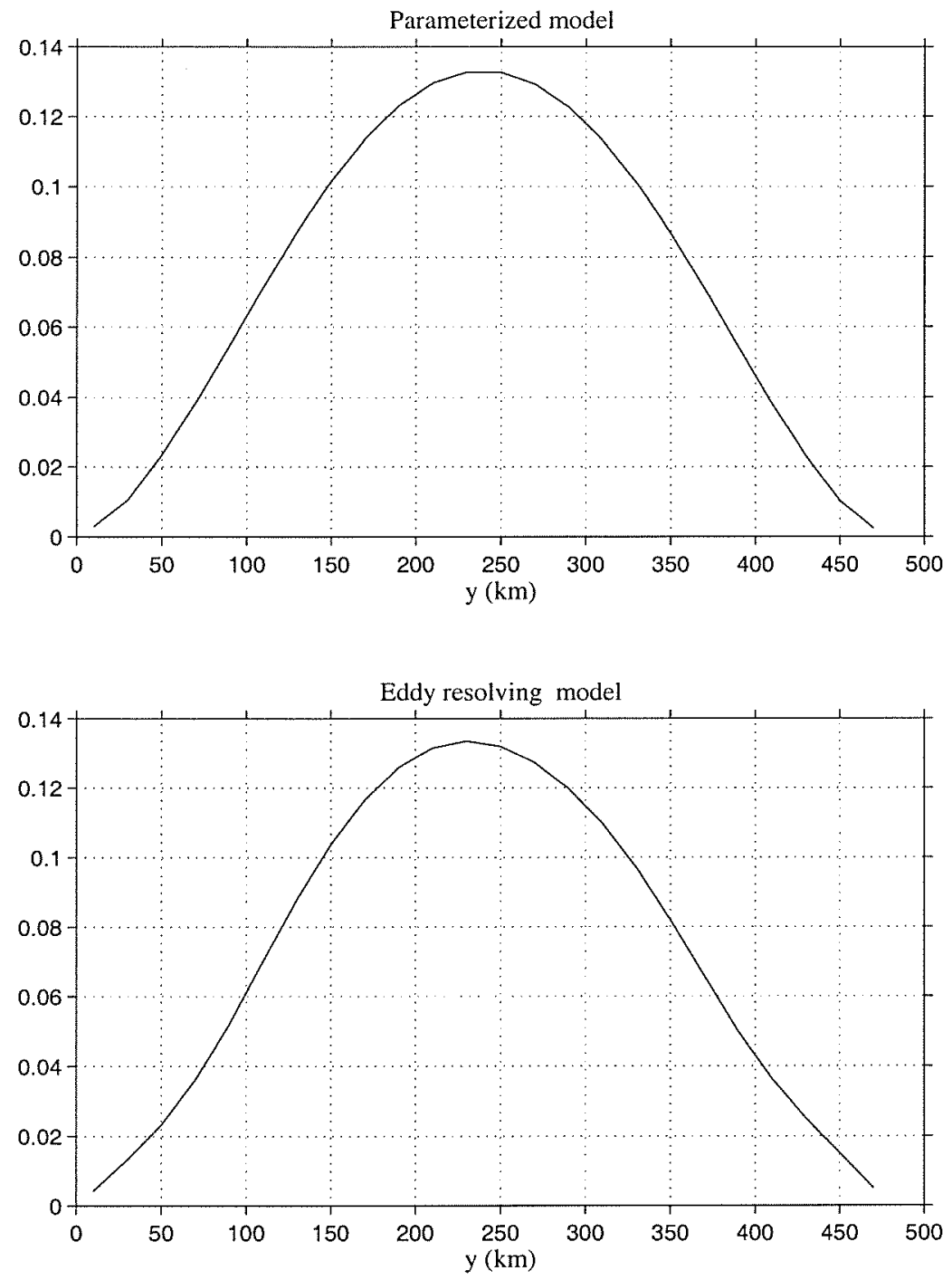

Figure 4.18: The depth average of the zonal mean zonal velocity in the (a) parameterized model; (b) eddy-resolving model.

model is very similar (it has to be because through equation 4.13 it has to balance the surface-stress in each model), but the magnitude of the positive potential vorticity flux is underestimated in the parameterized model. Since this flux acts to balance the bottom drag, the velocities at depth in mid-channel are smaller for the parameterized model as can be seen by comparing figures 4.4.a and 4.15.a, even though the depth-integrated zonal mean flow are very similar. Consequently the depth integral of this potential vorticity 
flux is smaller for the parameterized case.

Despite these differences, the parametrically represented eddy transfer of buoyancy and momentum display the same characteristics as the eddy resolving calculation and as a result the zonal mean fields are in very good agreement.

\subsection{Spin-down of a baroclinic zone on a $\beta$-plane}

We now consider, following Gent et al. (1995) and Visbeck et al. (1996), the spin-down of a baroclinic zone in the absence of external forces, apart from a bottom drag at depth. Again, we compare calculations from the three-dimensional numerical model that resolves the baroclinic eddy field to the zonal average model where we parameterize the eddy PV transfer.

\subsubsection{The eddy-resolving model}

The sloping zone is characterized by meridional temperature profile displayed in figure 4.19. The initial stratification is of constant value in the vertical. The slope is uniform

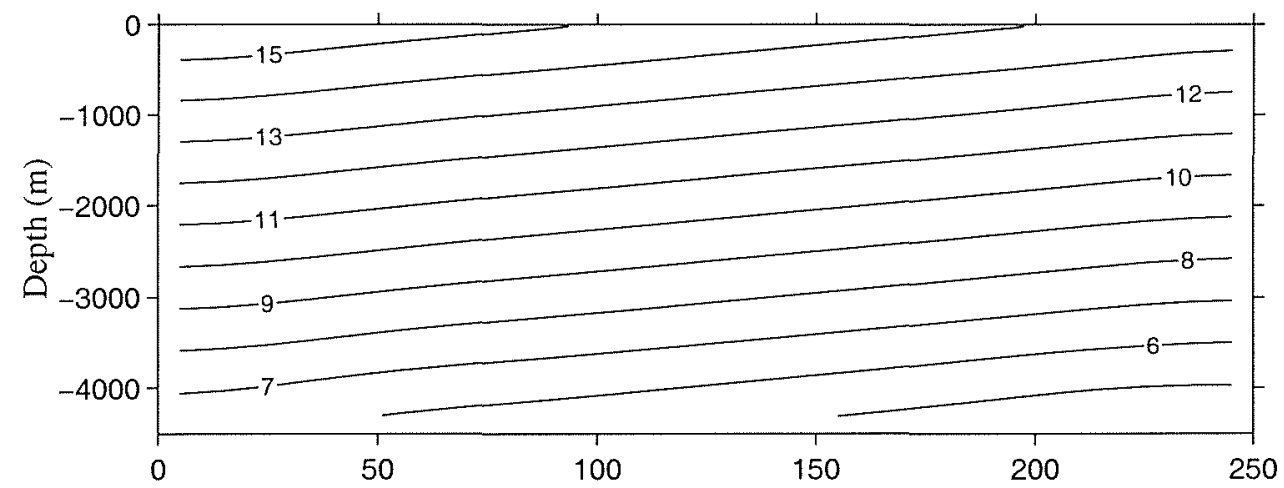

Figure 4.19: Spin-down of a baroclinic zone. The initial temperature cross-section. 
in the y direction except at the walls where the isotherms flatten. The isotherms surface throughout the surface of the channel and ground at the lower boundary. The model has twenty active levels in a periodic channel of length $750 \mathrm{~km}$, width $250 \mathrm{~km}$, and depth 4500 $m$ and was integrated for 10 years (see Table 4.2). The time evolution of the instability

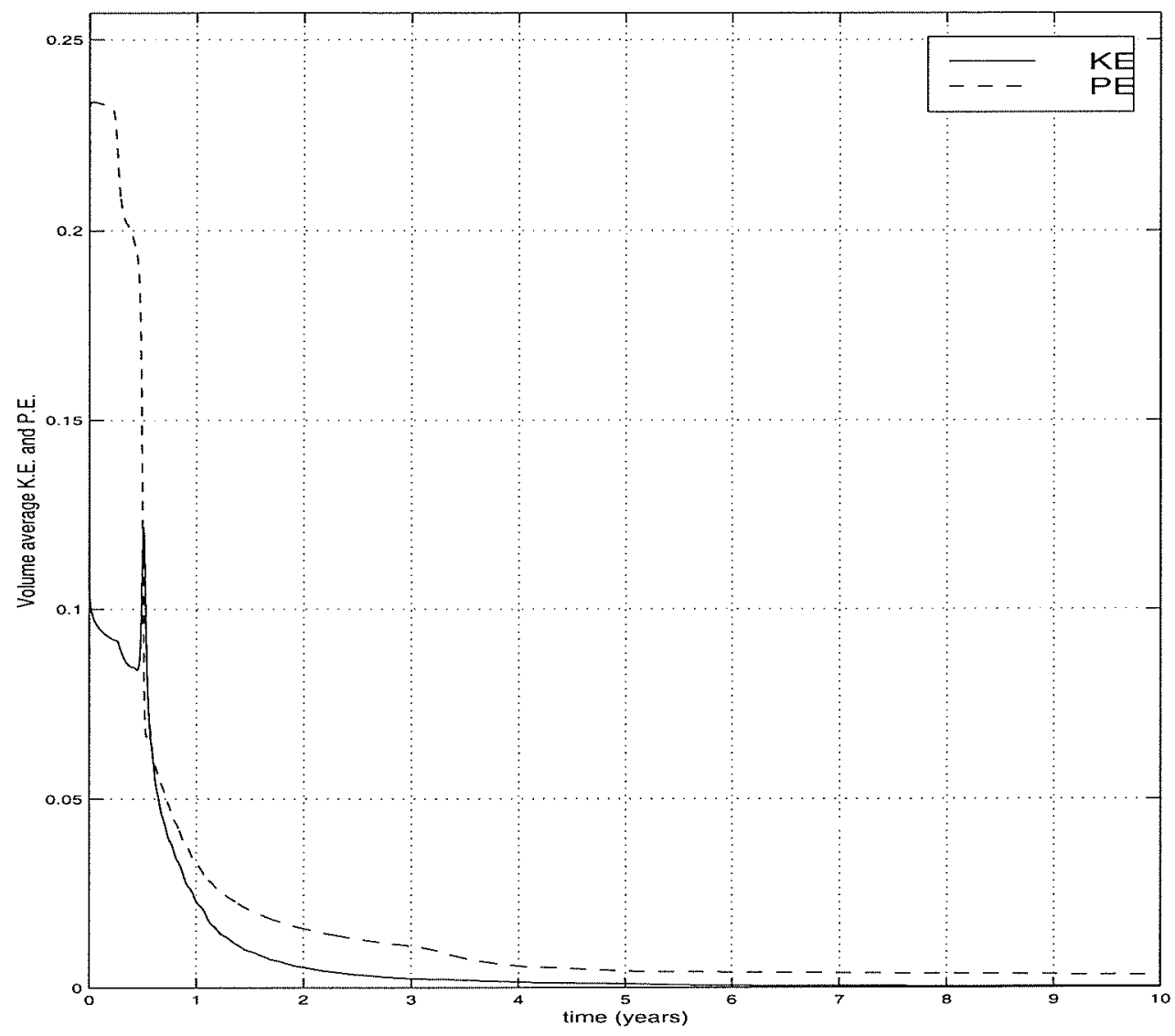

Figure 4.20: Spin-down of a baroclinic zone. The times series of the available potential energy and kinetic energy per unit mass per unit volume.

is summarized in figure 4.21 which shows surface temperature and velocity fields at time $=165,180,240$, and 3600 days. The velocity field is quivered at every other grid-point, with the scale for the first three panels being equal and the fourth panel (3600 days) being multiplied by a factor of three. Initially, the along channel velocity has maxima of approximately $0.9 \mathrm{~ms}^{-1}$. The surface temperature is colored and contoured and shows 


\begin{tabular}{|c|c|c|c|}
\hline Parameter & units & Eddy-resolving model & Parameterized model \\
\hline$f_{0}$ & $s^{-1}$ & $1 \times 10^{-4}$ & $1 \times 10^{-4}$ \\
\hline Bottom drag & $s^{-1}$ & $1 \times 10^{-5}$ & $1 \times 10^{-5}$ \\
\hline $\mathrm{x}$-domain & $\mathrm{km}$ & 750 & - \\
\hline $\mathrm{y}$-domain & $\mathrm{km}$ & 250 & 250 \\
\hline Depth & $\mathrm{m}$ & 4500 & 4500 \\
\hline Horizontal grid size & $\mathrm{km}$ & 10 & 10 \\
\hline Vertical grid size & $\mathrm{m}$ & $50-400$ & $50-400$ \\
\hline Vertical levels & & 21 & 21 \\
\hline Initial stratification $\left(N / f_{0}\right)$ & & 21 & 21 \\
\hline Rossby radius $\left(\mathrm{NH} / f_{0}\right)$ & $\mathrm{km}$ & 95 & 95 \\
\hline Horizontal diffusivity & $m^{2} s^{-1}$ & 0 & 0 \\
\hline Biharmonic diffusivity & $m^{4} s^{-1}$ & 0 & 0 \\
\hline Vertical diffusivity & $m^{2} s^{-1}$ & 0 & 0 \\
\hline Horizontal viscosity & $m^{2} s^{-1}$ & 0 & 0 \\
\hline Biharmonic viscosity & $m^{4} s^{-1}$ & $2 \times 10^{10}$ & $2 \times 10^{10}$ \\
\hline Vertical viscosity & $m^{2} s^{-1}$ & 0 & 0 \\
\hline$K_{p v}$ & $m^{2} s^{-1}$ & - & 15 \\
\hline $\mathrm{T}(\mathrm{t})$ & & - & linear ramp: 100 days \\
\hline$Y(y)$ & & - & $0, y=0, L_{y}$ \\
\hline & & - & $1,0<y<L_{y}$ \\
\hline
\end{tabular}

Table 4.2: Parameters for the eddy-resolving and parameterized spin-down experiments. 
the outcrops of the temperature field. The front becomes baroclinically unstable after approximately 165 days. By day 180 finite amplitude eddies fill the channel which are organized to give a cross-zone ageostrophic flow which transfers fluid from one side of the channel to the other. In the northern half of the channel downwelling pumps cold water down, while to the south the isotherms are raised. This results in a release of the mean potential energy stored in the sloping density surfaces. The time series of average available potential energy and kinetic energy is shown in figure 4.20 . It shows that a statistically steady state is reached after 6 years of integration and the time-average was obtained from the last three years of model time. Averaging the three-dimensional fields along the front in the equilibrated state state yields a surface intensified jet with along front peak velocity of $0.144 \mathrm{~ms}^{-1}$ in the center of the channel (see figure 4.22.b.c). The jet is in thermal wind balance with the temperature field shown in figure $4.22 \mathrm{~b}$. The final state is not one of zero flow because of the stabilizing effect of the planetary PV gradient, $\beta$. 

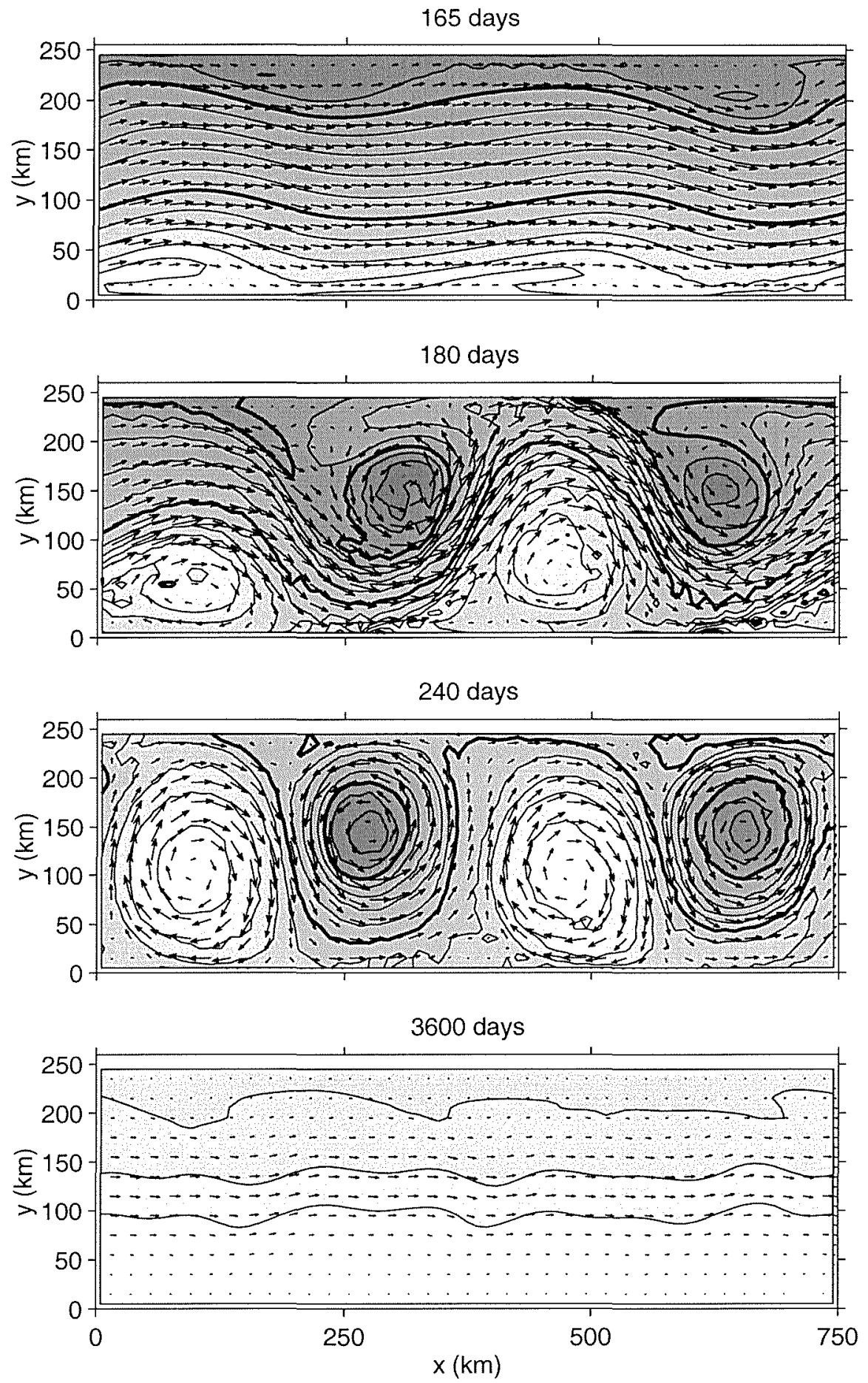

Figure 4.21: Spin-down of a baroclinic zone. Surface temperature and velocities from the eddy-resolving channel model after 165, 180, 240 and 3600 days. The temperature is contoured and shaded with lighter shading denoting warmer water. 
(a)

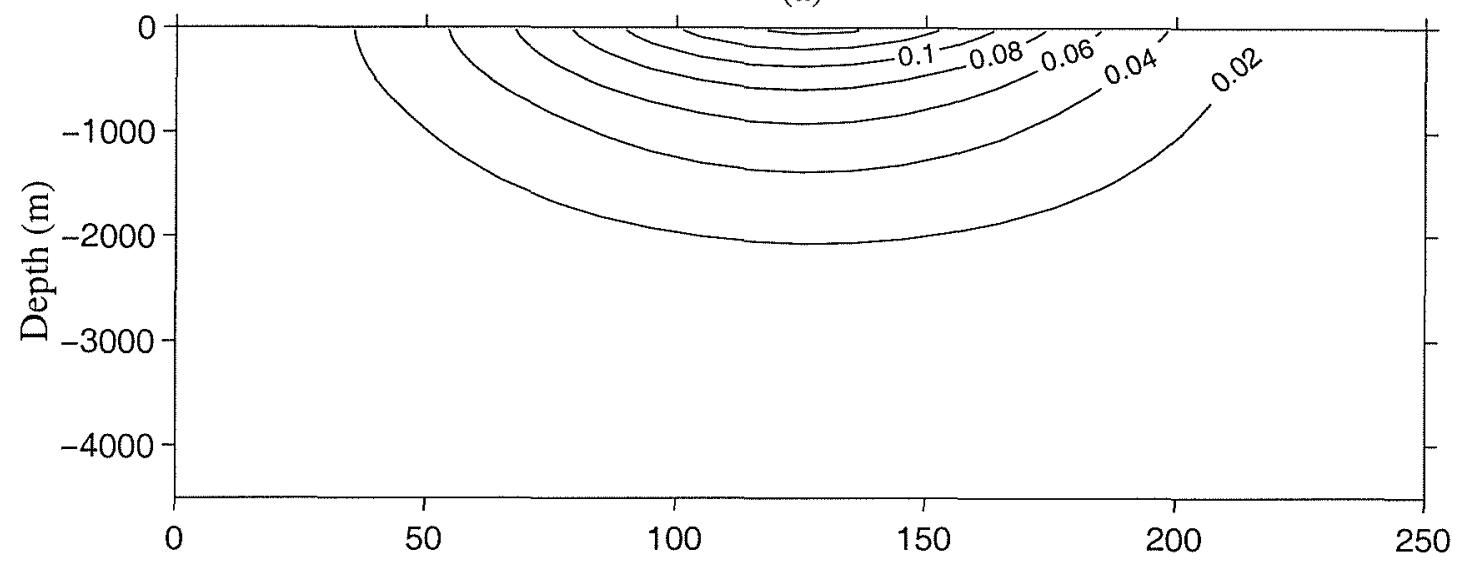

(b)

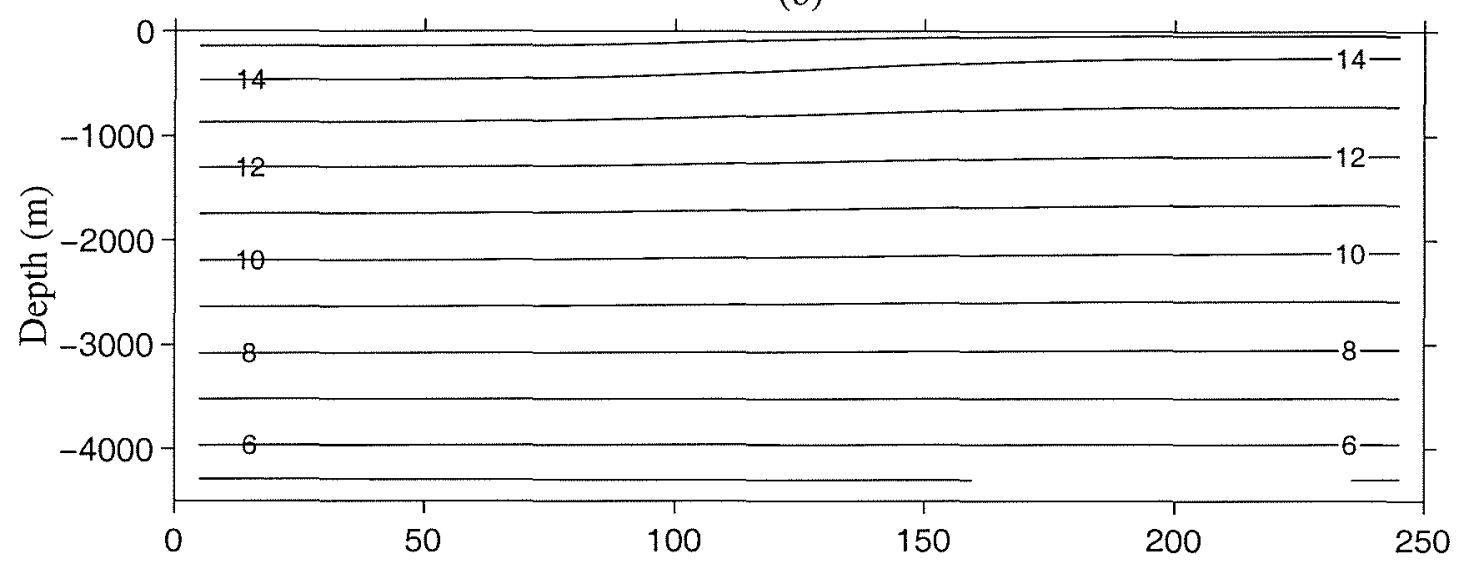

(c)

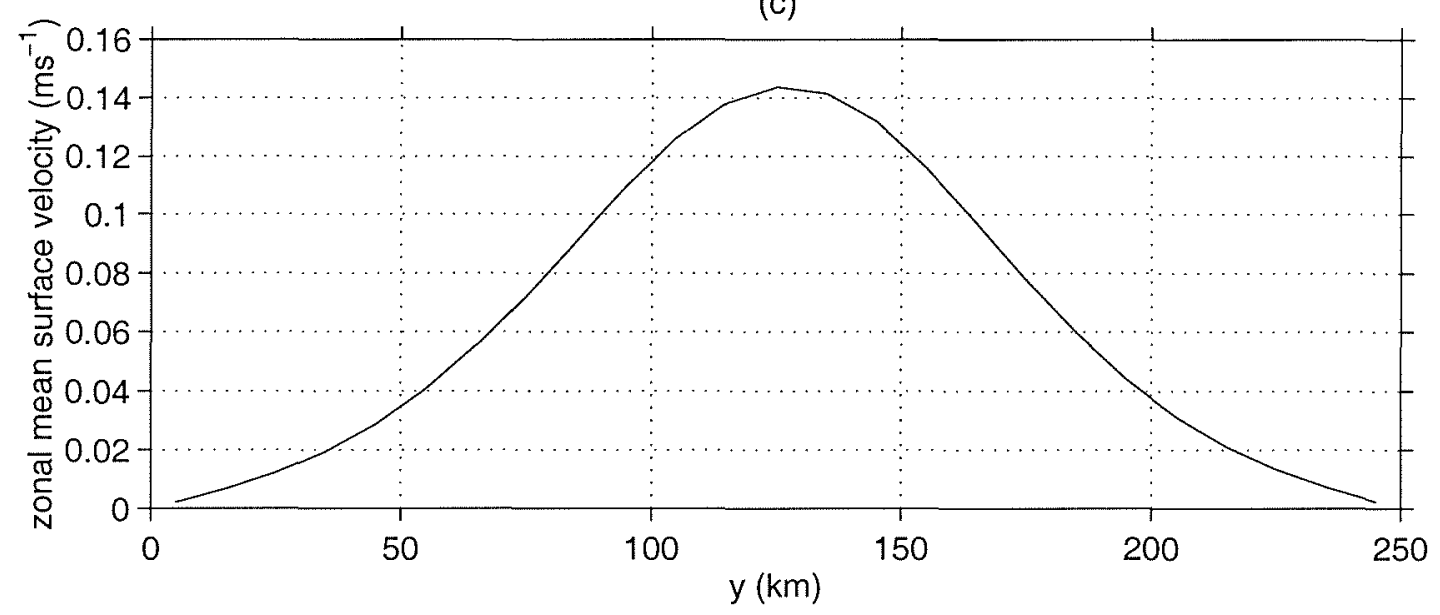

Figure 4.22: Spin-down of a baroclinic zone. Zonal average fields from the eddy resolving model. The time-averaged meridional cross-sections of: (a) zonal mean zonal velocity $\left(m s^{-1}\right)$; (b) zonal mean temperature; (c) zonal mean surface velocity $\left(m s^{-1}\right)$. The time average was taken over the last three years of integration. 


\subsubsection{The parameterized model}

The 2D model was employed for the same problem and was initialized with the same meridional temperature profile. Unlike in section 4.2 , here the eddies and ther parameterized fluxes are only present in the transient stage of flow. However, the final state depends explicitly on the eddy transfers in the transient phase.

The PV gradients in the interior are essentially the planetary vorticity gradient, $\beta$, with relative vorticity contributing as the side walls are approached. To the south at all depths the fluid is warmer than if the isotherms were horizontal, while to the north the temperatures are cooler. The attendant temperature perturbations along the upper and lower boundaries are associated with PV gradient sheets which oppose one another and satisfy the necessary conditions for baroclinic instability.

The evolution of the zonally-averaged flow closely obeys the following momentum balances:

$$
\begin{aligned}
\bar{u}_{t}-f \bar{v}^{\star} & =\overline{v^{\prime} q^{\prime}} \\
\bar{u}_{t}-f \bar{v}^{\star} & =\overline{v^{\prime} q^{\prime}} \\
\bar{u}_{t}-f \bar{v}^{\star} & =\overline{v^{\prime} q^{\prime}}-\epsilon \bar{u}
\end{aligned}
$$

for the upper, interior, and lower regions respectively. In the model that the primary momentum balance is between the Coriolis and eddy flux terms with the zonal momentum tendency being the residual between the two. The meridional velocity is northward in the upper sheet enabling the Coriolis term to balance the meridional potential vorticity flux. In the lower sheet the zonal momentum tendency is the residual of the balance between the Coriolis, eddy-flux and bottom-drag terms. The meridional velocity is southward in this sheet. In the interior the zonal mean flow is accelerated by the residual between Coriolis forces and eddy PV forcing. The residual mean circulation draws the warmer water in the south upward, and the colder water to the north downward, releasing available potential energy and spinning down the zone. This continues until the component of the PV gradients associated with the temperature perturbations of the sheets at the lower 
(a)

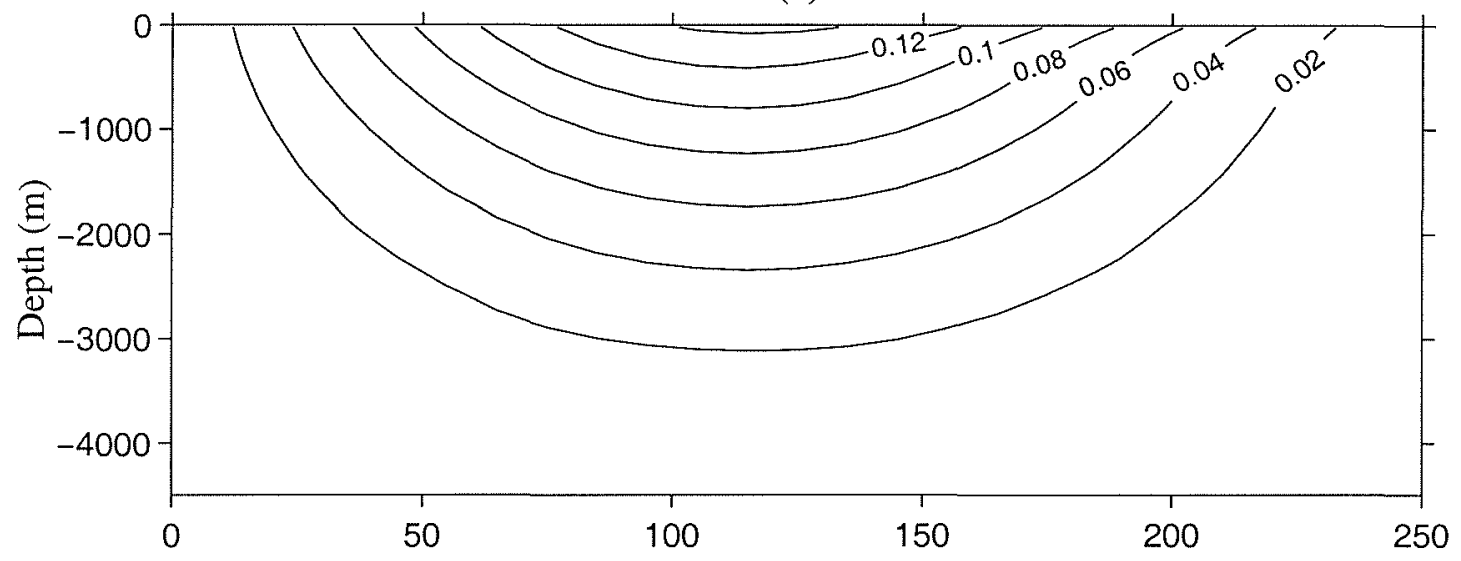

(b)

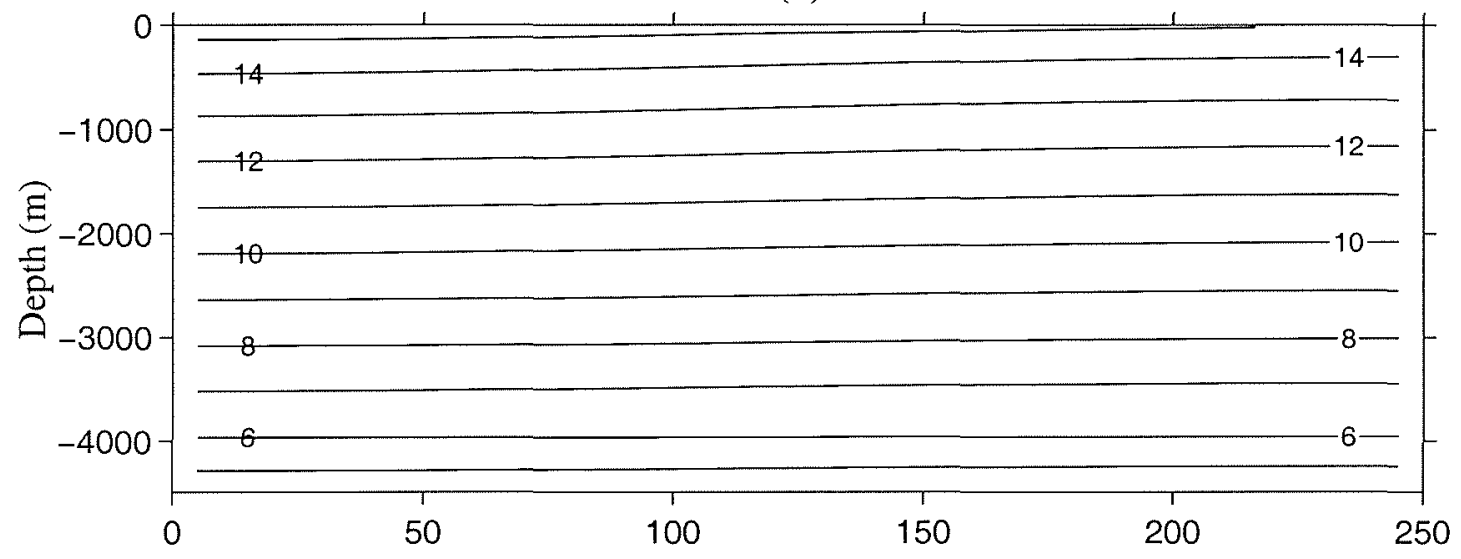

(c)

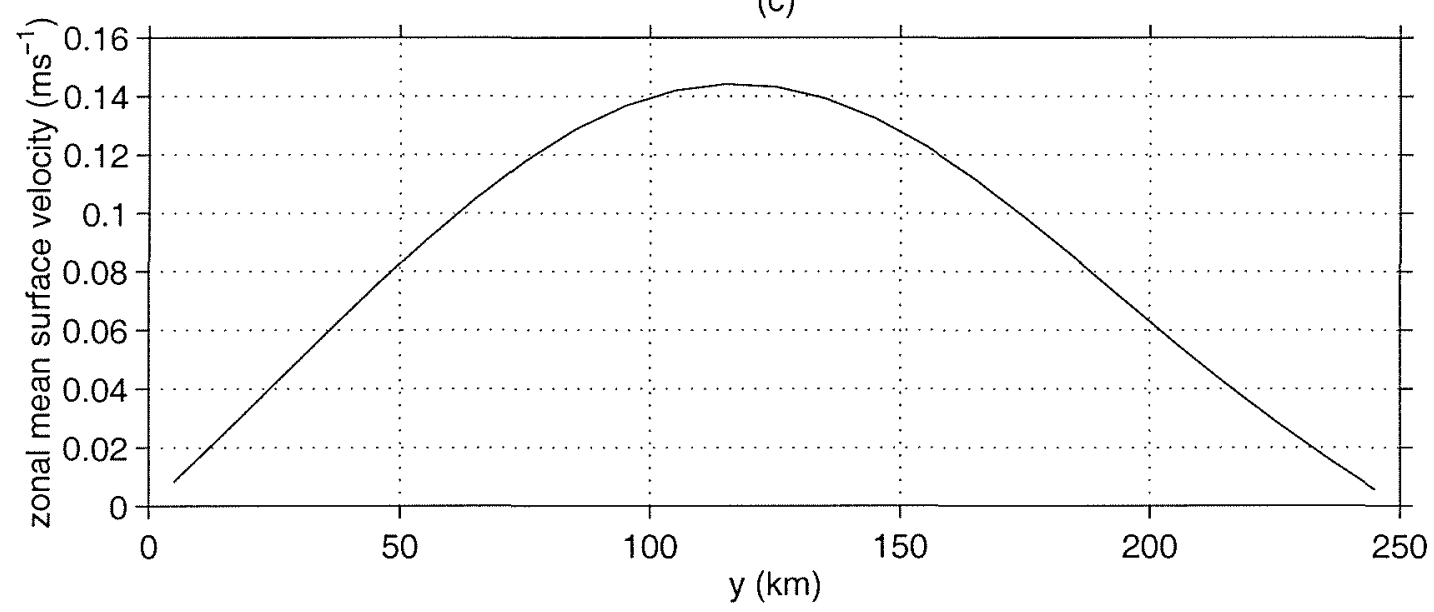

Figure 4.23: Spin-down of a baroclinic zone. Zonal average fields from the parameterized model. The final-state meridional cross-sections of: (a) zonal mean zonal velocity $\left(\mathrm{ms}^{-1}\right)$; (b) zonal mean temperature; (c) zonal mean surface velocity $\left(\mathrm{ms}^{-1}\right)$. 
boundary are too weak to offset $\beta$. At this point the necessary conditions for baroclinic instability are no longer satisfied and further spin-down ceases due to the stabilizing effect of the planetary vorticity gradient.

In the parameterized model, if the gradients of potential vorticity do not change sign; the momentum constraint, equation 3.9, can only be satisfied if the transfer coefficient takes on both signs in the flow. When this occurs, $K$ is set to zero and the eddy driving term vanishes. The equilibrium state is one in which there is a sloping zone (figure 4.23.b) with a mean zonal jet (figure 4.23.a) in thermal wind balance. The parameterized eddies have released the potential energy stored in the mean flow. The peak velocity at the surface is $0.142 \mathrm{~ms}^{-1}$, similar to the along-zone maximum found in the eddy resolving calculation. However, In contrast to the eddy resolving run we have stronger flows at depth, as can be seen by comparing figures 4.22 and 4.23 . Thus the depth integrated flow is overestimated in the parameterized model.

The limiting case of section 2.8.2 is now employed in the zonal average model. Relative vorticity fluxes are set to zero in equation 2.15 and so equation 3.9 is automatically satisfied. A constant value of $K$ is used, as in GM. The initial fields satisfy the necessary conditions for baroclinic instability and so the evolution of the flow proceeds as before. However, as the zone spins down and the gradients of the temperature perturbation on each boundary decrease because there is no stabilizing absolute vorticity gradient. Thus the potential vorticity gradients at the upper and lower boundaries take on opposite sign whenever boundary temperature perturbations exist. Thus the $\beta$-stabilization present when the relative vorticity fluxes are included does not occur here. The final state of the zone is shown in figure 4.24, where the zero relative vorticity flux limit of our parameterization (equivalent to GM) has adiabatically flattened the isotherms until the zone is horizontal with no zonal flow. 
(a)

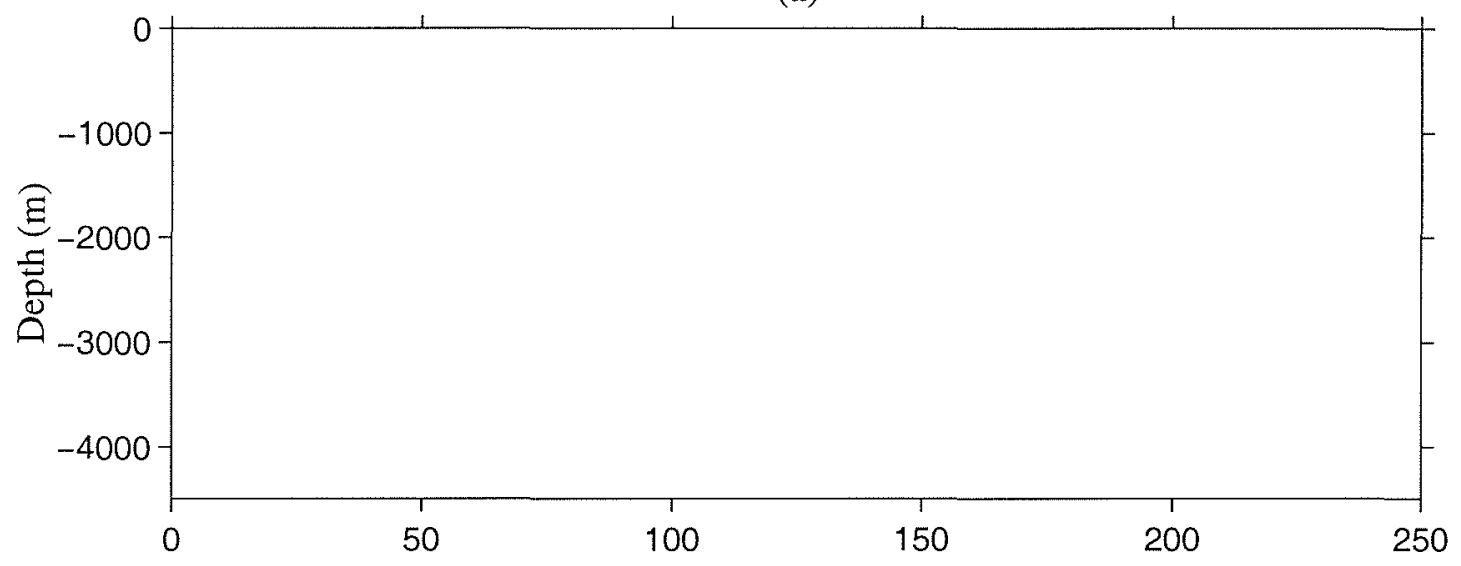

(b)

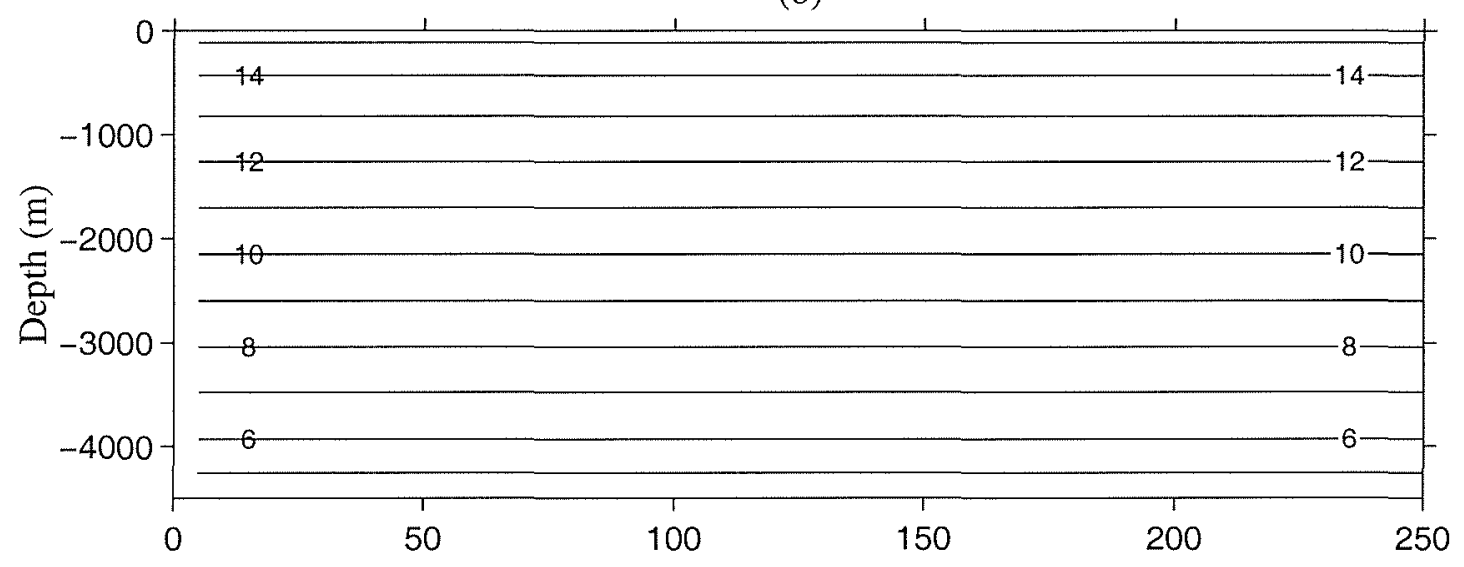

(c)

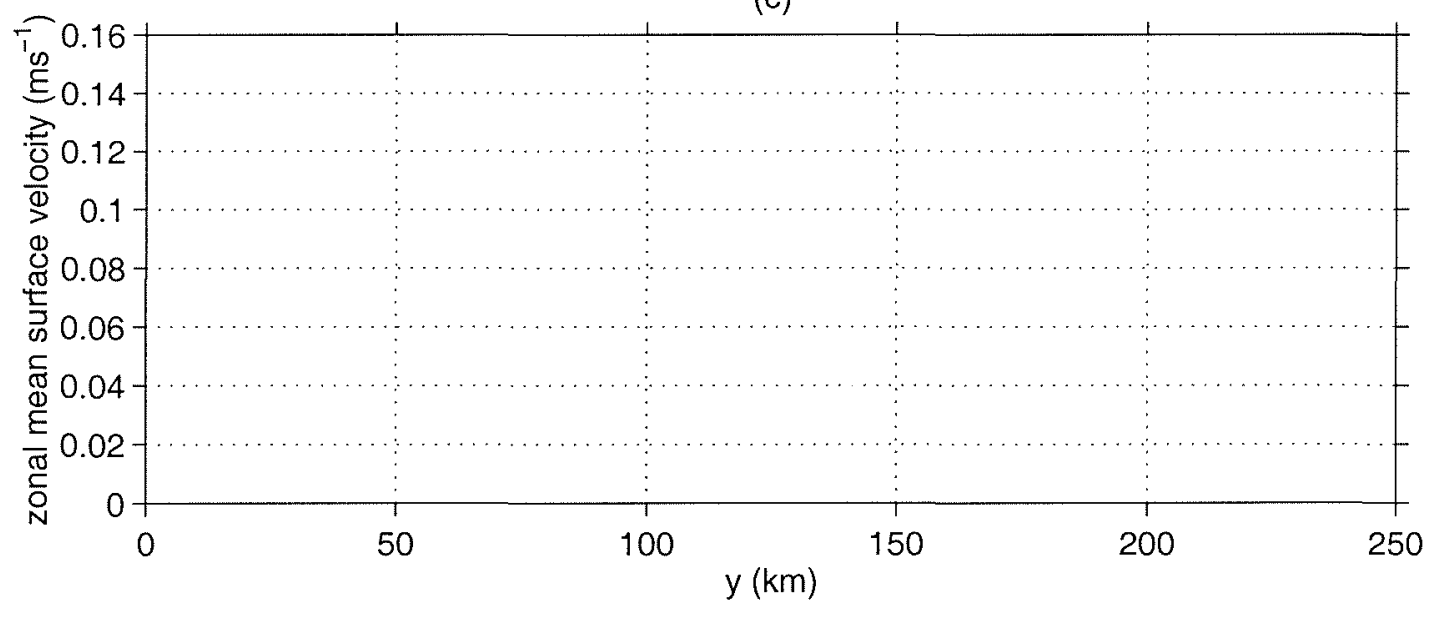

Figure 4.24: Spin-down of a baroclinic zone. Zonal average fields from the parameterized model when the relative vorticity fluxes are ignored. The final-state meridional crosssections of: (a) zonal mean zonal velocity $\left(\mathrm{ms}^{-1}\right)$; (b) zonal mean temperature; (c) zonal mean surface velocity $\left(m s^{-1}\right)$. 


\subsection{Tropospheric eddies in the atmosphere}

The troposphere provides a very interesting test of the theoretical ideas outlined in sections 2 and 3 because baroclinic eddies are the most important component of the atmospheric general circulation outside of the tropics [Jeffreys (1926); Starr (1948); Lorenz (1967)]. The net radiative budget of the Earth-atmosphere system, averaged over a year, results in a net surplus of incoming radiation in the tropics and a net deficit at high latitudes. Thus for the global climate to be in equilibrium there must be transport of energy from low to high latitudes, in order to balance the terrestrial radiative budget. Extratropical transport occurs through motions generated by the baroclinic instability of the mid-latitude zonal flow. But the instability also helps maintain the zonal mean through both the eddy heat and momentum fluxes.

We present three experiments here with a zonally-averaged TEM aatmospheric model: (i) no eddy-forcing, (ii) eddy-PV-forcing, and (iii) eddy-PV-forcing in the absence of relative vorticity fluxes. The model solves the governing equations for an ideal gas atmosphere in hydrostatic balance. The hydrodynamical core is that of the MIT ocean model, but we employ isomorphisms to yield a $p$-coordinate model applicable to the flow of a compressible atmosphere - see Appendix A. Potential temperature, $\theta$, replaces $T$ in the thermodynamic equation, (equation 2.64.d). Forcing is through relaxation of $\theta$ to a prescribed "radiative equilibrium" temperature $\bar{\theta}_{e q}(p, y)$ on a timescale $\tau(p, y)$ which are both functions of pressure and latitude (Held and Suarez, 1994). Thus the potential temperature equation takes the form:

$$
\bar{\theta}_{t}+\bar{v}^{\star} \bar{\theta}_{y}+\bar{w}^{\star} \bar{\theta}_{p}=-\frac{1}{\tau}\left(\bar{\theta}-\bar{\theta}_{e q}\right) .
$$

Surface drag is represented through a quadratic drag law and there is no orography.

Five model levels are used, the lowest being at $950 \mathrm{mb}$, at the top of the surface boundary layer, and the highest at $75 \mathrm{mb}$, in the stratosphere. The parameters used in these experiments are summarized in Table 4.3. The initial state is a horizontally stratified atmosphere as shown in figure 4.25.a which is then relaxed to the prescribed 
(a)

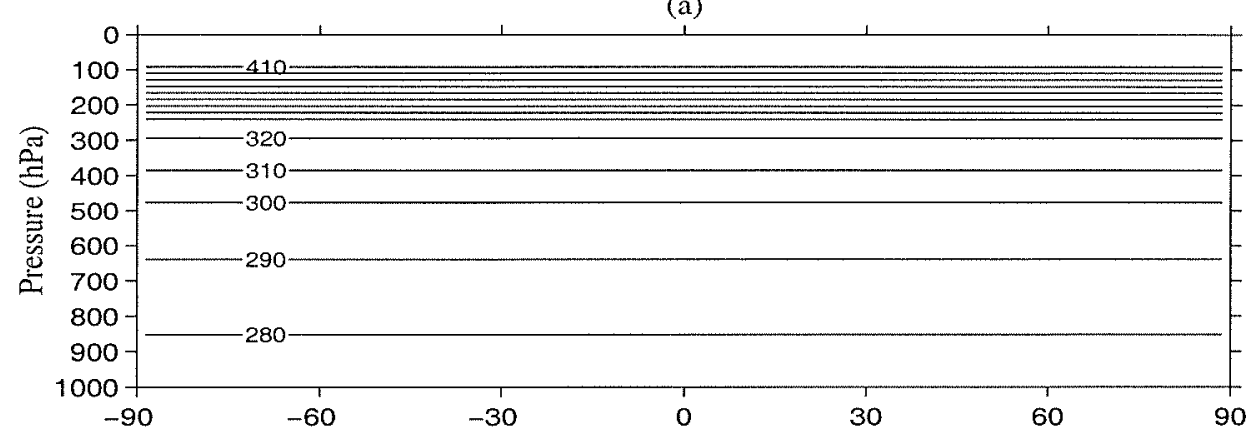

(b)

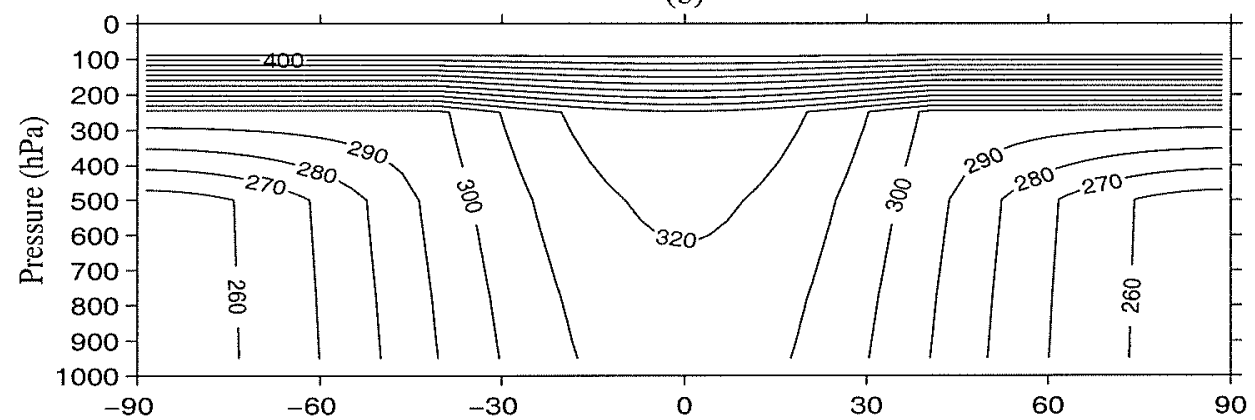

(c)

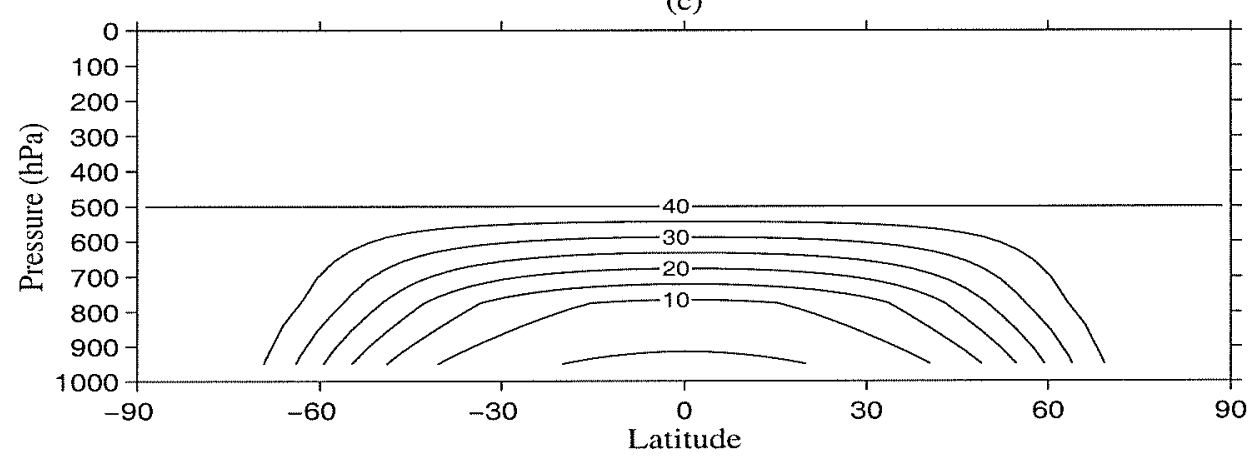

Figure 4.25: Initial meridional cross-sections for the troposphere experiments; (a) potential temperature; (b) $\bar{\theta}_{e q}$, the relaxation potential temperature; (c) $\tau$, the relaxation timescale in days.

radiative-convective equilibrium profile, $\bar{\theta}_{e q}$, on a spatially dependent timescale $\tau$, (figures 4.25.b-c). Results are presented at equilibrium, after 1000 days of integration. 'The zonal momentum constraint is applied independently over each hemisphere to ensure that the eddy transfers in one hemisphere are independent of the PV gradients in the other hemisphere and a $\beta$-plane geometry is used.

The zonal-average model utilized here is ideal for coupled atmosphere-ocean experiments because it correctly captures the transfer characteristics of heat and momentum 
by the atmospheric baroclinic eddies. Such experiments are providing dynamical insights into the role of each fluid in global climate. However, such experiments are computationally expensive if the eddies of each fluid are resolved in the calculations. Our atmospheric experiments are all symmetrically forced about the equator. However in the real atmosphere there is an asymmetry between each hemisphere in the magnitude of the mid-latitude westerly jets and their seasonal variation. The southern hemisphere has a greater fraction of the surface covered by oceans and also has the only oceanic flow not closed off by meridional boundaries: the Antarctic circumpolar current (ACC). Thus it is important that the closure scheme takes into account the local properties for representing the transfer of quantities; for example the southern hemisphere eddies should not be influenced by the PV gradients in the northern hemisphere or vice versa. Our scheme accounts for this by evaluating the $K$ 's in equation 3.10 for each hemisphere individually based on the PV distribution for that hemisphere.

The results presented here were integrated for 1000 days at which point the fields are in a steady state. The initial state is a horizontally stratified atmosphere as shown in figure 4.25.a which is then relaxed to the prescribed radiative-convective equilibrium profile, $\bar{\theta}_{e q}$, on a spatially dependent timescale $\tau$ (figures 4.25 .b-c).

\section{No eddy-forcing}

The importance of the eddy-forcing of the atmospheric general circulation can be most readily seen by suppressing the transfer of momentum and potential temperature by the eddies (ie. setting $\overline{v^{\prime} q^{\prime}}=0$ ) and inspecting the large-scale flow which occurs in their

absence. The resulting flow is axisymmetric consistent with the imposed radiative forcing and the subsynoptic mixing present in the absence of the large-scale eddies.

The potential temperature, zonal velocity, and meridional circulation profiles are shown in Fig.4.26. The potential temperature has relaxed to the prescribed profile resulting in a zonal velocity consisting of two westerly jets with maxima aloft at $25^{\circ}$ latitude. Zonal velocities at $950 \mathrm{mb}$ (Fig.4.26.d) vary between easterlies of $-0.6 \mathrm{~ms}^{-1}$ and west- 


\begin{tabular}{|c|c|c|c|c|}
\hline Parameter & units & No eddy-forcing & Eddy-forcing & Limiting case \\
\hline Bottom drag & $m^{-2}$ & $2.1 \times 10^{-3}$ & $2.1 \times 10^{-3}$ & $2.1 \times 10^{-3}$ \\
\hline$y$-domain & - lat. & -90 to 90 & -90 to 90 & -90 to 90 \\
\hline Height & $\mathrm{hPa}$ & 75 to 950 & 75 to 950 & 75 to 950 \\
\hline Horizontal grid size & - lat. & 2.8125 & 2.8125 & 2.8125 \\
\hline Vertical grid size & $\mathrm{hPa}$ & $100-300$ & $100-300$ & $100-300$ \\
\hline Vertical levels & & 5 & 5 & 5 \\
\hline Horizontal diffusivity & $m^{2} s^{-1}$ & 0 & 0 & 0 \\
\hline Biharmonic diffusivity & $m^{4} s^{-1}$ & $2 \times 10^{15}$ & $2 \times 10^{15}$ & $2 \times 10^{15}$ \\
\hline Vertical diffusivity & $m^{2} s^{-1}$ & 0 & 0 & 0 \\
\hline Horizontal viscosity & $m^{2} s^{-1}$ & 0 & 0 & 0 \\
\hline Biharmonic viscosity & $m^{4} s^{-1}$ & $2 \times 10^{15}$ & $2 \times 10^{15}$ & $2 \times 10^{15}$ \\
\hline Vertical viscosity & $m^{2} s^{-1}$ & 0 & 0 & 0 \\
\hline$K_{p u}$ & $m^{2} s^{-1}$ & 0 & $1 \times 10^{6}$ & $3.4 \times 10^{5}$ \\
\hline $\mathrm{T}(\mathrm{t})$ & & - & 10 day ramp & 10 day ramp \\
\hline$Y(y)$ & & - & $\sin (2 \times \operatorname{lat})$ & $\sin (2 \times l a t)$ \\
\hline
\end{tabular}

Table 4.3: Parameters for the parameterized tropospheric eddy experiments. 
(a)
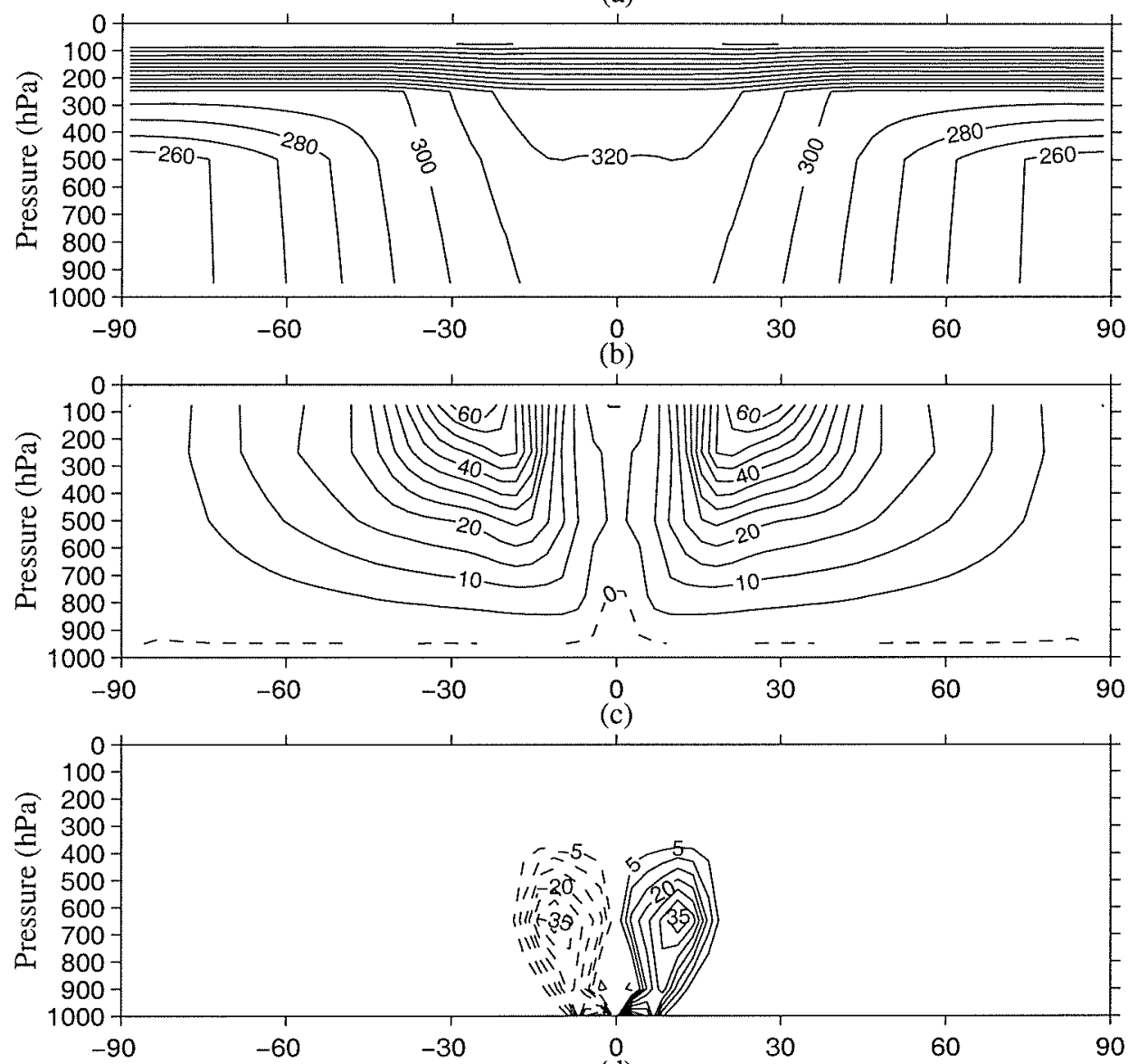

(d)

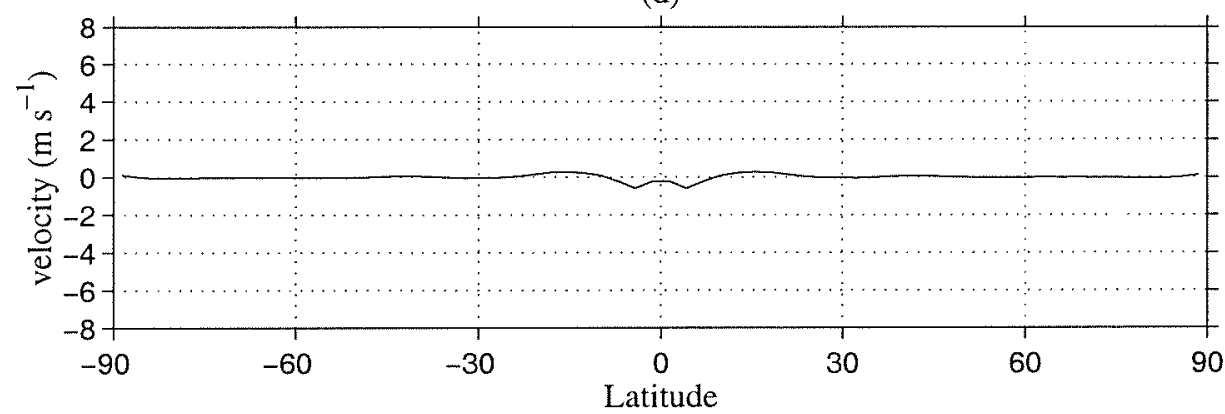

Figure 4.26: The meridional cross-sections after 1000 days for the experiment with no eddy-forcing: (a) potential temperature; (b) zonal velocity; (c) residual mean overturning streamfunction; (d) $950 \mathrm{mb}$ winds.

erlies of $0.35 \mathrm{~ms}^{-1}$. Since the eddy flux of quasigeostrophic potential vorticity is zero, the residual mean circulation is exactly equal to the Eulerian mean circulation and this 
zonally averaged meridional circulation appears as Hadley cells in each hemisphere (see Fig.4.26.c). Low level winds are easterly at the equator where the warm air rises and are westerly at low levels where the air in the Hadley cell subsides; this provides a zero torque on the atmosphere necessary for the steady state. In the extratropics because of angular momentum constraints there is no meridional motion. The equilibrium zonal flow and potential temperature fields is set by the nature of the restoring terms (Held and Hou (1980)).

\section{Eddy-forcing}

The approach of chapters 2 and 3 is now employed. The reference value of the transfer coefficient is prescribed to be $\kappa_{r e f}=1 \times 10^{6} \mathrm{~m}^{2} \mathrm{~s}^{-1}$ with the model evaluating $K$ at each latitude and pressure. Again, if the potential vorticity distribution does not satisfy the necessary conditions for instability then the $K$ 's are set to zero and the eddies do not force the mean flow. Once the midlatitude jets can support baroclinic instability the $K$ 's become non-zero and are linearly ramped up over a 30 day period. This crudely simulates the growth of the eddies whose flux will grow as they reach finite amplitude. At steady state the flow in both hemispheres is characterized by westerly jets with maxima of $35 \mathrm{~ms}^{-1}$ at about $38^{\circ}$ latitude near the tropopause. Zonal velocities at $950 \mathrm{mb}$ display equatorial easterlies of $-5.1 \mathrm{~ms}^{-1}$, midlatitude westerlies of $6.0 \mathrm{~ms}^{-1}$, and weak polar easterlies. The residual mean streamfunction consists of a single overturning cell in each hemisphere extending further poleward than the Hadley cells in the previous experiment. However, we stress that these cells are not the Hadley cells which appear in the Eulerian mean formalism; they are the cells of the transformed Eulerian mean. The potential temperature relaxation leads to diabatic heating in the tropics where fluid parcels rise and cooling at high latitudes where they subside. Thus the residual mean circulation approximately represents the mean motion of the air parcels. It is poleward aloft with return flow at low levels. In our channel ocean experiments there were no sources or sinks of temperature, the motion was adiabatic and hence the residual mean overturning 
(a)

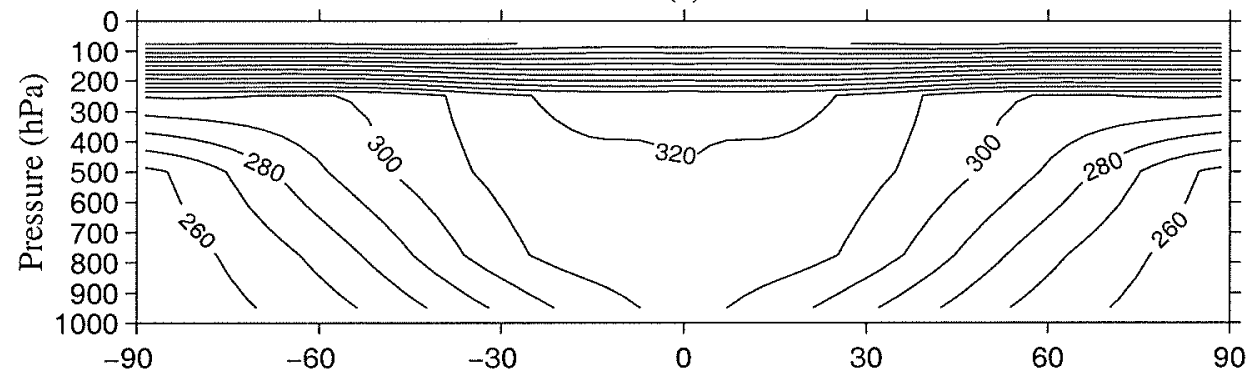

(b)

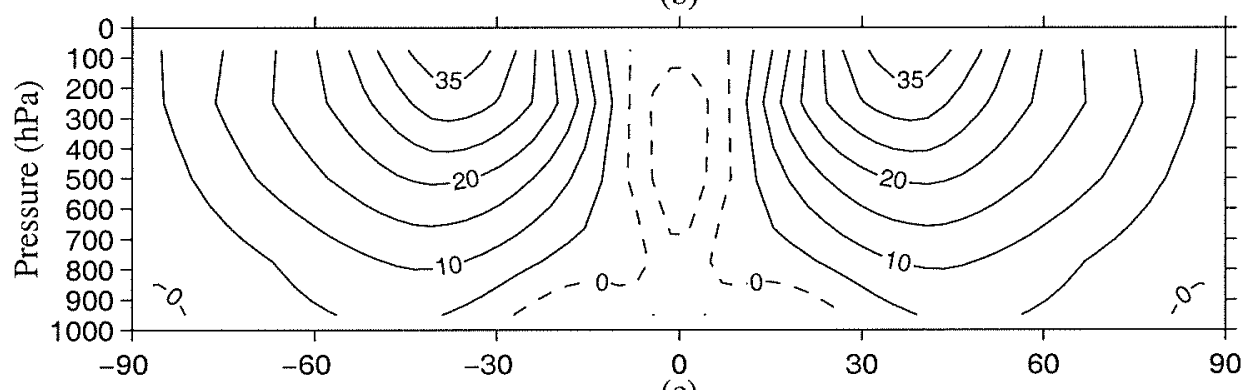

(c)
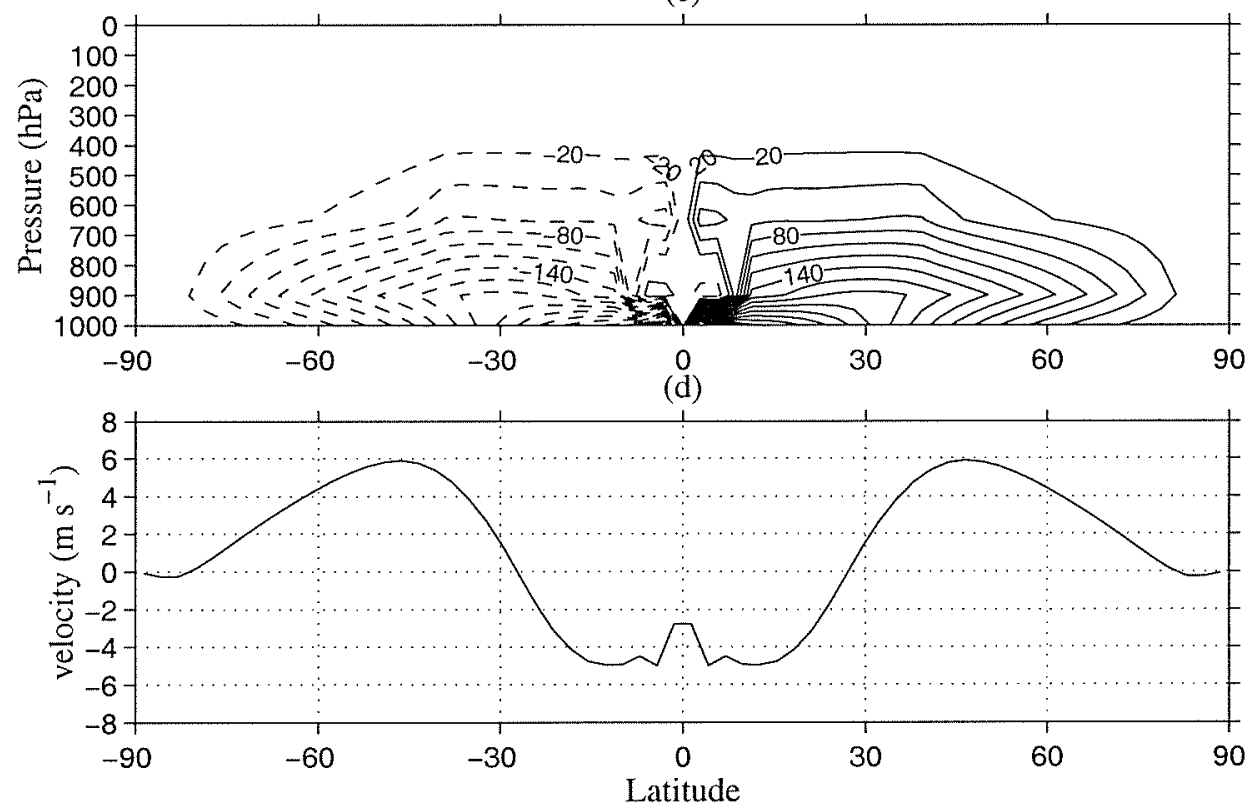

Figure 4.27: The meridional cross-sections after 1000 days for the experiment with eddyforcing: (a) potential temperature; (b) zonal velocity; (c) residual mean overturning streamfunction; (d) $950 \mathrm{mb}$ winds.

motion vanished. Here the meridional motion does not vanish due to the diabatic forcing. The eddy PV flux in the meridional plane is plotted in figure 4.28.a. At the surface 

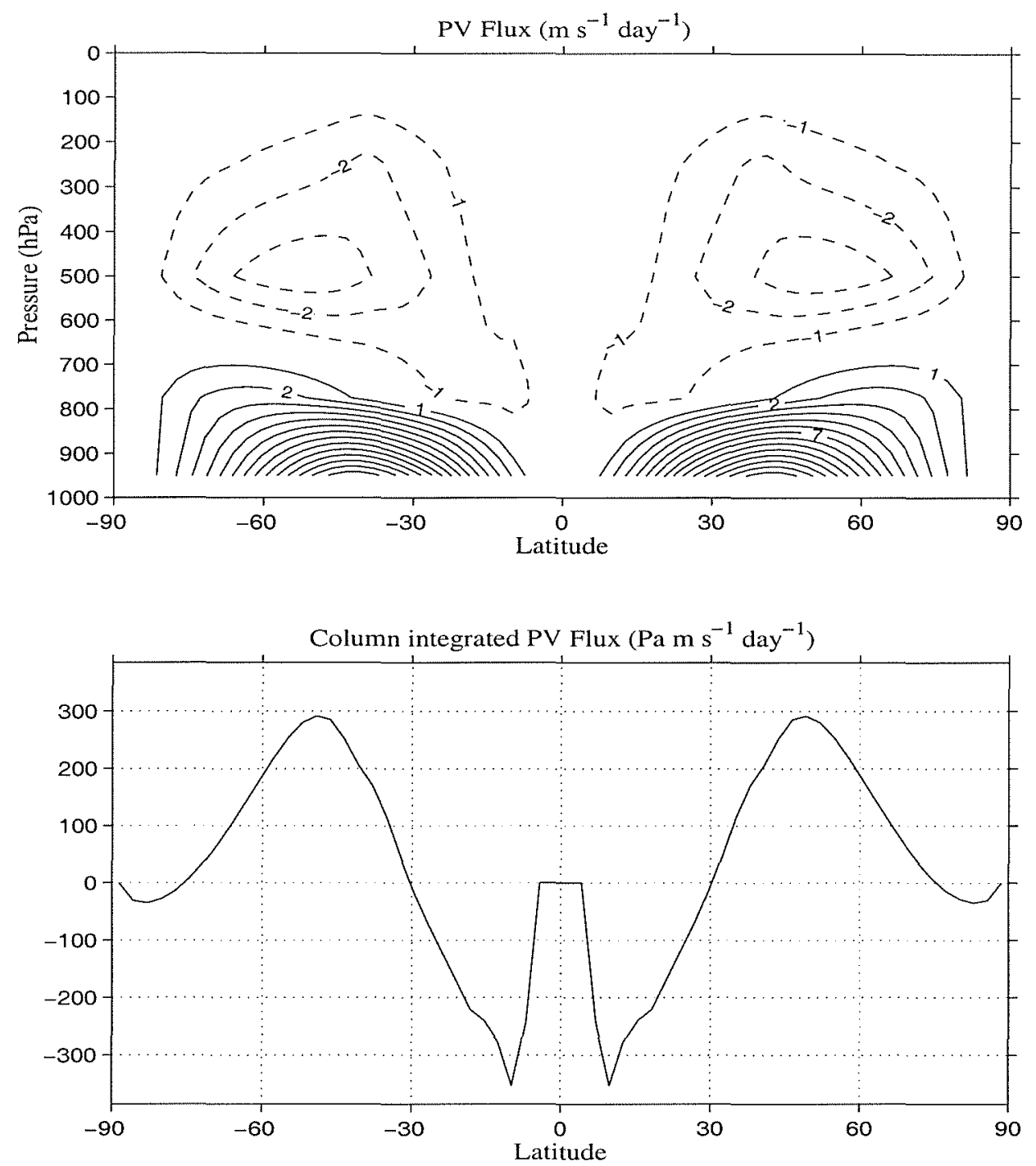

Figure 4.28: (a). The Eliassen-Palm flux divergence; (b) The column integrated EliassenPalm flux divergence. The eddies exert a westerly force at mid-latitudes and easterly forces in the tropics and toward the poles.

there is a potential vorticity sheet due to the potential temperature perturbations along the boundary. There is a northward potential vorticity flux corresponding to EliassenPalm flux divergence. The compensating convergence occurs at most heights in the extratropical troposphere. This map of eddy PV flux agrees well both in form and magnitude with maps diagnosed from atmospheric analyzed fields - see, for example, 
Schubert et al (1990) using ECMWF fields. Integrating the zonal momentum equation over each column gives a three-way balance between the eddy-forcing term, the meridional advection of zonal flow by the residual mean $\left(\bar{v}^{\star} \bar{u}_{y}\right)$, and the bottom drag. The columnintegrated Eliassen-palm flux divergence (figure 4.28.b) is positive in midlatitudes and negative at the equator and poles. Thus there is a column-integrated $E_{y}$ which points from midlatitudes to the equator south of the westerlies and from midlatitudes to the pole to the north; the column-integrated momentum flux is directed toward midlatitudes from the flanks of the westerly jets. The result is lateral momentum transfer which shifts the jet center northwards from $25^{\circ}$ to $38^{\circ}$ latitude and gives midlatitude surface westerlies. The meridional profiles obtained in the parameterized model compare well to zonal-mean cross sections of the zonal wind component for observed annual conditions shown in figure 7.15a in Peixoto and Oort (1992). The only striking difference between the observed and modeled jets is the lack of distinct cores at height in the model. This is likely to be due to the low vertical resolution of the model at these levels.

\section{Eddy-forcing: zero Reynolds stresses}

We now neglect the relative vorticity fluxes in equation 2.15 as we did for the spin-down of the baroclinic zone flow to reduce our scheme to that of Gent and McWilliams. After 1000 days the equilibrium fields are characterized by westerly jets in each hemisphere with maxima at $25^{\circ}$ latitude with values of $45 \mathrm{~ms}^{-1}$ (figure 4.29.b). The meridional profile potential temperature (figure 4.29.a) is similar to that of the no eddy-forcing case. The residual overturning circulation extends toward the poles with a structure similar to that of the eddy-forced experiment with maximum value that is $60 \%$ less. The meridional cross-section of the Eliassen-Palm flux divergence in the equilibrated state is plotted in figure 4.30.a. It shows that like the eddy-forced run there is Eliassen-Palm flux divergence at the lower boundary with compensating convergence at mid-heights in the troposphere. However the column-integrated Eliassen-palm flux divergence (figure 4.30.b) is zero because relative vorticity fluxes have been ignored. There is no lateral 
(a)
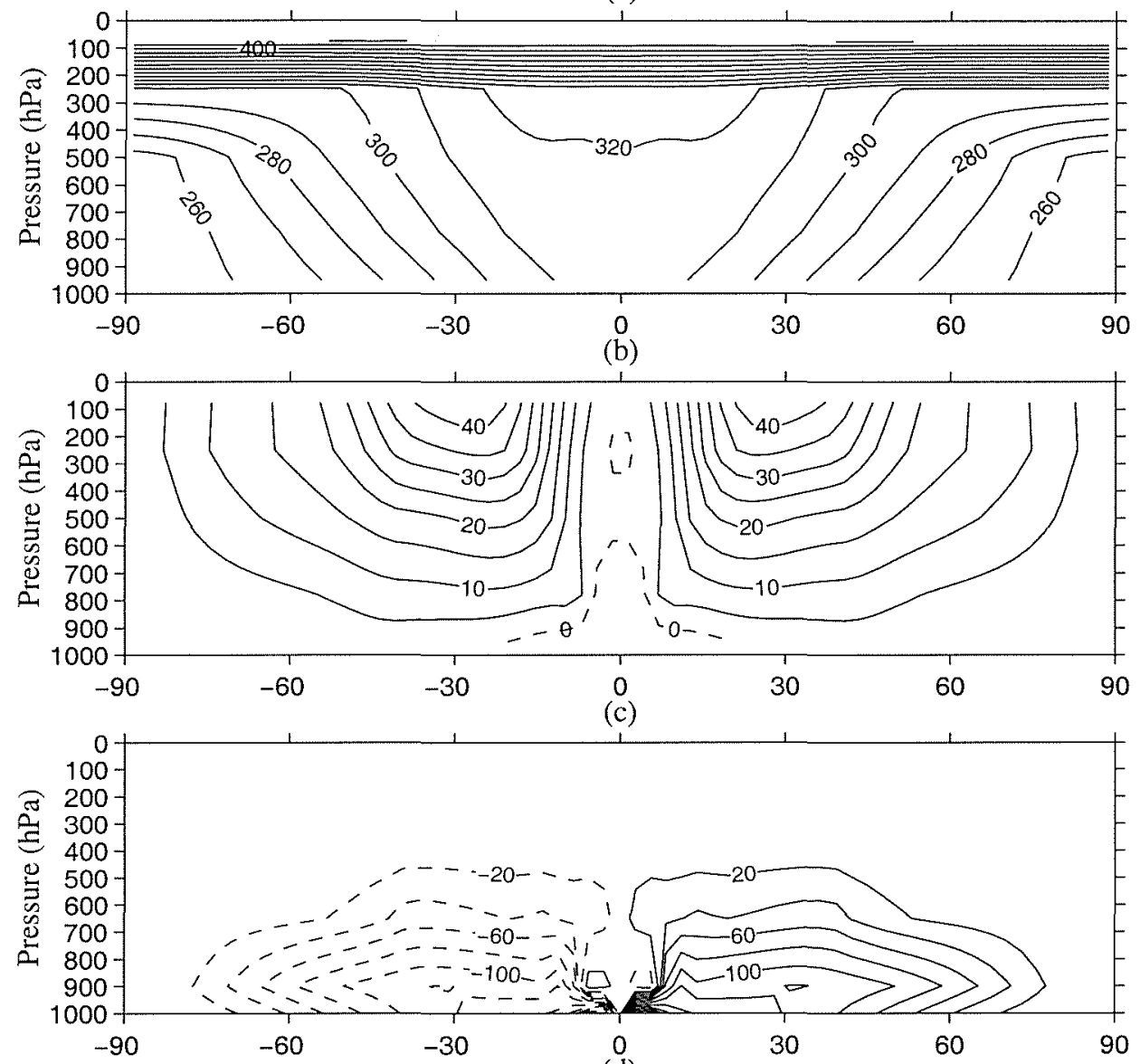

(d)

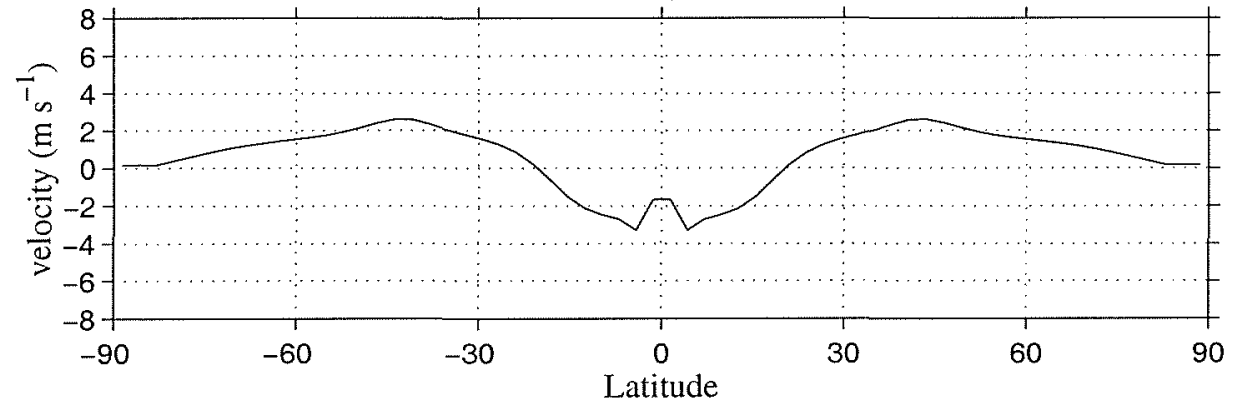

Figure 4.29: The meridional profiles after 1000 days for the experiment with the limiting case of the eddy-forcing: (a) potential temperature; (b) zonal velocity; (c) residual mean overturning streamfunction; (d) $950 \mathrm{mb}$ winds.

momentum flux $\left(E_{y}=0\right)$. There is only vertical transfer of momentum, due to the lateral eddy buoyancy fluxes, which reduces the shear of the westerly jets and increases 

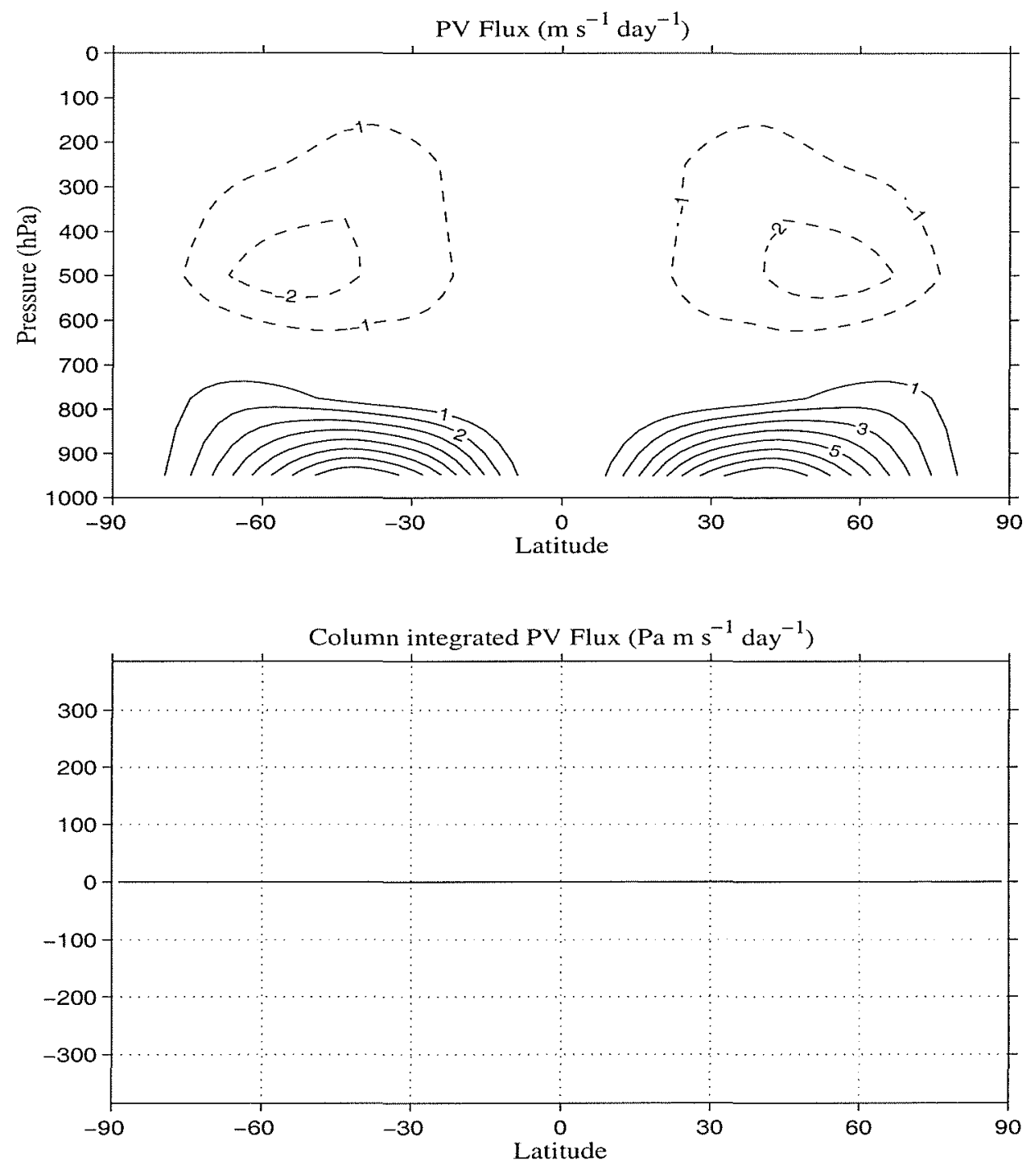

Figure 4.30: The meridional cross-sections of; (a) the Eliassen-Palm flux divergence; (b) the column integrated Eliassen-Palm flux divergence. The column-integrated divergence is zero because relative vorticity fluxes have been ignored. There is no lateral momentum flux $\left(E_{y}=0\right)$ and so there is only vertical transfer of momentum, due to the lateral eddy buoyancy fluxes. 
the low-level winds (see figure 4.30.d). However, the eddy-forcing of the mean-flow is unable to change the position the jet cores and sharpen the midlatitude westerlies.

It is well known that the tropospheric circulation cannot be modeled purely in terms of zonally symmetric processes; eddy buoyancy and momentum fluxes are crucial to the observed meridional structure and must be appropriately represented in order to achieve a realistic circulation. The three atmospheric experiments presented here clearly show that a realistic picture of the vertical and meridional distributions of mean zonal flow can only be attained when the full transfer characteristics of the eddies are represented.

\subsection{Summary}

In this chapter, the 'transformed Eulerian mean' approach for zonal mean flows, introduced in chapter 2, was implemented in a hydrostatic primitive equation model.

The eddy PV flux was assumed to be transferred down its mean gradient with a transfer coefficient $K$ (equation 3.5). The form of the $K$ 's are chosen to ensure that an integral constraint on the eddy flux is satisfied, so that the eddies act only to redistribute momentum in the flow. Downgradient PV flux does not, however, necessarily imply that momentum is diffused downgradient (e.g. $\left.\overline{u^{\prime} v^{\prime}}=-k \bar{u}_{y}\right)$. Indeed the approach can capture the sharpening of jets due to eddy-mean flow interaction on a $\beta$-plane.

The first experiment was performed for stress-driven flow in a $\beta$-plane channel. The mean fields and eddy transfer characteristics of the eddy-resolving flow were compared to those of the same stress-driven flow in a parameterized model. The comparison shows that the transformed Eulerian mean approach offers advantages over existing parameterization schemes. The zonal mean fields of the parameterized model closely matched those of the eddy resolving calculation in the equilibrated state.

The second experiment concerned the spin-down of a baroclinic zone on a $\beta$-plane. It highlighted a further advantage of representing eddies through a PV flux. Because the approach is based on gradients of PV, necessary conditions for instability are built into the 
parameterization scheme. This leads to no PV flux by the eddies when necessary conditions are not met and stabilization of jets by the planetary PV gradient ( $\beta$-stabilization). This feature is absent from the Gent and McWilliams approach, resulting in vanishing of jet currents that are not maintained by diabatic processes.

The implementation of the scheme in an atmospheric model yielded a realistic picture of zonal mean flow when compared to an implementation of the GM approach. Eddy processes are an order one process in the atmosphere, and as the experiments show, the correct zonal mean physics can only be attained when the full transfer characteristics of the quasigeostrophic eddies are represented.

This chapter focused on problems that displayed a marked symmetry in the direction of the mean flow because it offers the simplest setting in which to explore. With the zonal mean flows, the zonal symmetry displayed by the eddy statistics are implicit by design, because there is no variation in the mean in the direction of the flow. However, in the absence of a zonal-symmetric mean flow, nonlocal contributions of eddy enstrophy may result in eddy fluxes of PV that are directed up the mean PV gradient. We address this fact in the light of the parameterization scheme, in chapter 5. 


\section{Chapter 5}

\section{Time Mean Flows}

\section{$5.1 \quad$ Introduction}

In the previous chapter we studied the parameterization of quasigeostrophic eddies in zonal mean flows in the ocean and atmosphere. The TEM approach and the flux-gradient transfer closure was successful in producing mean fields and eddy statistics that compared well to those from eddy resolving calculations. In addition to capturing the transport characteristics, the TEM framework led to an increased understanding of eddy propagation and eddy forcing of the zonal-mean flow.

However, there is a large body of flows in the ocean for which a zonal-mean perspective is not appropriate. The ocean, unlike the atmosphere, is not zonally unbounded. With the exception of the Southern Ocean, all oceans are laterally bounded by continental land masses which results in a breaking of the zonal symmetry. The closed ocean basins can support zonal pressure gradients which lead to geostrophic meridional velocities and a non-conserved along-flow component of the velocity field. This meridional velocity is in Sverdrup balance (Sverdrup (1947)), in which the vertically integrated meridional velocity is proportional to the curl of the wind stress. This relation is the cornerstone of winddriven ocean circulation theory, explaining the sense of rotation and the mass transport of major ocean gyres. Thus, the first-order oceanic circulation can be understood without 
the need to solicit the transfer by the eddy field. This is in contrast, as section 4.4 showed, to the large-scale circulation of the atmosphere in which the eddy transfer is crucial to the observed meridional structure of the zonal mean flow.

The existence of the interior Sverdrup solution requires, for mass balance of the whole ocean basin, a compensating meridional transport opposite to that in the ocean interior. This occurs in intense western boundary currents where the dynamical balance differs from that in the interior Sverdrup flow. These western boundary jets are prone to instability which generates eddies locally in the jet region and radiates energy into the Sverdrup interior, see Pedlosky (1977). In the absence of the zonally symmetric mean flow, regions of eddy generation are more localized and advection of eddy PV variance by the mean flow may give rise to a change in the nature of the eddy statistics from that of zonal mean flows. Thus it is more suitable for modeling general ocean flows, to apply a time mean rather than a spatial mean.

In this chapter, the TEM equations set 2.60.a-e, along with the flux-gradient closure (equation 3.5) for the eddy PV flux, is examined and explored in the context of an eddy resolving three-dimensional flow. In section 5.2, a double-gyre ocean experiment is presented in the light of the theory laid out in chapter 2. Section 5.3 details how one could devise a prognostic model of time mean ocean/atmosphere flows in which the eddy closure is through a PV flux term.

\subsection{Flow in a double-gyre ocean}

In this section the methodology outlined in chapters 2 and 3 is explored in a double-gyre, eddy-resolving numerical simulation. The eddy statistics of interest are computed and interpreted from the point of view of the time mean TEM theory. The utility of such an approach is that it can be used to examine and build our intuition about the nature of the eddy transfer in a flows where it is not appropriate to average spatially. 


\subsubsection{Model setup}

\section{Configuration}

The numerical model solves for the wind-driven ocean flow in a closed rectangular domain. The longitudinal and latitudinal extents are $2400 \mathrm{~km}$ and $2000 \mathrm{~km}$ respectively, with a flat bottom at depth $1000 \mathrm{~m}$ (see figure 5.1). As with the channel experiments in the

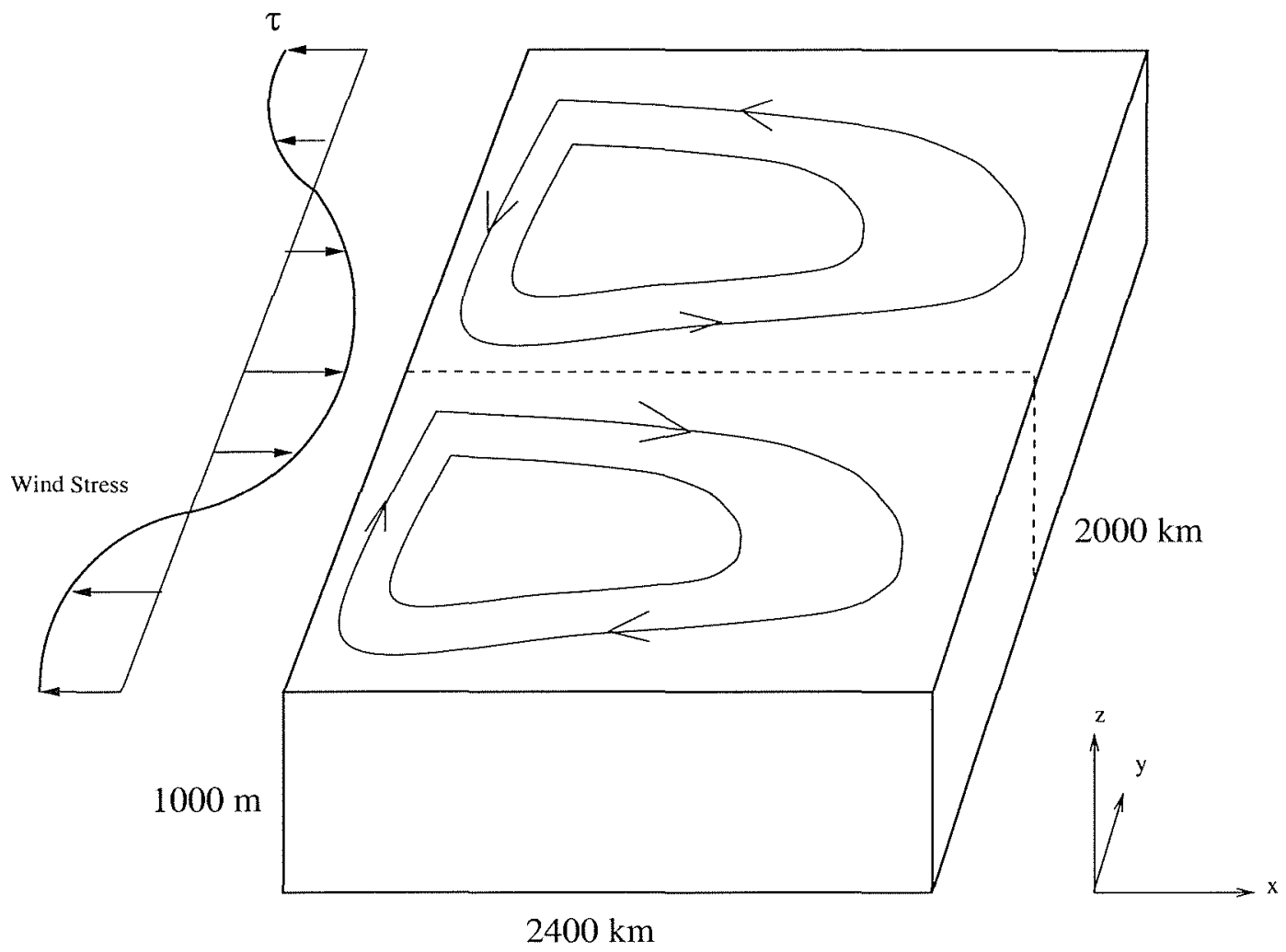

Figure 5.1: A schematic diagram of the double-gyre model domain. A sinusoidal wind stress drives subtropical and subpolar gyres to the south and north of the zero wind stress curl line.

chapter 4, the initial stratification is constant. The vertical grid spacing was $100 \mathrm{~m}$ in the vertical. Friction is present through a bottom drag in the lower layer, Laplacian and biharmonic viscosity is used. Biharmonic diffusivity is used to suppress numerical noise 
on the grid-scale. To exclude a thermally driven circulation, no thermodynamic forcing $(G=0)$ is used in the experiment. Static instability is released by convective adjustment. The numerical experiment is summarized in Table 5.1. The equation of state is a linear function of temperature only, with salinity effects neglected. The applied wind-stress is applied in the upper level of the model and has the form:

$$
\tau=-\tau_{o} \cos \left(\frac{\pi y}{L_{y}}\right)
$$

where $L_{y}$ is the meridional extent of the basin. This produces a double-gyre flow pattern with a northern cyclonic subpolar gyre, and a southern subtropical gyre of anticyclonic rotation.

\section{Length scales and jet penetration}

Extensive studies of homogeneous ocean models (see Pedlosky (1996) for a comprehensive review) identify several length scales of the western boundary current that are of interest in the baroclinic ocean flow at hand. The dynamics of the boundary current can be either frictional or inertial. If the dynamics are inertial (Fofonoff (1954), Charney (1955), Morgan (1956)), the boundary layer scales as:

$$
\delta_{I}=\left(\frac{U}{\beta}\right)^{1 / 2}
$$

where $U$ is the scale of the zonal velocity. Using Sverdrup balance to give an estimate for $U$, equation 5.2 can be rewritten as;

$$
\delta_{I}=\left(\frac{\pi \tau_{o}}{\rho_{o} \beta^{2} H L_{y}}\right)^{1 / 2}
$$

where $\tau_{o}$ is the maximum of the wind-stress, $\beta$ is the variation of the Coriolis parameter, $H$ is the depth of the wind-driven flow, and $L_{y}$ is the latitudinal extent.

For linear or very weakly non-linear flow, the Sverdrup solution is closed by frictional dynamics. Modeling the oceanic potential vorticity dissipation as a large-scale diffusive process, Munk (1950) showed that the vorticity balance in the western boundary consists 


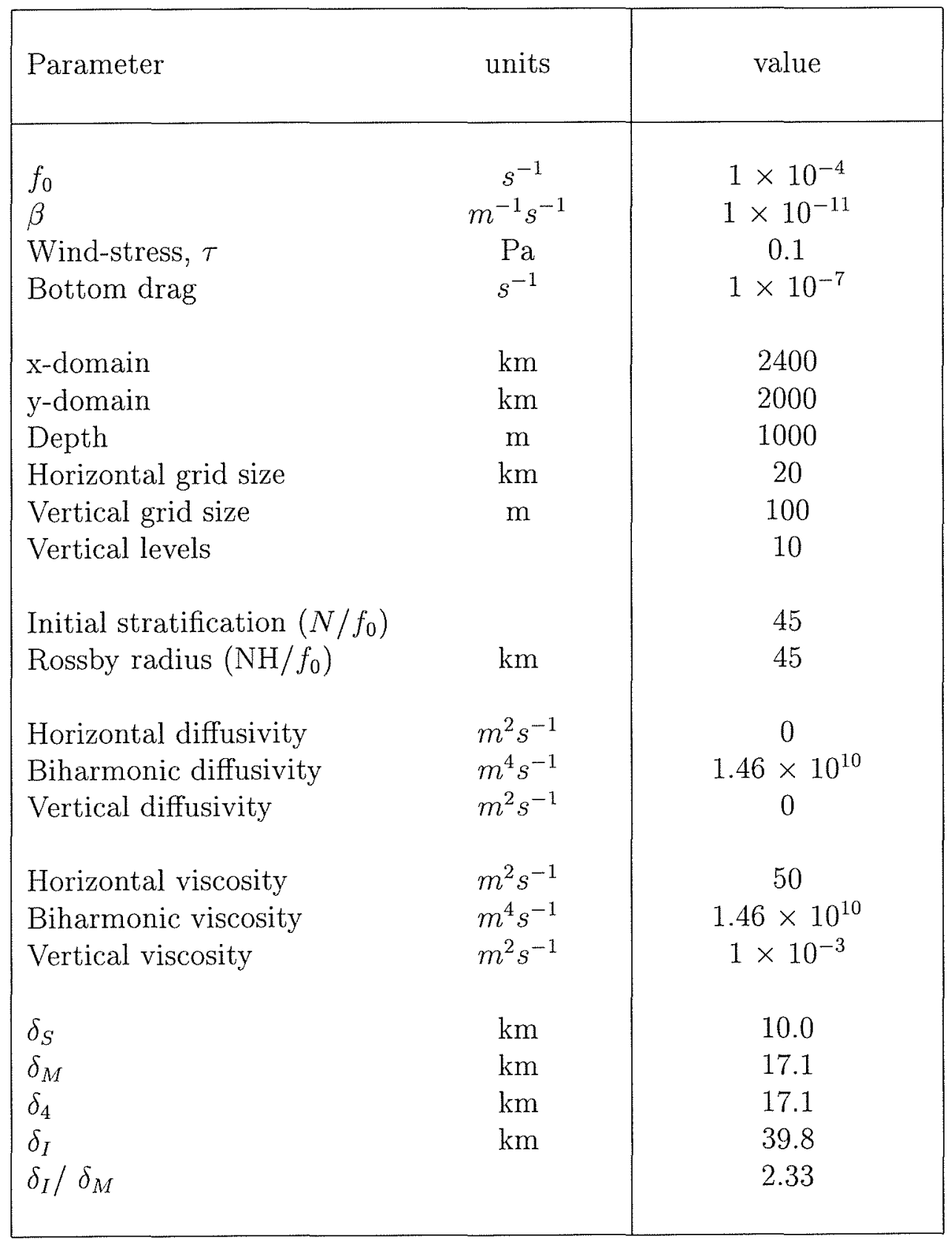

Table 5.1: Parameters for the eddy-resolving double-gyre experiment. 
of Fickian diffusion of vorticity balancing the advection of planetary vorticity. Under this balance, the zonal scale of the boundary layer is given by

$$
\delta_{M}=\left(\frac{A_{H}}{\beta}\right)^{1 / 3},
$$

where $A_{H}$ is the Fickian "eddy viscosity". (Note that Munk represented the momentum flux by the eddies as a diffusive process.) If the dissipation mechanism is represented by biharmonic viscosity then the boundary layer scale is

$$
\delta_{4}=\left(\frac{A_{4}}{\beta}\right)^{1 / 5}
$$

where $A_{4}$ is the biharmonic "eddy viscosity". When bottom friction is present vorticity can be dissipated without the need for lateral diffusion (Stommel (1948)) and the boundary layer then scales as

$$
\delta_{S}=\frac{\epsilon}{\beta}
$$

where $\epsilon$ is the bottom drag coefficient. This is Stommel's (1948) solution for closing the Sverdrup interior balance.

The relative size of $\delta_{I}$ to $\delta_{M}, \delta_{4}$, and $\delta_{S}$ is the measure of the how inertial the flow is in the homogeneous ocean models (see, for example, Veronis (1966), Holland and Lin (1975) Cessi et al. (1990)). For baroclinic flows, instabilities give rise to quasigeostrophic eddies and their associated Reynolds stresses become the prevailing dissipative mechanism. Thus the relevance of the frictional boundary scales for a stratified ocean model is somewhat unclear. However numerical simulations in two-layer quasigeostrophic flows (Holland (1978), Holland and Rhines (1980)) show that when the inertial boundary scale is larger than that of the frictional scale the flow displays turbulent characteristics and a vigorous eddy field.

For single gyre flows, the western boundary must dissipate the vorticity imparted by the wind stress to achieve a steady state. Neglect of lateral friction (High Reynolds number) leads to a circulation in which the interior velocities must be large in order for 
bottom friction to achieve the required vorticity dissipation. The resultant flow is the Fofonoff (1954) free basin mode.

In contrast, for a double-gyre model in which the forcing is symmetric about the midlatitude, the net vorticity input into the gyre is zero. However, as Marshall (1984) shows for a barotropic double gyre the flow does not approach a double Fofonoff mode, even though the flow is highly nonlinear. This is because the interior jet which separates each gyre becomes dynamically unstable and results in lateral transfer of vorticity by eddies that repeatedly form over most of the basin. For these double-gyre flows, the boundary currents merely play the role of closing the mass flux budget.

The matter of the extent of the penetration of the jet and its meandering streamlines is a much studied problem in its own right. For example, Holland and Schmitz (1985), Greatbach (1988), Marshall and Marshall (1992) have all considered the problem of jet penetration scale in numerical ocean models. Marshall and Marshall (1992) show the sensitivity of jet penetration to the choice of boundary conditions, and conclude that free-slip conditions yield PV distributions that are more favorable to deeper penetration into the basin interior. For this reason, the experiment is run with free slip boundary conditions for velocity. As Table 5.1 indicates, the ratio $\delta_{I} / \delta_{M}$ is $2.33(>1)$. Consequently, we expect a jet which penetrates far into the interior, which spawns an energetic eddy field.

\subsubsection{Flow evolution and equilibration}

The time development of the surface temperature and velocity fields is shown in figure 5.2. After 6 months the subtropical and subpolar gyres are separated by an inertial jet that penetrates to $2000 \mathrm{~km}$ ( $83 \%$ of the longitudinal extent) which is marked by the tight temperature contours at $\mathrm{y}=1000 \mathrm{~km}$. Recirculation gyres are present toward the eastward end of the jet. After 1 year, meanders in the jet are visible, with the jet curling tight at its eastern most extent. Eddies have pinched off, and are visible to the east of the jet and in the subtropical gyre. The subsequent snapshots (each presented here in 

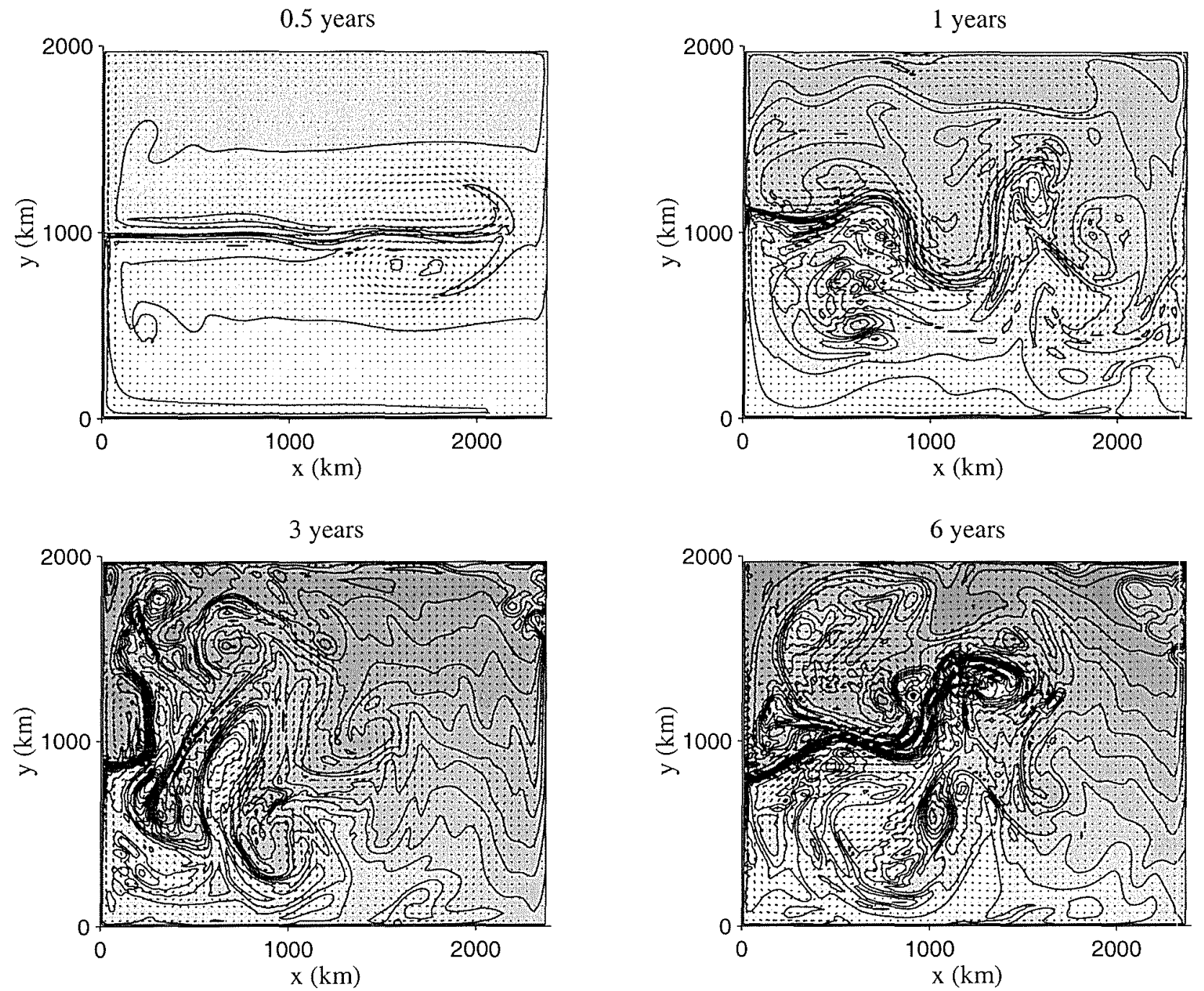

Figure 5.2: Instantaneous surface temperature and velocity fields for the double-gyre experiment at (a) 0.5 years, (b) 1 year, (c) 3 years, (d) 6 years, (e) 10 years, (f) 15 years, (g) 18 years, (h) 25 years. The temperature field is contoured with warmer water denoted by lighter shading. Velocities are denoted by arrows. 

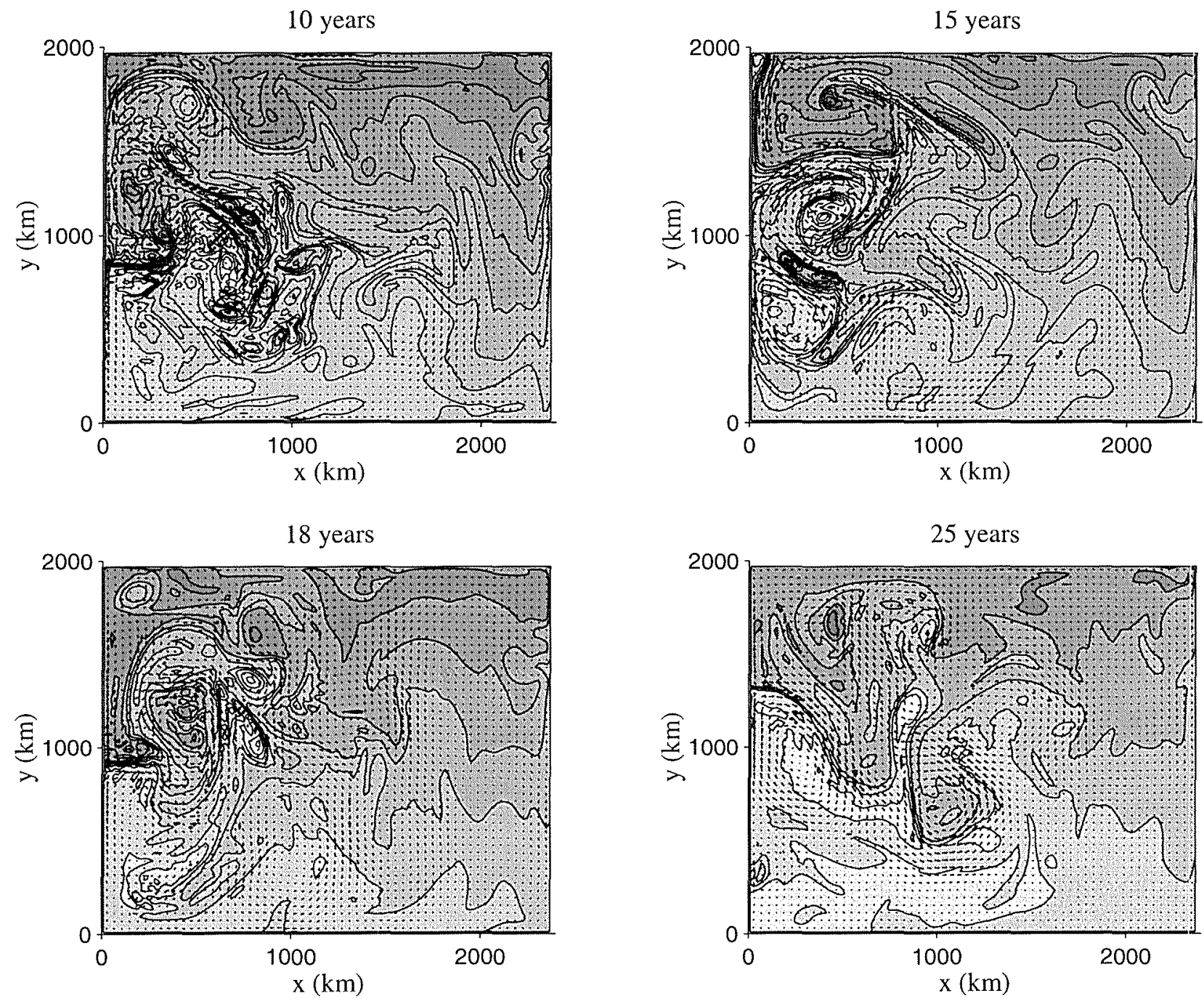

Figure 5.2 (Continued) 
multiples of 1 year) show high variability in the jet separation point from the western boundary (e.g. compare years 1 and 3), jet penetration (c.f. years 6 and 8), and the number of eddies present in the gyres (c.f. years 6 and 25). At some times, the jet is strongly marked (6 years), at others is less pronounced (18 years), occasionally it will split into multiple jets (15 years), and at other times filamentation associated with the cascade of vorticity is evident (25 years). This marked variability is evident in the time

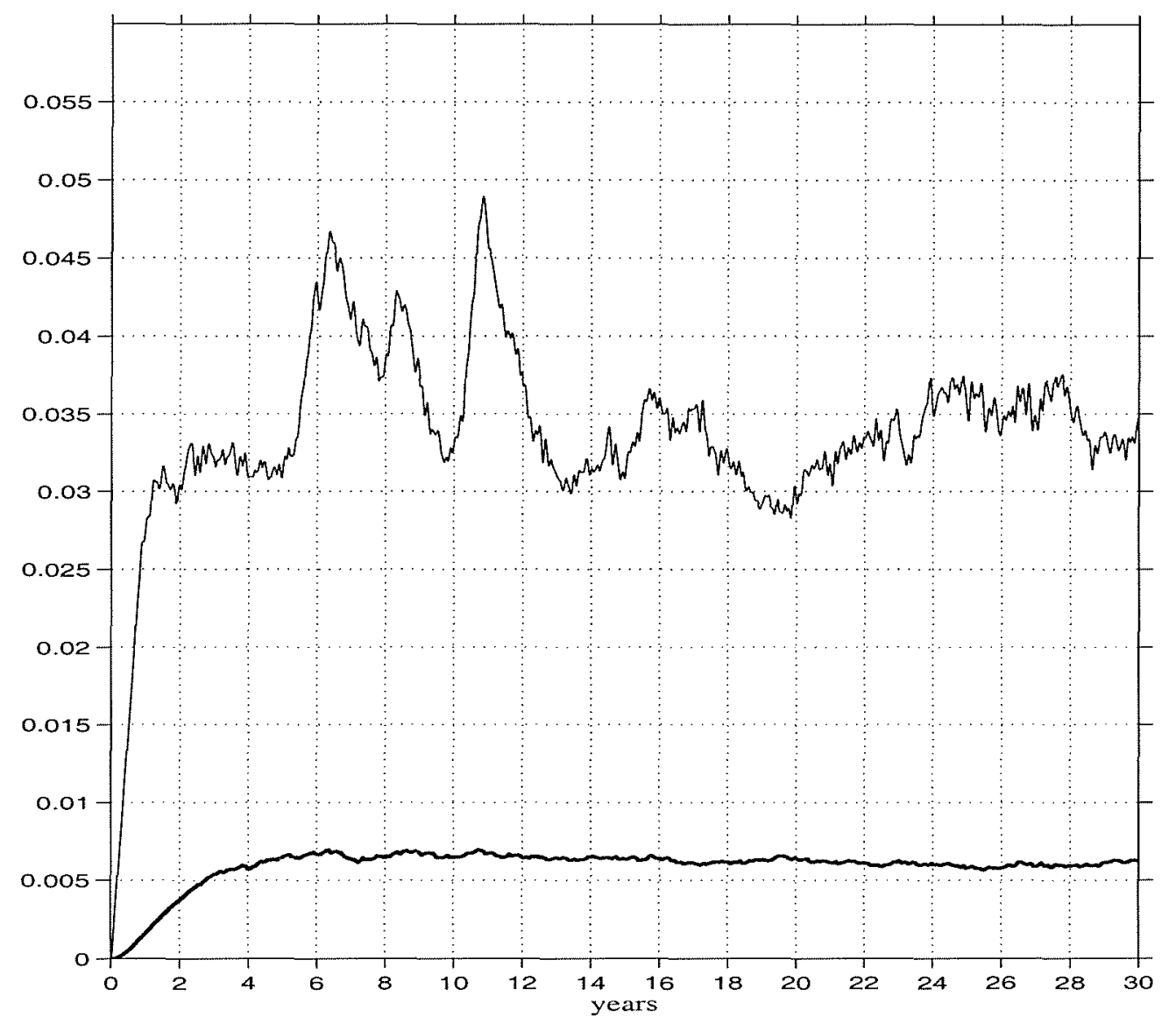

Figure 5.3: The times series of the available potential energy (heavy line) and kinetic energy (thin line) per unit mass per unit volume.

series of average mean kinetic energy presented in figure 5.3. The energy is characterized by variations from short time scales to the inter-annual. This variability and its generation mechanism is an interesting problem in its own right [see, for example Primeau 
(1998) who shows that bifurcation theory is useful in explaining the time mean and low frequency variability of simple double-gyre models] but will not be pursued here. The kinetic energy is a factor of 5 times larger than the available potential energy in the run presented here. This suggests that the primary eddy generation mechanism is barotropic instability resulting from the horizontal shears in the flow. [Another experiment that is not reported in the thesis had the kinetic energy and available potential energy with comparable values, and much more of a mixture of both barotropic and baroclinic eddy generation. The results differ only quantitatively from those that will be presented here. Indeed, the physics of the instability and its generation mechanism appears in equation 3.5 through the structure and size of the transfer coefficients $K$. Because the principal eddy generation mechanism is not baroclinic instability does not invalidate the fluxgradient closure relationship (equation 3.5.)] The calculation is integrated for 30 years.

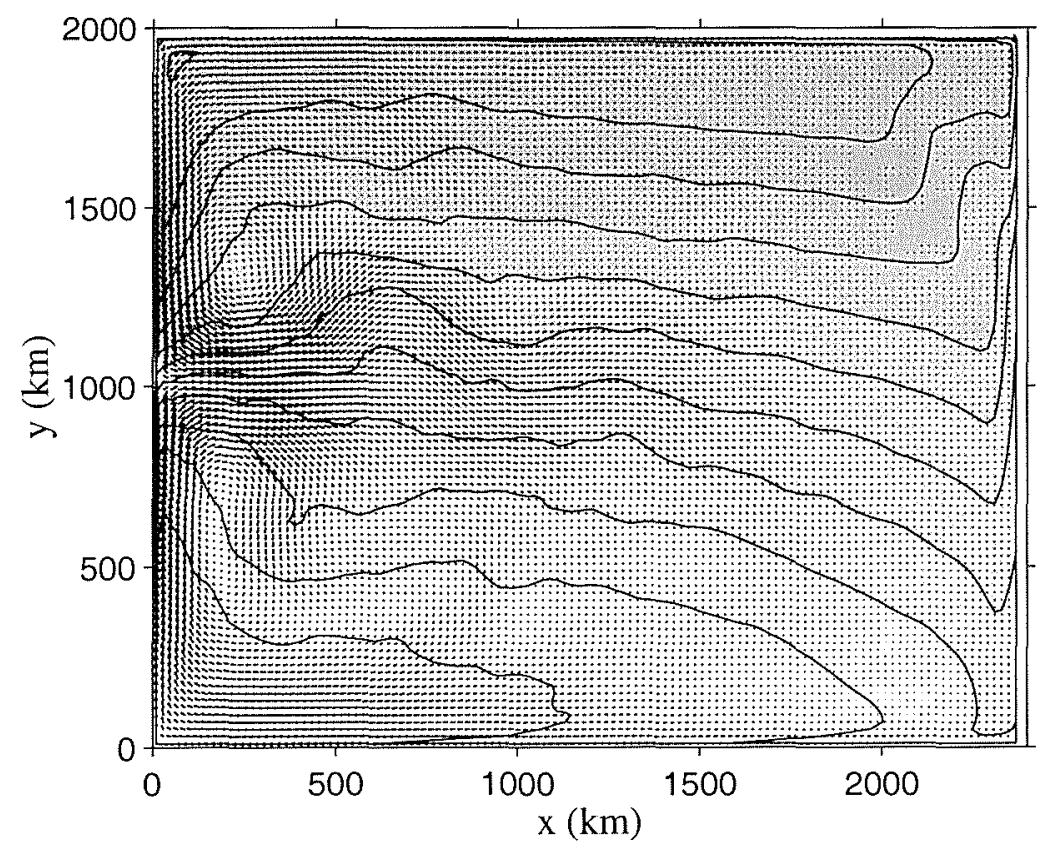

Figure 5.4: Time mean surface velocity and temperature fields

Figure 5.4 presents the time mean temperature and surface velocities, averaged over the 
last 12 years (years $18-30$ ) of the model integration.

The time mean velocities in the western boundary current reach a maxima of 64 $\mathrm{cms}^{-1}$, with typical velocities in the eastward flowing, interior jet of $24 \mathrm{cms}^{-1}$. Velocities in the gyre interior are on the order of $2 \mathrm{cms}^{-1}$, consistent with Sverdrup theory which predicts a value of $1.57 \mathrm{cms}^{-1}$ with the parameters listed in Table 5.1. The maximum surface temperature in the subtropical gyre is $12.5^{\circ} \mathrm{C}$, while the minimum in the subpolar gyre is $12.2^{\circ} \mathrm{C}$. Thus meridional temperature gradients are smaller than observed in the real ocean.

The Eulerian mean barotropic streamfunction is shown in figure 5.5.a. The circulation is comprised of the subtropical and subpolar gyres separated by an eastward flowing jet at midlatitudes with Sverdrup return flow in the interior. The depth-integrated mass transport of each gyre is $40 \mathrm{~Sv}$. Because the forcing is symmetric about the middlelatitude, the flow field should be antisymmetric in each basin. However, because of the average is taken over a finite time (and therefore a finite number of eddies), the $\bar{\psi}$ field has minor asymmetries. Figure 5.5.b depicts the PV distribution in the upper level and hence contains the contribution from the PV sheet. One feature is evident in figures 5.4 and 5.5.b that is not given by Sverdrup or western boundary current theories. It appears in the northeastern corner of the model domain and I shall refer to it as the "northeastern feature". It arises from the C-grid discretization of the model. The wind stress imparted on the fluid is independent of longitude. Sverdrup balance predicts that the shear of the vertical velocity (and hence the $w$ itself) is therefore also independent of $\mathrm{x}$. However, at the eastern boundary, the C-grid discretization gives a $w$ that differs from that in the basin interior. As a result, the advective balances for temperature are different next to the eastern wall than in the interior, which manifest themselves as the "northeastern feature". As we shall see, this unfortunate structure, which is a purely numerically generated feature, will not impact the diagnostics and conclusions of the eddy transfer statistics.

In figure 5.6, the PV distribution for each level in the model is plotted. The effect 
of the temperature gradients at the surface manifest themselves in the PV sheet in the upper level. The mean PV gradients at this level are typically a factor of 10 larger than the planetary PV gradient, $\beta$. In the eddy intense region this factor is 30 . At subsequent levels the mean PV gradients are much weaker than in the sheet and are typically an order of magnitude smaller than $\beta$. The associated eddy PV fluxes at depth are at least an order of magnitude smaller than in the upper level. There is no PV sheet in the bottom level because of the absence of a bottom Ekman layer in the three-dimensional flow. The Sverdrup solution gives a non-zero interior flow (in contrast to the channel experiments), and this dominates the flow in the bottom level. The vertical velocities in the interior of the gyres, arising through Ekman pumping and suction, are closed by opposing vertical flow in the western boundary currents where the Sverdrup vorticity balance no longer holds.

We go on now to study the eddy statistics choosing to focus on the upper level of the model where there are significant instantaneous and mean PV gradients and eddy fluxes of PV. 
(a)

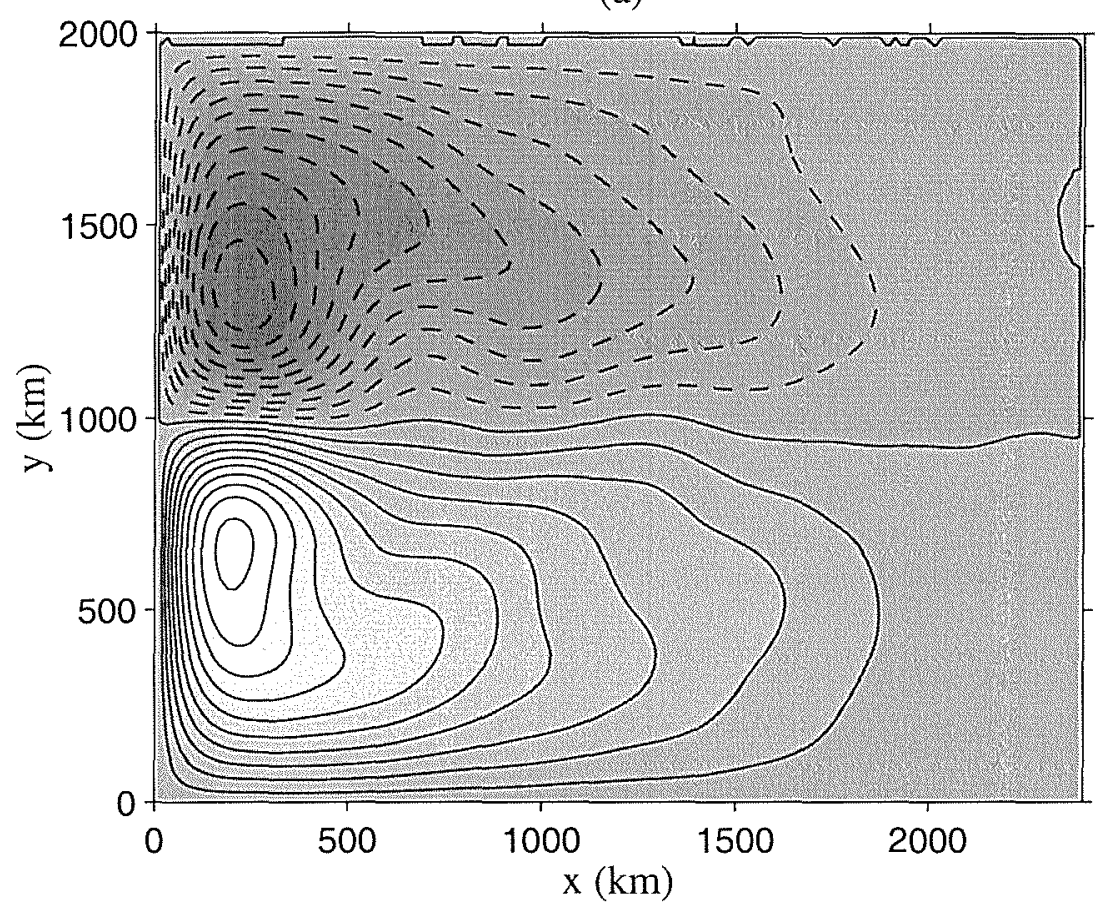

(b)

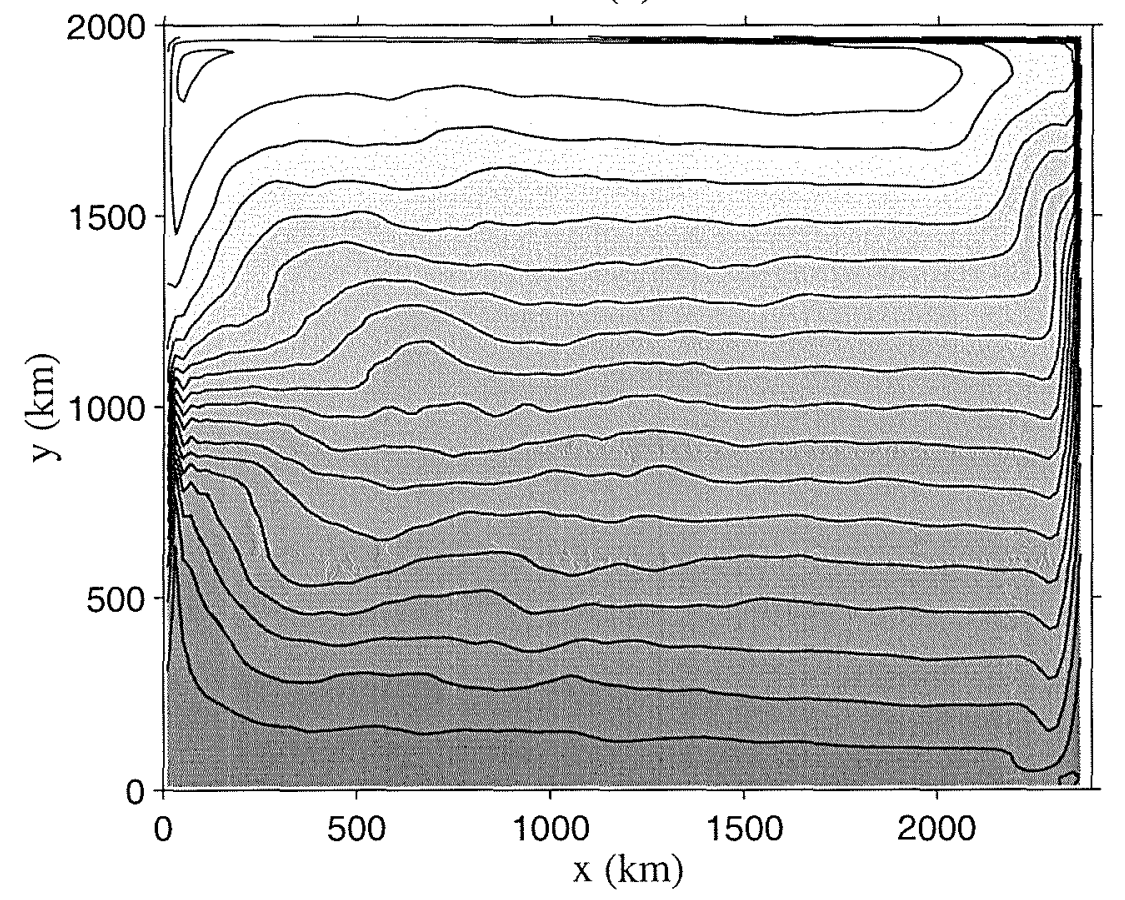

Figure 5.5: Fields of; (a) The time mean barotropic streamfunction $\bar{\psi}$. The range plotted is $\left(-40 \times 10^{6} \leq \bar{\psi} \leq 40 \times 10^{6}\right)$. The contour interval is $4 \times 10^{6}$. Units are $\mathrm{m}^{3} \mathrm{~s}^{-1}$. (b) The time mean surface (depth $=-50 \mathrm{~m}) \mathrm{PV} \bar{q}$. The range plotted is $\left(-3 \times 10^{-5} \leq \bar{q} \leq 2 \times 10^{-4}\right)$. The contour interval is $1 \times 10^{-5}$. Units are $s^{-2}$. 

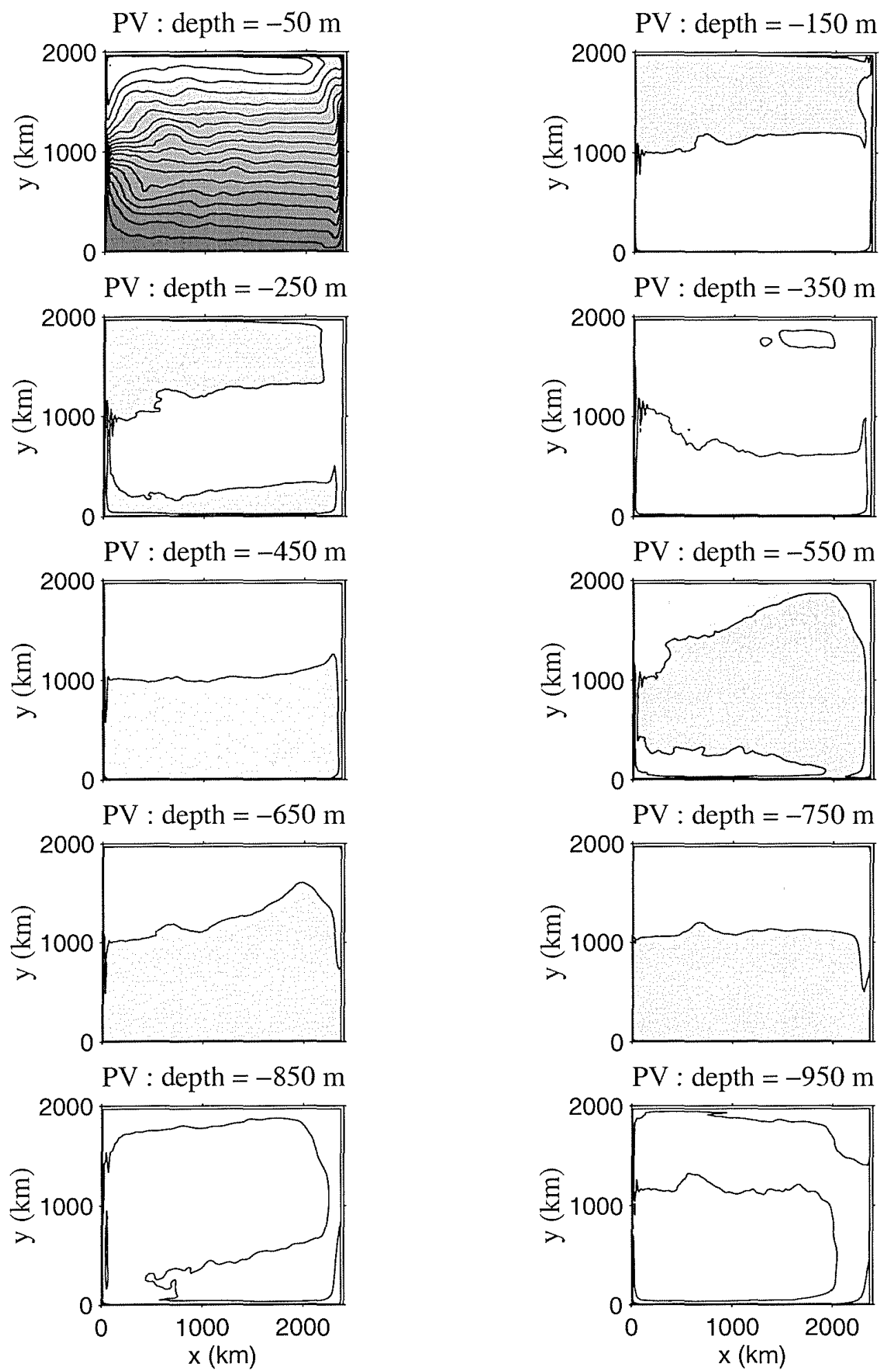

Figure 5.6: Plots of the mean $\mathrm{PV}, \bar{q}$, at each level of the model. The range plotted is $\left(-3 \times 10^{-5} \leq \bar{q} \leq 2 \times 10^{-4}\right)$. The contour interval is $1 \times 10^{-5}$. Units are $s^{-2}$. The $\mathrm{PV}$ sheet is evident in the upper level of the model. Weak PV gradients are present at every other level in the model. PV gradients in these levels are typically an order of magnitude smaller than the planetary vorticity gradient $\beta$. 


\subsubsection{Eddy transfer statistics}

The parameters that determine the flow, listed in Table 5.1, give surface eddy kinetic energy levels (figure 5.7) that are comparable in size to those observed in the upper ocean (see, for example, Wyrtki et al. (1976), Schmitz (1978), Richardson (1983)). The eddy

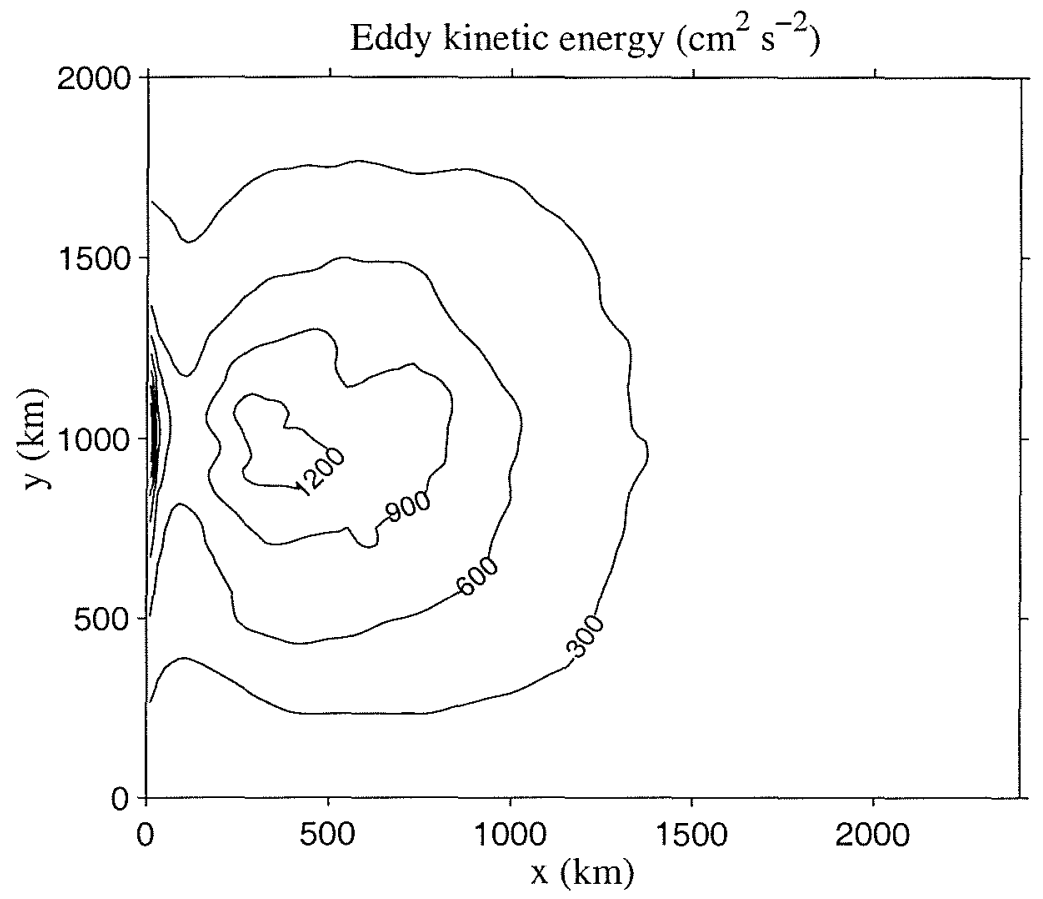

Figure 5.7: Time mean surface eddy kinetic energy field. Units are $\mathrm{cm}^{2} \mathrm{~s}^{-2}$.

kinetic energy penetrates into the basin interior with a maximum in intensity along the mean latitude of the jet. Figure 5.7 suggests the presence of a vigorous eddy field over much of the basin.

If our model had parameterized the eddies through the quasigeostrophic PV flux, the prognostic velocity variable would be that of the "residual mean circulation". Thus any comparison between an eddy resolving model and a parameterized simulation demands that we diagnose the residual mean circulation from the eddy resolving flow. Therefore, the residual mean circulation is diagnosed here. 


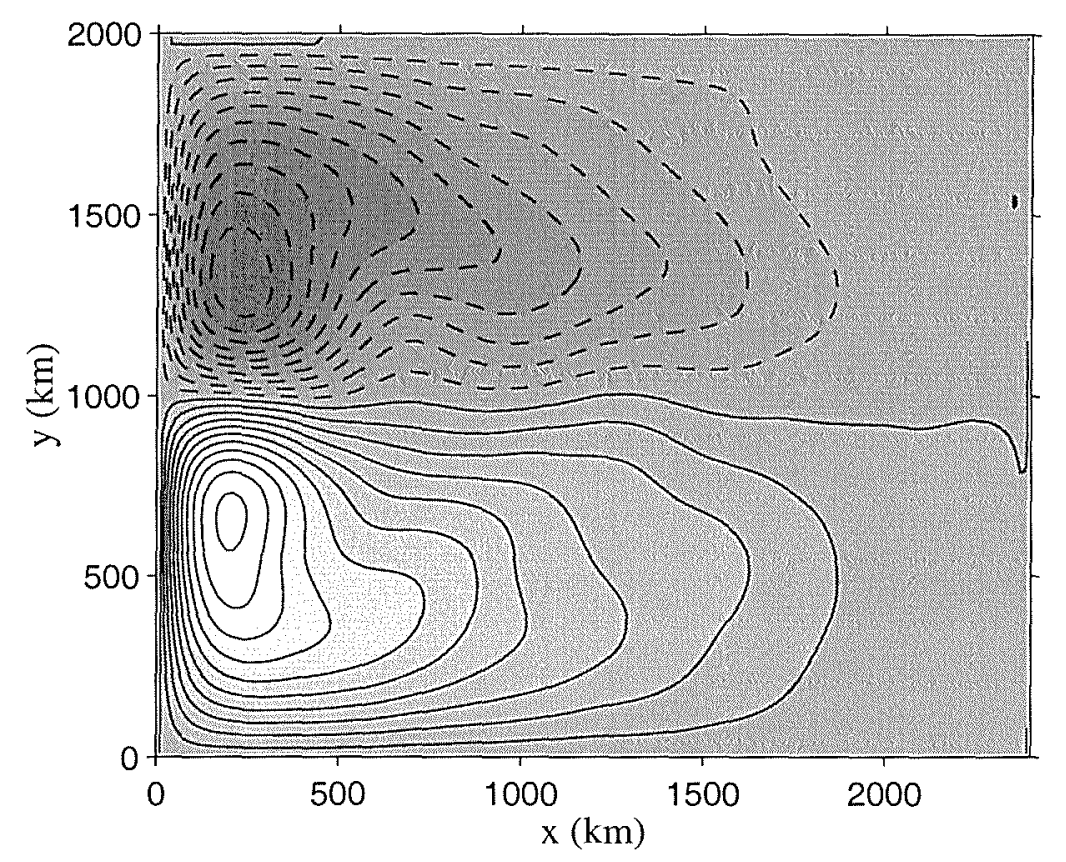

Figure 5.8: Time mean field of the barotropic residual mean streamfunction The range plotted is $\left(-40 \times 10^{6} \leq \bar{\psi} \leq 40 \times 10^{6}\right)$. The contour interval is $4 \times 10^{6}$. Units are $\mathrm{m}^{3} \mathrm{~s}^{-1}$.

The residual mean circulation defined by equation 2.31 can be written in component form:

$$
\begin{aligned}
\bar{u}^{\star} & =\bar{u}+\left[\frac{\partial}{\partial y}\left(\frac{1}{f}\left(\overline{u^{\prime 2}}+\overline{v^{\prime 2}}-\epsilon\right)\right)-\frac{\partial}{\partial z}\left(\overline{\frac{u^{\prime} T^{\prime}}{\overline{T_{z}}}}\right)\right], \\
\bar{v}^{\star} & =\bar{v}-\left[\frac{\partial}{\partial x}\left(\frac{1}{f}\left(\overline{u^{\prime 2}}+\overline{v^{\prime 2}}-\epsilon\right)\right)+\frac{\partial}{\partial z}\left(\overline{\frac{v^{\prime} T^{\prime}}{\overline{T_{z}}}}\right)\right], \\
\bar{w}^{\star} & =\bar{w}+\left[\frac{\partial}{\partial x}\left(\frac{\overline{u^{\prime} T^{\prime}}}{\overline{\bar{T}_{z}}}\right)+\frac{\partial}{\partial y}\left(\frac{\overline{v^{\prime} T^{\prime}}}{\overline{T_{z}}}\right)\right] .
\end{aligned}
$$

Algebraically simpler expressions for $\bar{u}^{\star}$ and $\bar{v}^{\star}$ can be found using the definitions of the PV fluxes (equations 2.14 and 2.15). The horizontal components are re-expressed thus:

$$
\bar{u}^{\star}=\bar{u}-\frac{1}{f_{0}}\left[\overline{u^{\prime} q^{\prime}}-\frac{\partial}{\partial x}\left(\overline{u^{\prime} v^{\prime}}\right)-\frac{\partial}{\partial y}\left(\overline{v^{\prime 2}}\right)\right],
$$




$$
\bar{v}^{\star}=\bar{v}-\frac{1}{f_{0}}\left[\overline{v^{\prime} q^{\prime}}+\frac{\partial}{\partial x}\left(\overline{u^{\prime 2}}\right)+\frac{\partial}{\partial y}\left(\overline{u^{\prime} v^{\prime}}\right)\right] .
$$

The depth-integrated residual mean streamfunction is plotted in figure 5.8. It looks very similar to the Eulerian mean streamfunction $\bar{\psi}$ shown in figure 5.5.a. However, there are $O\left(R_{o}\right)$ differences in the eddy intense region where the PV and Reynolds stress terms in equation 5.8 are important. The surface PV flux, $\overline{\left(\mathbf{u}^{\prime} q^{\prime}\right)}$, in the upper level (and therefore in the PV sheet) is plotted in figure 5.9. The figure also displays how the PV flux is oriented with respect to the mean gradients of PV. Areas shaded gray show regions of $\overline{\left(\mathbf{u}^{\prime} q^{\prime}\right)} \cdot \nabla \bar{q}<0$. Also shown are the contours of $\bar{q}$. The flux is large and down the mean PV gradient in the vicinity of the western wall where jet separation occurs and eddies are produced. West of $x=500 \mathrm{~km}$, dissipative effects are important, because of the enhanced gradients of eddy enstrophy, and act to drive the PV flux downgradient. In the region $(0 \leq x \leq 1000 \mathrm{~km}, 500 \mathrm{~km} \leq y \leq 1500 \mathrm{~km})$, the PV flux shows a cyclonic swirl which a strong component of the flux directed up the $\bar{q}$ gradient. This is because the eddy activity in this region is dominated by advection where the eddies spatially decay.

Regions of large counter-gradient fluxes of eddy PV suggest that a flux-gradient parameterization of the form of equation 3.5 is not appropriate for time mean flows. However, as we shall see in the next section, the decomposition of eddy fluxes into components that are associated with advective and dissipative effects, provides a way forward in which a relationship of the form of equation 3.5 may be justified. 
(a)

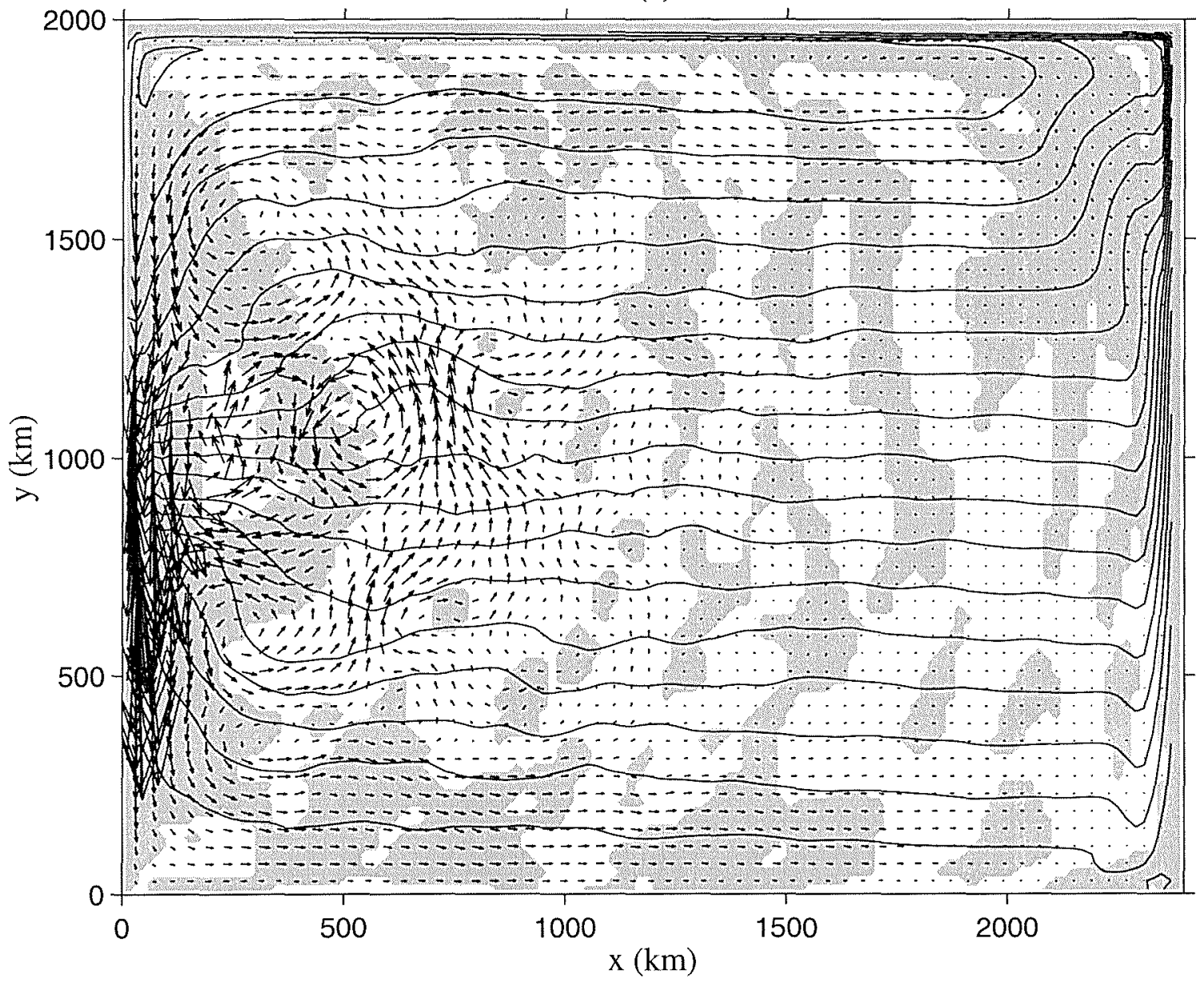

Figure 5.9: The eddy PV flux $\overline{\left(\mathbf{u}^{\prime} q^{\prime}\right)}$ superposed with the mean PV field $\bar{q}$. Every other vector is shown. The sign $\left(\overline{\left(\mathbf{u}^{\prime} q^{\prime}\right)} \cdot \nabla \bar{q}\right)$ is shaded. Gray indicates regions of downgradient PV transfer with $\overline{\left(\mathbf{u}^{\prime} q^{\prime}\right)} \cdot \nabla \bar{q}<0$. White indicates $\overline{\left(\mathbf{u}^{\prime} q^{\prime}\right)} \cdot \nabla \bar{q}>0$ or upgradient PV transfer. 


\subsubsection{Decomposition of the eddy flux of PV}

Figure 5.9 with its regions of upgradient PV transport highlights the difference between the time mean flow and the zonal mean flows of chapter 4 . The zonal mean meridional PV flux is naturally divergent and directed down the mean PV gradient. However, the time mean PV flux can have a large nondivergent (rotational) component that has no dynamical effect on the mean PV. This is because a flux of a quantity will only change the mean state if the flux is divergent.

Hoskins et al. (1983) studying atmospheric storm tracks, recognized this and removed the nondivergent portion of the eddy flux making heuristic assumptions about the size and shape of the eddies. First, they assumed the relative length scales of the eddies were such that $\left(\partial_{y} \gg \partial_{x}\right)$. Secondly, the specified the relative size of the different momentum fluxes to be $\overline{v^{\prime 2}}$ and $\overline{v^{\prime 2}} \gg \overline{u^{\prime} v^{\prime}}$. The resultant eddy flux that they obtained was an extension of the Eliassen-Palm flux for zonal flows which they used as a diagnostic of the feedback of the eddies on the mean flow. The assumptions employed by Hoskins et al. are not appropriate for the flows considered in this chapter. An alternative approach was offered by Holopainen (1984) in which he defined an eddy time mean force in the atmosphere to be given by $-\mathbf{k} \times{\overline{\left(\mathbf{u}^{\prime} q^{\prime}\right)}}_{D i v}$. He estimates ${\overline{\left(\mathbf{u}^{\prime} q^{\prime}\right)}}_{D i v}$ by solving the Poisson equation for its potential function.

The decomposition of the eddy PV flux into divergent and rotational parts is a problematic feature of time mean eddy statistics. A requisite for the method used by

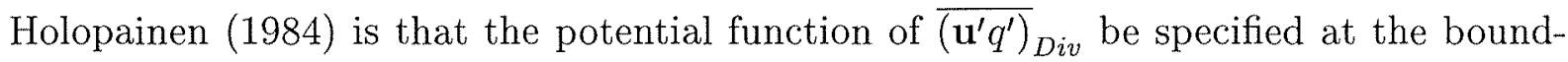
aries. This is a difficult task and as a result this approach has proved to be of little use in analyses of ocean eddies. Therefore, alternative techniques have been used to determine the flux components using analytical methods.

Marshall and Shutts (1981) and Illari and Marshall (1983) demonstrate that if the time mean flow is almost conservative $(\overline{\mathbf{u}} \cdot \nabla \bar{q} \approx 0)$, there is a purely rotational component of $\overline{\mathbf{u}^{\prime} q^{\prime}}$ associated with mean advection of eddy PV variance or enstrophy. This rotational 
flux is defined thus

$$
{\overline{\left(\mathbf{u}^{\prime} q^{\prime}\right)_{R o t}}}_{\text {R }} \times \mathbf{k}(\Lambda e),
$$

where

$$
\Lambda=\frac{d \bar{\psi}}{d \bar{q}}=\frac{\nabla \bar{q} \cdot \nabla \bar{\psi}}{|\nabla \bar{q}|^{2}},
$$

and the eddy enstrophy, e, is given by

$$
e=\frac{1}{2} \overline{q^{\prime 2}} .
$$

If $\overline{\mathbf{u}} \cdot \nabla \bar{q}=0$ holds, then $J(\bar{\psi}, \bar{q})=0$, and $\bar{\psi}=F(\bar{q})$ where $F$ is a functional relationship

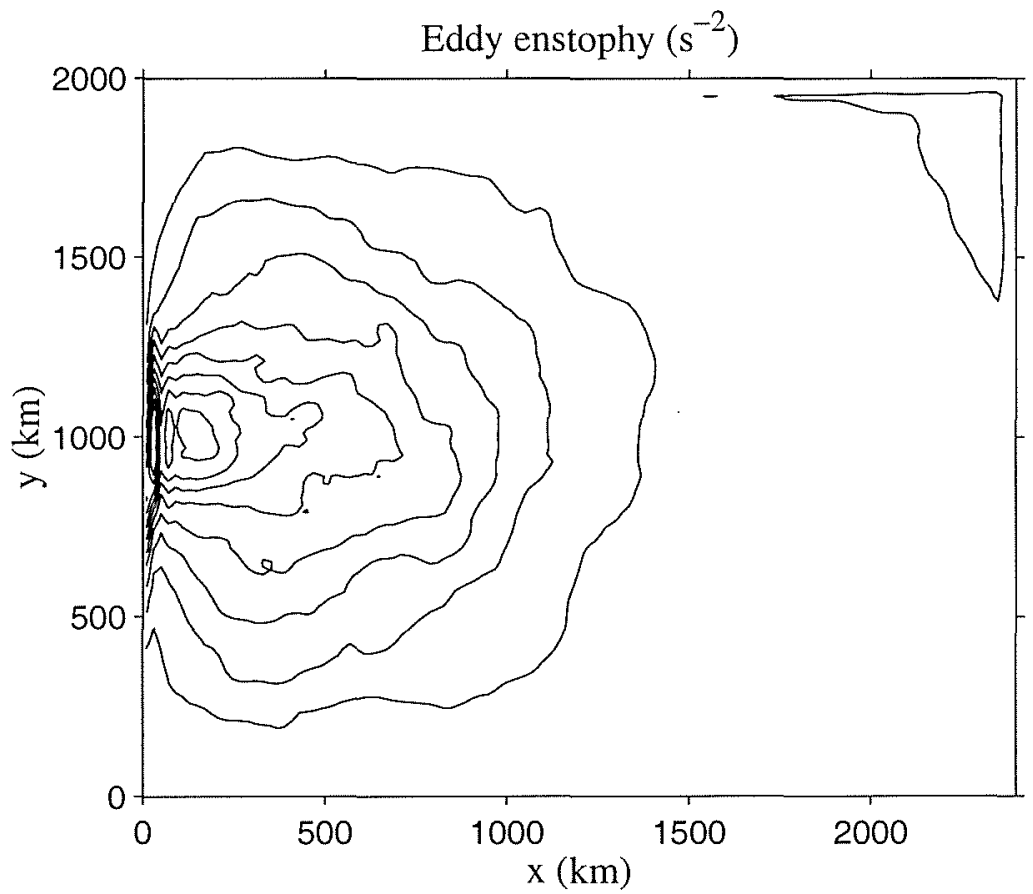

Figure 5.10: Time mean surface field of eddy enstrophy e. The contour interval is $2.5 \times 10^{-10} \mathrm{~s}^{-2}$.

between $\bar{\psi}$ and $\bar{q} . F$ is usually determined empirically. Thus we can use the following relationship to calculate $d \bar{\psi} / d \bar{q}$ :

$$
\frac{d \bar{\psi}}{d \bar{q}}=\operatorname{sign}(\nabla \bar{q} \cdot \nabla \bar{\psi}) \frac{|\nabla \bar{\psi}|}{|\nabla \bar{q}|} .
$$


The time mean eddy enstrophy is plotted in figure 5.10 for the upper level of the model. It is similar to the map of eddy kinetic energy with contours straddling the jet to the north and south. It has a maximum at the western boundary associated with the vacillation of the separation point of the jet from the western wall. The enstrophy penetrates (at this contour interval) to half of the zonal basin extent and almost the whole extent in latitude. Also present in the northeastern contour is a variance contour associated with the "northeastern feature" given by the C-grid. Note that the enstrophy connected with the northeastern feature is detached from the enstrophy contours associated with the eddying jet. Thus the presence of the northeastern feature does not influence the results in the jet region.

The sign of $\nabla \bar{q} \cdot \nabla \bar{\psi}$, (and to the extent that $\bar{\psi}$ and $\bar{q}$ are parallel, the sign of $d \bar{\psi} / d \bar{q}$ ), is calculated and plotted in figure 5.11. The $\bar{\psi}$ contours are wavy in the region where

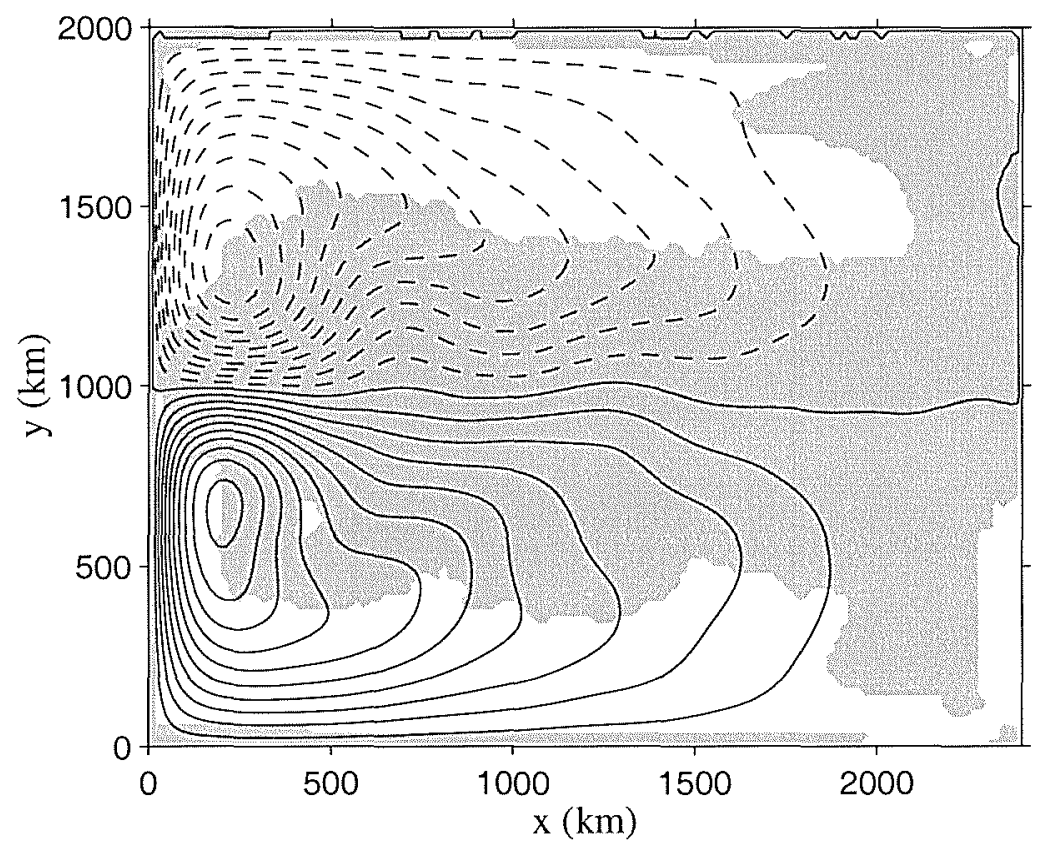

Figure 5.11: Time mean surface field of the sign $(\nabla \bar{q} \cdot \nabla \bar{\psi})$ and Eulerian mean barotropic streamfunction. Regions shaded in gray indicate $\nabla \bar{q} \cdot \nabla \bar{\psi}<0$. Regions shaded white have $\nabla \bar{q} \cdot \nabla \bar{\psi}>0$. 
$\nabla \bar{q} \cdot \nabla \bar{\psi}<0$. This is because of the presence of standing waves. The direction $\nabla \bar{q}$ is that in which Rossby waves propagate their phase, while the mean flow is in the direction of $\nabla \bar{\psi}$. If these gradients are opposed then standing waves can result, producing waviness in the $\bar{\psi}$ contours.

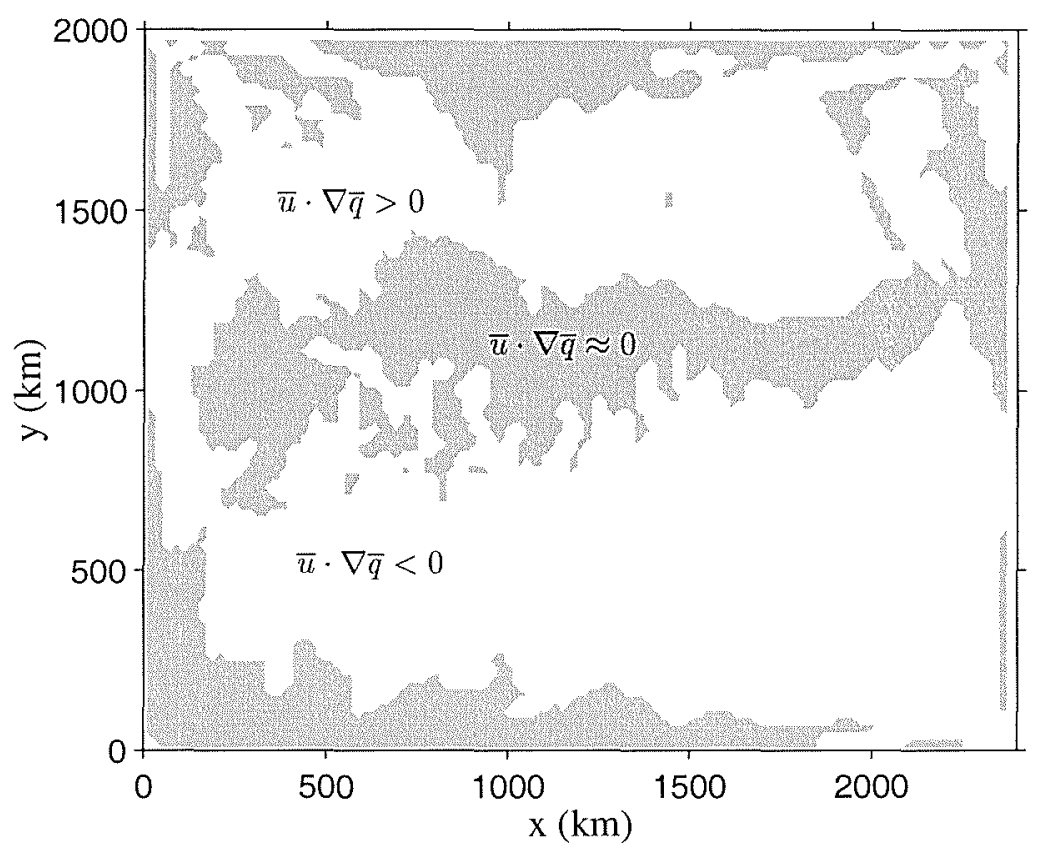

Figure 5.12: Plot of $\bar{u} \cdot \nabla \bar{q}$. Gray areas indicate regions where the time mean flow is nearly conservative, $\bar{u} \cdot \nabla \bar{q} \approx 0$. The close conservation of $\bar{q}$ in the jet region enables rationalization of the eddy PV flux components that are associated with advective and dissipative effects. The eddy flux component balancing mean flow advection is nondivergent and does not force the mean flow. The eddy flux component that contains all of the divergent flux is directed down the mean $\bar{q}$ gradient and plays the role of balancing the dissipation of eddy enstrophy White areas indicate regions of non-conservation of $\bar{q}$ where the mean flow is driven across $\bar{q}$ contours by the wind stress curl.

The result of the definition of the rotational component of the PV flux given by equation 5.9 , is that this flux will balance the mean advection of eddy enstrophy:

$$
\overline{\mathbf{u}} \cdot \nabla e=-{\overline{\left(\mathbf{u}^{\prime} q^{\prime}\right)_{R o t}}}_{R \bar{q}} .
$$

Further, if triple correlation terms are small, the residual flux between the full PV flux 
and the rotational component (termed here ${\overline{\left(\mathbf{u}^{\prime} q^{\prime}\right)}}_{\text {Div }}$ ),

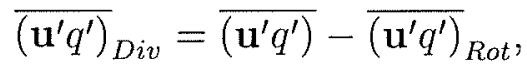

will balance sources and sinks of eddy enstrophy, $\overline{\chi^{\prime} q^{\prime}}$ :

$$
{\overline{\left(\mathbf{u}^{\prime} q^{\prime}\right)_{D i v}}} \cdot \nabla \bar{q}=\overline{\chi^{\prime} q^{\prime}}
$$

The sign of $\overline{\chi^{\prime} q^{\prime}}$ determines whether the divergent flux is directed up or down the mean PV gradient. If the non-conservative term is a dissipation of eddy enstrophy via the enstrophy cascade, then $\overline{\chi^{\prime} q^{\prime}}<0$, and the divergent flux will point down the $\bar{q}$ gradient.

In actuality, the divergent flux defined by equation 5.14 need not be purely divergent,

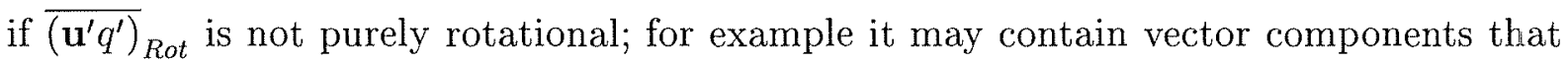
are uniform or translational (Bluestein (1993)). However, Plumb (1986) shows that the eddy PV flux body force associated with the residual PV flux (in our notation: $\left.\mathbf{F}_{\text {Div }}=-\mathbf{k} \times{\overline{\left(\mathbf{u}^{\prime} q^{\prime}\right)_{D i v}}}\right)$ can be related to a generalized Eliassen-Palm flux $\mathbf{M}_{\mathrm{T}}$. We now define a unit vector which is directed along the mean PV (and streamfunction) gradient,

$$
\mathbf{n}=\frac{\nabla \bar{q}}{|\nabla \bar{q}|}
$$

and one which is oriented along the mean PV contours,

$$
\mathbf{s}=\mathbf{k} \times \mathbf{n}
$$

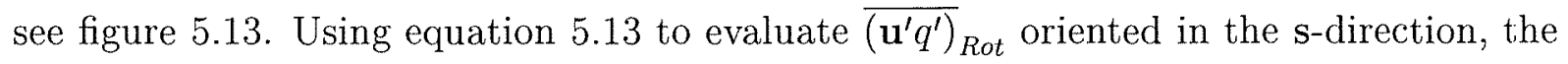
eddy force in the direction of the flow can be written as

$$
\begin{aligned}
\mathbf{F}_{\text {Div }} \cdot \mathbf{s} & =\overline{\left(\mathbf{u}^{\prime} q^{\prime}\right)} \cdot \mathbf{n}+\frac{1}{\nabla \bar{q}} \overline{\mathbf{u}} \cdot \nabla e \\
& =\nabla \cdot \mathbf{M}_{\mathbf{T}}
\end{aligned}
$$

Hence, $\mathbf{M}_{\mathbf{T}}$ is an effective flux of momentum, measured in the direction of the flow (for small-amplitude disturbances of a slowly varying, and nearly conservative mean flow). 
The momentum flux $\mathbf{M}_{\mathbf{T}}$ is independent of $d \bar{\psi} / d \bar{q}$ and can be related to a wave activity flux:

$$
\frac{\partial}{\partial t}\left(\frac{e}{\nabla \bar{q}}\right)+\nabla \cdot \mathbf{M}_{\mathbf{T}}=\mathcal{D} .
$$

This is a time mean wave activity relation, that is identical to equation 2.67 for zonal mean flows. Hence, $\mathbf{M}_{\mathbf{T}}$ can be interpreted as a measure of eddy activity in the along flow direction.

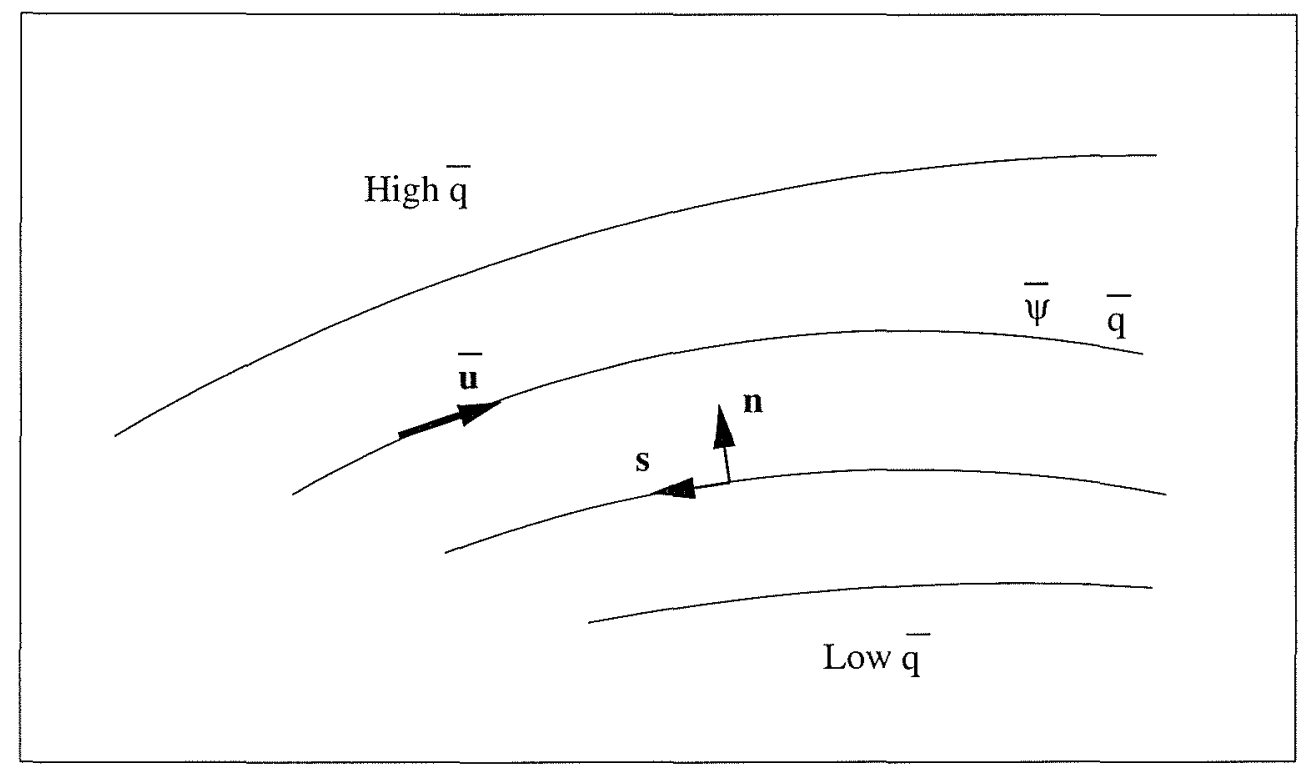

Figure 5.13: The unit vectors $\mathbf{n}$ and $\mathbf{s . ~} \mathbf{n}=\frac{\nabla \bar{q}}{|\nabla \bar{q}|}$ is directed along the mean PV gradient. $\mathbf{s}=\mathbf{k} \times \mathbf{n}$ is directed in the upstream direction. Adapted from Plumb (1986).

In figure 5.14, the rotational flux in the upper level of the model, $\left.\overline{\left(\mathbf{u}^{\prime} q^{\prime}\right.}\right)_{R o t}$ is superposed on the contours of eddy enstrophy. It is mostly directed up the mean gradient of $\bar{q}$ because it plays the role of balancing the mean flow advection of enstrophy in the jet region where $\overline{\mathbf{u}} \cdot \nabla e<0$. Figure 5.17.b plots this flux in the subdomain $0 \leq x \leq 1000 \mathrm{~km}$, $500 \mathrm{~km} \leq y \leq 1500 \mathrm{~km}$, (hereafter subdomain A), which highlights how the rotational flux follows the enstrophy contours.

Figure 5.15 plots the corresponding upper level component of the divergent flux

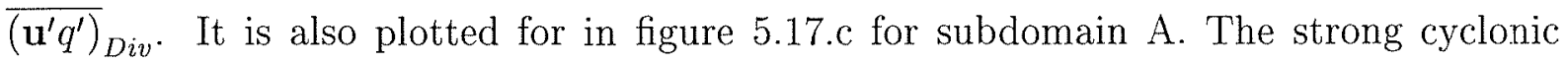
swirl evident in the full PV flux is no longer present. It has been largely accounted for by 


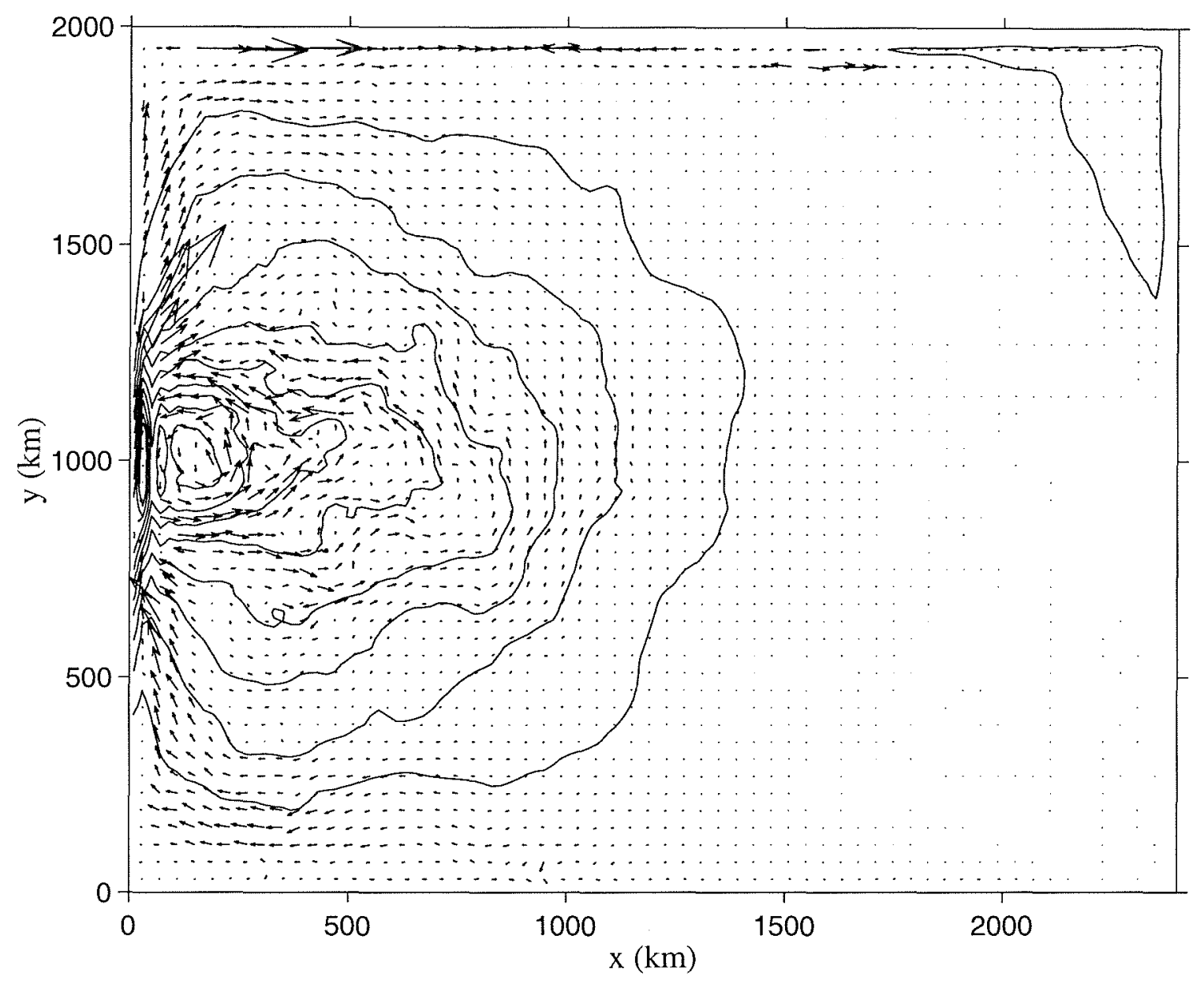

Figure 5.14: The eddy PV flux ${\overline{\left(\mathbf{u}^{\prime} q^{\prime}\right)}}_{\text {Rot }}$ superposed with the contours of eddy enstrophy, e. Every other vector is shown.

the rotational flux. Regions of enstrophy sources and sinks can be readily identified according to whether the divergent flux is directed up or down the mean PV gradient. Over most of the basin the component of ${\overline{\left(\mathbf{u}^{\prime} q^{\prime}\right)}}_{\text {Div }}$ is directed along the gradient is vanishingly small. The exception to this is in the eddy intense jet region where strong downgradient fluxes exist because it is a region of intense enstrophy and a weakened potential vorticity gradients. Also shown is the sign of ${\overline{\left(\mathbf{u}^{\prime} q^{\prime}\right)}}_{D i v} \cdot \nabla \bar{q}$ with gray shading indicating negative 


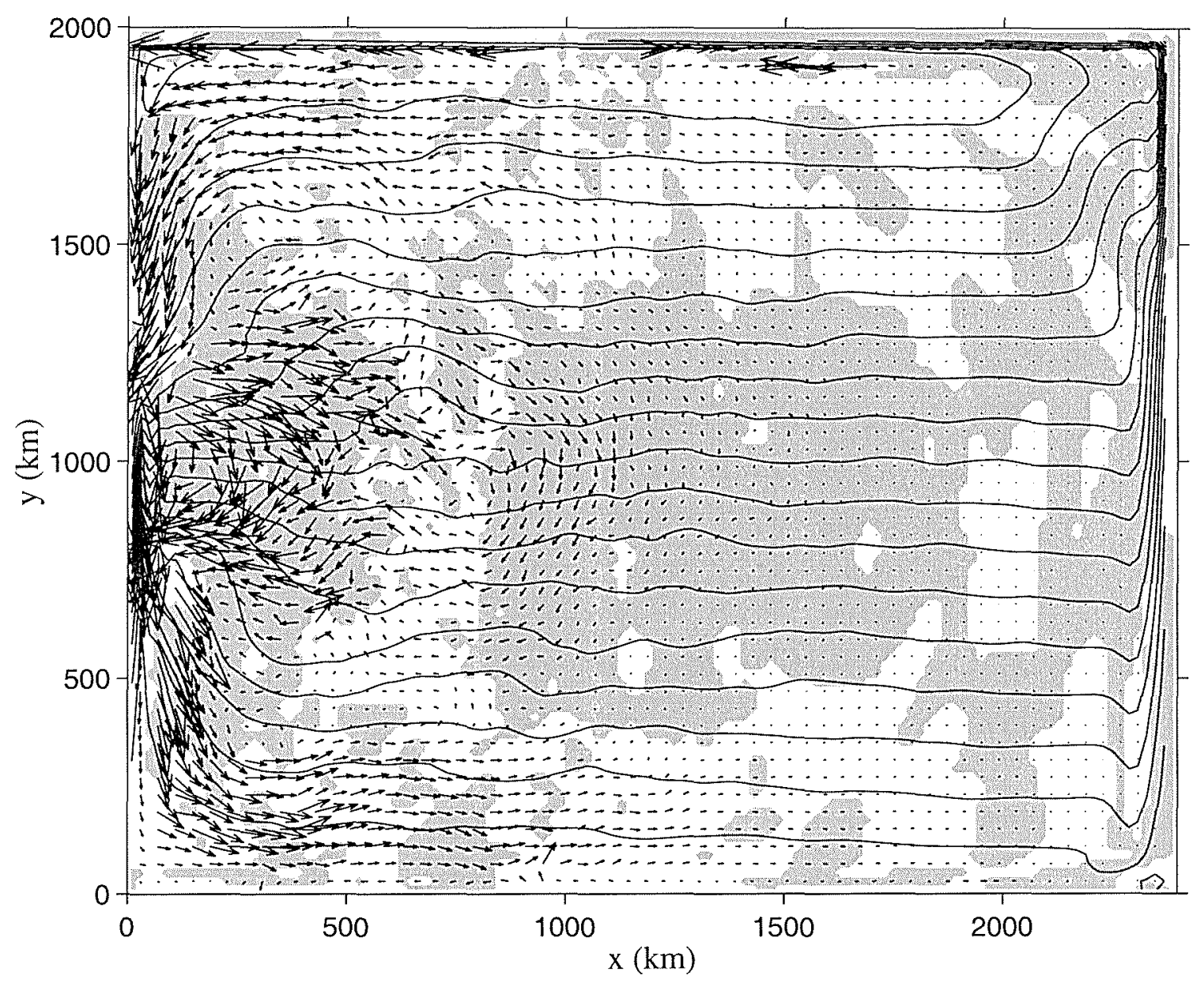

Figure 5.15: The eddy PV flux $\overline{\left(\mathbf{u}^{\prime} q^{\prime}\right)_{\text {Rot }}}$ superposed with the contours of eddy enstrophy, e. Every other vector is shown. The sign $\left({\overline{\left(\mathbf{u}^{\prime} q^{\prime}\right)}}_{D i v} \cdot \nabla \bar{q}\right)$ is shaded. Gray indicates regions of $\overline{\left(\mathbf{u}^{\prime} q^{\prime}\right)_{D i v}} \cdot \nabla \bar{q}<0$. White indicates $\overline{\left(\mathbf{u}^{\prime} q^{\prime}\right)_{D i v}} \cdot \nabla \bar{q}>0$.

values and downgradient transfer of $\bar{q}$ by the divergent flux ${\overline{\left(\mathbf{u}^{\prime} q^{\prime}\right)}}_{D i v}$. Note that in figure 5.15, almost all regions of significant ${\overline{\left(\mathbf{u}^{\prime} q^{\prime}\right)}}_{D i v}$ correspond to regions of downgradient transfer of PV.

There are however, regions where $\overline{\left(\mathbf{u}^{\prime} q^{\prime}\right)}$ Div $\cdot \nabla \bar{q}>0$ as indicated by the white shading. In these areas, PV transfer is directed up the mean PV gradient. This occurs at latitudes 
where the mean flow is meridional and turning westward in the gyres. It arises because $d \bar{\psi} / d \bar{q}>0$ at these latitudes as figure 5.11 shows. However, it is at this point in the flow that the validity of equation 5.12 is in doubt, because $J(\bar{\psi}, \bar{q}) \neq 0$ (see figure 5.12). This is because the mean flow is being driven across $\bar{q}$ contours by the curl of the wind stress. There is a region of strong divergent flux in the southwestern corner of the basin. This is due to large values of the analytically determined rotational flux which arises for two reasons. Firstly, $\bar{u} \cdot \bar{q} \approx 0$ is not valid in this region (figure 5.12) because of the forcing by the wind stress curl, as mentioned above. Secondly, in this region, the value of $d \bar{\psi} / d \bar{q}$ is relatively large due to weakened meridional gradients of mean PV (figure 5.5). Figure 5.15 also shows a region just to the east of the jet around $x=500 \mathrm{~km}$ where the divergent flux is upgradient. However, inspection of figure 5.17 shows that in this region, the along gradient component of ${\overline{\left(\mathbf{u}^{\prime} q^{\prime}\right)}}_{D i v}$ is vanishingly small when compared to the cross stream flux in the eddy intense regions west of $x=500 \mathrm{~km} . \quad \overline{\left(\mathbf{u}^{\prime} q^{\prime}\right)} \cdot \nabla \bar{q}$ and $\overline{\left(\mathbf{u}^{\prime} q^{\prime}\right)_{D i v}} \cdot \nabla \bar{q}$ are

(a)

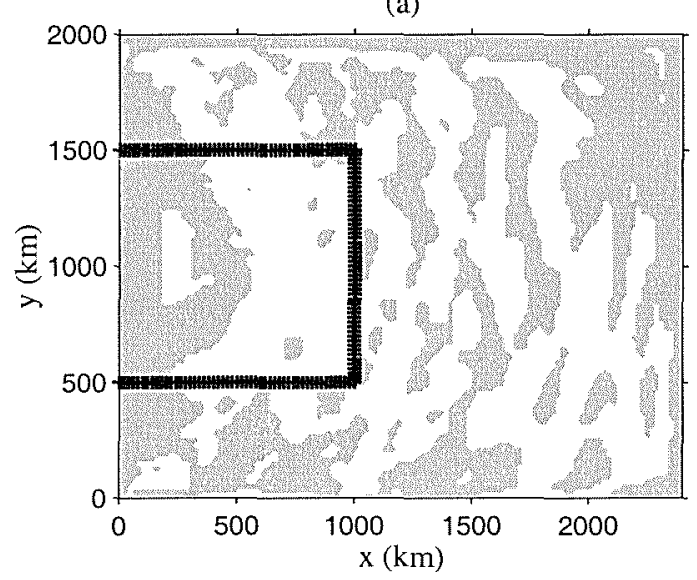

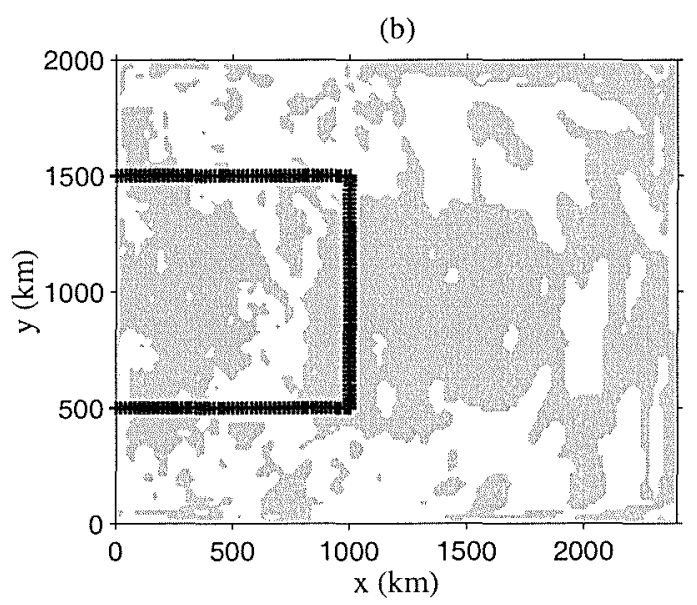

Figure 5.16: (a) The sign $\left(\overline{\left(\mathbf{u}^{\prime} q^{\prime}\right)} \cdot \nabla \bar{q}\right)$. (b) The $\operatorname{sign}\left({\overline{\left(\mathbf{u}^{\prime} q^{\prime}\right)}}_{D i v} \cdot \nabla \bar{q}\right)$. Areas shaded in gray are regions where the quantity is negative indicating downgradient PV transfer. Subdomain A is indicated. Within the subdomain, white areas correspond to to regions of upgradient PV transfer. However, as figure 5.17 shows, the white areas in (b) correspond to regions where the upgradient flux in vanishingly small and thus have little effect on the mean flow. 
(a)

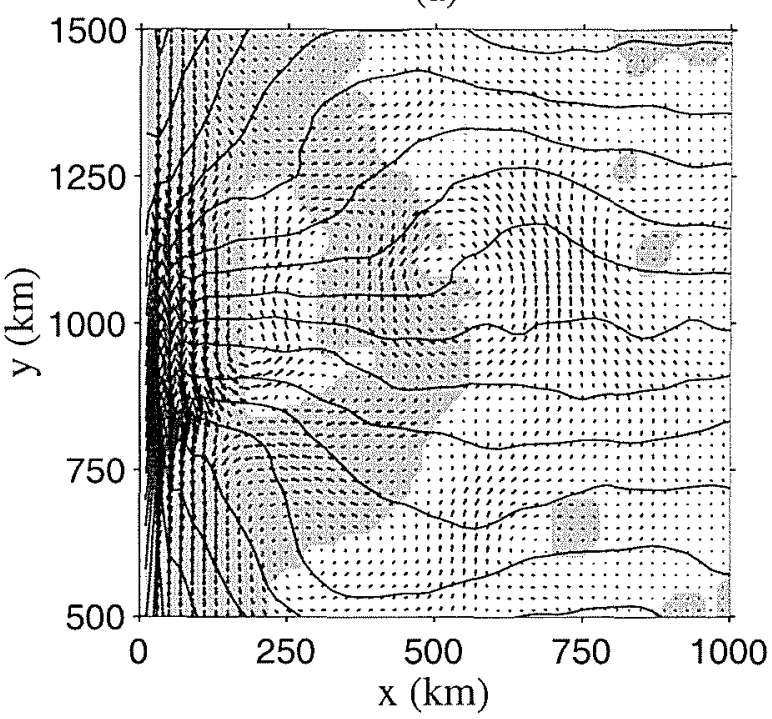

(b)

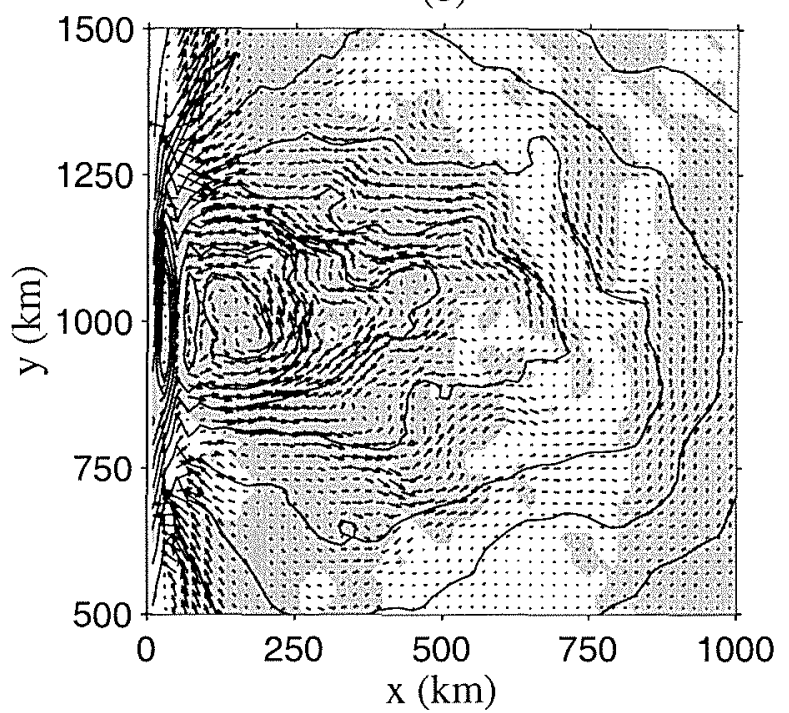

(c)

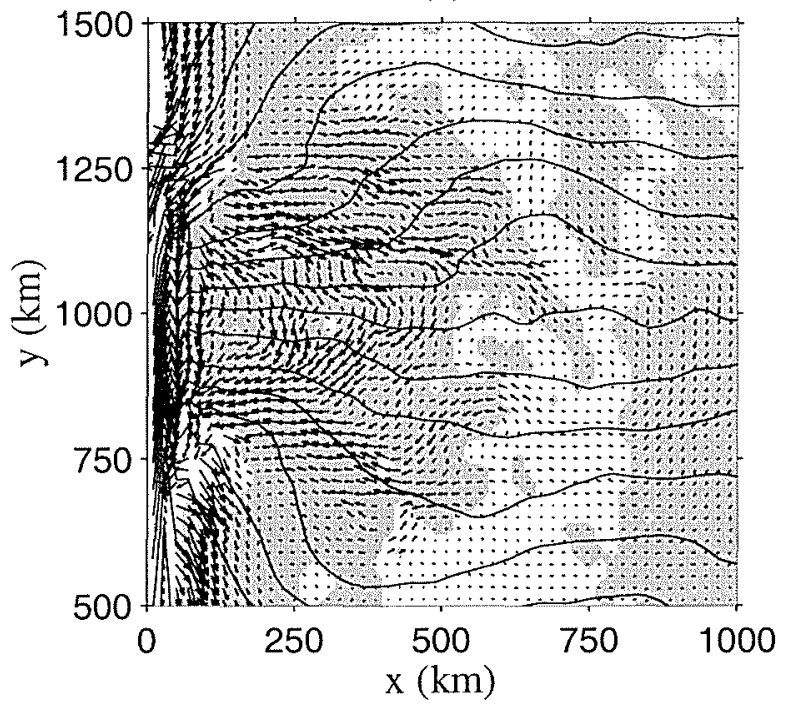

Figure 5.17: The upper level eddy fluxes; (a) $\overline{\left(\mathbf{u}^{\prime} q^{\prime}\right)}$ superposed on the $\operatorname{sign}\left(\overline{\left(\mathbf{u}^{\prime} q^{\prime}\right)} \cdot \nabla \bar{q}\right)$.

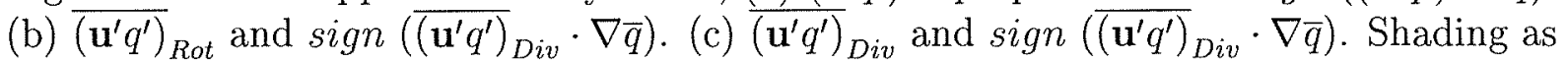
before.

redrawn in figures 5.16.a and b. We see that in subdomain $\mathrm{A}$, the region of intense eddy driving of the mean flow, the area of upgradient $\overline{\left(\mathbf{u}^{\prime} q^{\prime}\right)}$ transfer is smaller than in the full 
PV flux, and from figure 5.17 the along gradient component of the flux is negligible.

Figure 5.18 plots a scatter diagram of $\bar{\psi}$ against $\bar{q}$ based on the points in subdomain A. The figure shows a strong $\bar{q}-\bar{\psi}$ relationship, particularly at midlatitudes. Thus we have confidence in the size and structure of the analytically calculated $\overline{\left(\mathbf{u}^{\prime} q^{\prime}\right)}$ Rot field in the eddy intense region associated with the jet.

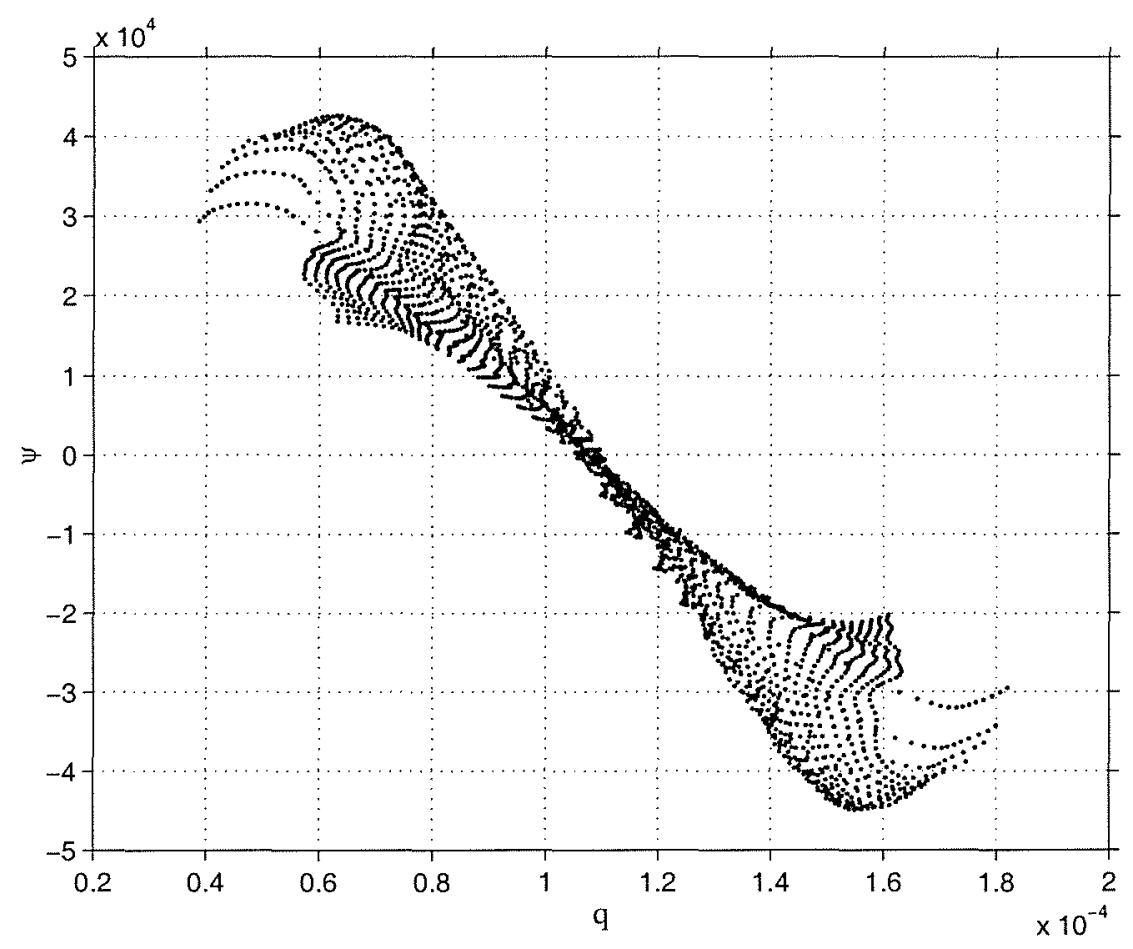

Figure 5.18: Scatter diagram for mean PV $\bar{q}$ against mean streamfunction $\bar{\psi}$ for the region $0 \leq x \leq 1000 \mathrm{~km}, 500 \leq y \leq 1500 \mathrm{~km}$.

In summary, this section has shown that the conceptual framework of downgradient PV mixing is still appropriate for the component of the eddy PV flux that forces the mean flow. This was determined through consideration of the dynamical balances in the eddy enstrophy equation. Assuming close conservation of $\bar{q}$ enables rationalization of the eddy PV flux. A rotational, non-divergent eddy flux component is associated with the mean flow advection of eddy enstrophy. This component, associated with advective effects, does not force the mean flow because it is non-divergent. The residual, ${\overline{\left(\mathbf{u}^{\prime} q^{\prime}\right)_{D i v}}}=$ 


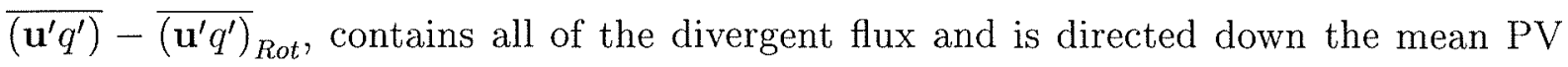
gradient due to enstrophy dissipation.

\subsection{Representation of the time mean eddy-forcing}

In the previous section, we found that in the regions of significant eddy forcing, the divergent component of the eddy flux ${\overline{\left(\mathbf{u}^{\prime} q^{\prime}\right)}}_{\text {Div }}$ was directed down the mean gradient of $\bar{q}$. Hence a parameterization of downgradient PV transfer in the form of equation 3.5 seems to be justified if ${\overline{\left(\mathbf{u}^{\prime} q^{\prime}\right)}}_{\text {Div }}$ is represented instead of the full flux $\overline{\left(\mathbf{u}^{\prime} q^{\prime}\right)}$. This is not surprising given the diagnostic studies of Hoskins et al. (1983) and Holopainen (1984) referred to in the previous section. Indeed, working within the context of a barotropic quasigeostrophic model, Marshall (1984) made the same proposal. However, our governing equations are not quasigeostrophic, only the eddy terms are. If we are to invoke a parameterization of the form

$$
{\overline{\left(\mathbf{u}^{\prime} q^{\prime}\right)}}_{D i v}=-\mathbf{K} \cdot \nabla \bar{q}
$$

then the full PV flux term that appears in equation 2.55.a must be replaced by a term that depends only on the divergent PV flux. Thus we proceed by removing the rotational (nondivergent) component of the PV flux from $\bar{F}_{\text {eddies }}=-\mathbf{k} \times \overline{\left(\mathbf{u}^{\prime} q^{\prime}\right)}$.

First, we note that:

$$
\begin{aligned}
\mathbf{k} \times{\overline{\left(\mathbf{u}^{\prime} q^{\prime}\right)_{R o t}}} & =\mathbf{k} \times \mathbf{k} \times \nabla(\Lambda e) \\
& =-\nabla(\Lambda e)
\end{aligned}
$$

Now, using equation 5.14 for the divergent component of the flux,

$$
\begin{aligned}
-\mathbf{k} \times{\overline{\left(\mathbf{u}^{\prime} q^{\prime}\right)_{D i v}}} & =-\mathbf{k} \times\left[\overline{\mathbf{u}^{\prime} q^{\prime}}-{\overline{\left(\mathbf{u}^{\prime} q^{\prime}\right)_{R o t}}}\right] \\
& =-\mathbf{k} \times \overline{\mathbf{u}^{\prime} q^{\prime}}+\mathbf{k} \times{\overline{\left(\mathbf{u}^{\prime} q^{\prime}\right)_{R o t}}} \\
& =-\mathbf{k} \times \overline{\mathbf{u}^{\prime} q^{\prime}}-\nabla(\Lambda e)
\end{aligned}
$$


so we have

$$
-\mathbf{k} \times \overline{\mathbf{u}^{\prime} q^{\prime}}=-\mathbf{k} \times{\overline{\left(\mathbf{u}^{\prime} q^{\prime}\right)}}_{D i v}+\nabla(\Lambda e)
$$

Using equation 5.23 to substitute for the body force term in equation 2.55.a we obtain:

$$
\frac{D^{\star} \overline{\mathbf{u}}^{\star}}{D t}+f \mathbf{k} \times \overline{\mathbf{u}}^{\star}+\frac{1}{\rho_{0}} \nabla \bar{p}=\overline{\mathbf{F}}-\mathbf{k} \times{\overline{\left(\mathbf{u}^{\prime} q^{\prime}\right)}}_{D i v}+\nabla(\Lambda e) .
$$

It readily follows that,

$$
\frac{D^{\star} \overline{\mathbf{u}}^{\star}}{D t}+f \mathbf{k} \times \overline{\mathbf{u}}^{\star}-\nabla(\Lambda e)+\frac{1}{\rho_{0}} \nabla \bar{p}=\overline{\mathbf{F}}-\mathbf{k} \times{\overline{\left(\mathbf{u}^{\prime} q^{\prime}\right)}}_{D i v}
$$

Absorbing $\nabla(\Lambda e)$ into the Coriolis term yields

$$
\frac{D^{\star} \overline{\mathbf{u}}^{\star}}{D t}+f \mathbf{k} \times\left[\overline{\mathbf{u}}^{\star}-\frac{1}{f} \mathbf{k} \times \nabla(\Lambda e)\right]+\frac{1}{\rho_{0}} \nabla \bar{p}=\overline{\mathbf{F}}-\mathbf{k} \times{\overline{\left(\mathbf{u}^{\prime} q^{\prime}\right)_{D i v}}} .
$$

We now choose to redefine the residual mean velocity thus:

$$
\overline{\mathbf{u}}^{\star \star}=\overline{\mathbf{u}}^{\star}-\frac{1}{f} \mathbf{k} \times \nabla(\Lambda e) .
$$

So we have

$$
\frac{D^{\star} \overline{\mathbf{u}}^{\star}}{D t}+f \mathbf{k} \times \overline{\mathbf{u}}^{\star \star}+\frac{1}{\rho_{0}} \nabla \bar{p}=\overline{\mathbf{F}}-\mathbf{k} \times{\overline{\left(\mathbf{u}^{\prime} q^{\prime}\right)}}_{D i v}
$$

The rotational component of the PV flux has been absorbed into the Coriolis term. The result is that a nondivergent eddy force term appears that is dependent only in the divergent component of the eddy PV flux. As equation 5.28 stands the velocities that appear are a mixture of the residual mean velocities $\overline{\mathbf{u}}^{\star}$ and the velocity $\overline{\mathbf{u}}^{\star \star}$. This is useful if equation 5.28 were to be used diagnostically. However, if we wish to solve for the mean flow in a predictive model in which the eddy fluxes are parameterized, then to utilize equation 5.28 demands that we diagnose the rotational component of the PV flux in order to evaluate $\overline{\mathbf{u}}^{\star \star}$. Thus the obvious way forward is to form a momentum equation in which the prognostic velocity is $\overline{\mathbf{u}}^{\star \star}$. This can be achieved without much difficulty through manipulation of the substantial derivative and systematic scaling as carried out in chapter 2 . 
Transforming the advective velocity in the substantial derivative from $\overline{\mathbf{u}}^{\star}$ to $\overline{\mathbf{u}}^{\star \star}$ yields:

$$
\begin{aligned}
\frac{D^{\star \star} \overline{\mathbf{u}}^{\star \star}}{D t}+f \mathbf{k} \times \overline{\mathbf{u}}^{\star \star}+\frac{1}{\rho_{0}} \nabla \bar{p} & =\overline{\mathbf{F}}-\mathbf{k} \times{\overline{\left(\mathbf{u}^{\prime} q^{\prime}\right)}}_{D i v}+\frac{D^{\star \star}}{D t}\left(\frac{1}{f} \mathbf{k} \times \nabla(\Lambda e)\right) \\
& -\left(\frac{1}{f} \mathbf{k} \times \nabla(\Lambda e)\right) \cdot \nabla\left[\overline{\mathbf{u}}^{\star \star}-\frac{1}{f} \mathbf{k} \times \nabla(\Lambda e)\right]
\end{aligned}
$$

For algebraic simplicity we write

$$
\frac{1}{f} \mathbf{k} \times \nabla(\Lambda e)=\frac{1}{f}{\overline{\left(\mathbf{u}^{\prime} q^{\prime}\right)}}_{R o t}
$$

with which the right hand side of equation 5.29 can be rewritten as

$$
R H S=\overline{\mathbf{F}}-\mathbf{k} \times{\overline{\left(\mathbf{u}^{\prime} q^{\prime}\right)_{D i v}}}_{D t}+\frac{D^{\star \star}}{f}\left(\frac{1}{f}{\overline{\left(\mathbf{u}^{\prime} q^{\prime}\right)_{R o t}}}\right)-\left(\frac{1}{f}{\overline{\left(\mathbf{u}^{\prime} q^{\prime}\right)_{R o t}}}\right) \cdot \nabla \overline{\mathbf{u}}^{\star}
$$

The zonal component of equation 5.31 is given by

$$
R H S=\bar{F}^{x}+{\overline{\left(v^{\prime} q^{\prime}\right)_{D i v}}}-\bar{u}^{\star} \partial_{x}\left(\frac{1}{f}{\overline{\left(u^{\prime} q^{\prime}\right)_{R o t}}}\right)-\left(\frac{1}{f}{\overline{\left(u^{\prime} q^{\prime}\right)_{R o t}}}\right) \partial_{x} \bar{u}^{\star} .
$$

We now scale each term the the above equation, as in chapter 2 :

$$
\begin{array}{cccc}
\bar{F}^{x} & {\overline{\left(v^{\prime} q^{\prime}\right)_{D i v}}} & \bar{u}^{\star} \partial_{x}\left(\frac{1}{f}{\overline{\left(u^{\prime} q^{\prime}\right)_{R o t}}}_{\text {R }}\right) & \left(\frac{1}{f}{\overline{\left(u^{\prime} q^{\prime}\right)}}_{R o t}\right) \partial_{x} \bar{u}^{\star} \\
\downarrow & \downarrow & \downarrow & \downarrow \\
F & \frac{U^{2}}{L} & \frac{U^{3}}{f L^{2}} & \frac{U^{3}}{f L^{2}} \\
\frac{F}{f U} & \frac{U}{f L} & \left(\frac{U}{f L}\right)^{2} & \left(\frac{U}{f L}\right)^{2}
\end{array}
$$

The last two terms are $O\left(R_{o}^{2}\right)$. It is consistent with the scaling in chapter 2 to neglect these terms since the Rossby number $R o \ll 1$. When the same scaling procedure is applied to the meridional component of equation 5.31, we find that equation 5.29 takes the simple form:

$$
\frac{D^{\star \star} \overline{\mathbf{u}}^{\star \star}}{D t}+f \mathbf{k} \times \overline{\mathbf{u}}^{\star \star}+\frac{1}{\rho_{0}} \nabla \bar{p}=\overline{\mathbf{F}}-\mathbf{k} \times{\overline{\left(\mathbf{u}^{\prime} q^{\prime}\right)}}_{D i v} .
$$


Replacing the $\overline{\mathbf{u}}^{\star}$ by $\overline{\mathbf{u}}^{\star \star}$ in the mean temperature equation (equation 2.55.a.b) and performing the scaling yields:

$$
\frac{D^{\star \star} \bar{T}}{D t}=\bar{G}
$$

Thus, the eddy term appears as a body force through the divergent component of the eddy flux of PV. This flux, as discussed in the beginning of this section, can be parameterized through a flux-gradient relationship in the form of equation 5.20. Thus the closure approach of chapter 3 is appropriate if viewed from the redefined TEM framework in which the eddy terms are represented by one term: the divergent eddy flux of PV.

The procedure would be to diagnose the quasigeostrophic potential vorticity and then determine the transfer coefficients that satisfy the appropriate momentum constraint for time mean flows. With knowledge of the parameterized PV flux, the governing equations (5.33 and 5.34) are closed, enabling determination of the mean velocity and temperature fields. Forcing by the divergent eddy flux of PV yields the mean velocity field $\overline{\mathbf{u}}^{\star \star}$, that naturally incorporates the momentum and temperature transferring properties of the quasigeostrophic eddy field.

There is however, a difficulty associated with equations 5.33 and 5.34 that must be noted. This difficulty is associated with the fact that the normal component of $\overline{\mathbf{u}}^{\star \star}$ does not necessarily vanish at the vertical boundaries. If the boundary conditions on the velocity are those of free slip, then $\bar{u}^{\star} \neq 0$ on boundaries that run north-south, and $\bar{v}^{\star} \neq 0$ on east-west boundaries, as discussed in section 2.6. The residual mean velocity does vanish, however, for no-slip boundary conditions. However, there is no a-priori reason why the term $\frac{1}{f} \mathbf{k} \times \nabla(\Lambda e)$ in equation 5.27 is zero at the boundaries. In fact, as figure 5.10 shows, the eddy enstrophy and its gradients were not zero along the western boundary and as a result $\overline{\mathbf{u}}^{\star \star}$ does not vanish there. This problematic feature must be addressed before implementation of the approach can proceed. 


\subsection{Summary}

The theoretical arguments and the eddy PV closure was tested using the three-dimensional, eddy-resolving, HPE model for a double-gyre ocean experiment. The eddy transfer characteristics of the flow were examined in the light of the time mean dynamical framework and subsequent closure for the eddy PV flux.

In the PV sheet, embedded in the upper level of the model, the time mean PV flux has a swirl-like structure, resulting in eddy fluxes of PV that were directed up as, well as down, the mean PV gradient.

However, the mean PV flux was shown to have a large nondivergent (rotational) component that had no dynamical effect in forcing the mean flow. The most problematic aspect of the chapter was in evaluating the divergent eddy PV fluxes. With the complicated boundary conditions, using a Poisson equation to determine the rotational flux would have been difficult indeed. Instead, the rotational and divergent fluxes were determined analytically using ideas advocated by Marshall and Shutts (1981). The divergent flux referred to in the chapter is not guaranteed to be purely divergent, since the method only gives the residual between the full PV flux and the rotational (nondivergent) component. It was assumed that mean PV contours were nearly parallel to mean streamlines, with $d \bar{\psi} / d \bar{q}$ and eddy enstrophy used to evaluate the rotational, and therefore, the divergent eddy PV flux.

The divergent eddy PV flux in the eddy intense jet region was found to be almost universally directed down the mean PV gradient. Much of the swirl structure present in the full PV flux was accounted for by the rotational component of the flux. Regions did exist where this flux was oriented upgradient. However, the along gradient component of this flux was vanishingly small when compared to the downgradient transfer regions.

This fact led us to propose couching the eddy flux term as the divergent eddy flux of PV, since a downgradient PV closure scheme seems justified for this flux component. The resultant governing equations are stated in equations 5.33 and 5.34 . 


\section{Chapter 6}

\section{Summary and Conclusions}

\subsection{Summary of the thesis}

In this thesis we have sought to improve the parametric representation of the transfer properties, and forcing of the mean flow by eddies, in non-eddy-resolving ocean models.

The advantage of the approach used here over conventional approaches, is that it avoids separating the heat and momentum (vorticity) transporting properties of the eddies and thus leads to a considerable simplification of the parameterization problem. It is also a more physically based approach than those currently used in ocean modeling. This thesis presents the method of parameterization and explores the scheme in light of resolved and parameterized eddy physics.

I would like to emphasize that the thesis does not proffer a recipe for eddy representation that will always work - the problem at its deepest level does not have a solution. Rather, a method is offered, that in one limit correctly captures quasigeostrophic eddy temperature and momentum transfer, and in another limit reduces to that of Gent and McWilliams (1990), which neglects momentum transfer.

The dynamical framework used is that of the "transformed Eulerian mean", first introduced by Andrews and McIntyre (1976). This gives a more complete picture of the propagation of the eddies, their transport, and their effects on the mean flow. 
Unfortunately, there is considerable confusion in the oceanographic community with regard to TEM, partly because of the success of the Gent and McWilliams (1990) scheme, and partly because GM was presented in a vacuum - without any reference to studies of the eddy problem in the atmosphere. Here, the TEM approach is correctly applied to the ocean, by including both momentum and temperature fluxes.

Chapter 2 introduces the TEM approach and presents the systematic scaling of the primitive equations for eddies that are assumed to be quasigeostrophic. This results in TEM sets for both zonal mean and time mean flows.

The governing TEM sets have two advantageous features; (1) Eddy disturbances are represented by one term only - an eddy PV flux. (2) The prognostic velocity is that of the "residual mean circulation", which under certain assumptions is equal to an "effective transport velocity" (Plumb and Mahlman (1987)). This is the relevant velocity for understanding meridional atmospheric tracer transport.

The eddy PV flux term appears as an effective body force in the mean momentum equations. For zonal flows, this PV flux is identically equal to the divergence of the Eliassen-Palm flux. With this interpretation of the body force, the propagation of eddy wave activity and the effect of the eddies on the mean flow is transparent. Equating the time mean eddy flux with the divergence of another flux yields a similar understanding for transient eddies in time mean flows (Plumb (1986)).

The Eliassen-Palm flux proves to be powerful when incorporating the role of boundaries, particularly when used in conjunction with the PV sheets introduced in section 2.2.2. This is because PV sheets are regions of concentrated Eliassen-Palm flux divergence representing PV fluxes associated with boundary temperature distributions.

When Reynolds stresses (eddy momentum fluxes) are neglected in the TEM approach for zonal flows, the scheme reduces to that advocated by Gent and McWilliams (1990), although the implementation is different. The analogy is not so exact for the time mean flows because the transformed time mean velocities in chapter 2 are different from those used by Gent and McWilliams. Their parameterization scheme has led to improvements 
in water mass distributions and transport, because they transform the density equation such that the eddy density flux terms are absent. Hence they are successful in combating the overly diffusive nature of numerical ocean models. However, failure to transform the momentum equations leads to vorticity being arbitrarily transferred down the mean gradient. By using the complete TEM framework here, we are able to encapsulate both the heat and momentum transporting properties of the eddy field without having to parameterize them separately.

When the eddy PV flux is parameterized, it is represented through a flux-gradient relationship. Thus, the parameterized eddies act to transfer PV down its mean gradient with transfer coefficients $K$. For zonal mean flows, the $K$ 's are chosen to satisfy an integral constraint on the eddy flux to ensure that the eddies act only to redistribute momentum in the flow. A parameterized model for time-mean flows is not presented, so we circumvent the difficult problem of having to establish momentum constraints for the time mean eddy flux of PV.

The downgradient closure for the PV flux does not necessarily mean that momentum is transferred downgradient (e.g. $\overline{u^{\prime} v^{\prime}} \neq-k \bar{u}_{y}$ ). As argued by Green (1970) and Marshall (1981), the pressure gradient forces act to ensure that the momentum of a fluid parcel is not conserved upon an eddy displacement. Hence, flux-gradient momentum transfer by the eddies is not appropriate.

In chapter 3 , the theoretical arguments and eddy PV closure were tested by comparing a three-dimensional, eddy-resolving, HPE model to a parameterized model. To my knowledge, this is the first time that the TEM set of equations have been made us of in a prognostic manner. Previous studies in the atmosphere that used the transformed Eulerian mean have all been diagnostic in nature. The experiments in this chapter focus on zonal flows because it is the simplest context in which to proceed. Here, eddy closure, although problematic, is at its most transparent.

For experiments which simulated stress-driven flow in a $\beta$-plane channel, mean fields and eddy transfer statistics were compared between the eddy-resolving and the param- 
eterized models. The comparison shows that the transformed Eulerian mean approach offers advantages over existing parameterization schemes. The parameterized model zonal mean fields closely matched those of the eddy resolving calculation in the equilibrated state. The close resemblance was achieved by correctly representing the transfer characteristics of the eddies. The eddy-resolved eddy-forcing term consisted of boundary sheets of eddy PV flux with small fluxes in the interior. The depth-integrated eddy-forcing of the mean zonal flow showed that the eddies acted to transfer momentum upgradient, thus strengthening and sharpening the jet. This is the first time, to my knowledge, that up-gradient momentum transfer has been successfully represented in a primitive equation ocean model.

The structure and size of this PV flux and its depth-integrated character was reproduced in the parameterized model. The meridional circulation in the parameterized model vanished so that there was no advection of temperature by the residual mean circulation. This agreed well with the diagnosed residual mean circulation in the eddyresolving calculation in which the stress-driven Eulerian mean circulation is balanced by the circulation associated with the eddy temperature fluxes.

Calculations in two more flow configurations further highlighted the advantage of our representation of quasigeostrophic eddies through a PV flux. The first was another comparison between eddy-resolving and the parameterized models. It concerned the spindown of a baroclinic zone. The sloping baroclinic zone spins down due to temperature transfer through finite-amplitude baroclinic instability which releases the available potential energy in the flow. However, after a finite amount of time, a cessation of mean flow energy release occurs due to the stabilizing effect of the planetary vorticity gradient. This stabilization evident in the eddy-resolving model is successfully reproduced in the parameterized model. Implementation of the Gent and McWilliams parameterization scheme, which is not based on PV transfer and PV gradients, results in a completely spun-down temperature field with no zonal mean flow. Thus, the physics of $\beta$-stabilization is absent from the Gent and McWilliams approach. 
In the second set of calculations, the parameterized model was applied to the atmosphere. It focused on the eddy forcing of the midlatitude tropospheric jets. The TEM approach gave realistic latitudes for the cores of the jets, and a realistic structure of the low-level winds when compared to observations. The Gent and McWilliams scheme was shown to be only a marginal improvement over not incorporating the eddies at all.

In recognition that the majority of ocean flows of interest cannot be modeled as zonal mean flows, chapter 5 explored the time mean TEM set presented in chapter 2. The objective was to understand the nature of the closure for the time mean eddy PV flux. The HPE model was employed to study the eddy transfer statistics in a double-gyre ocean. Diagnosed PV fluxes showed swirl patterns with fluxes that were directed up, as well as down, the mean PV gradient. Thus the flux-gradient relationship was invalidated for the time mean PV transfer. This arises through advection of eddy enstrophy by the mean flow, which gives rise to a nonlocal contribution to the eddy fluxes and results in upgradient eddy transfer. In spite of this, as Marshall and Shutts (1981) show, if the eddy statistics are quasigeostrophic then the eddy PV flux can be separated into two parts; a rotational, non-divergent flux and an irrotational, divergent flux. The rotational component balances the mean flow advection of the eddy enstrophy and is associated with the spatial growth and decay of the eddies. They are dynamically inert since they do not force the mean flow. The divergent flux balances the enstrophy dissipation, and as the diagnosed model results show, is directed down the mean PV gradient in the eddy intense jet region.

Thus a scheme is proposed in which the eddies are represented by a divergent eddy flux of PV with closure for this term focusing on downgradient PV transfer. The governing equations in which the only term representing the eddies appears as divergent eddy flux of PV are devised and stated.

This work, although addressing a difficult problem, has yielded the simple result that the correct way to parameterize quasigeostrophic eddies in numerical ocean simulations is through quasigeostrophic potential vorticity transfer. Of course, other parameterizations 
will be tried, but the TEM formulation and in the eddy flux of PV presented in this thesis is the natural approach to follow.

\subsection{Future Work}

The most tempting and the most obvious extension of this thesis work would be to implement the time mean approach in a parameterized model of three-dimensional flow. The first attempt should be in the double-gyre configuration used in the eddy resolving study of chapter 5 . I believe that it should be of a qualitative nature to determine if both the large scale mean flow patterns and regions of eddy forcing of the mean flow are reproduced. In effect, it would be the direct test of the arguments put forward in section 5.3. If, as the eddy-resolving three-dimensional calculation suggests, the reasoning behind the downgradient PV transfer representation is valid, then a detailed comparison between eddy resolving and parameterized models can be made.

In order to perform the above calculations, much thought and consideration will have to be given to the subtlety of the non-vanishing normal component at vertical boundaries, of the redefined residual mean velocity defined in equation 5.27. This normal flux would act to render non-conservation of mass, if the normal fluxes are not compensatory, and therefore tremendous numerical difficulties. It may be that this normal velocity is vanishingly small in some dynamical sense and can be justifiably set to zero with appropriate scaling arguments. Alternatively, the ad-hoc condition that this normal velocity vanishes could be applied and investigated. It is of course, much more appealing if the former is the case, and if so, would yield, through equations 5.33 and 5.34, a compelling, physically based method in which to represent eddies in ocean circulation models.

To complete the closure, the size and distribution of the transfer coefficients (the K's) need to be specified. The magnitude of the K's in any particular region should mirror the eddy activity there. The K's should be large in the vicinity of intense jet regions with significant enstrophy cascade and relatively small in the quiescent gyre interiors. In 
regions where the gradients of $\mathrm{PV}$ vanish, the choice of $\mathrm{K}$ is not all that critical because the parameterized flux will vanish anyway, due to the $K^{\prime} s$ acting on a zero $\nabla \bar{q}$ (as shown in chapter 4).

The opening sentence of this thesis states: "This thesis addresses the challenge of adequately representing the transfer properties, and the forcing of the mean flow, by unresolved eddy processes in numerical ocean simulations". Fundamentally, the representation problem does not have a solution. However, the method offered in the thesis does go a long way to offer an approach that adequately incorporates unresolved eddy transfer properties and their effects on the mean flow, and so provides a powerful conceptual framework for representing eddies in ocean circulation models. 


\section{Appendix A}

\section{Model details}

The numerical model used in this study is the MIT atmosphere-ocean hydrostatic, primitive equation (HPE) model described by Marshall (1997a,b). The code is written in Fortran and designed to exploit contemporary parallel processing computers. The design allows array processing features to map onto parallel architecture, permitting large intergrations to be carried out in a relatively short period of time when compared to serial processor machines.

\section{A.1 Model equations}

The equations used in the model code are written in terms of a generic vertical coordinate $r$. They have the same form as the conventional oceanic HPE's in z-coordinates which are isomorphic with the atmospheric HPE's in p-coordinates, so the code can be used to solve either equation set. Hence the model code has the working title of "MITgcmUV" where UV is the acronym for "ultra-versatile".

$$
\begin{aligned}
D_{t} \mathbf{v}+f \mathbf{k} \times \mathbf{v}+\frac{1}{\rho_{o}} \nabla_{r} \phi^{\prime} & =\mathcal{F}, \\
\epsilon_{n h} D_{t} \dot{r}-b^{\prime}+\frac{1}{\rho_{o}} \partial_{r} \phi^{\prime} & =\epsilon_{n h} \mathcal{F}_{\dot{r}}, \\
\nabla_{r} \cdot \mathbf{v}+\partial_{r} \dot{r} & =0,
\end{aligned}
$$




$$
\begin{aligned}
b^{\prime} & =b^{\prime}(\theta, S, r), \\
D_{t} \theta & =\mathcal{Q}_{\theta} \\
D_{t} S & =\mathcal{Q}_{s}
\end{aligned}
$$

where $\mathbf{v}=(u, v, 0)$ is the horizontal components of velocity, $\phi=g z$ is the geopotential, $b$ is the buoynacy, $\theta$ is the potential temperature, and $S$ is the salinity. $\mathcal{F}$ are sources/sinks of momentum, and $\mathcal{Q}_{\theta}$ and $\mathcal{Q}_{s}$ are sources/sinks of potential temperature and salinity respectively. $\epsilon_{n h}$ is a flag, which is set to 0 for hydrostatic calculations or 1 when the model is run non-hydrostatically.

In the ocean using z-coordinates:

$$
\begin{aligned}
r & =z \\
\dot{r} & =w \\
\rho_{o} & =\rho(20,35,0) \quad\left(\sim 1035 \mathrm{~kg} \mathrm{~m}^{-3} \text { using } \rho_{\text {eos } 80}\right) \\
b^{\prime}(\theta, S, r) & =\frac{-g}{\rho_{o}}\left(\rho(\theta, S, r)-\rho_{o}\right)
\end{aligned}
$$

produces the system of equations:

$$
\begin{aligned}
d_{t} \mathbf{v}+f \mathbf{k} \times \mathbf{v}+\frac{1}{\rho_{o}} \nabla_{z} p & =\mathcal{F} \\
\epsilon_{n h} d_{t} w+\frac{g \rho}{\rho_{o}}+\frac{1}{\rho_{o}} \partial_{z} p & =\epsilon_{n h} \mathcal{F}_{w} \\
\nabla_{z} \cdot \mathbf{v}+\partial_{z} w & =0 \\
\rho & =\rho(\theta, S), \\
d_{t} \theta & =\mathcal{Q}_{\theta}, \\
d_{t} S & =\mathcal{Q}_{s} .
\end{aligned}
$$

In the atmosphere using p-coordinates:

$$
\begin{aligned}
r & =p \\
\dot{r} & =\omega \\
\rho_{o} & =1
\end{aligned}
$$




$$
b^{\prime}(\theta, S, r)=-\frac{\alpha_{s}(r)}{\theta_{s}(r)} \theta^{\prime}
$$

produces a system of equations for the atmosphere

$$
\begin{aligned}
D_{t} \mathbf{v}+f \hat{\mathbf{k}} \wedge \mathbf{v}+\nabla_{p} \phi^{\prime} & =\mathcal{F} \\
\partial_{p} \phi^{\prime}+\alpha^{\prime} & =0 \\
\nabla_{p} \cdot \mathbf{v}+\partial_{p} \omega & =0 \\
\alpha^{\prime} & =\alpha_{o} \frac{\theta^{\prime}}{\theta_{o}} \\
D_{t} \theta^{\prime}+\omega \partial_{p} \theta_{o} & =\frac{\theta}{T} \frac{\mathcal{Q}}{c_{p}}
\end{aligned}
$$

where $\alpha=1 / \rho$ is the specific volume, and it has been convenient to split out a reference state $\left(\theta_{o}, \alpha_{o}\right.$ and $\phi_{o}$ in the following manner:

$$
\begin{aligned}
\theta & =\theta_{o}+\theta^{\prime} \\
\alpha & =\alpha_{o}+\alpha^{\prime} \\
\phi & =\phi_{o}+\phi^{\prime}
\end{aligned}
$$

where the reference state satisfies the equations of a resting state:

$$
\begin{aligned}
\theta_{o} & =\theta_{o}(p) \\
\alpha_{o} & =\frac{R}{p}\left(\frac{p}{p_{c}}\right)^{\kappa} \theta_{o} \\
\partial_{p} \phi_{o} & =-\alpha_{o}
\end{aligned}
$$

with the boundary condition that $\phi_{o}\left(p=p_{s}\right)=\phi_{s}(x, y)$.

\section{A.2 MITgcmUV solution procedure}

Equations A.1 can be expressed as a set of P.D.E's. These equations form the basis of the MIT atmosphere-ocean model.

In equations $\mathrm{A} 1$, the total pressure perturbation can be divided into three parts; a surface part, $\phi_{s u r f}^{\prime}(x, y)$, a hydrostatic part $\phi_{h y d}^{\prime}(x, y, r)$ and a non-hydrostatic part 
$\phi_{n h}^{\prime}(x, y, r)$ so that

$$
\phi^{\prime}(x, y, r)=\phi_{\text {surf }}^{\prime}(x, y)+\phi_{h y d}^{\prime}(x, y, r)+\epsilon_{n h} \phi_{n h}^{\prime}(x, y, r)
$$

Whether $\phi^{\prime}$ is pressure (ocean model) or geopotential (atmospheric model), the horizontal pressure gradient term becomes

$$
\frac{1}{\rho_{o}} \nabla_{r} \phi_{\text {surf }}^{\prime}=g \nabla_{r} \eta
$$

where $\eta$ is the free-surface height in units of $r$. Substituting A7 into equations A1 gives:

$$
\begin{aligned}
\partial_{t} \mathbf{v}+g \nabla_{r} \eta+\epsilon_{n h} \frac{1}{\rho_{o}} \nabla_{r} \phi_{n h}^{\prime} & =\mathbf{G}_{v} \\
\epsilon_{n h} \frac{\partial \dot{r}}{\partial t}+\epsilon_{n h} \frac{1}{\rho_{o}} \partial_{r} \phi_{n h}^{\prime} & =\epsilon_{n h} G_{\dot{r}} \\
\frac{1}{\rho_{o}} \partial_{r} \phi_{h y d}^{\prime} & =b^{\prime} \\
\nabla_{r} \cdot \mathbf{v}+\partial_{r} \dot{r} & =0 \\
b^{\prime} & =b^{\prime}(\theta, S, r) \\
\partial_{t} \theta & =G_{\theta} \\
\partial_{t} S & =G_{s}
\end{aligned}
$$

where

$$
\begin{aligned}
\mathbf{G}_{v} & =-\mathbf{v} \cdot \nabla_{r} \mathbf{v}-\frac{1}{\rho_{o}} \nabla_{r} \phi_{h y d}^{\prime}+\mathcal{F}_{v} \\
G_{\dot{r}} & =-\mathbf{v} \cdot \nabla_{r} \dot{r}+\mathcal{F}_{\dot{r}} \\
G_{\theta} & =-\mathbf{v} \cdot \nabla_{r} \theta+\mathcal{Q}_{\theta} \\
G_{S} & =-\mathbf{v} \cdot \nabla_{r} S+\mathcal{Q}_{S}
\end{aligned}
$$

Note that in the hydrostatic limit $\left(\epsilon_{n h}=0\right)$, equation A8b vanishes. The hydrostatic pressure is found by integrating A8c with the boundary condition that $\phi_{h y d}^{\prime}\left(r=r_{\text {surf }}\right)=$ 0 :

$$
\begin{aligned}
& \int_{r^{\prime}}^{r_{\text {surf }}} \partial_{r} \phi_{h y d}^{\prime} d r=\left[\phi_{h y d}^{\prime}\right]_{r^{\prime}}^{r_{\text {surf }}}=\int_{r^{\prime}}^{r_{\text {surf }}} \rho_{o} b^{\prime} d r \\
\Rightarrow \quad & \phi_{h y d}^{\prime}\left(x, y, r^{\prime}\right)=-\int_{r^{\prime}}^{r_{\text {surf }}} \rho_{o} b^{\prime} d r
\end{aligned}
$$


We discretize equations A8 in time as follows:

$$
\begin{aligned}
\mathbf{v}^{(n+1)}+\Delta t g \nabla_{r} \eta^{(n+1)}+\frac{\Delta t \epsilon_{n h}}{\rho_{o}} \nabla_{r} \phi_{n h}^{\prime}{ }^{(n+1)} & =\mathbf{v}^{*} \\
\epsilon_{n h}\left(\dot{r}^{(n+1)}+\frac{\Delta t}{\rho_{o}} \partial_{r} \phi_{n h}^{\prime}{ }^{(n+1)}\right) & =\epsilon_{n h} \dot{r}^{*} \\
\frac{1}{\rho_{o}} \partial_{r} \phi_{h y d}^{\prime}{ }^{(n)} & =b^{\prime(n)} \\
\nabla_{r} \cdot \mathbf{v}^{(n+1)}+\partial_{r} \dot{r}^{(n+1)} & =0 \\
b^{\prime(n)} & =b^{\prime}\left(\theta^{(n)}, S^{(n)}, r\right) \\
{\left[1-\partial_{r} \kappa_{\theta}^{r} \partial_{r}\right] \theta^{(n+1)} } & =\theta^{*} \\
{\left[1-\partial_{r} \kappa_{S}^{r} \partial_{r}\right] S^{(n+1)} } & =S^{*}
\end{aligned}
$$

where

$$
\begin{aligned}
v^{*} & =\mathbf{v}^{(n)}+\Delta t \mathbf{G}_{v}^{\left(n+\frac{1}{2}\right)} \\
\dot{r}^{*} & =\dot{r}^{(n)}+\Delta t G_{\dot{r}}^{\left(n+\frac{1}{2}\right)} \\
\theta^{*} & =\theta^{(n)}+\Delta t G_{\theta}^{\left(n+\frac{1}{2}\right)} \\
S^{*} & =S^{(n)}+\Delta t G_{S}^{\left(n+\frac{1}{2}\right)}
\end{aligned}
$$

An equation for $\eta$ is obtained by integrating the continuity equation over the entire depth of the fluid $(R(x, y))$, using Leibniz formula:

$$
\epsilon_{r l} \partial_{t} \eta=\left.\dot{r}\right|_{r=r_{s u r f}}=-\nabla_{r} \cdot \int_{R(x, y)}^{r_{s u r f}} \mathbf{v} d r
$$

Where $\epsilon_{r l}$ is a flag to distinguish between a free-surface equation $\left(\epsilon_{r l}=1\right)$ or the rigid-lid approximation $\left(\epsilon_{r l}=0\right)$. Discretizing in-time:

$$
\epsilon_{r l} \eta^{(n+1)}+\Delta t \nabla_{r} \cdot \int_{R(x, y)}^{r_{s u r f}} \mathbf{v}^{(n+1)} d r=\epsilon_{r l} \eta^{(n)}
$$

Substituting A9a into A10, assuming $\epsilon_{n h}=0$ yields a Helmholtz equation for $\eta^{(n+1)}$ :

$$
\epsilon_{r l} \eta^{(n+1)}-\nabla_{r} \cdot \Delta t^{2} g\left(r_{s u r f}-R(x, y)\right) \nabla_{r} \eta^{(n+1)}=\eta^{*}
$$


where

$$
\eta^{*}=\epsilon_{r l} \eta^{(n)}-\Delta t \nabla_{r} \cdot \int_{R(x, y)}^{r_{\text {surf }}} \mathbf{v}^{*} d r
$$

When the model is non-hydrostatic $\left(\epsilon_{n h}=1\right)$ we need an equation for $\phi_{n h}^{\prime}$. This is obtained by sustituting A9a and A9b into A9d:

$$
\left[\nabla_{r}^{2}+\partial_{r r}\right] \phi_{n h}^{\prime(n+1)}=\frac{\rho_{o}}{\Delta t}\left(\nabla_{r} \cdot \mathbf{v}^{* *}+\partial_{r} \dot{r}^{*}\right)
$$

where

$$
\mathbf{v}^{* *}=\mathbf{v}^{*}-\Delta \operatorname{tg} \nabla_{r} \eta^{(n+1)}
$$

Finally, the horizontal velocities at the new time level are found by:

$$
\mathbf{v}^{(n+1)}=\mathbf{v}^{* *}-\epsilon_{n h} \frac{\Delta}{\rho_{c} s t} \nabla_{r} \phi_{n h}^{\prime(n+1)}
$$

and the vertical velcity is found by integrating continuity vertically.

\section{A.3 Boundary conditions}

I make the rigid-lid approximation where the upper surface is held fixed and then interpret it to exert a pressure on the fluid.

\section{Upper and lower boundary conditions}

$\bar{q}$ and $\psi$

At the upper and lower boundaries there exists an established temperature distribution. equation (2.5) provides inhomogeneous Neumann boundary conditions on $\psi$ at the horizontal boundaries since $\partial \psi / \partial z$ is specified there. A computational and conceptual simplification can be made if we replace the the inhomogeneous Neumann boundary conditions by homogeneous ones, following Bretherton (1966). Let us define the quasigeostrophic potential vorticity $\tilde{q}(y, z)$, which is equal to $\bar{q}(y, z)$ in the fluid interior, except adjacent to the horizontal boundaries. Just inside these boundaries, we place delta-function sheets 
of PV,$\delta q_{\text {upper }}$ and $\delta q_{\text {lower }}$, with the size and shape of each representing the magnitude and structure of the temperature distribution on the boundary. Thus:

$$
\tilde{q}=\bar{q}+\delta q_{\text {upper }}+\delta q_{\text {lower }},
$$

where the delta-function sheets are given by

$$
\delta q_{\text {upper }}=\frac{f_{0}^{2}}{N^{2}} \frac{\partial \bar{\psi}}{\partial z} \quad ; \quad \delta q_{\text {lower }}=-\frac{f_{0}^{2}}{N^{2}} \frac{\partial \bar{\psi}}{\partial z}
$$

The temperature perturbations at the upper and lower boundaries are set to zero, with the actual temperature variation appearing in the PV distribution as delta-function sheets of PV just interior to the boundary.

$\underline{E}$ and $\bar{w}^{\star}$

The fact that we choose to employ homogeneous Neumann boundary conditions on $\psi$ (by setting the temperature perturbation to zero) dictates that the lateral temperature flux at the horizontal boundaries vanishes. Hence the component of the Eliassen-Palm flux $\left(E_{z}\right)$ through the boundary is zero. This gives us the momentum integral constraint (equation (2.71)):

$$
\int_{\text {Volume }} \underline{\nabla} \cdot \underline{E} d V=0 .
$$

Moreover, this provides the needed boundary condition on the vertical component, of the residual mean circulation at the upper and lower boundaries. Because the lateral temperature flux is zero on the boundary we have $\bar{w}^{\star}=0$. This is the same as that used by Gent and McWilliams (1990) and Gent et al. (1995) where they insist that the normal component of the 'eddy-induced' velocity at the boundary is zero. The upper and lower boundary conditions on $\bar{w}^{\star}$ are discussed in detail by Treguier et al. (1997) mainly because they consider difficulties which arise with small vertical stratification in the surface mixed layer. To avoid erroneously large $\bar{w}^{\star}$ where isopycnals are vertical, the transfer coefficient is set to zero at the horizontal boundaries. This is in complete contrast to the transfer coefficients of PV which necessarily have to be nonzero to obtain the flux contribution due to the PV sheets. 


\section{REFERENCES}

Andrews, D.G., 1990: On the forcing of time-mean flows by transient, small-amplitude eddies. J. Atmos. Sci., 47, 1837-1844.

Andrews, D.G., and M.E. McIntyre, 1976: Planetary waves in horizontal and vertical shear: the generalized Eliassen-Palm relation and the mean zonal acceleration. $J$. Atmos. Sci., 33, 2031-2048.

Andrews, D.G., and M.E. McIntyre, 1978a: Generalized Eliassen-Palm and CharneyDrazin theorems for waves on axisymmetric flows in compressible atmospheres. $J$. Atmos. Sci., 35, 175-185.

Andrews, D.G., and M.E. McIntyre, 1978b: An exact theory for nonlinear waves on a Lagrangian-mean flow. J. Fluid Mech., 89, 609-646.

Böning, C.W., W.R. Holland, F.O Bryan, G. Danabasoglu, and J.C. McWilliams, 1995: An overlooked problem in model simulations of the thermohaline circulation and heat transport in the Atlantic ocean. J. Climate, 8 515-523.

Bluestein, H.B., 1993: Synoptic-dynamic meteorology in midlatitudes. Volume II: Observations and theory of weather systems. Oxford University Press, New York, NY.

Bretherton, F., 1966: Critical layer instability in baroclinic flows. Quart. J. Roy. Meteor. Soc., 92, 325-334.

Bretherton, F.P., and M. Karweit, 1975: Mid-ocean mesoscale modeling. Numerical Models of Ocean Circulation, Nat. Acad. Sci, 237-249.

Bryden, H.L., 1979: Poleward heat flux and conversion of available potential energy in Drake Passage. J. Mar. Res., 37, 1-22.

Bryden, H.L., and E.C. Brady, 1989: Eddy momentum and heat fluxes and their effects on the circulation of the equatorial Pacific Ocean. J. Mar. Res., 47, 55-79.

Cessi, P.R., Condie, R.V., and Young, W.R., 1990: Dissipative dynamics of western boundary currents. J. Mar. Res., 48, 677-700.

Charney, J.G., 1947: The dynamics of long waves in a baroclinic westerly current. J. Meteorol., 4, 135-163.

Charney, J.G., 1955: The Gulf Stream as an inertial boundary layer. Proc. Natl. Acad. Sci. U.S.A. 41, 731-740. 
Charney, J.G., and P.G. Drazin, 1961: Propagation of planetary-scale disturbances from the lower into the upper atmosphere. J. Geophys. Res., 66, 83-109.

Charney, J.G., and M.E. Stern, 1962: On the stability of internal baroclinic jets in a rotating atmosphere. J. Atmos. Sci., 19, 159-172.

Cox, M.D., 1987: Isopycnal diffusion in a z-coordinate model. Ocean modeling, 74, 1-5.

Danabasoglu, G., and J.C. McWilliams, 1995: Sensitivity of the global ocean circulation to parameterizations of mesoscale tracer transports. J. Climate, 8, 2967-2978.

Dantzler, H.L., Jr. 1977: Potential energy maxima in the tropical and subtropical North Atlantic. J. Phys. Oceanogr. 7, 512-519.

Dukowicz, J.K., and R.J. Greatbatch, 1997: The bolus velocity in the stochastic theory of ocean turbulent tracer transport. Submitted to J. Phys. Oceanogr.

Eady, E.T., 1949: Long waves and cyclone waves. Tellus, 1, 33-52.

Edmon, H.J., Jr., B.J. Hoskins, and M.E. McIntyre, 1980: Eliassen-Palm cross sections for the troposphere. J. Atmos. Sci., 37, 2600-2616.

Eliassen, A., and E. Palm, 1961: On the transfer of energy in stationary mountain waves. Geofys. Publ., 22, No. 3, 1-23.

England, M., 1995: Using chlorofluorocarbons to assess ocean climate models. Geophys. Res. Lett., 22, 3021-3054.

Fofonoff, N.P., 1954: Steady flow in a frictionless homogeneous ocean. J. Mar. Res, 13, 254-262.

Gent, P.R., and J.C. McWilliams, 1990: Isopycnal mixing in ocean circulation models. J. Phys. Oceanogr., 20, 150-155.

Gent, P.R., J. Willebrand, T.J. McDougall and J.C. McWilliams, 1995: Parameterizing eddy-induced tracer transports in ocean circulation models. J. Phys. Oceanogr., $25,463-474$.

Gent, P.R., and J.C. McWilliams, 1996: Eliassen-Palm fluxes and the momentum equation in non-eddy resolving ocean circulation models. J. Phys. Oceanogr., 26, 2539-2546.

Gill, A.E., J.S.A. Green, and A. Simmons, 1974: Energy partition in the large-scale ocean circulation and the production of mid-ocean eddies. Deep-Sea Res., 21. 499528. 
Greatbach, R.J., 1988: On the scaling of inertial subgyres. Dyn. Atmos. and Oceans., 12, 265-285.

Greatbach, R.J., 1998: Exploring the relationship between eddy-induced transport velocity, vertical momentum transfer, and the isopycnal flux of potential vorticity. $J$. Phys. Oceanogr., 28, 422-432.

Greatbach, R.J., and K.G. Lamb, 1990: On parameterizing vertical mixing of momentum in non non-eddy-resolving models. J. Phys. Oceanogr., 20, 1634-1637.

Green, J., 1970: Transfer properties of the large-scale eddies and the general circulation of the atmosphere, Quart. J. Roy. Meteor. Soc., 96, 157-185.

Haynes, P.H., and M.E. McIntyre 1990: On the conservation and impermeability theorems for potential vorticity. J. Atmos. Sci., 47, $2021-2031$.

Held I.M., and A. Hou, 1980: Nonlinear axially symmetric circulations in a nearly inviscid atmosphere. J. Atmos. Sci., 37, 515-533.

Held, I.M., and M.J. Suarez, 1994: A proposal for the intercomparison of the dynamical cores of atmospheric general circulation models. Bull. Amer. Meteor. Soc., 75, No. 10, 1825-1830.

Hirst, A.C., and T.J. McDougall, 1996: Deep-water properties and surface buoyancy flux as simulated by a $\mathrm{z}$-coordinate model including eddy-induced advection. $J$. Phys. Oceanogr., 26, 1320-1343.

Hogg, N.G., 1993: Toward parameterization of the eddy field near the Gulf Stream. Deep-Sea Res., 40, 2359-2376.

Holland, W.R., 1978: The role of mesoscale eddies in the general circulation. J. Phys. Oceanogr., 8, 363-392.

Holland, W.R., and L.B. Lin, 1975: On the generation of mesoscale eddies and their contribution to the oceanic general circulation. J. Phys. Oceanogr., 5, 642-669.

Holland, W.R., and P.B. Rhines, 1979: An example of eddy-induced ocean circulation. J. Phys. Oceanogr., 9, 1010-1031.

Holland, W.R., and W.J. Schmitz Jr., 1985: Zonal penetration scale of model midlatitude jets. J. Phys. Oceanogr., 15, 1859-1875.

Holopainen, E., 1984: Statistical local effect of synoptic-scale transient eddies on the time-mean flow in the northern extratropics in winter. J. Atmos. Sci., 41, 25052515. 
Hoskins, B.J., 1983: Modeling the transient eddies and their feedback on the mean flow. Large-Scale Dynamical Processes in the Atmosphere, B.J. Hoskins, R.P. Pearce, Eds., Academic.

Hoskins, B.J., I.N. James, and G.H White, 1983: Shape, propagation, and mean-flow interaction of large-scale weather systems. J. Atmos. Sci., 40, 1595-1612.

Hoskins, B.J., M.E. McIntyre, and A.W. Robertson, 1985: On the use and significance of isentropic potential vorticity maps. Quart. J. Roy. Meteor. Soc., 111, 877-946.

Houghton, J.T., 1977: The Physics of Atmospheres. Cambridge University Press.

Illari, L., and Marshall, J., 1983: On the interpretation of eddy fluxes during a blocking episode. J. Atmos. Sci., 40, 2232-2242.

Jeffreys H., 1926: On the dynamics of geostrophic winds. Quart. J. Roy. Meteor. Soc., $52,85-102$.

Johnson, G.C., and H.L. Bryden, 1989: On the size of the Antarctic Circumpolar Current. Deep-Sea Res., 36, 39-53.

Killworth, P.D., 1997: On the parameterization of eddy transfer, Part I: Theory. $J$. Mar. Res., 55, 1171-1197.

Killworth, P.D., 1998: On the parameterization of eddy transfer, Part II: Tests with a channel model. J. Mar. Res., 56, 349-374.

Lee, M,-M, and H. Leach, 1996: Eliassen-Palm flux and eddy potential vorticity flux for a nonquasigeostrophic time-mean flow. J. Phys. Oceanogr., 26, 1304-1319.

Lee, M,-M, D.P. Marshall, and R.G. Williams, 1997: On the eddy transfer of tracers: advective or diffusive? J. Mar. Res., 55, 1-24.

Lorenz, E.N., 1967: The nature and theory of the general circulation of the atmosphere. WMO publication, 218, World Meteorological Organization, Geneva, Switzerland, $161 \mathrm{pp}$.

Lorenz, E.N., 1968: Climate determinism. Meteorological monographs, 8, 1-3.

Marshall, D., and J. Marshall, 1992: Zonal penetration scale of midlatitude oceanic jets. J. Phys. Oceanogr., 22, 1018-1032.

Marshall, D., R.G. Williams, and M,-M, Lee, 1998: On the relation between eddyinduced transport and isopycnic gradients of potential vorticity. Submitted to $J$. Phys. Oceanogr. 
Marshall, J., 1981:. On the parameterization of geostrophic eddies in the ocean J. Phys. Oceanogr., 11, 257-271.

Marshall, J., 1984: Eddy-mean-flow interaction in a barotropic ocean model. Quart. J. Roy. Meteor. Soc., 101, 573-590.

Marshall, J., and G. Shutts, 1981. A note on rotational and divergent eddy fluxes. J. Phys. Oceanogr., 11, 1677-1680.

Marshall, J., D. Olbers, H. Ross, and D. Wolf-Gladrow, 1993: Potential vorticity constraints on the dynamics and hydrography of the Southern Ocean. J. Phys. Oceanogr., 23, 465-487.

Marshall, J., C. Hill, L. Perelman, and A. Adcroft, 1997a: Hydrostatic, quasi-hydrostatic, and nonhydrostatic ocean modeling. J. Geophys. Res., 102, No. C3, 5733-5752.

Marshall, J., A. Adcroft, C. Hill, L. Perelman, and C. Heisey, 1997b: A finite volume, incompressible Navier Stokes model for studies of the ocean on parallel computers. J. Geophys. Res., 102, No. C3, 5753-5766.

McDougall, T.J., and P.C. McIntosh, 1996a: The temporal-residual mean velocity. Part I: Derivation and the scalar conservation equations. J. Phys. Oceanogr., 26, 26532665.

McDougall, T.J., and P.C. McIntosh, 1996b: The temporal-residual mean velocity. Fart II: The isopycnal interpretation of the TRM velocity and scalar conservation equations. submitted to J. Phys. Oceanogr.

McIntosh, P.C., and T.J. McDougall, 1996: Isopycnal averaging and the residual mean circulation. J. Phys. Oceanogr., 26, 1655-1660.

McIntyre, M.E., 1980: Introduction to the generalized Lagrangian-mean description of wave-mean flow interaction. Pure Appl. Geophys., 118, 152-176.

McWilliams, J.C., 1983: On the mean dynamical balances of the Gulf Stream recirculation zone. J. Mar. Res., 41, 427-460.

McWilliams, J.C., W.R. Holland and J.H.S. Chow, 1978: A description of numerical Antarctic Circumpolar currents. Dyn. Atmos. Oceans, 2, 213-291.

McWilliams, J.C., and P.R. Gent, 1994: The wind-driven ocean circulation with an isopycnal-thickness mixing parameterization. J. Phys. Oceanogr., 24, 46-65.

Morgan, G.W., 1956: On the wind-driven ocean circulation. Tellus, 8, 301-320.

Munk, W.H., 1950: On the wind-driven ocean circulation. J. Meteorol., 7, 79-93. 
Palmer, T.N., 1981: Diagnostic study of a wavenumber-2 stratospheric sudden warming in a transformed Eulerian-mean formalism. J. Atmos. Sci., 38, 844-855.

Pavan, V., and I.M. Held, 1996: The diffusive approximation for eddy fluxes in baroclinically unstable jets. J. Atmos. Sci., 53, 1262-1272.

Pedlosky, J., 1975: A note on the amplitude of baroclinic waves in the mid-ocean. Deep-Sea Res., 22, 575-576.

Pedlosky, J., 1977: On the radiation of mesoscale energy in the mid-ocean. Deep-Sea Res., 24, 591-600.

Pedlosky, J., 1987: Geophysical Fluid Dynamics, Springer-Verlag, New York, second edition.

Pedlosky, J., 1996: Ocean Circulation Theory. Springer-Verlag, New York.

Pierrehumbert, R.T., and K.L. Swanson, 1995: Baroclinic instability. Ann. Rev. Fluid. Mech., 27, 419-467.

Philander, S.G.H., 1978: Forced oceanic waves. Rev. Geophys. Res., 16, 15-46.

Plumb, R.A., 1986: Three-dimensional propagation of transient quasi-geostrophic eddies and its relationship with the eddy forcing of the time-mean flow. J. Atmos. Sci., $43,1657-1678$.

Plumb, R.A., 1990: A nonacceleration theorem for transient quasi-geostrophic eddies on a three-dimensional time-mean flow. J. Atmos. Sci., 47, 1825-1836.

Plumb, R.A., and J. Mahlman, 1987: The zonally averaged transport characteristics of the GFDL general circulation/transport model. J. Atmos. Sci., 44, 298-327.

Redi, M.H., 1982: Oceanic isopycnal mixing by coordinate rotation. J. Phys. Oceanogr., 12, 1154-1158.

Rhines, P.B., 1977: The dynamics of unsteady currents. The Sea, Vol. 6, E. Goldberg, Ed., 189-318.

Rhines, P.B., 1986: Vorticity dynamics of the oceanic general circulation. Annu., Rev. Fluid Mech., 18, 433-497.

Rhines, P.B., and W.B. Holland, 1979: Theoretical discussion of eddy-driven mean flows. Dyn. Atmos. and Oceans. 3, 289-325.

Rhines, P.B., and W.R. Young, 1982: Homogenization of potential vorticity in planetary gyres. J. Fluid Mech., 122, 347-367. 
Richardson, P.L., 1983: Eddy Kinetic energy in the North Atlantic from surface drifters. J. Geophys. Res., 88(C7), 4355-4367.

Richman, J.G., C. Wunsch, and N.G. Hogg, 1977: Space and time scales and mesoscale motions in the sea. Rev. Geophys., 15, 385-420.

Robinson, A.G., and J.C. McWilliams, 1974: The baroclinic instability of the open ocean. J. Phys. Oceanogr., 4, 281-294.

Robitaille, D.V., and A.J. Weaver, 1995: Validation of sub-grid scale mixing schemes using CFC's in a global ocean model. Geophys. Res. Lett., 22, 2917-2920.

Schmitz, William J., Jr., 1978: Observations of the vertical distribution of low frequency kinetic energy in the western North Atlantic. J. Mar. Res., 36, 295-310.

Schmitz, W.J., Jr., and J.R. Luyten, 1991: Spectral time scales for mid-latitude eddies. J Mar. Res., 49, 75-105.

Schubert, S., C.K. Park, W. Higgins, S. Moorthi, and M. Suarez, 1990: An Atlas of ECMWF Analyses (1980-1987) Part I - First Moment Quantities. NASA Technical Memorandum 100747.

Semtner, A.J.,Jr., and R.M. Chervin, 1992: Ocean general circulation from a global eddy-resolving model. J. Geophys. Res., 97, No. C4, 5493-5550.

Simmons, A.J., 1974: The meridional scale of baroclinic waves. J. Atmos. Sci., 31, $1515-1525$.

Stammer, D. 1997: Global characteristics of ocean variability estimated from regional TOPEX/POSEIDON altimeter measurements. J. Phys. Oceanogr., 27, 1743-1769.

Stammer, D., and C. Wunsch, 1994: Preliminary assessment of the accuracy and precision of TOPEX/POSEIDON altimeter data with respect to the large-scale ocean circulation. J. Geophys. Res., 99, 24584-24604.

Starr, V.P., 1948: An essay on the general circulation of the earth's atmosphere. $J$. Meteor., 5, 39-43.

Starr, V.P., 1968: The Physics of Negative Viscosity Phenomena. McGraww-Hill.

Stone, P., 1972: A simplified radiative-dynamical model for the static stability of rotating atmospheres. J. Atmos. Sci., 29, 405-418.

Stull, R.B., 1988: An Introduction to Boundary Layer Meteorology. Kluwer Academic Publishers. 
Sverdrup, H.U., 1947: Wind-driven ocean currents in a baroclinic ocean, with application to the equatorial currents of the eastern Pacific. Proc. Natl. Acad. Sci. U.S.A. 33, 318-326.

Tandon, A., and C. Garret, 1996: On a recent parameterization of mesoscale eddies. J. Phys. Oceanogr., 26, 406-411.

Tennekes, H., and J.L. Lumley, 1972: A First Course in Turbulence. The MIT Press.

Treguier, A.M., I.M. Held, and V.D. Larichev, 1997: Parameterization of quasigeostrophic eddies in primitive equation ocean models. J. Phys. Oceanogr., 27, 567-580.

Trenberth, K.E., 1986: Assessment of the impact of transient eddies on the zonal flow during a blocking episode using localized Eliassen-Palm flux diagnostics. J. Atmos. Sci., 43, 2070-2087.

Veronis, G., 1966: Wind-driven ocean circulation Part II. Deep-Sea Res., 13, 30-55.

Visbeck, M., J. Marshall, T. Haine and M. Spall, 1997: On the specification of eddy transfer coefficients in coarse resolution ocean circulation models. J. Phys. Oceanogr., 27, 381-402.

Webster, F., 1965: Measurements of eddy fluxes of momentum in the surface layer of the Gulf Stream. Tellus, 17, 239-245.

Welander, P., 1973: Lateral friction in the ocean as an effect of potential vorticity mixing. Geophys. Fluid Dyn., 5, 101-120.

Wunsch, C., 1981: Low-frequency variability of the sea: Evolution of Physical Oceanography, B.A. Warren, and C. Wunsch, Eds., The MIT Press.

Wunsch, C., and D. Stammer, 1995: The global frequency-wavenumber spectrum of oceanic variability estimated from TOPEX/POSEIDON altimeter measurements. J. Geophys. Res., 100, 24895-24910.

Wyrtki, K., L. Magaard, and J. Hager, 1976: Eddy energy in the oceans. J. Geophys. Res., 81, 2641-2646. 